This item was submitted to Loughborough's Research Repository by the author.

Items in Figshare are protected by copyright, with all rights reserved, unless otherwise indicated.

\title{
Stitched transmission lines for wearable RF devices
}

PLEASE CITE THE PUBLISHED VERSION

PUBLISHER

(C) Isaac Hyuk Daniel

\section{PUBLISHER STATEMENT}

This work is made available according to the conditions of the Creative Commons Attribution-NonCommercialNoDerivatives 4.0 International (CC BY-NC-ND 4.0) licence. Full details of this licence are available at: https://creativecommons.org/licenses/by-nc-nd/4.0/

\section{LICENCE}

CC BY-NC-ND 4.0

\section{REPOSITORY RECORD}

Daniel, Isaac H.. 2019. "Stitched Transmission Lines for Wearable RF Devices". figshare. https://hdl.handle.net/2134/25581. 


\section{STITCHED TRANSMISSION LINES FOR WEARABLE RF DEVICES}

By

Isaac Hyuk Daniel

A Doctoral Thesis Submitted in Partial Fulfilment of the Requirements for the Award of Doctor of Philosophy of Loughborough University

September 2016

(C) by Isaac Hyuk Daniel 


\section{Abstract}

With the rapid growth and use of wearable devices over the last decade, the advantages of using portable wearable devices are now been utilized for day to day activities. These wearable devices are designed to be flexible, low-profile, light-weight and smoothly integrated into daily life. Wearable transmission lines are required to transport RF signals between various pieces of wearable communication equipment and to connect fabric based antennas to transmitters and receivers; the stitched transmission line is one of the hardware solutions developed to enhance the connectivity between these wearable devices. Textile manufacturing techniques that employ the use of sewing machines alongside conductive textile materials can be used to fabricate the stitched transmission line.

In this thesis, the feasibility of using a sewing machine in the fabrication of a novel stitched transmission line for wearable devices using the idea of a braided coaxial cable have been examined. The sewing machine used is capable of a zig-zag stitch with approximate width and length within the range of $0-6 \mathrm{~mm}$ and $0-4 \mathrm{~mm}$ respectively. The inner conductor and the tubular insulating layer of the stitched transmission lines were selected as RG 174, while the stitched shields were made up of copper wires and conductive threads from Light Stitches®. For shielding purpose, the structure is stitched onto a denim material with a conductive thread with the aid of a novel manufacturing technique using a standard hardware. The Scattering Parameters of the stitched transmission line were investigated with three different stitch angles $85^{\circ}, 65^{\circ}$ and $31^{\circ}$ through simulation and experiments, with the results demonstrating that the stitched transmission line can work usefully and consistently from 0.04 to $4 \mathrm{GHz}$. The extracted Scattering parameters indicated a decrease in DC loss with increased stitch angle and an increase in radiation loses, which tends to increase with increase in frequency. The proposed stitched transmission line makes a viable transmission line but a short stitch length is associated with larger losses through resistance. The DC losses observed are mainly influenced by the resistance of the conductive threads at lower frequencies while the radiation losses are influenced by the wider apertures related to the stitch angles and increase in frequency along the line.

The performances of the stitched transmission line with different stitch patterns, when subjected to washing cycles and when bent through curved angles $90^{\circ}$ and $180^{\circ}$ were also investigated and results presented. 
Also, the sensitivity of the design to manufacturing tolerances was also considered. First, the behavior of the stitched transmission line with two different substrates Denim and Felt were investigated with the results indicating an insignificant increase in losses with the Denim material. Secondly, the sensitivity of the design with variations in cross section dimensions was investigated using numerical modeling techniques and the results showed that the impedance of the stitched transmission line increases when the cross-sectional dimensions are decreased by $0.40 \mathrm{~mm}$ and decreases when the cross sectional dimensions are increased by $0.40 \mathrm{~mm}$. Equally, repeatability of the stitched transmission line with three different stitch angles $85^{\circ}, 65^{\circ}$ and $31^{\circ}$ were carried out. The results were seen to be consistent up to $2.5 \mathrm{GHz}$, with slight deviations above that, which are mainly as a result of multiple reflections along the line resulting in loss ripples.

The DC resistance of the stitched transmission line with three different stitch angles $85^{\circ}, 65^{\circ}$ and $31^{\circ}$ corresponding to the number of stitches 60,90 and 162 were computed and a mathematical relationship was derived for computing the DC resistance of the stitch transmission line for any given number of stitches. The DC resistance computed results of $25.6 \Omega, 17.3 \Omega$ and $13.1 \Omega$, for $31^{\circ}, 65^{\circ}$ and $85^{\circ}$ stitch angles, indicated an increase in DC resistance of the stitch with a decrease in stitch angle which gives rise to an increase in a number of stitches. The transfer impedance of the stitched transmission line was also computed at low frequency $(<1 G H z)$ to be $Z_{T}=(0.24+j 1.09) \Omega$, with the result showing the effectiveness of the shield of the stitched transmission line at low frequency $(<1 \mathrm{GHz})$.

Keywords - Scattering Parameters; Stitched Transmission lines; Wearable Devices; Conductive Threads; DC resistance; Stitch angle; Multiple Reflections; Loss Ripples 


\section{Dedication}

To Stella for her enduring support, understanding, and love; and to my lovely kids Kerenhappuch and Malachi for their sweet love and being the source of my joy, forever grateful to JEHOVAH for you all 


\section{Acknowledgements}

First, I would like to give my utmost gratitude and sincere praise to JEHOVAH for HIS limitless blessings and grace throughout my program.

I gratefully acknowledge my supervisors: Dr. James Flint and Mr. Robert Seager, for their irreplaceable advice, guidance and shared knowledge throughout my Ph.D. I would also like to thank Dr. Chinthana Panagamuwa and Dr. Akram Alomainy for their shared experience and knowledge towards completing my thesis.

I acknowledge use of the facilities and the assistance of Keith Yendall in the Loughborough Materials Characterisation Centre.

I would also like to express my appreciation to Dr. Aris Tsolis, Duarte Fonseca and Dr. Shiyu Zhang for the invaluable assistance with the measurements.

I am also indebted to Tertiary Education Trust Fund, Nigeria (TETFUND) and Kaduna State University Kaduna, Nigeria (KASU) for their support during the years of my Ph.D.

Last but not least, I would like to thank my wife and two lovely kids, Stella, Keren-happuch and Malachi, my parents Hon. Daniel Hyuk and Grace Daniel, my family members, my inlaws, friends, and colleagues from Loughborough University and Kaduna State University for their encouragements, understanding and patience during the years of my $\mathrm{Ph} . \mathrm{D}$. 


\section{List of Publications}

1. E. W. Hurd, J. A. Flint and I. H. Daniel, "Shape-memory alloys in reconfigurable antennas", Antennas and Propagation Conference (LAPC), 2014 Loughborough, vol., no., pp.363,367, 10-11 Nov. 2014, doi: 10.1109/LAPC.2014.6996398

2. T. R. Lovett, J. A. Flint, D. S. Fonseca and I. H. Daniel, "Novel broadband antenna for wearables", Antennas and Propagation Conference (LAPC), 2014 Loughborough, vol., no., pp.178,181, 10-11 Nov. 2014, doi: 10.1109/LAPC.2014.6996350

3. I. H. Daniel, J. A. Flint and R. Seager, "Stitched Transmission Lines for Wearable RF Devices", Microwave and Optical Technology Letters, Vol. 59, No. 5, 2017, DOI:10.1002/mop.30461 


\section{Acronyms}

CST

Computer Simulation Technology

HIS

Information Handling Services

CPW

Coplanar Waveguide

EM

Electromagnetic

VNA

Vector Network Analyser

RG

Radio Grade

RF

Radio Frequency

DC

Direct Current

$\mathrm{AC}$

Alternating Current

EMI

Electromagnetic Interference

SEM

Scanning Electron Microscope

EMSE

Electromagnetic Shielding Efficiency

PTFE

Polytetrafluoroethylene

PE

Polyethylene

PTF

Polymer Thin Film

EM

Electromagnetic

EMI

Electromagnetic Interference

EMC

Electromagnetic Compatibility

EMS

Electromagnetic Susceptibility

PIFA

Planar Inverted Antenna

NFCA

Near-Field Communications Antenna 
$d B$

$T_{X_{2}}$

$C W+T_{2}$

$C W+T_{3}$

$C W+T_{4}$
Decibels

Stitched transmission line with stitch length at $2 \mathrm{~mm}$

Stitched transmission line with copper Wire + Conductive Thread with Stitch Length at $2 \mathrm{~mm}$

Stitched transmission line with copper Wire + Conductive Thread with Stitch Length at $3 \mathrm{~mm}$

Stitched transmission line with copper Wire + Conductive Thread with Stitch Length at $4 \mathrm{~mm}$ 


\section{List of Symbols}

Symbol Meaning

Unit

$\boldsymbol{R}$

Resistance per meter

$\Omega / \mathrm{m}$

$\boldsymbol{L}$

Inductance per meter

$n H / m$

$\boldsymbol{G}$

Conductance per meter

$S / m$

C

Capacitance per meter

$p F / m$

$D$

Separation between two cylindrical conductors

$m m$

$J$

Current density

$A m^{-2}$

$d$

Depth from surface

$m m$

$f$

Frequency

$G H z$

c

Free space velocity of light

$\mathrm{m} / \mathrm{s}$

$a$

Inner cylindrical conductor radius

$m m$

$b$

Outer conductor radius

$m m$

c

Dielectric radius

$m m$

w

Plate width

$m m$

$n$

Node (iteration) number

$g$

Hole width

$m m$

$h$

Height of cable

$m m$

$J_{S}$

Surface current density

$A m^{-2}$

$P_{x}$

Pitch

$m m$

$E_{i}$

Incident electric field

$N C^{-1}$

$E_{t}$

Transmitted electric field

$N C^{-1}$ 


\begin{tabular}{|c|c|c|}
\hline$H_{i}$ & Incident magnetic field & $T$ \\
\hline$H_{t}$ & Transmitted magnetic field & $T$ \\
\hline$R_{S}$ & Resistance of shield & $\Omega$ \\
\hline$P_{t}$ & Total power & $W$ \\
\hline$P_{i}$ & Incident power & $W m^{-2}$ \\
\hline$P_{r}$ & Reflected power & $W m^{-2}$ \\
\hline$S_{e f f}$ & Shielding effectiveness & $d B$ \\
\hline$T_{S}$ & Stitch tension & $N$ \\
\hline$T_{X}$ & Transmission line & - \\
\hline$W_{S}$ & Stitch width & $m m$ \\
\hline$L_{S}$ & Stitch length & $m m$ \\
\hline$N_{S}$ & Number of stitches & - \\
\hline$N_{w}$ & Number of wires & - \\
\hline$C_{c}$ & Number of carriers crossing per unit length & - \\
\hline$D_{O}$ & Outer diameter of dielectric & $m m$ \\
\hline$D_{S}$ & Diameter of stitch transmission line before stitching & $m m$ \\
\hline$D_{b}$ & Diameter of the metal braid shield & $m m$ \\
\hline$D_{m}$ & Mean braid diameter & $m m$ \\
\hline$Z_{0}$ & Characteristic impedance & $\Omega$ \\
\hline$R_{0}$ & Real part of Characteristic impedance & $\Omega$ \\
\hline$X_{0}$ & Imaginary part of Characteristic impedance & $\Omega$ \\
\hline$d_{s}$ & Plate separation & $m m$ \\
\hline
\end{tabular}




\begin{tabular}{|c|c|c|}
\hline$d_{p}$ & Plate thickness & $\mathrm{mm}$ \\
\hline$d_{w}$ & Diameter of a single wire & $\mathrm{mm}$ \\
\hline$l_{c t}$ & Conductive Thread length & $\mathrm{mm}$ \\
\hline$h_{S D}$ & Thickness of dielectric substrate (Denim) & $\mathrm{mm}$ \\
\hline$h_{S F}$ & Thickness of dielectric substrate (Felt) & $\mathrm{mm}$ \\
\hline$h_{r}$ & Radial spindle separation & $\mathrm{mm}$ \\
\hline$\Delta z$ & Length & $\mathrm{mm}$ \\
\hline$\rho$ & Resistivity & S. $m$ \\
\hline$\sigma$ & Conductivity & $S / m$ \\
\hline$\delta$ & Skin depth & $\mu m$ \\
\hline$x$ & Coverage (optical) & - \\
\hline$\omega$ & Radian frequency & $\operatorname{rads}^{-1}$ \\
\hline$\alpha_{w}$ & Weave angle & $\circ$ \\
\hline$\lambda_{C}$ & Cut-off wavelength & $\mathrm{mm}$ \\
\hline$f_{c}$ & Cut-off frequency & $H z$ \\
\hline$\mu_{0}$ & Permeability of free space & $H / m$ \\
\hline$\mu_{r}$ & Relative permeability & - \\
\hline$\varepsilon_{0}$ & Permittivity of free space & $F / m$ \\
\hline$\varepsilon_{r}$ & Relative permittivity & - \\
\hline$\varepsilon_{r}^{\|}$ & Complex part of relative permittivity & - \\
\hline$\varepsilon_{r}^{\prime}$ & Real part of relative permittivity & - \\
\hline $\tan \delta$ & Loss tangent & - \\
\hline
\end{tabular}




\begin{tabular}{|c|c|c|}
\hline$D_{k}$ & Dielectric constant & - \\
\hline$\lambda$ & Wavelength & $\mathrm{mm}$ \\
\hline$\alpha_{x}$ & Stitch angle & $\circ$ \\
\hline$\beta_{X}$ & Weave/ braid angle & $\circ$ \\
\hline$\gamma$ & Propagation constant & $m^{-1}$ \\
\hline$\alpha$ & Attenuation constant & $N m^{-1}$ or $d B^{2} m^{-1}$ \\
\hline$\beta$ & Phase constant & - \\
\hline$k$ & Wave number & - \\
\hline$\Gamma$ & Reflection Coefficient & - \\
\hline$s$ & Standing wave ratio & - \\
\hline$u$ & Wave velocity & $m s^{-1}$ \\
\hline
\end{tabular}




\section{List of Figures}

Figure 1.1 Wearable Technology: Major segments and players

Figure 1.2 Single Layer P-FCB system manufacturing process

Figure 2.1 Transmission line as a two-port network

Figure 2.2 Twin-wire parallel conductor transmission line

Figure 2.3 Coaxial cable transmission line

Figure 2.4 Parallel plate transmission line; plate width $\mathrm{w}$ is large compared with the separation $d_{s}$

Figure 2.5 Equivalent circuit model of a lossy transmission line

Figure 2.6 Equivalent circuit model of a lossless transmission line

Figure 2.7(a) Input impedance due to a line terminated by a load; (b) equivalent circuit for finding $V_{0}$ and $I_{0}$ in terms of $Z_{\text {in }}$ at the input

Figure 2.8 Conductive paths made of copper wires in a fabric

Figure 2.9 Screen Printed Sample of CPWs on Evolon

Figure 2.10 Textile transmission lines in which the conductive paths are made from a different electro conductive flat textile

Figure 2.11 Conductive thread transmission line created through digital embroidery in jig above FR4 substrate

Figure 2.12 (a) Formed by twisting many thin elastic silver plated nylon fibres together (b) contains 60 copper fibres, each of which has a diameter of $40 \mu \mathrm{m}$, (c) and (d) are composite threads of insulating and metallic fibres where both composite threads are created by spinning $40 \mu \mathrm{m}$ silver plated copper fibres around a nonconductive core which is composed of multiple nonconductive fibres and (e) is made up of single silver plated copper fibre with a diameter of $40 \mu \mathrm{m}$ 
Figure 2.13 (a) Conductive thread from Light Stiches® (b) Colour image with Highdynamic-range imaging (HDR) of the Conductive thread from Light Stiches ${ }^{\circledR}$ using the Olympus LEXT Laser Confocal Microscope at $100 \mu \mathrm{m}$

Figure 4.1 (a) Geometry of the stitched transmission line (b) Zoomed in view of fabricated stitched transmission line

Figure 4.2 (a) Singer All-Purpose Snap-On Presser Foot (b) Top, (c) bottom, (d) rear and (e) side view of the novel presser foot

Figure 4.5 Schematic illustration of the stitch angle (a) Stitch angle and (b) Three different stitch angles

Figure 4.6 Perspective view of counterwound helices and planar development of the cylindrical surface

Figure 4.7 3-D view of stitched transmission line design with CST studio suite ${ }^{\circledR}$

Figure 4.8 Cross section of the stitched transmission line

Figure 4.9 Figure 4.9 S-Parameters of stitched transmission line with stitch angles $85^{\circ}, 65^{\circ}$ and $31^{\circ}$ corresponding to stitch lengths $1.7 \mathrm{~mm}, 1.5 \mathrm{~mm}$ and $1.2 \mathrm{~mm}$ respectively, using CST Microwave Studio Suite®

Figure 4.10 Plot of $\boldsymbol{S}_{\mathbf{1 1}}^{\mathbf{2}}+\boldsymbol{S}_{\mathbf{2 1}}^{\mathbf{2}}$ against frequency with stitch angles $85^{\circ}, 65^{\circ}$ and $31^{\circ}$ 4.17

Figure 4.11 Cross section of (a) Square coaxial line (b) General rectangular coaxial line (c) Coaxial stripline (d) Elliptical coaxial line whose conductors are confocal ellipses (e) Coaxial line with outer conductor of elliptical cross section (f) Polygonal coaxial line with cylindrical centre conductor

Figure 4.12 Circuit representation of the lossy stitched transmission line 
Figure 4.13 Top view of cross section of 3D fields on 2D plane of the stitched transmission line with stitch angle at $85^{\circ}$ used to compute its electrical length

Figure 4.14 Top view of cross section of 3D fields on 2D plane of the stitched transmission line with stitch angle at $65^{\circ}$ used to compute its electrical length

Figure 4.15 Top view of cross section of 3D fields on 2D plane of the stitched transmission line with stitch angle at $31^{\circ}$ used to compute its electrical length

Figure 5.1 Measurement set up with Anritsu MS46524A 7GHz Vector Network Analyser

Figure 5.2 Measured S-Parameters of stitched transmission line with $C W_{-} C T_{-} L 2, C T_{-} L 2$ and $S I M \_L 2$

Figure 5.3 Plot of $\boldsymbol{S}_{\mathbf{1 1}}^{\mathbf{2}}+\boldsymbol{S}_{\mathbf{2 1}}^{\mathbf{2}}$ against frequency of stitched transmission line with CW_CT_L2, CT_L2 and SIM_L2

Figure 5.4 Measured S-Parameters of stitched transmission line with $C W_{-} C T_{-} L 3, C T_{-} L 3$ and $S I M \_L 3$

Figure 5.5 Plot of $S_{11}^{2}+S_{21}^{2}$ against frequency of stitched transmission line with CW_CT_L3, CT_L3 and SIM_L3

Figure 5.6 Measured S-Parameters of stitched transmission line with CW_CT_L4, CT_L4 and SIM_L4

Figure 5.7 Plot of $S_{11}^{2}+S_{21}^{2}$ against frequency of stitched transmission line with CW_CT_L4, CT_L4 and SIM_L4

Figure 5.8 Fabricated stitched transmission line with stitch angles of (a) $85^{\circ}$ (b) $65^{\circ}$ and (c) $31^{\circ}$

Figure 5.9 Measured S-Parameters of stitched transmission line with stitch angle and length at $31^{\circ}$ and $1.2 \mathrm{~mm}$ respectively

Figure 5.10 Measured S-Parameters of stitched transmission line with stitch angle and length at $65^{\circ}$ and $1.5 \mathrm{~mm}$ respectively

Figure 5.11 Measured S-Parameters of stitched transmission line with stitch angle and length at $85^{\circ}$ and $1.7 \mathrm{~mm}$ respectively 
Figure 5.12 Plot of $\boldsymbol{S}_{\mathbf{1 1}}^{\mathbf{2}}+\boldsymbol{S}_{\mathbf{2 1}}^{\mathbf{2}}$ against frequency with stitch angles $85^{\circ}, 65^{\circ}$ and $31^{\circ}$

Figure 5.13 Three different stitch types used (a) Double Overlock stitch (b) Flatlock stitch and (c) Ric-Rac stitch

Figure 5.14 Zoomed in view of constructed stitched transmission line with three different stitch types

Figure 5.15 Measured S-Parameters of stitched transmission line with three different stitch types

Figure 5.16 Plot of $\boldsymbol{S}_{\mathbf{1 1}}^{\mathbf{2}}+\boldsymbol{S}_{\mathbf{2 1}}^{2}$ against frequency with three different stitch types

Figure 5.17 Stitched transmission line with different curved bending angles at (a) $90^{\circ}$ (b) $180^{\circ}$

Figure 5.18 Measured S-Parameters of stitched transmission line with two different bending conditions and a straight stitched line compared with a straight simulated line

Figure 5.19 Plot of $\boldsymbol{S}_{\mathbf{1 1}}^{\mathbf{2}}+\boldsymbol{S}_{\mathbf{2 1}}^{\mathbf{2}}$ against frequency with two different bending conditions and a straight stitched line compared with a straight simulated line

Figure 5.20 Measured S-Parameters of Washed and Unwashed stitched transmission line 5.25

Figure 5.21 Plot of $\boldsymbol{S}_{\mathbf{1 1}}^{\mathbf{2}}+\boldsymbol{S}_{\mathbf{2 1}}^{\mathbf{2}}$ against frequency with two different bending conditions

Figure 5.22 Cutoff section of the washed thread from the stitched transmission line

Figure 5.23 Scanned image of unwashed stitched transmission line

Figure 5.24 Scanned image of unwashed stitched transmission line

Figure 5.25 Schematic diagram of On-body measurement on the stitched transmission line

Figure 5.26 On-body measurements on the stitched transmission line

Figure 5.27 Off and On body measurements on the stitched transmission line

Figure 5.28 Plot of $\boldsymbol{S}_{\mathbf{1 1}}^{\mathbf{2}}+\boldsymbol{S}_{\mathbf{2 1}}^{\mathbf{2}}$ against frequency for off and On body measurements on the stitched transmission line

Figure 5.29 Stitched transmission line with Denim and Felt used as substrates

Figure 5.30 Comparison of S-Parameters between Denim and Felt used as substrates

Figure 5.31 Plot of $\boldsymbol{S}_{\mathbf{1 1}}^{2}+\boldsymbol{S}_{\mathbf{2 1}}^{2}$ against frequency with Denim and Felt as substrates 
Figure 5.32 Measured S-Parameters of stitched transmission line with stitch angle $85^{\circ}$ (stitch length, $L=1.7 \mathrm{~mm}$ ) 5.41

Figure 5.33 Measured S-Parameters of stitched transmission line with stitch angle $65^{\circ}$ (stitch length, $\left.L=1.5 \mathrm{~mm}\right)$

Figure 5.34 Measured S-Parameters of stitched transmission line with stitch angle $31^{\circ}$ (stitch length, $L=1.2 \mathrm{~mm}$ ) 5.43

Figure 5.35 Equivalent electrical model of the stitched transmission line $(N=1) \quad 5.46$

Figure 5.36 Equivalent electrical model of the stitched transmission line $(N=2) \quad 5.46$

Figure 5.37 Equivalent electrical model of the stitched transmission line $(N=3) \quad 5.47$

Figure 5.38 Equivalent electrical model of the stitched transmission line $(N=M) \quad 5.47$

Figure 5.39 Cross-sectional view of a conductor with (a) non-energized, (b) low frequency, (c) middle frequency and (d) high frequency 


\section{List of Tables}

Table 2.1 Modes in Transmission line

Table 4.1 CST Studio Suite design dimensions for stitch angle of $85^{\circ}$

Table 4.2 Analytical curve of the helices

Table 4.3 Termination of the helices

Table 5.1 Measured DC Resistance for Double Overlock stitch, Flatlock stitch and Ric-Rac stitch

Table 5.2 DC resistance of the shield of stitched transmission line before and after washing

Table 5.3 Properties of the human tissues

Table 5.4 Summary of dielectric properties of nonconductive fabrics

Table 5.5 Simulated Characteristic Impedance of the Stitched Transmission Line with Different Cross Section Dimensions

Table 5.6 DC resistance of the shield of stitched transmission line

Table 5.7 Skin depth $(\delta)$ for some common materials 


\section{Contents}

Abstract i-ii

$\begin{array}{ll}\text { Dedication } & \text { iii }\end{array}$

Acknowledgements iv

List of Publications $\quad$ v

Acronyms vi-vii

List of Symbols $\quad$ viii-xi

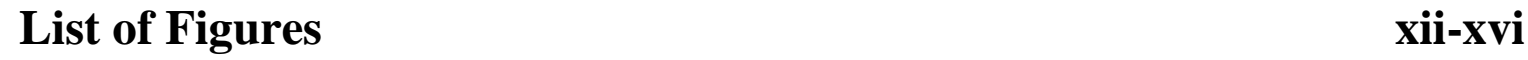

$\begin{array}{ll}\text { List of Tables } & \text { xvii }\end{array}$

\section{CHAPTER 1: Introduction}

1.1. Background 1.1

1.2. Wearable Technology 1.2-1.3

1.2.1. Wearability in Wearable Technology $1.3-1.4$

1.2.1.1. Flexibility 1.4

1.2.1.2. Mobility and comfortability $\quad 1.5$

1.2.1.3. Durability 1.5

1.2.1.4. Thermal Management 1.5

1.2.1.5. Moisture and Dust Management 1.6

1.2.1.6. Garment care 1.6

1.2.1.7. Sizing and Fit $1.6-1.7$

1.3. Wearable Communication Systems 1.7

1.4. Research Motivation $\quad 1.7-1.8$

1.5. Research Objectives 1.8

1.6. Novel Contribution of the Thesis $1.8-1.9$

1.7. Thesis Outline $1.9-1.10$

Reference $1.11-1.12$ 


\section{CHAPTER 2: Transmission Line and Antennas}

$\begin{array}{ll}\text { 2.1. Introduction } & 2.1-2.3\end{array}$

2.2. Transmission Line Parameters 2.3-2.5

2.3. Transmission Line Equations $2.6-2.10$

2.4. Input Impedance, Voltage Standing Wave Ratio, and Power 2.10-2.14

2.5. Transmission Line Loss and Attenuation 2.15-2.16

2.6. Wearable Transmission Lines 2.16

2.6.1. Methods of Implementation of Wearable Transmission Lines 2.17-2.21

$\begin{array}{ll}\text { 2.6.2. Conductive Threads for Signal Transmission } & 2.21\end{array}$

2.7. Fabrication of Conductive Threads $\quad 2.22$

$\begin{array}{lr}\text { 2.8. Conductive Threads from Light Stitches }{ }^{\circledR} & 2.23-2.24\end{array}$

2.9. Conclusion 2.26

$\begin{array}{lr}\text { References } & 2.27-2.29\end{array}$

\section{CHAPTER 3: Analysis on the Shield of a Coaxial Cable}

3.1. Coaxial Cable

3.1.1. Role of the shield

3.2. Theoretical Evaluation of Cable Shielding

3.2.1. Shielding Effectiveness of a Braided Cable Shield

3.2.2. Transfer Impedance of a Braided Cable Shield

3.2.2.1. Vance Model

3.2.2.2. Tyni Model

3.2.2.3. Kley Model

3.3. Application of the Kley model to the stitched Transmission Line

3.4. Conclusion

Reference 


\section{CHAPTER 4: Numerical Modelling and Fabrication of the Stitched Transmission Line for Broadband Operations}

4.1. Stitched Transmission Line for Wearable RF Devices

4.1.1. Novel Presser Foot

4.1.2. Stitch Angle

4.2. Full Field Model with CST Microwave Studio Suite ${ }^{\circledR}$

4.2.1. Characteristic Impedance of the Stitched Transmission Line

4.3. Conclusion

Reference

$4.32-4.34$

\section{CHAPTER 5: Experimental Measurement on the Stitched Transmission} Line

5.1. Introduction to RF Measurement on the Stitched Transmission Line

$5.1-5.2$

5.2. Stitched Transmission Line with Conductive Threads and Copper Wire

5.3. Stitched Transmission Line with Conductive Threads

5.3.1. Stitched Transmission Line with Three Different Stitch Angles

$5.10-5.16$

5.3.2. Stitched Transmission Line with Different Stitch Types

5.3.3. Stitched Transmission Line with Different Curved Bending Angles

5.3.4. Stitched Transmission Line Subjected to Washing Cycles

5.3.5. Stitched Transmission Line On-body Measurements

5.4. Design Sensitivity to Manufacturing Tolerance

5.4.1. Dielectric properties of fabrics

$5.34-5.36$

5.4.2. Stitched Transmission Line with Different Substrates

$5.36-5.39$

5.4.3. Sensitivity of the Characteristic Impedance with Changes in Cross Sectional Dimensions of the Stitched Transmission Line

$5.39-5.40$

5.4.4. Repeatability of the Stitched Transmission Line $5.40-5.43$

5.5. Shielding Effectiveness of the Stitched Transmission Line 
5.5.3. Transfer Impedance of the Stitched Transmission Line

5.6. Conclusion

Reference

\section{CHAPTER 6: Conclusions and Future Work}

6.1. Summary

$6.1-6.2$

6.2. Main Contributions

$6.2-6.3$

6.3. Industrial Applications

6.3

6.4. Future Research

$6.3-6.4$

Reference

6.5

\section{Appendix}
A. 8216 Coax - RG-174/U Type
A.1-A.3
B. Plug BNC
B. 1
C. Newton-Raphson Method
C. $1-$ C. 2
D. Taylor Series Expansion - Function for one variable
D. 1
E. Quadratic Equation
E. 1 


\section{Chapter 1}

\section{Introduction}

\subsection{Background}

Today's textiles materials have come to find lots of applications in virtually all our daily events. Besides wearing apparels all the time and being constantly surrounded with textiles in virtually all of our environments, the integration of multifunctional values in such a common material has become a special area of interest in recent years with the use of electronic components on textile materials becoming a major growth area. Textile transmission lines have been developed and used as means for transmitting signals to and from wearable devices over the last decade, with extensive characterization for use in wearable computing applications. This thesis focuses on the integration of transmission lines into apparels, specifically on using the idea of a braided coaxial cable to develop a stitched transmission line that is capable of transmitting signals within (but not limited to) the frequency range of $0.04-4 \mathrm{GHz}$. The frequency range of $0.04-4 \mathrm{GHz}$ was chosen because of its vast applications in GSM, mobile communications, Microwave devices and communications, mobile phones broadband etc. The stitched transmission line functions like the conventional transmission lines, but differs from it as it is wearable, lightweight, flexible, washable, robust and comfortable. Generally, in designing smart apparels, it is vital that design requirements such as the operational reliability, controllability, convenience, ergonomics and robustness be met in order to meet the desires of the target consumers [1.1]-[1.2].

The increase in demand and rapid growth of miniaturization of electronic devices over the last decade had also led to the development of devices that are mobile, convenient and assessable both in their physical form and applications. The trend of electronics

miniaturization, both in military and consumer applications challenges board assembly materials, processes and reliability. This growth along with advances in wearable devices has led to the development of devices which can either be carried by the user or directly integrated into apparels known as smart textiles envisioned for continuous or situation appropriate accessibility and use [1.3]-[1.4]. 


\subsection{Wearable Technology}

Wearable technology can be viewed as the combination of computing and wireless technologies with apparels, jewelry and other accessories. It is also a term used to describe many different forms of body mounted technology; they can be anything from a headband to a smart watch (Apple Watch®, Samsung Galaxy Gear®, Fitbit ${ }^{\circledR}$ and Pebble smartwatch®), from smart apparels to smart shoes (Apple Smart Shoes ${ }^{\circledR}$ ) and technology implantable or embedded skin (devices that can be directly introduced into the human body to modify, enhance or heal in ways that non-embedded devices cannot) [1.5]. Usually, there are three categories of wearable technology electronics; these include electronics that are worn directly, those integrated into apparels (Smart Textiles) and lastly those that are used as accessories on apparels. These routinely worn devices are enabled with portable computing and wireless networking, transforming them into linked devices that can provide features such as monitoring, computing etc. A pictorial representation of this is given in Fig.1.1.

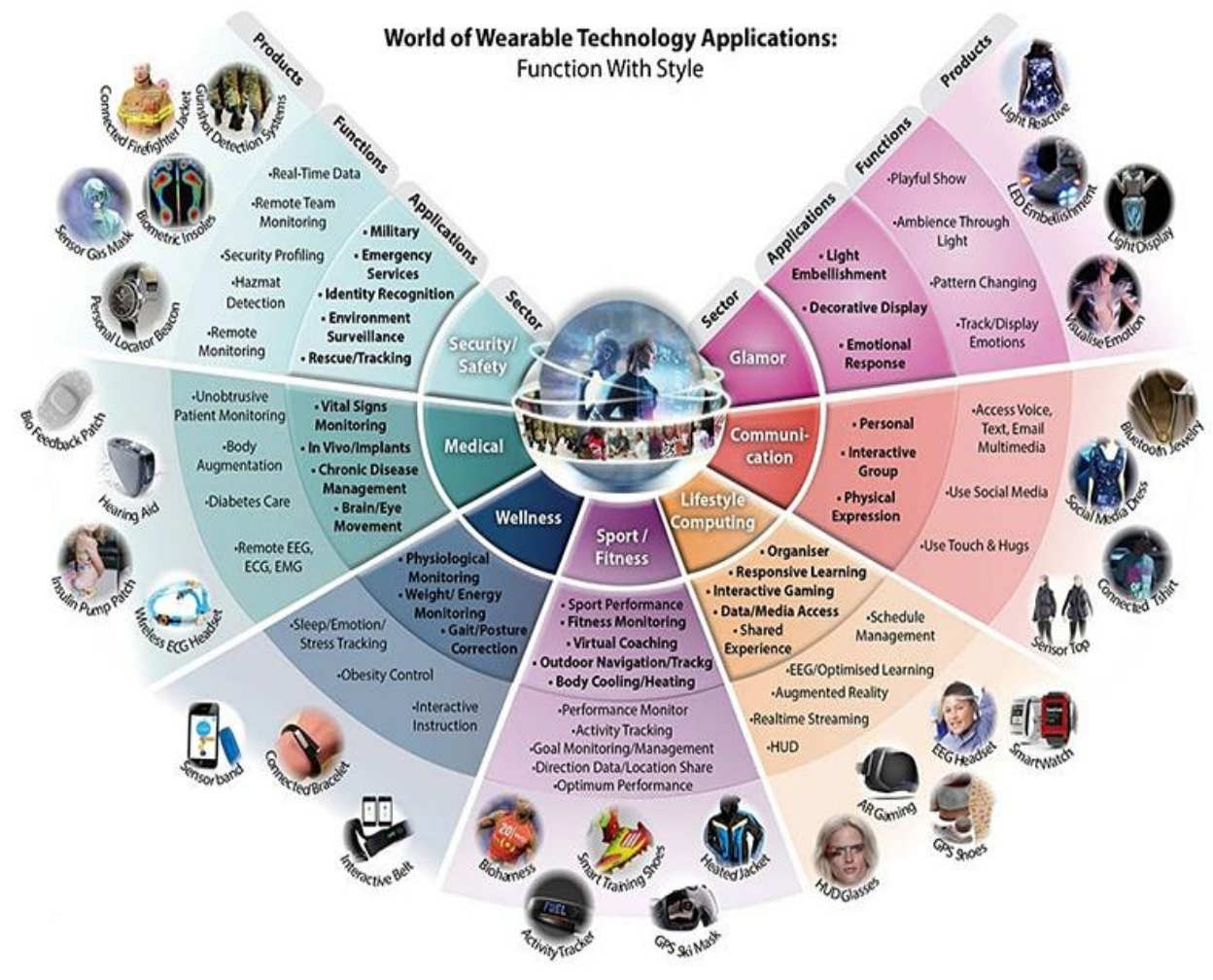

Figure 1.1 Wearable Technology: Major segments and players after Beecham Research [1.6] 
The HIS (Information Handling Services) Electronic and Media estimated that the world market for wearable technology reached a total of $\$ 8.5$ billion in revenues in 2012; shipping 96 million devices with a forecast for 210 million shipments projected for 2018, driving \$30 billion in revenue [1.7]. The fitness market dominated by players like Adidas, Nike, FitBit, Jawbone etc. is estimated to be about $\$ 2-\$ 3$ billion [1.8]. Equally, according to a new market research report, "Wearable Technology Market by Product (i.e. Wristwear, Eyewear, Footwear, Neckwear, Bodywear, and Others), Application (Consumer Electronics, Healthcare, Enterprise \& Industrial, and Others), Type (Smart Textile, Non-Textile), and Geography - Global Forecast to 2020", the overall market for wearables is forecast to reach $\$ 31.27$ billion at a compound annual interest rate of $17.8 \%$ between 2015 and 2020 [1.9]. Equally, with about $41 \%$ compound annual growth rate, ABI Research forecasts a growth in wearable devices in sports from 20.77 million devices in 2011 to 169.5 million devices by 2017 [1.10]. All these clearly show a clear demand for wearable technologies and a need for continued research into the methods of constructing and maintaining such devices.

\subsubsection{Wearability in Wearable Technology}

Wearability is the durability of clothing under normal wear or the ability of apparel to withstand prolonged wear, while wearable refers to the use of the human body as a support environment for the product.

A product is said to be wearable if it is suitable for wear or can be worn on the body comfortably and does not restrict the user [1.11]. A wearable device has to be robust to the environmental pressures existing on the human body. The human body is dynamic and its form is diverse and changing. Hence wearable designs that appreciate these dynamics result in product wearability. With today's wearable devices access to information is no longer restricted to static environment of the office desktop and personal computer as well designed mobile and wearable devices offer more effective and convenient ways for people to relate to this information [1.12].

In designing wearable devices consideration has to be given to the cognitive interaction between the user and the apparel. Apart from cognitive considerations, wearable technology also introduces new physical and psychological variables. In subsequent sections, several functional clothing design areas of concern will be examined with respect to the additional 
variables and requirements of wearable technology. These include flexibility, mobility, durability, thermal and moisture management, garment care and sizing and fit.

\subsubsection{Flexibility}

To make apparels wearable, there's a need to make electronic components integrated or mounted on them to be as flexible as possible. Flexible electronics also known as flex circuits' technology where electronic devices are mounted on flexible PCBs are used. This allows the PCBs to adapt to the desired shape or to flex during use. Here the design is such that the components are assembled in bend and twist stabilized regions. The planar fashionable circuit board (P-FCB) is a good example of this, which features a soft and flexible impression just as regular apparels [1.13].
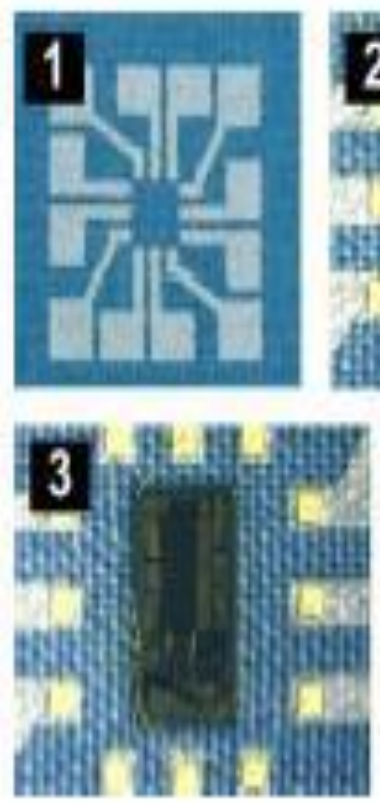
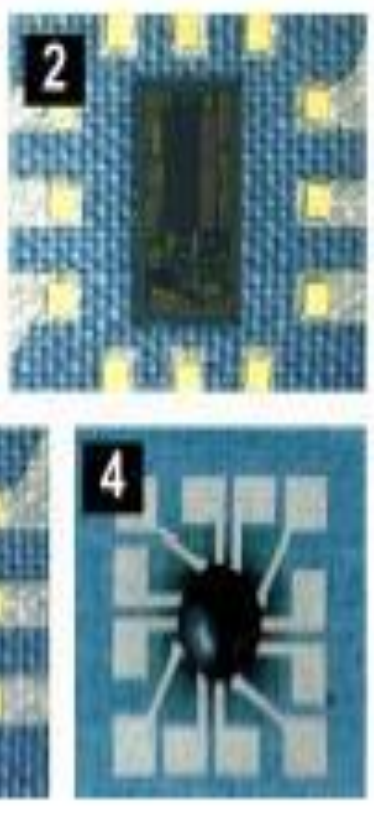

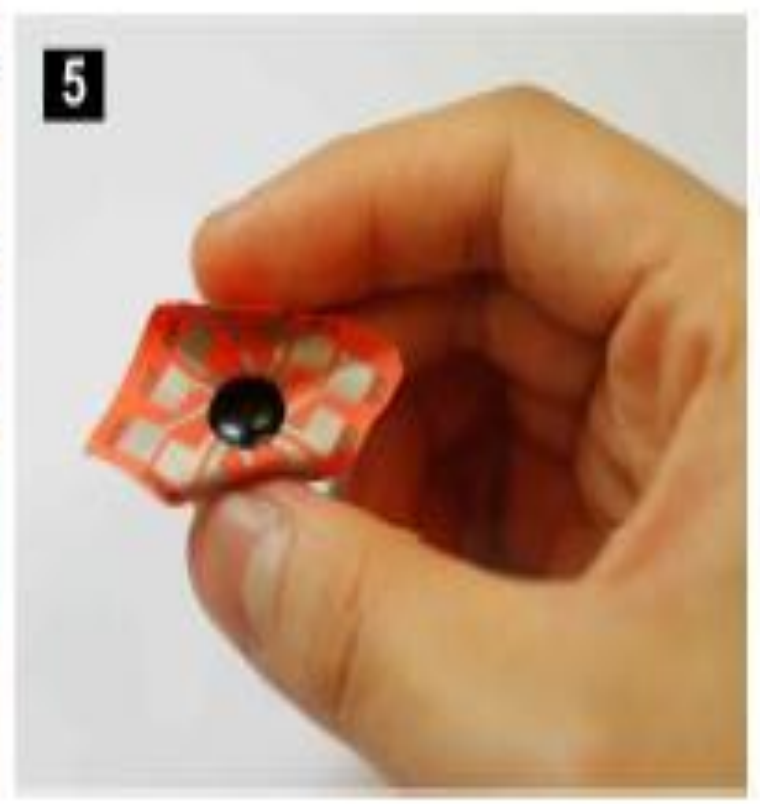

Figure 1.2 Single-layered P-FCB system manufacturing process [1.13] 


\subsubsection{Mobility and Comfortability}

It is important that wearable devices are designed such that they can produce physical comfort, support or ease as users feeling enough comfort while using the devices no longer sense the presence of the device after wearing it for a while [1.14].

The biggest and heaviest part of the wearable device is the energy supply [1.15]. Also in wearable technology designs where resources do not permit the custom design of computing units, bulky devices mounted on the body are quite often used. Having these devices integrated into apparels or mounted on the body ought to be done in such a manner that it does not totally interfere with natural movements thus becoming a burden to the wearer. As such, designs for such wearable devices ought to take into cognisance situation-specific movements desired for achieving specific tasks.

\subsubsection{Durability}

The ability to withstand wear, pressure or damage is also a challenge to the development of wearable electronic components. They must also be able to withstand the human environment they find themselves in, as it is not an ideal environment for them. Proper positioning of these devices and stabilizers are possible solutions to this problem.

\subsubsection{Thermal Management}

Wearable electronics present some unique thermal design challenges for both the package and the whole system by adding the heat produced by certain electronic components to that produced by the body. Operating temperature is not set by reliability requirements alone. Wearable electronics in contact with the skin should have a comfortable thermal operating range as the device also has to maintain a certain comfort level. An ideal operating temperature of $37^{\circ} \mathrm{C}\left(98.6^{\circ} \mathrm{F}\right)$ needs to be maintained for electronics placed in direct contact with the skin as any figure beyond this could cause a lot of discomfort to the wearer. Without thermal management, the human skin could act as a heat sink to dissipate this thermal energy. Also, conductive transfer from components can be facilitated through heat sinks attached to them [1.16]. 


\subsubsection{Moisture and Dust Management}

Most PCBs made up of water-resistant plastics and resins can hold heat and moisture and when placed close to the body could cause a lot of discomforts. However, this can be managed through the use of breathable or wicking fabrics, which are fabrics that draw moisture away from the body by letting in air and sweat out [1.17]. There's also the need to protect wearable devices through partial or full encapsulation from dust, perspiration, precipitation, spilled liquids and extreme moisture during laundering to preserve functionality.

\subsubsection{Garment care}

Washing, drying and smoothing clothes are means of taking care of apparel that we wear. Washing these apparels could either be through machine wash or hand wash. With the machine wash, detergents or liquid soaps alongside fabric softeners are used while soap bars and detergents are sometimes used for the hand wash. Washing and drying these apparels could cause a lot of damage to the electronic technology. However, it is also worth mentioning here that some electronics components can withstand washing and drying provided the power source is detached or they are not been powered by some power source. For some devices, however, the temperature at which the washing is done is more of an issue, as the temperatures these devices can withstand vary from one device to the other.

Furthermore, care has to be taken when smoothing these apparels using either pressing irons or steamers to avoid contact with some of the electronic components as these could also cause some impairment to the electronic components integrated or mounted on the apparels. Possible solutions to these problems include the use of wearable devices that can be enclosed or detached from the apparel.

\subsubsection{Sizing and Fit}

This is a crucial area if electrical and electronic devices are to be integrated into apparels or mounted on the body. Usually, the sizing of the body which involves taking measurements from areas like the neck, sleeves, chest, waist, hips, and inseam is done for proper fit. Depending on the type of apparel in use, one can have next to skin fit (which conforms to the body's contours and are typically base layers), active fit (tailored to fit closer to the body and may be worn over baselayers, under outer layers, or as stand-alone pieces), standard fit 
(which is neither formfitting nor loose and may be worn over base and mid layers) and relaxed fit (drapes loosely on the body; relaxed fit items are typically outer layers) [1.18]. The wearable device to be integrated or mounted on apparel will determine the type of fit required.

\subsection{Wearable Communication System}

On-body communication can be wired or wireless, which could include infrared, embedded wiring and Bluetooth technology. However, most wearable devices make use of Bluetooth to communicate, which can be inefficient because of how difficult it is for the signal to pass through a user's body and can cause an effect called "path loss" whereby a signal deteriorates as it travels between two wearable devices, as well as causing security concerns due to the distance the signal travels, with the user being susceptible to eavesdropping [1.19]. The use of textile transmission lines tends to moderate some of these deficiencies when transmitting RF signals between various parts of wearable communication systems.

\subsection{Research Motivation}

To connect wearable devices operating at radio frequencies, transmission lines which are efficient, light weight with low fabrication cost, flexible to withstand bending and stretching, and capable of providing shielding from the ingress and egress signals are vital components of such a system. Problems usually associated with wearable transmission lines include durability of the wearable transmission line and maintenance of the smart clothing which house all elements including the wearable transmission line. The need to develop a wearable transmission line to moderate some of these glitches requires a careful understanding of previous wearable transmission lines. However, with the scarce literature on wearable transmission lines, only a few authors [1.20]-[1.21] have investigated efficient low-profile structures which are achievable at low cost, with various variations investigated for application for frequencies up to a few GHz. A careful study of these problems necessitated the 
prerequisite to developing a stitched transmission line that will mitigate some of these glitches.

\subsection{Research Objectives}

The stitched transmission line should provide a transmission line that is wearable with good electrical characteristics such as low DC resistance, low reflection and resistive losses at radio frequencies.

The use of the textile transmission line comes with a couple of challenges which include sourcing of optimal materials to be used, the need for individual conductors to be insulated from each other, the effect of stitch types and densities, and the need to be able to withstand typical textile handling.

The main objectives of this research include:

- Model numerically a novel stitched transmission line within a frequency range of $0.04-4 G H z$.

- Develop a presser foot that will suite the requirement for the use of RG174 coaxial cable in the fabrication of the stitched transmission line

- Analyze numerically the DC resistance of three different stitch length (Stitch angles) of the stitched transmission line and computation of the transfer impedance of the stitched transmission line at low frequency $(<1 \mathrm{GHz})$.

- Characterize experimentally the stitched wearable transmission line

- Explore design features of the system and their impact on system and user performance

\subsection{Novel Contribution of the Thesis}

The novelties of this thesis are:

(1) The use of the idea of a braided coaxial cable to develop a stitched transmission line that is suitable for carrying RF signals to wearable devices within the frequency range of $0.04-4 G H z$

(2) Introduction of numerical modeling and simulation procedures for stitched wearable transmission lines using CST Microwave Studio Suite ${ }^{\circledR}$ 
(3) The design and fabrication of a novel presser foot to meet stitching requirements for the stitched transmission line

(4) Development of a mathematical relationship that can be used to compute the DC resistance of the shield for any given number of stitches

(5) Evaluation of the effect of the stitched parameters on the DC and RF performance of the stitched wearable transmission line

\subsection{Thesis Outline}

Chapter 1 provides a background introduction to the thesis. It also offers an overview of wearable technologies highlighting growths in wearable technology over the years alongside wearability as it applies to wearable devices. A brief summary of wearable communication systems, research objectives, motivation and the novelty of the thesis were also presented.

Chapter 2 will present the theoretical background of transmission lines with an overview of transmission line theory, review of some wearable transmission lines and the different methods of fabricating textile transmission lines. A brief introduction on conductive threads from Light Stitches ${ }^{\circledR}$ used in the construction of the stitched transmission line was also presented.

Chapter 3 will present an analysis of the braided coaxial cable with emphasis on the role of the shield, shielding effectiveness and its transfer impedance. These analyses are applicable to the stitched transmission line which can also be envisaged as a composite counter-wound helical structure [1.22]-[1.24] with a concentric conductor.

Chapter 4 offers the numerical modeling and construction of the stitched transmission line with the aid of a modified presser foot using three different stitch lengths corresponding to three stitch angles.

Chapter 5 presents experimental measurements and performance characteristics of the prototype model of the stitched wearable transmission line under different conditions such as stitch lengths (stitch angles), stitch types and curved bending angles. It also presents on-body measurements as well as its design sensitivity to manufacturing tolerance and its performance after subjecting it to washing cycles. Repeatability measurements were also carried out in this chapter. 
Finally, Chapter 6 highlights the main conclusions of this research, industrial applications and offer suggestions for further work. 


\section{REFERENCE}

1.1. M. Chedid, P. Leisner and I. Belov, "Experimental Analysis and Modelling of Textile Transmission Line for Wearable Applications," International Journal of Clothing Science and Technology, Vol. 19 N0. 1, pp. 59-71, 2007.

1.2. D. Cottet, J. Grzyb, T. Kirstein and G. Tröster, "Electrical Characterisation of Textile Transmission Lines," IEEE Transaction on Advance Packaging, Vol. 26, N0. 2, 182190, 2003

1.3. C. Baber, J. Knight, D. Haniff and L. Cooper, "Ergonomics of Wearable computers," Mobile Networks and Applications, vol.4, pp.15-21, 1991

1.4. P. S. Hall and Y. Hao, "Antennas and Propagation for Body-Centric Wireless Communications," Second Edition, pp.1, Artech House, 2012

1.5. Innovations The Rise of Wearables Future Gear [Online]. [Accessed: 12-February2017]. Available from: http://www.cleverism.com/rise-wearables-future-gear/

1.6. Wearable Technology Application Chart, [Online]. [Accessed 12-February-2017]. Available from: http://www.beechamresearch.com/download.aspx?id=36

1.7. S. Walker, "Wearable Technology - Market Assessment," IHS Electronics \& Media, 2013. [Online]. [Accessed: 12-February-2017]. Available from: http://cdn2.hubspot.net/hub/396065/file-2568104498-pdf/Blog_Resources/IHS-

Wearable-Technology.pdf?t=1427903372862

1.8. P. M. Vaikuntaraman, "Wearable Technology Market, Market Analysis - Trends \& Outlook," [Online] [Accessed 12-February-2017]. Available from: http://www.slideshare.net/PreethiMyla/wearable-technology-market

1.9. Wearable Technology Market worth 31.27 Billion USD by 2020, Markets and Markets [Online]. [Accessed: 12-February-2017]. Available from: http://www.marketsandmarkets.com/PressReleases/wearable-electronics.aspv

1.10. Wearable Sports and Fitness Devices Will Hit 90 Million Shipments in 2017, ABI Research, 2012. [Online]. [Accessed: 12-February-2017]. Available from: https://www.abiresearch.com/press/wearable-sports-and-fitness-devices-willhit-90-mi

1.11. Wearability, [Online]. [Accessed 12-February-2017]. Available from: http://dictionary.reverso.net/english-definition/wearability

1.12. F. Gemperle, C. Kasabach, J. Stivoric, M. Bauer, and R. Martin, "Design for Wearability," in Wearable Computers, 1998. Digest of Papers. Second International Symposium on, vol., no., pp.116-122, 19-20 Oct. 1998, doi: 
0.1109/ISWC.1998.729537,

http://ieeexplore.ieee.org/stamp/stamp.jsp?tp=\&arnumber=729537\&isnumber=15725

1.13. M. Stoppa and A. Chiolerio, "Wearable Electronics and Smart Textiles: A Critical Review," Sensors, vol. 14, no. 7, pp. 11957-11992, Jul. 2014.

1.14. V. G. Motti and K. Caine, "Human Factors Considerations in the Design of Wearable Devices," Proceedings of the Human Factors and Ergonomics Society 58th Annual Meeting, pp. 1821-1824, 2014

1.15. G. Cho, "Smart Clothing Technology and Applications (Human Factors and Ergonomics)," CRC Press, pp. 11, 2010

1.16. D. Heussner, "Wearable Technologies Present Packaging Challenges," [Online]. [Accessed: 12-February 2017]. Available from: http://electronicdesign.com/digitalics/wearable-technologies-present-packaging-challenges

1.17. Wicking Fabric [Online]. [Accessed 12-February-2017]. Available from: http://www.olorun-sports.com/blogs/news/49166979-wicking-fabric

1.18. Apparel Fit Guide [Online]. [Accessed 12-February-2017]. Available from: https://ripleaughtdesign.com/support/fit-guide/

1.19. R. Daws, "Future wearables use your body to communicate" [Online]. [Accessed 12February-2017]. Available from: http://www.wearabletechnologynews.com/news/2015/sep/03/future-wearables-use-your-body-communicate/

1.20. D. Cottet, J. Grzyb, T. Kirstein and G. Tröster, "Electrical Characterisation of Textile Transmission Lines," IEEE Transaction on Advance Packaging, Vol. 26, N0. 2, pp. 182-190, May 2003.

1.21. T. Kirstein, D. Cottet, J. Grzyb and G. Tröster, "Textiles for Signal Transmission in Wearables," Proceedings Workshop on Modelling, Analysis and Middleware Support for Electronic Textiles MAMSET, San Jose, CA, 6, pp. 9-14, October 2002.

1.22. J. R. Wait, "Electromagnetic Theory of the Loosely Braided Coaxial Cable: Part I," in IEEE Transactions on Microwave Theory and Techniques, vol. 24, no. 9, pp. 547553, September 1976

1.23. D. A. Hill, "Electromagnetic Theory of the Loosely Braided Coaxial Cable: Part IINumerical Results," in IEEE Transaction on Microwave Theory and Techniques, vol. MTT-28, no. 4, pp. 326-331, April 1980

1.24. D. A. Hill and J. R. Wail, "Propagation Along a Coaxial Cable with a Helical Shield," in IEEE Transactions on Microwave Theory and Techniques, vol. MTT-28, no. 2, pp. 84-89, February 1980 


\section{Chapter 2}

\section{Theoretical Background on Transmission Lines}

Wearable transmission lines are required to have good electrical characteristics such as low D.C. resistance and low reflection and resistive losses at radio frequencies when used for transmitting signals between two or more wearable devices. The theory of transmission lines is significant in understanding the behavior of wearable transmission lines. This chapter is aimed at reviewing properties of electrical transmission lines and transmission line losses. Methods of implementing wearable transmission lines and a description of the conductive thread used in the construction of the stitched transmission line are also presented.

\subsection{Introduction}

Generally, waves can be propagated in unbounded (unguided) or bounded (guided) media. In unguided structures, the wave propagates throughout space and the EM energy associated with it spreads over a wide area. However, a guided structure serves to direct the propagation of energy from its source to the load. Typical examples of these guided structures are transmission lines and waveguides. Transmission lines are used for purposes such as connecting radio transmitters and receivers with their antenna elements, connections between computers in a network or between a hydroelectric generating plant and a substation some hundreds of miles afar, interconnects between stereo systems, connection between a cable service provider and a TV set, and connections between devices on a circuit board designed to operate at high frequencies [2.1]-[2.2].

Essentially a transmission line is a two-port network, with each port consisting of two terminals (see Fig.2.1), with one of the ports connected to a source or a generator and the other connected to the receiving end, which could be any circuit generating an output voltage, such as a radar transmitter, an amplifier, or a computer terminal operating in transmission mode. Transmission line types include parallel line (ladder line, twisted pair), coaxial cable, Stripline, and microstrip. 


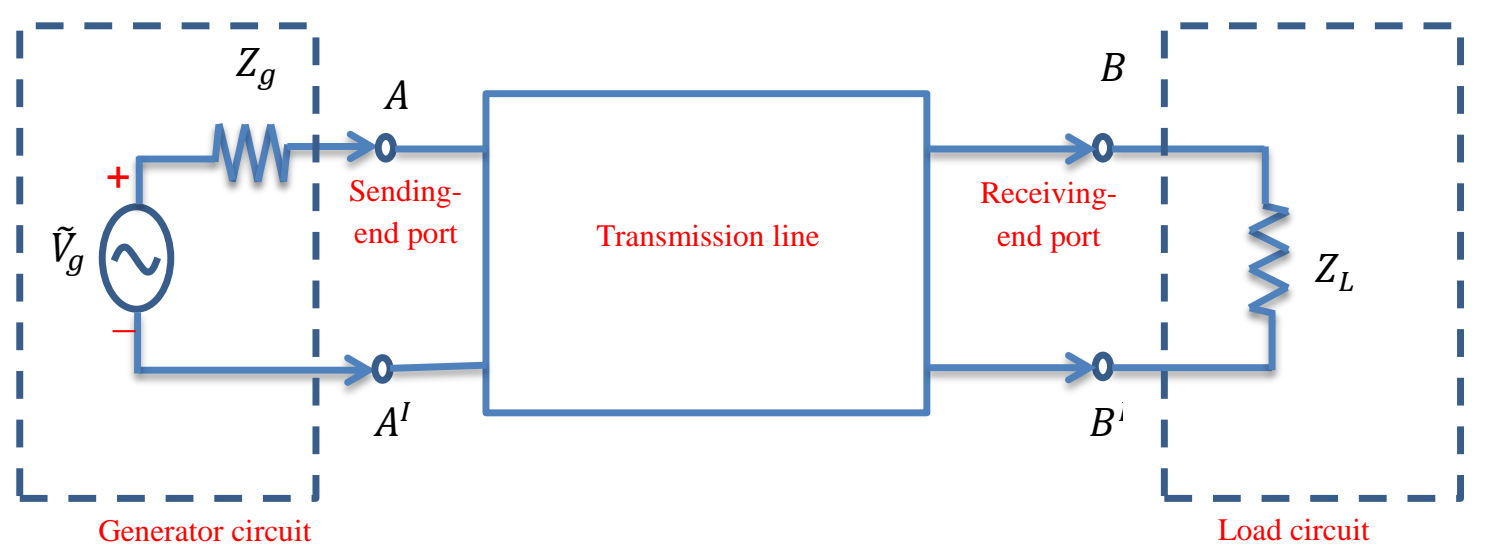

Figure 2.1 Transmission line as a two-port network [2.3]

Transmission lines can be further classified as follows:

a) Transverse electromagnetic (TEM) modes: neither electric nor magnetic field in the direction of propagation

b) Transverse electric (TE) modes: no electric field in the direction of propagation. These are sometimes called $\mathrm{H}$ modes because there is only a magnetic field along the direction of propagation ( $\mathrm{H}$ is the conventional symbol for magnetic field).

c) Transverse magnetic (TM) modes: no magnetic field in the direction of propagation. These are sometimes called E-modes because there is only an electric field along the direction of propagation

d) Hybrid modes: resulting from a combination of TE and TM modes with non-zero electric and magnetic fields in the direction of propagation.

The TEM mode is the dominant mode and has no cut-off frequency. Thus it propagates all the way to DC. While the TE, TM and Hybrid modes are higher order modes which can exist if the operating frequency is high enough and the boundary conditions for these modes to exist are met. Usually, all higher-order modes have different cut-off frequencies, and they start propagating just above their cut-off frequency. The cut-off frequency is given as

$f_{c}=\frac{c}{\lambda_{c}}=\frac{c}{\pi\left(\frac{D_{0}+D_{b}}{2}\right) \sqrt{\mu_{r} \varepsilon_{r}}}$ 
Where the outer diameter of the dielectric is $D_{0}, D_{b}$ is the diameter of the metal braid shield and $\lambda_{c}$ is the cut-off wavelength, with $\lambda_{c}$ given as

$\lambda_{c}=\pi\left(\frac{D_{0}+D_{b}}{2}\right) \sqrt{\mu_{r} \varepsilon_{r}}$

For $\mu_{r}=1, D_{0}$ and $D_{b}$ in $m m$,

$f_{c}(G H z)=\frac{190.85}{\left(D_{0}+D_{b}\right) \sqrt{\varepsilon_{r}}}$

\section{[2.3]- [2.5]}

The TEM mode is considered in this thesis.

\subsection{Transmission Line Parameters}

Essentially a transmission line has four line parameters namely; capacitance per unit length, $\boldsymbol{C}$, conductance per unit length, $\boldsymbol{G}$, resistance per unit length, $\boldsymbol{R}$, and inductance per unit length, $\boldsymbol{L}$. These line parameters are not discrete or lumped but distributed along the line. Table 2-2 provides a summary of the three common transmission line types, with their geometric dimensions as depicted in Fig.2-2, 2-3 and 2-4.

The stitched wearable transmission line can be considered as a two-wire transmission line. A number of known methods of implementing two wire transmission lines can be found in [2.6].

Table 2.1 Transmission line parameters for three line types [2.6]

\begin{tabular}{ccccc}
\hline Parameter & Twin-Wire Line & Coaxial Line & Parallel-Plate Line & Unit \\
\hline $\boldsymbol{R}$ & $\frac{1}{\pi \mathrm{r} \sigma_{\text {cond. }} \delta}$ & $\frac{1}{2 \pi \sigma_{\text {cond. }} \delta}\left(\frac{1}{a}+\frac{1}{b}\right)$ & $\frac{2}{w \sigma_{\text {cond. }} \delta}$ & $\Omega / m$ \\
$\boldsymbol{L}$ & $\frac{\mu}{\pi} \operatorname{acosh}\left(\frac{D}{2 a}\right)$ & $\frac{\mu}{2 \pi} \ln \left(\frac{b}{a}\right)$ & $\mu \frac{d}{w}$ & $H / m$ \\
$\boldsymbol{G}$ & $\frac{\pi \sigma_{\text {diel. }}}{\operatorname{acosh}\left(\frac{D}{2 a}\right)}$ & $\frac{2 \pi \sigma_{\text {diel. }}}{\ln \left(\frac{b}{a}\right)}$ & $\sigma_{\text {diel. }} \frac{w}{d}$ & $S / m$ \\
& $\frac{\pi \varepsilon}{\operatorname{Cosh}\left(\frac{D}{2 a}\right)}$ & $\frac{2 \pi \varepsilon}{\ln \left(\frac{b}{a}\right)}$ & $\varepsilon \frac{w}{d}$ & $F / m$ \\
\hline
\end{tabular}


Where $\pi, \mathrm{a}, b, w, \mu, D, d, \sigma_{\text {diel. }}, \sigma_{\text {cond. }}, \delta, \varepsilon, \boldsymbol{R}, \boldsymbol{L}, \boldsymbol{G}$ and $\boldsymbol{C}$ are as defined in the List of Symbols (See Page VII)

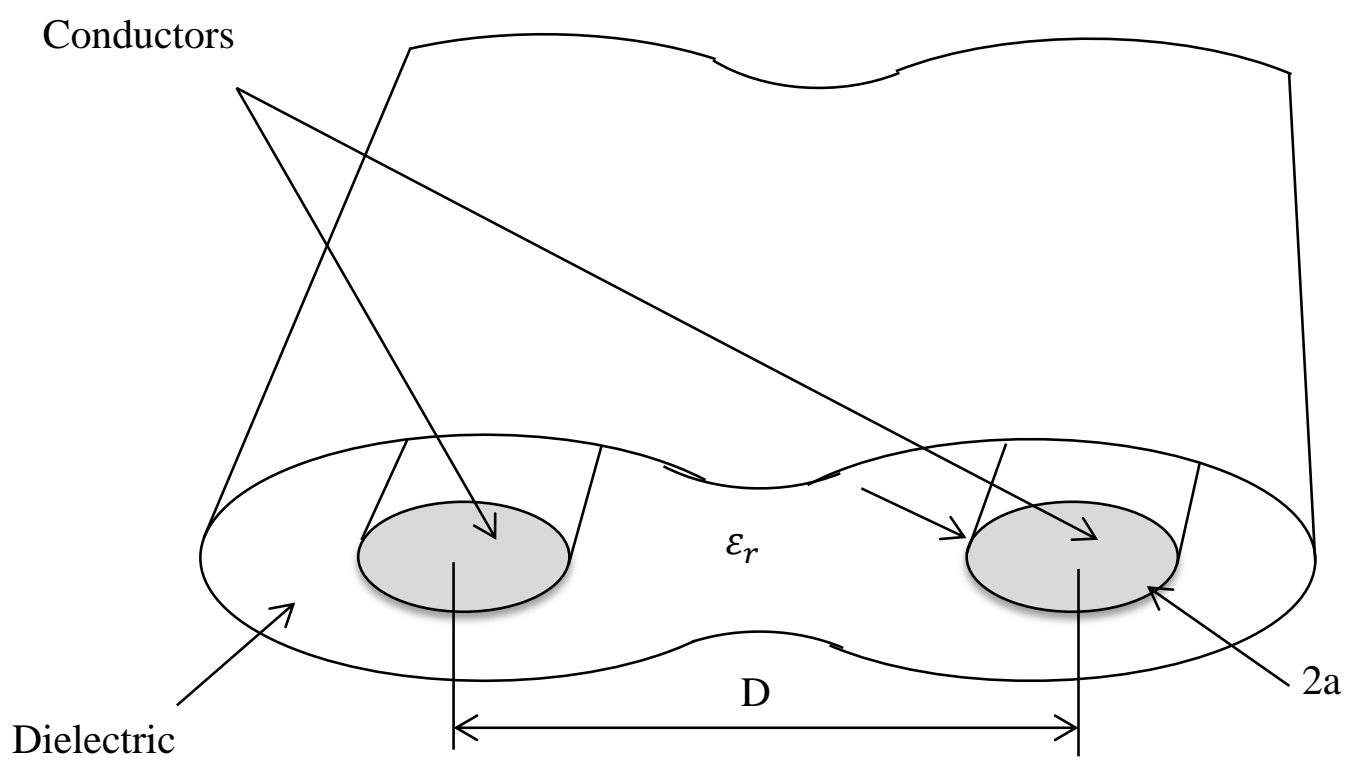

Figure 2.2 Twin-wire parallel conductor transmission line 


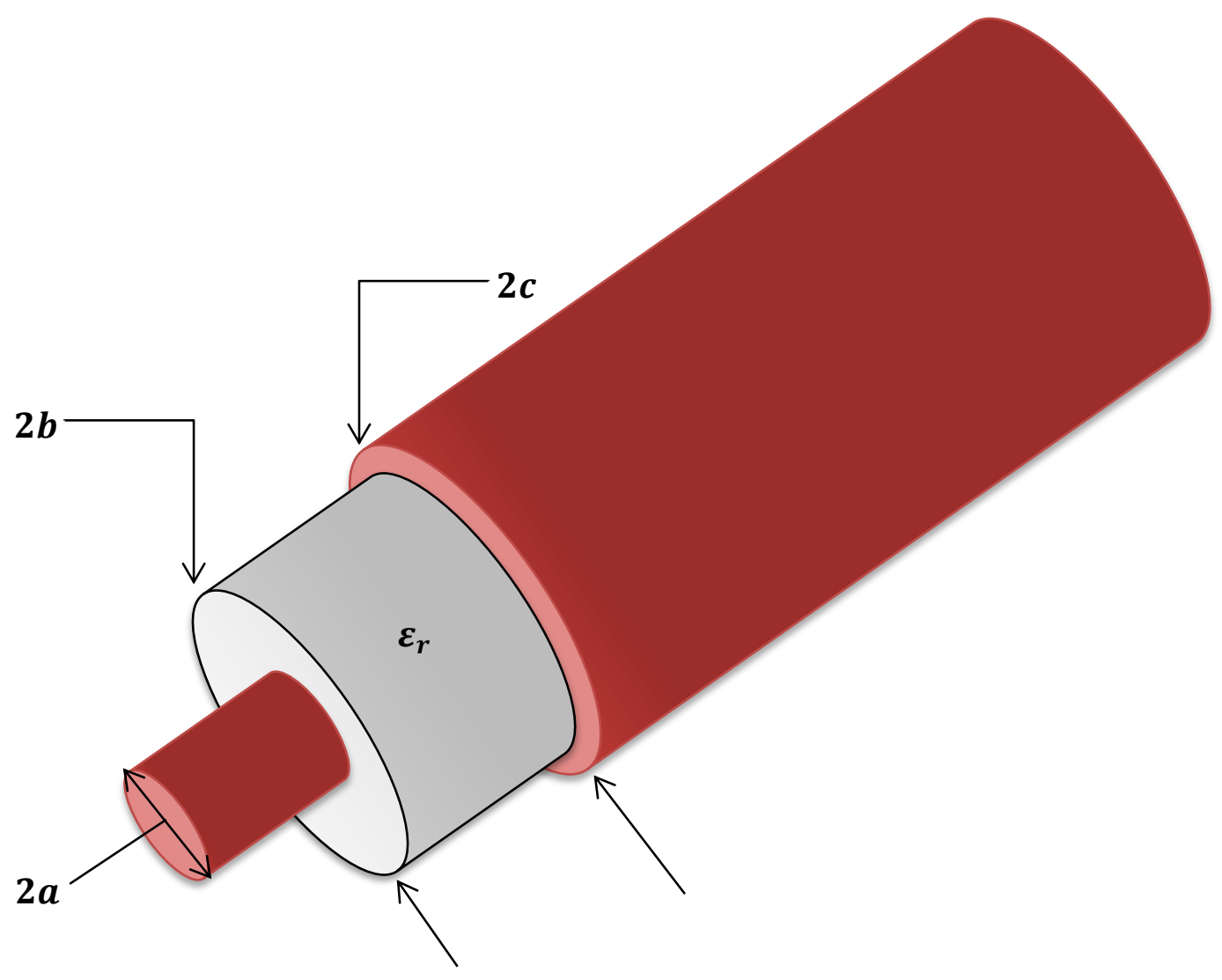

Figure 2.3 Coaxial cable transmission line

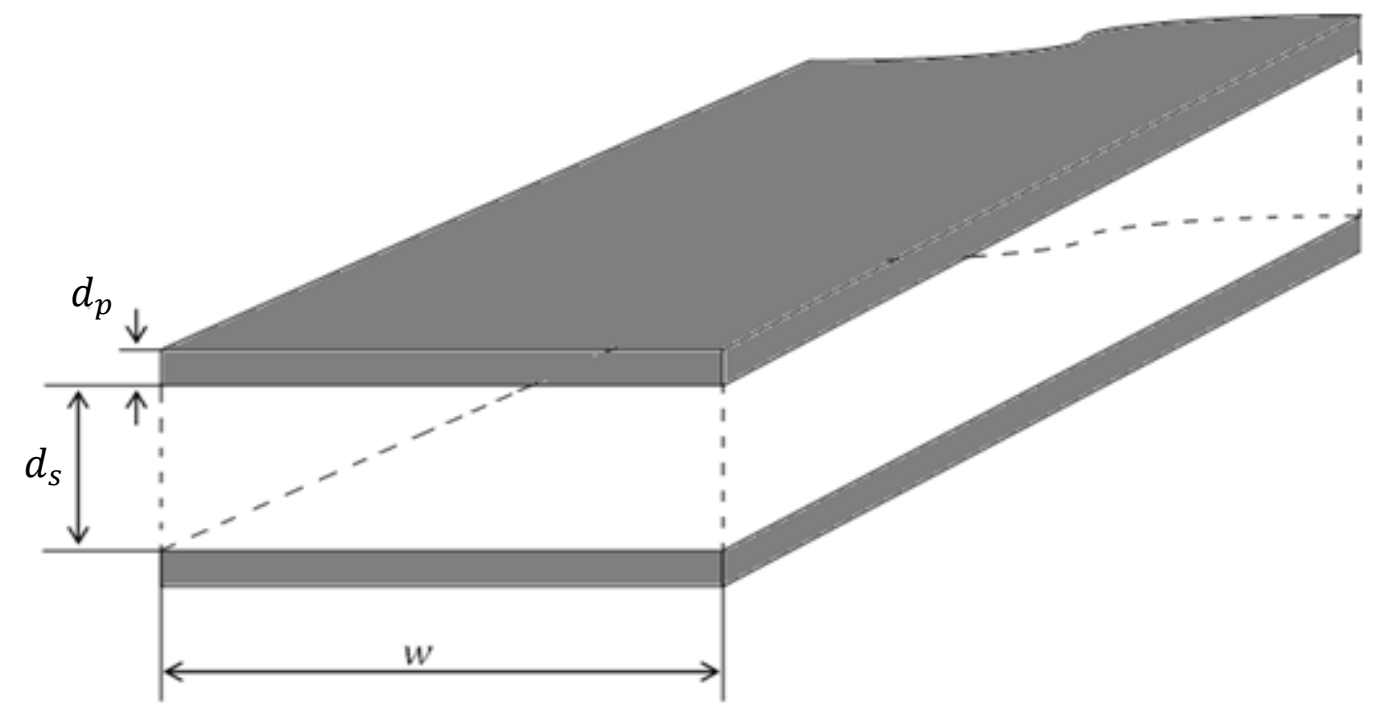

Figure 2.4 Parallel plate transmission line; plate width w is large compared with the separation $d_{s}$ 


\subsection{Transmission line Equations}

Consider a two-wire transmission line which supports a TEM-wave, where the electric and magnetic fields $\vec{E}$ and $\vec{H}$ are related to the voltage $V$ and current $I$ as follows:

$V=-\int \vec{E} \cdot d I$

$I=\oint \vec{H} . d I$

Circuit quantities $V$ and $I$ will be to solve the transmission line problem instead of solving the field quantities $\vec{E}$ and $\vec{H}$.

\section{Lossy Transmission Line}

Here the conductors that make up the line are imperfect $\left(\sigma_{c} \neq \infty\right)$ and the dielectric material, in which the conductors are embedded are lossy $\sigma \neq 0$. The resistance and inductance of the conductor are represented by a series resistor, $\boldsymbol{R}$, and inductor, $\boldsymbol{L}$, while the capacitance between the two conductors is represented by a shunt capacitor, $\boldsymbol{C}$, and the conductance of the dielectric material separating the two conductors by a conductance, $\boldsymbol{G}$, shunted between the signal wire and the return wire.

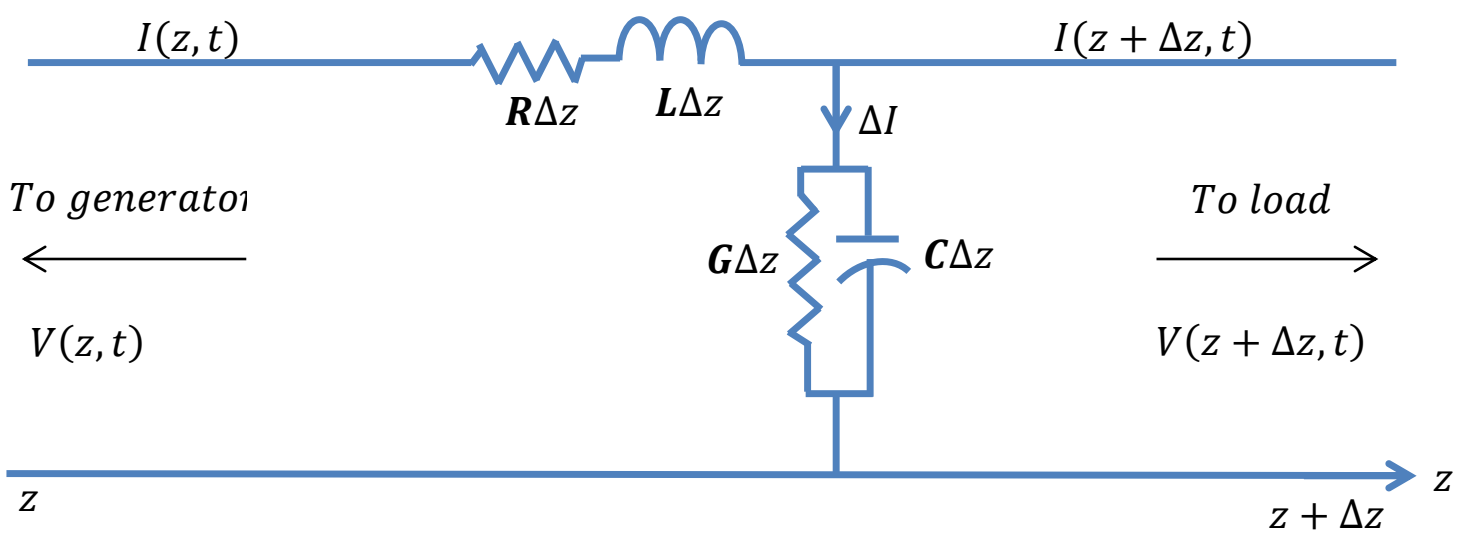

Figure 2.5 Equivalent circuit model of a lossy transmission line [2.1] 
Applying Kirchhoff's voltage law to the outer loop of Fig.2.5,

$V(z, t)=\boldsymbol{R} \Delta z I(z, t)+\boldsymbol{L} \Delta z \frac{\partial I(z, t)}{\partial t}+V(z+\Delta z, t)$

Or

$-\frac{V(z+\Delta z, t)-V(z, t)}{\Delta z}=\boldsymbol{R} I(z, t)+\boldsymbol{L} \frac{\partial I(z, t)}{\partial t}$

As $\Delta z \rightarrow 0$, eq. (2.6) becomes

$-\frac{\partial V(z, t)}{\partial z}=\boldsymbol{R} I(z, t)+\boldsymbol{L} \frac{\partial I(z, t)}{\partial t}$

Equally, applying Kirchhoff's current law to the main node of the circuit in Fig.2.5 gives

$I(z, t)=I(z+\Delta z, t)+\Delta I$

$I(z, t)=I(z+\Delta z, t)+\boldsymbol{G} \Delta z V(z+\Delta z, t)+\boldsymbol{C} \Delta z \frac{\partial V(z+\Delta z, t)}{\partial t}$

Or

$\frac{I(z+\Delta z, t)-I(z, t)}{\Delta z}=\boldsymbol{G} V(z+\Delta z, t)+\boldsymbol{C} \frac{\partial V(z+\Delta z, t)}{\partial t}$

As $\Delta z \rightarrow 0$, eq. (2.8) becomes

$-\frac{\partial I(z, t)}{\partial z}=\boldsymbol{G} V(z, t)+\boldsymbol{C} \frac{\partial V(z, t)}{\partial t}$

If we assume harmonic time dependence, so that

$V(z, t)=\operatorname{Re}\left[V_{s}(z) e^{j \omega t}\right]$

$I(z, t)=\operatorname{Re}\left[I_{s}(z) e^{j \omega t}\right]$

Where $V_{S}(z)$ and $I_{S}(z)$ are the phasor forms of $V(z, t)$ and $I(z, t)$ respectively, eqs.2.7 and 2.9 become

$-\frac{d V_{s}}{d z}=(\boldsymbol{R}+j \omega \boldsymbol{L}) I_{S}$

$-\frac{d I_{s}}{d z}=(\boldsymbol{G}+j \omega \boldsymbol{C}) V_{s}$

Taking the second derivative of $V_{s}$ Eq.2.11 and using Eq.2.12, we obtain 
$\frac{d^{2} V_{s}}{d z^{2}}=(\boldsymbol{R}+j \omega \boldsymbol{L})(\boldsymbol{G}+j \omega \boldsymbol{C}) V_{s}$

$\frac{d^{2} V_{s}}{d z^{2}}-\gamma^{2} V_{s}=0$

Where

$\gamma=\alpha+j \beta=\sqrt{(\boldsymbol{R}+j \omega \boldsymbol{L})(\boldsymbol{G}+j \omega \boldsymbol{C})}$

Similarly by taking the second derivative of $I_{s}$ in Eq. (2.12) and using Eq. (2.11), we obtain

$\frac{d^{2} I_{S}}{d z^{2}}-\gamma^{2} I_{s}=0$

Hence $\gamma$ in Eq. (2.13) and (2.15) is the propagation constant (in per meter), $\alpha$ is the attenuation constant (in nepers per meter or decibels ${ }^{2}$ per meter), and $\beta$ is the phase constant (in radians per meter). The wavelength $\lambda$ and wave velocity, $u$ are, respectively, given by

$\lambda=\frac{2 \pi}{\beta}$

$u=\frac{\omega}{\beta}=f \lambda$

The solutions of the linear homogeneous differential Eq. (2.13) and (2.15) are given by

$V_{s}(z)=V_{0}^{+} e^{-\gamma z}+V_{0}^{-} e^{\gamma z}$

And

$I_{S}(z)=I_{0}^{+} e^{-\gamma z}+I_{0}^{-} e^{\gamma z}$

Where $V_{0}^{+}, V_{0}^{-}, I_{0}^{+}$and $I_{0}^{-}$are wave amplitudes with the + and - signs denoting wave traveling along $+z-$ and -z-directions respectively. Thus, we obtain the instantaneous expression for voltage as

$V(z, t)=\operatorname{Re}\left[V_{s}(z) e^{-j \omega t}\right]$

$V(z, t)=V_{0}^{+} e^{-\alpha z} \cos (\omega t-\beta z)+V_{0}^{-} e^{\alpha z} \cos (\omega t+\beta z)$

The characteristic impedance $Z_{0}$ of the line is the ratio of positively travelling voltage to the current wave at any point on the line. It is akin to the intrinsic impedance of the medium of wave propagation $\eta$. 
$Z_{0}=\frac{V_{0}^{+}}{I_{0}^{+}}=-\frac{V_{0}^{-}}{I_{0}^{-}}=\frac{\boldsymbol{R}+j \omega \boldsymbol{L}}{\gamma}=\frac{\gamma}{G+j \omega \boldsymbol{C}}$

$Z_{0}=\sqrt{\frac{R+j \omega L}{G+j \omega \boldsymbol{C}}}=R_{0}+j X_{0}$

Where $R_{0}$ and $X_{0}$ are the real and imaginary parts of $Z_{0}$ [2.1].

\section{Lossless Transmission}

For an ideal lossless transmission line, the conductors of the line are perfect, $\sigma_{c} \approx \infty$ and the dielectric medium separating them is lossless $\sigma \cong 0$. Hence

$\boldsymbol{R}=\boldsymbol{G}=0$

The inductance of a length $(d x)$ of the conductor is represented by a series inductor, $\boldsymbol{L} d x$, while the capacitance between a length $(d x)$ of the two conductors is represented by a shunt capacitor, $\boldsymbol{C} d x$.

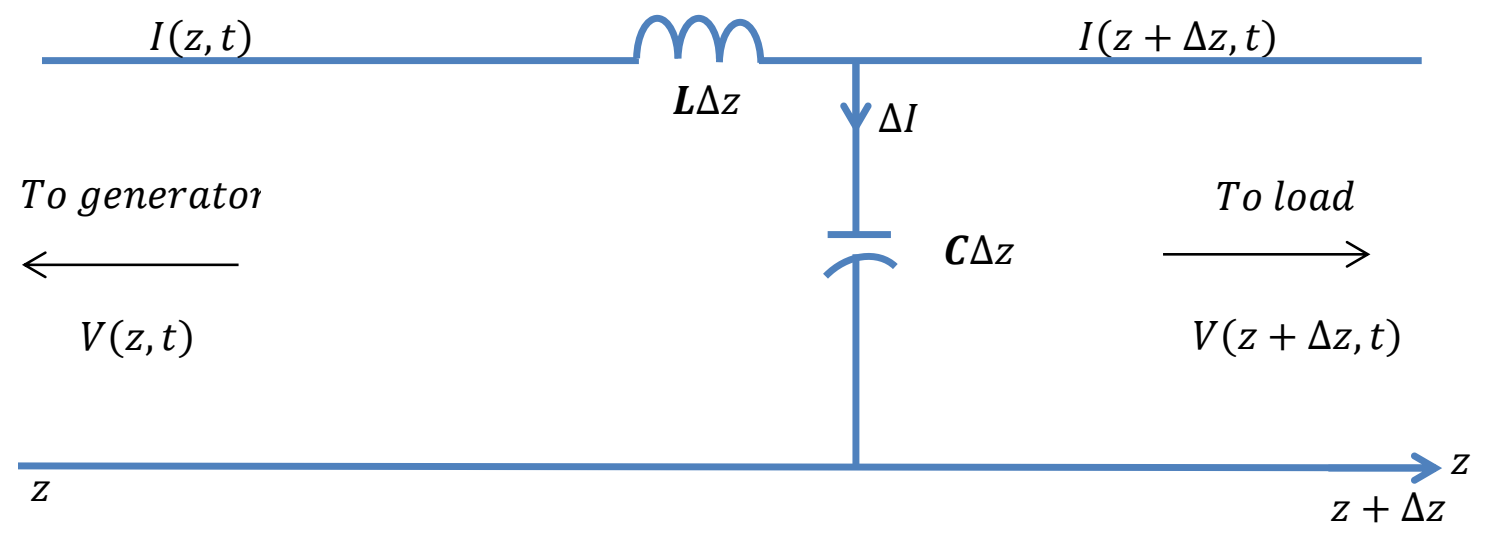

Figure 2.6 Equivalent circuit model of a lossless transmission line [2.1]

Consequently Eq. (2.14), (2.14) and (2.22) becomes

$\alpha=0, \quad \gamma=j \beta=j \omega \sqrt{\boldsymbol{L C}}$ 
$u=\frac{\omega}{\beta}=\frac{1}{\sqrt{L C}}=f \lambda$

$X_{0}=0, \quad Z_{0}=R_{0}=\sqrt{\frac{L}{C}}$

[2.1]

\subsection{Input Impedance, Voltage Standing Wave Ratio, and Power}

\section{Input Impedance}

Consider a transmission line of length $l$, characterised by $\gamma$ and $Z_{0}$, connected to a load $Z_{L}$ as shown in Fig.2.7. Looking into the line, the generator sees the line with the load as input impedance $Z_{\text {in }}$.

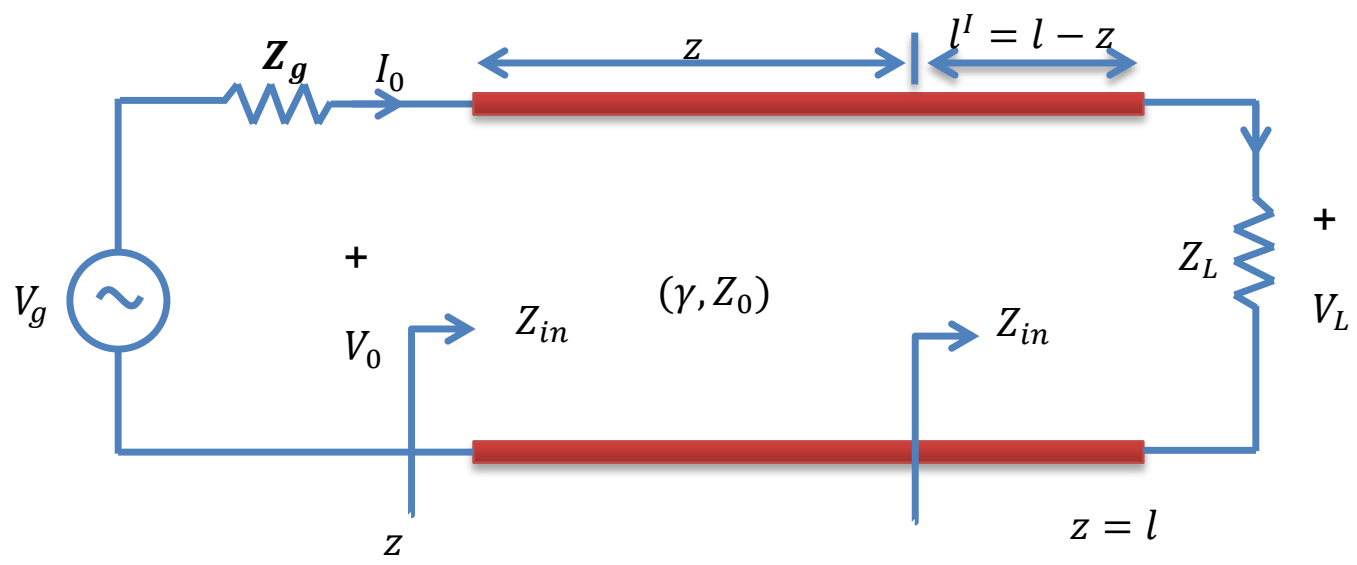

(a)

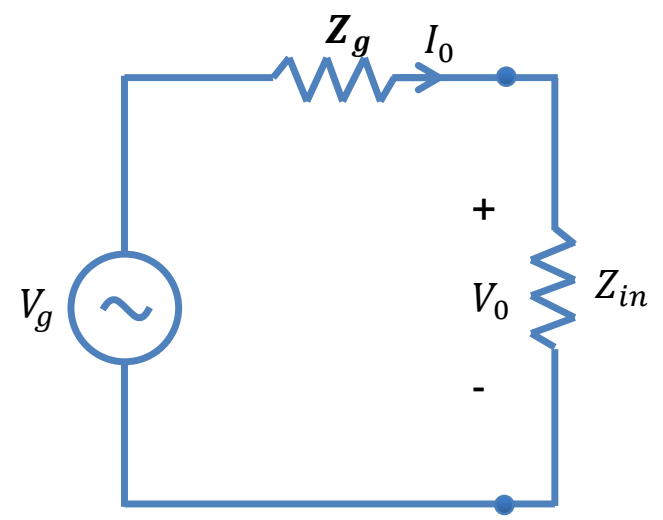

(b)

Figure 2.7(a) Input impedance due to a line terminated by a load; (b) equivalent circuit for finding $\boldsymbol{V}_{\mathbf{0}}$ and $\boldsymbol{I}_{\mathbf{0}}$ in terms of $\boldsymbol{Z}_{\boldsymbol{i n}}$ at the input 
Suppose the line is extended from $z=0$ at the generator to $z=l$ at the load and using Eq. (2.18) and (2.19), where Eq. (2.21) has been incorporated, to find $V_{0}^{+}$and $V_{0}^{-}$, the terminal conditions must be given.

$$
\begin{aligned}
& V_{s}(z)=V_{0}^{+} e^{-\gamma z}+V_{0}^{-} e^{\gamma z} \\
& I_{S}(z)=\frac{V_{0}^{+}}{Z_{0}} e^{-\gamma z}-\frac{V_{0}^{-}}{z_{0}} e^{\gamma z}
\end{aligned}
$$

Suppose the given conditions at the input are

$V_{0}=V(z=0), \quad I_{0}=I(z=0)$

Putting these into Eq. (2.25) and (2.26) yields

$$
\begin{aligned}
& V_{0}^{+}=\frac{1}{2}\left(V_{0}+Z_{0} I_{0}\right) \\
& V_{0}^{-}=\frac{1}{2}\left(V_{0}-Z_{0} I_{0}\right)
\end{aligned}
$$

If the input impedance at the terminals is $Z_{i n}$, the input voltage $V_{0}$ and the input current $I_{0}$ are obtained from Fig.2.7 (b) as

$$
V_{0}=\frac{Z_{\text {in }}}{Z_{\text {in }}+Z_{g}} V_{g}, \quad I_{0}=\frac{V_{g}}{Z_{\text {in }}+Z_{g}}
$$

Conversely, if conditions at the load are

$V_{L}=V(z=l), \quad I_{L}=I(z=l)$

Putting these into Eq. (2.25) and (2.26) yields

$$
\begin{aligned}
& V_{0}^{+}=\frac{1}{2}\left(V_{L}+Z_{0} I_{L}\right) e^{\gamma l} \\
& V_{0}^{-}=\frac{1}{2}\left(V_{L}-Z_{0} I_{L}\right) e^{-\gamma l}
\end{aligned}
$$

To determine the input impedance $Z_{\text {in }}$ at any point on the line, we use Eq. (2.25) and (2.26)

$$
Z_{\text {in }}=\frac{V_{S}(z)}{I_{S}(z)}=\frac{Z_{0}\left(V_{0}^{+}+V_{0}^{-}\right)}{\left(V_{0}^{+}-V_{0}^{-}\right)}
$$

Putting Eq. (2.31) into (2.32) and using

$$
\cosh \gamma l=\frac{e^{\gamma l}+e^{-\gamma l}}{2}, \quad \sinh \gamma l=\frac{e^{\gamma l}-e^{-\gamma l}}{2}
$$

Or 
$\tanh \gamma l=\frac{\sinh \gamma l}{\cosh \gamma l}=\frac{e^{\gamma l}-e^{-\gamma l}}{e^{\gamma l}+e^{-\gamma l}}$

$Z_{\text {in }}$ is given by,

$Z_{\text {in }}=Z_{0}\left[\frac{Z_{L}+Z_{0} \tanh \gamma l}{Z_{0}+Z_{L} \tanh \gamma l}\right] \quad$ (Lossy)

To find the $Z_{\text {in }}$ at a distance $l^{I}$ from the load as in Fig.2.7(a), $l$ is replaced with $l^{I}$.

For a lossless line, $\gamma=j \beta, \tanh j \beta l=j \tanh \beta l$, and $Z_{0}=R_{0}$, so that eq.(2.34) now becomes

$Z_{\text {in }}=Z_{0}\left[\frac{Z_{L}+j Z_{0} \tanh \beta l}{Z_{0}+j Z_{L} \tanh \beta l}\right] \quad$ (Lossless)

This shows that the input impedance varies periodically with distance $l$ from the load. $\beta l$ in Eq. (2.35) is usually referred as the electrical length of the line expressed in degrees or radians [2.1].

\section{Voltage Standing Wave Ratio (VSWR)}

Voltage reflection coefficient at the load, $\Gamma_{L}$, is the ratio of the voltage reflection wave to the incident wave at the load. That is,

$\Gamma_{L}=\frac{V_{0}^{-} e^{\gamma l}}{V_{0}^{+} e^{-\gamma l}}$

Putting $V_{0}^{-}$and $V_{0}^{+}$in Eq. (2.31) into Eq. (2.36) and incorporating $V_{L}=Z_{L} / I_{L}$ yields

$\Gamma_{L}=\frac{Z_{L}-Z_{0}}{Z_{L}+Z_{0}}$

Furthermore, the voltage reflection coefficient at any point on the line is the ratio of the magnitude of the reflected voltage wave to that of the incident wave. That is,

$$
\Gamma(z)=\frac{V_{0}^{-} e^{\gamma l}}{V_{0}^{+} e^{-\gamma l}}=\frac{V_{0}^{-}}{V_{0}^{+}} e^{2 \gamma z}
$$

But $z=l-l^{I}$; Substituting and combining with Eq. (2.36) yields

$\Gamma(z)=\frac{V_{0}^{-}}{V_{0}^{+}} e^{2 \gamma l} e^{-2 \gamma l^{I}}=\Gamma_{L} e^{-2 \gamma l^{I}}$ 
The current reflection at any point on the line is negative of the voltage reflection at that point.

Thus the current reflection coefficient at the load is given by

$$
-\Gamma_{L}=\frac{I_{0}^{-} e^{\gamma l}}{I_{0}^{+} e^{-\gamma l}}
$$

Hence the standing wave ratio $s$, (denoted as SWR) is defined as

$S=\frac{V_{\max }}{V_{\min }}=\frac{I_{\max }}{I_{\min }}=\frac{1+\left|\Gamma_{L}\right|}{1-\left|\Gamma_{L}\right|}$

It can be easily shown that; $I_{\max }=\frac{V_{\max }}{Z_{0}}$ and $I_{\min }=\frac{V_{\min }}{Z_{0}}$. The input impedance $Z_{\text {in }}$ in Eq. (2.35) has a maxima and minima that occur at the maxima and minima of the voltage and current standing wave respectively. It can also be shown that

$\left|Z_{\text {in }}\right|_{\max }=\frac{V_{\max }}{I_{\min }}=s Z_{0}$

And

$\left|Z_{\text {in }}\right|_{\min }=\frac{V_{\min }}{I_{\max }}=\frac{Z_{0}}{s}$

\section{Power}

Transmission lines are a means of transmitting information or power from the source to the load. The average input power at a distance $l$ from the load is given by

$P_{\text {ave }}=\frac{1}{2} \operatorname{Re}\left[V_{s}(l) I_{s}^{*}(l)\right]$

Assuming a lossless line, Eq. (2.25) and (2.26) is substituted in the above equation to obtain

$$
\begin{gathered}
P_{\text {ave }}=\frac{1}{2} \operatorname{Re}\left[V_{0}^{+}\left(e^{j \beta l}+\Gamma e^{-j \beta l}\right) \frac{V_{0}^{+*}}{Z_{0}}\left(e^{-j \beta l}-\Gamma^{*} e^{j \beta l}\right)\right] \\
P_{\text {ave }}=\frac{1}{2} \operatorname{Re}\left[\frac{\left|V_{0}^{+}\right|^{2}}{Z_{0}}\left(1-|\Gamma|^{2}+\Gamma e^{-2 j \beta l}-\Gamma^{*} e^{2 j \beta l}\right)\right]
\end{gathered}
$$

Since the last two terms are purely imaginary, we have 
$P_{\text {ave }}=\left[\frac{\left|V_{0}^{+}\right|^{2}}{2 Z_{0}}\left(1-|\Gamma|^{2}\right)\right]$

The first term is the incident power $P_{i}$, while the second term is the reflected power $P_{r}$.

Hence Eq. (2.41) may be written as

$P_{t}=P_{i}-P_{r}$

We note from Eq. (2.41) that the power is constant and does not depend on $l$ since it is a lossless line. Also, the maximum power is delivered to the load when $\Gamma=0$, as expected [2.1].

\section{Special Cases with Shorted, Open-Circuited and Matched Line}

A. Shorted Line $\left(Z_{L}=0\right)$

Here, Eq. (2.35) becomes

$Z_{S c}=\left.Z_{\text {in }}\right|_{Z_{L}=0}=j Z_{0} \tan \beta l$

Also

$\Gamma_{L}=-1, \quad s=\infty$

B. Open-Circuited Line $\left(Z_{L}=\infty\right)$

Here, Eq. (2.35) becomes

$Z_{0 C}=\lim _{Z_{L} \rightarrow \infty} Z_{i n}=\frac{Z_{0}}{j \tan \beta l}=-j Z_{0} \cot \beta l$

And

$\Gamma_{L}=1, \quad s=\infty$

From Eq. (2.44a) and (2.45a),

$Z_{S C} Z_{0 C}=Z_{0}^{2}$

C. Matched Line $\left(Z_{L}=Z_{0}\right)$

Here, Eq. (2.35) becomes

$Z_{\text {in }}=Z_{0}$

And

$\Gamma_{L}=0, \quad s=1$

[2.1] 


\subsection{Transmission Line Loss and Attenuation}

Attenuation is the intrinsic power loss within a coaxial cable. Attenuation is dependent on the coaxial cable design and is also dependent on frequency and length of the coaxial cable. Attenuation in coaxial cables is found to also increase over a period of time due to flexing and inflow of moisture into the coaxial cable. However, it is most affected by DC resistance of the conductor. Conductive threads, when used as transmission lines, tend to have more losses compared to metallic conductors because of their resistance, and these losses tend to increase especially when stitched into apparels and worn by humans as the body also serves as a dielectric medium and the closer the transmission line is to the body the lossier it will be.

All transmission lines have some losses in them and these losses could be a real loss (attenuation) and mismatch loss (rejection). Here the focus is the real loss. The real loss includes conductor loss, radiation and induction loss, and dielectric loss. Transmission line losses are expressed in decibels $(\mathrm{dB})$ while attenuation is expressed in $\mathrm{dB} / 100 \mathrm{ft}$.

\section{A. Conductor loss}

Whenever current flows through the conductors that make up the transmission line, some energy is lost in the form of heat $\left(I^{2} R\right)$ because the resistance of the conductors is never equal to zero. This loss is also referred to as heating loss and is simply power loss. This can be reduced by using a shorter transmission line, using a larger diameter wire or plaiting the line with silver $\left(\sigma=6.30 \times 10^{7} \mathrm{Sm}^{-1}\right.$ at $\left.20^{\circ} \mathrm{C}\right)$ [2.7] which is a better conductor compared to copper $\left(\sigma=5.96 \times 10^{7} \mathrm{Sm}^{-1}\right.$ at $\left.20^{\circ} \mathrm{C}\right)[2.8]$.

Conductor loss also depends somewhat on the frequency due to a phenomenon known as skin effect, which is the tendency of an AC to distribute itself within a conductor so that the current density near the surface of the conductor is greater than that at its core. Skin effect becomes progressively more apparent with increased frequency. To repress this, multi-stranded conductors are used.

\section{B. Radiation and Induction loss}

These are comparable in that both are caused by fields surrounding the conductors. Induction losses occur when the EM field about a conductor cuts through any nearby 
metallic object and current is induced in the object. As a result, power is dissipated in the object and is lost.

Radiation losses occur when magnetic lines of force about a conductor do not return to the conductor when the cycle alternates. These lines of force are projected into space as radiation and this result in power losses. Power is supplied by the source but is not available at the load. Radiation losses depend on the dielectric material, conductor spacing and length of the transmission line. It is also directly proportional to the frequency. This can be minimized by properly shielding the cable.

\section{Dielectric loss}

Dielectric losses are as a result of the heating effect on the dielectric material between the conductors. The difference of potential between two conductors of a metallic transmission line causes dielectric heating. For air transmission line the heating is negligible, while for a solid core transmission line, dielectric heating loss increases with frequency. Dielectric loss is found to be independent of the cable size. As resistive losses increase as the square root of frequency, dielectric losses increase linearly with frequency and hence dominates higher frequencies [2.9]-[2.10].

\subsection{Wearable Transmission Lines}

Wearable transmission lines are transmission lines that form part of the apparels that can be worn by humans and animals. Usually, these are stitched or embedded onto apparels. The textile characteristics of being lightweight, flexible, soft, breathable, robust and washable make them preferable to the conventional transmission lines.

Wearable transmission lines could be power supply lines for electronic circuits or signal lines transmitting signals often with a wavelength that is much greater than the length of the line (low frequency signals) and signal lines that transmit signals with a wavelength that is comparable to the length of the wearable transmission line (high frequency signals). The characterisation of transmission line performance when stitched or embedded onto apparels and worn by humans is also very important in its design. 


\subsubsection{Methods of Implementing Wearable Transmission Lines}

There are many possibilities of implementing a transmission line into a substrate. These include:

a. Direct implementation of conductive transmission lines in the form of conductive wires (also insulated) or electroconductive yarns at the production stage and manufactured in flat textile products such as woven fabrics, knitting, and nonwovens

b. Overprinting an electroconductive medium on a flat textile

c. Spraying or other deposition of an electroconductive medium on a flat textile

d. Incorporating electroconductive paths using sewing or embroidery methods

[2.11]

Examples of these are shown in Fig.2.8, 2.9, 2.10 and 2.11.

In [2.12], a woven conductive fabric with embedded insulated copper wires so as to prevent short-circuits among copper wires was presented for signal transmission. This consists of a polyester multifilament yarn and coated copper wires woven by Sefar Inc. The distance between each metal wire is $500 \mu \mathrm{m}$ in both directions (warp and weft) and the diameter of the copper wire is $40 \mu \mathrm{m}$ and $54 \mu \mathrm{m}$ without and with an insulation coating of $7 \mu \mathrm{m}$ respectively. This was used to integrate simple routing structures into fabrics while utilising standard printed wiring board (PWB) tools. Also, a characterisation of CPW transmission lines printed onto nonwoven textile substrates using conductive inks to determine their suitability for wide-band applications is presented in [2.13]. Here, the polymer thick film (PTF) technique was adopted instead of woven and knitted electronic textiles technique which allows a simple manufacturing process without adding complexities that will increase the cost of production.

With the convenience of using washing machines, humans tend to have the urge to wash their apparels, even when they are not dirty. Hence, the washability of the "wearable transmission line" comes into question. Some approaches have been made to study the washability of wearable transmission lines. In [2.14], the frequency dependent per unit length parameters of a conductive textile transmission line (CCTL) were evaluated through measurements and modeling and a study of its deterioration parameters when subjected to washing cycles. A severe deterioration of the electrical parameters was noticed; however, both experimental and modeling results were seen to be in good agreement within the addressed frequency band. 
Also, the feasibility using digital embroidery and conducting threads to construct transmission lines and potentially antennas was investigated in [2.15]. The threads were examined by assessing their DC resistance at rest while under physical strain and also the RF performance of transmission lines. The RF transmission line measurements validated the DC measurements, which were found to be a reasonable first estimate of RF performance. It was also suggested that the conductivity could also be improved by stitching along the length of (parallel to) the transmission lines and also by using a higher density of stitching. Similarly in [2.16]-[2.17], Didier et al. and Tunde et al. proposed an extensive characterization of textile transmission lines for use in wearable computing applications. The proposed textiles are fabrics with copper fibers in one or two directions and with different yarn fineness, designed in such a way to avoid contact with each other. Measurements of up to $6 \mathrm{GHz}$ from extracted frequency characteristics revealed that the dielectric and ohmic losses do not determine the insertion loss. But the loss is mainly influenced by non-uniform impedance profile along the lines up to the half wavelength and by coupling to parasitic modes above this frequency point.

The use of screen printing technology for transmission lines with controlled impedances on textile was also presented in [2.18]. The proposed transmission line structure revealed a bandwidth of about $4.7 \mathrm{GHz}$ that suits most present-day applications in wearable computing e.g. Bluetooth and USB and a line impedance close to $50 \Omega$, which is a major advantage compared to a two wire-based textile transmission line. The cured silver paste used with a solid content of about $75 \%$ suffered from brittleness. However, the bending of the transmission line through a radius larger than $1 \mathrm{~cm}$ was seen to have no effect on its DC resistance. Conversely, bending with smaller radii or even creasing resulted in an increased resistance due to cracks in the silver paste. This effect was bigger when less printing passes were applied. Ultimately, conductivity was lost as the paste crumbled from the fabric, as such folds and creasing at slightly shifted positions should be avoided. It was also observed that 510 printing passes were also seen to give good results regarding geometrical precision and electrical performance.

The design and characterisation of a differential stretchable transmission line were presented for wearable applications by Jeon et al. in [2.19]. Here a zigzag shaped transmission line designed to allow stretch while maintaining mechanical reliability was embedded in a lowcost Polyurethane thin film which has excellent stretchability and flexibility. While measured results indicate differential mode characteristics impedance of $94 \Omega$ at the normal length which slightly increases as the line stretches due to deformation of the line, the insertion loss 
was seen to slightly reduce as the line stretches. The transmission line can be elongated up to about $25 \%$ guaranteeing electrical performance and mechanical stability compared to the normal state. Also, twisted copper filaments made by yarn covering process were presented for signal and power transmission for electronic textiles in [2.20], while Zhi et al. in [2.21] reported a shielded Stripline formed by silver fabric, conductive thread, foam substrate and conductive glue as a wearable transmission line for broadband operations. The compact and low profile Stripline were seen to possess no dispersion and has high efficiency and low reflection coefficient for frequencies up to $8 \mathrm{GHz}$. The robustness of the design when bent through $90^{\circ}$ and $180^{\circ}$ and tolerances for a wide range of cross-sectional variations and connector offsets were also demonstrated, and it was also shown that the low loss frequency foam substrate material used can be replaced with a low-cost clothing felt without any significant impact on the efficiency.

In this thesis, the idea of a braided coaxial cable is used to implement a stitched transmission line for wearable RF devices through the use of a sewing machine and an improved presser foot. The stitched transmission line is made up of a stripped RG174 braided coaxial cable (whose outer insulating sheet and shield are removed from the cable) which is stitched onto a Denim substrate with the aid of the improved presser foot. This idea presents a wearable transmission line that is easy to install and economical, and with some of the advantages that comes with the use of a braided RG174 coaxial cable such as having a centre signal wire that is made up of a multi-strand twisted copper, which makes it quite flexible and less bulky and also good for tight spaces. 


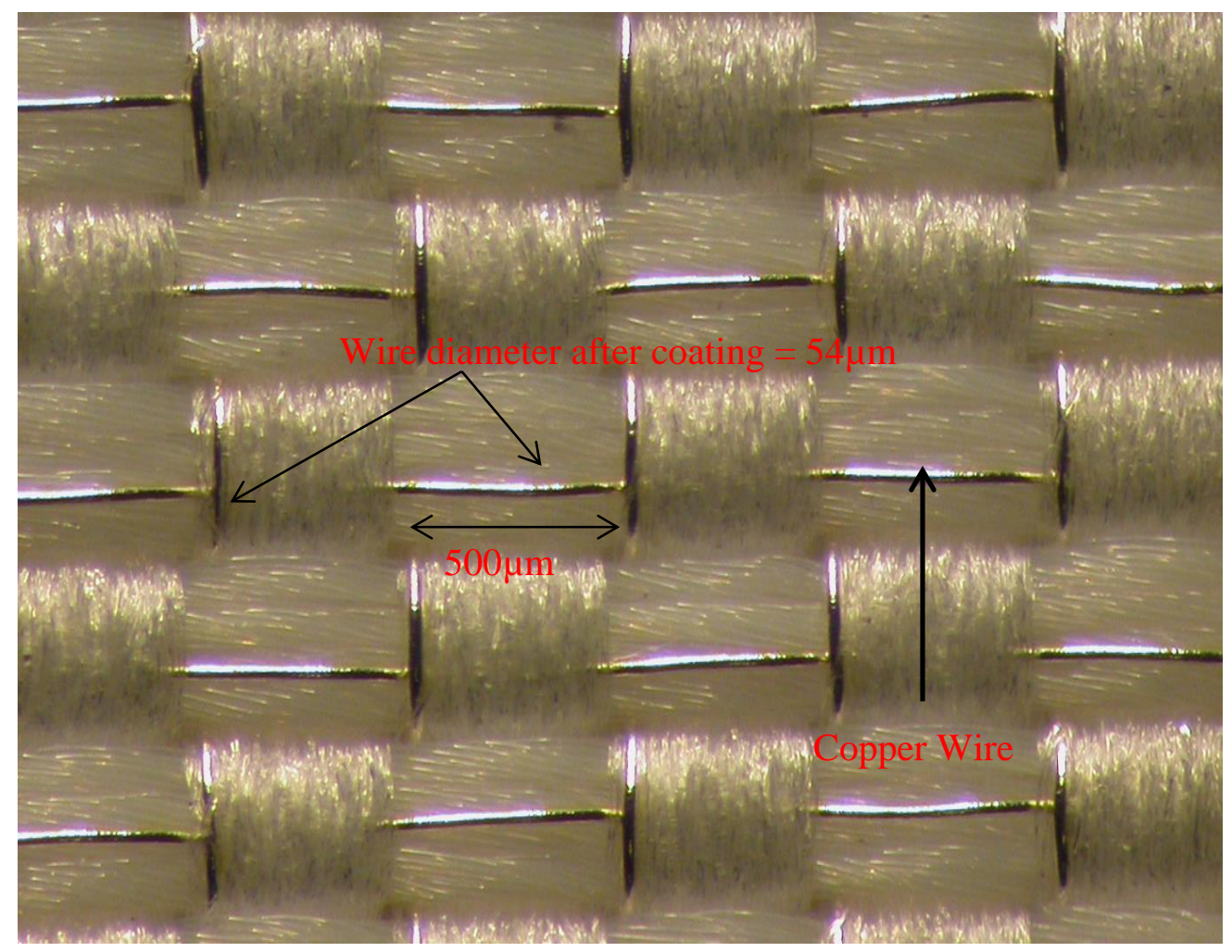

Figure 2.8 Conductive paths made of copper wires in a fabric after Locher et al. [2.12]

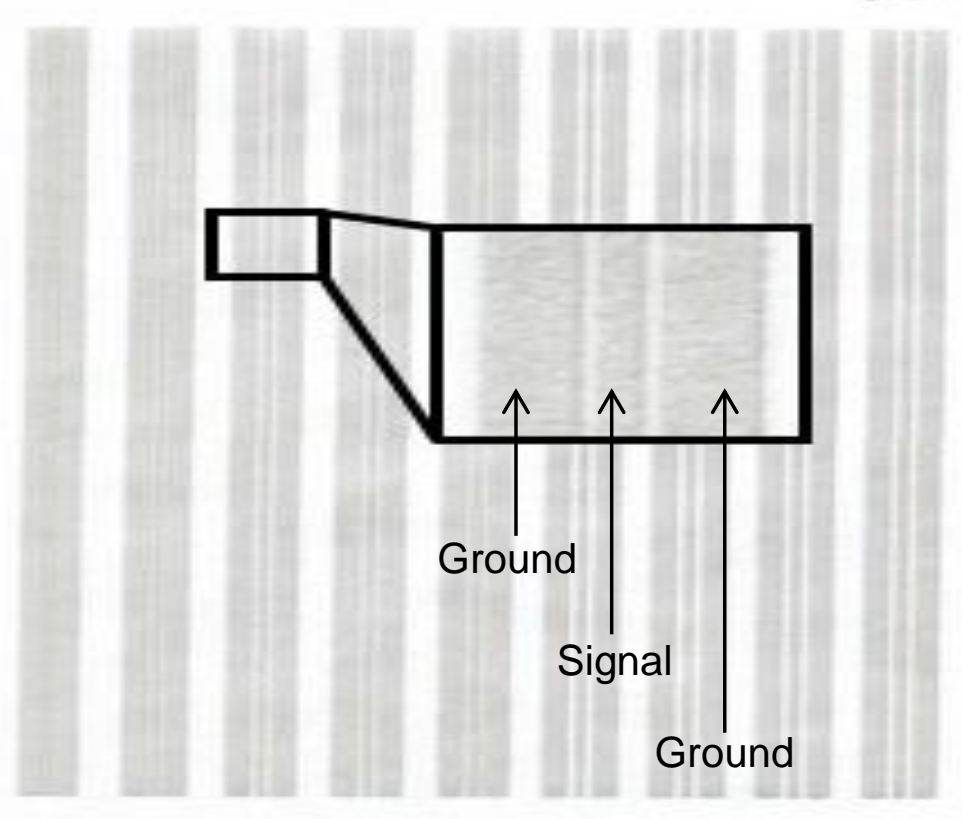

Figure 2.9 Screen Printed Sample of CPWs on Evolon after Merritt et al. [2.13] 


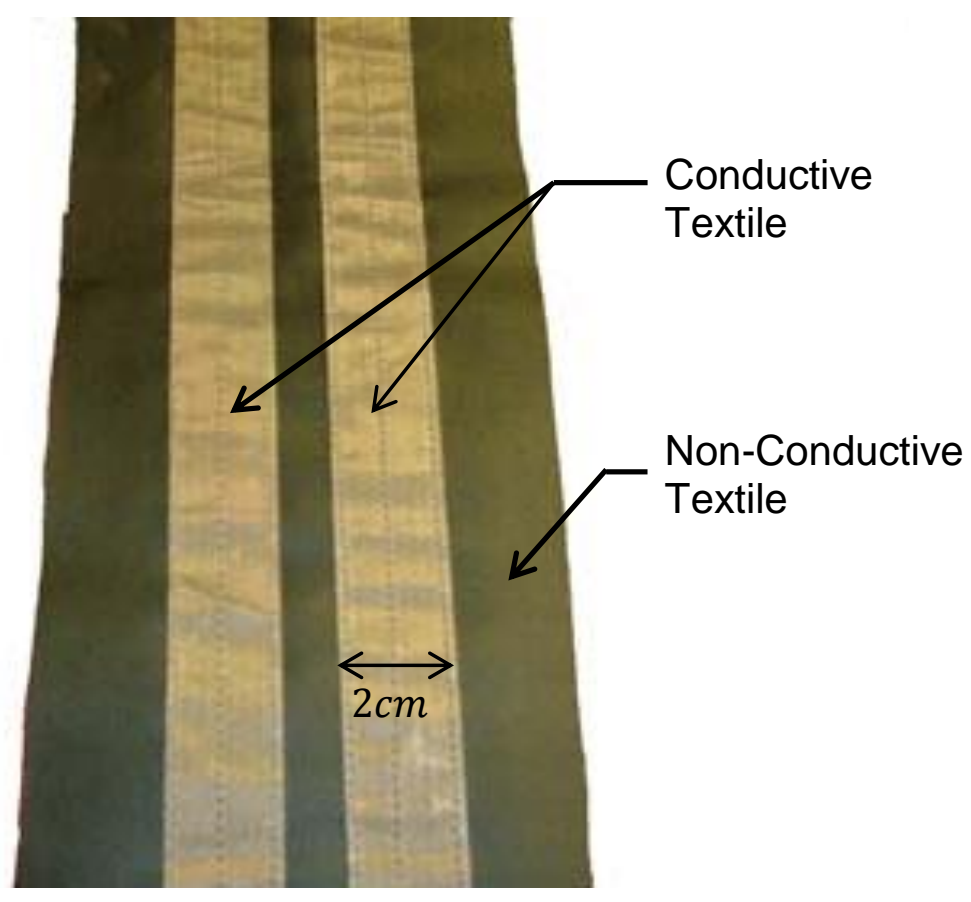

Figure 2.10 Textile transmission lines in which the conductive paths are made from a different electroconductive flat textile after Chedid et al [2.14]

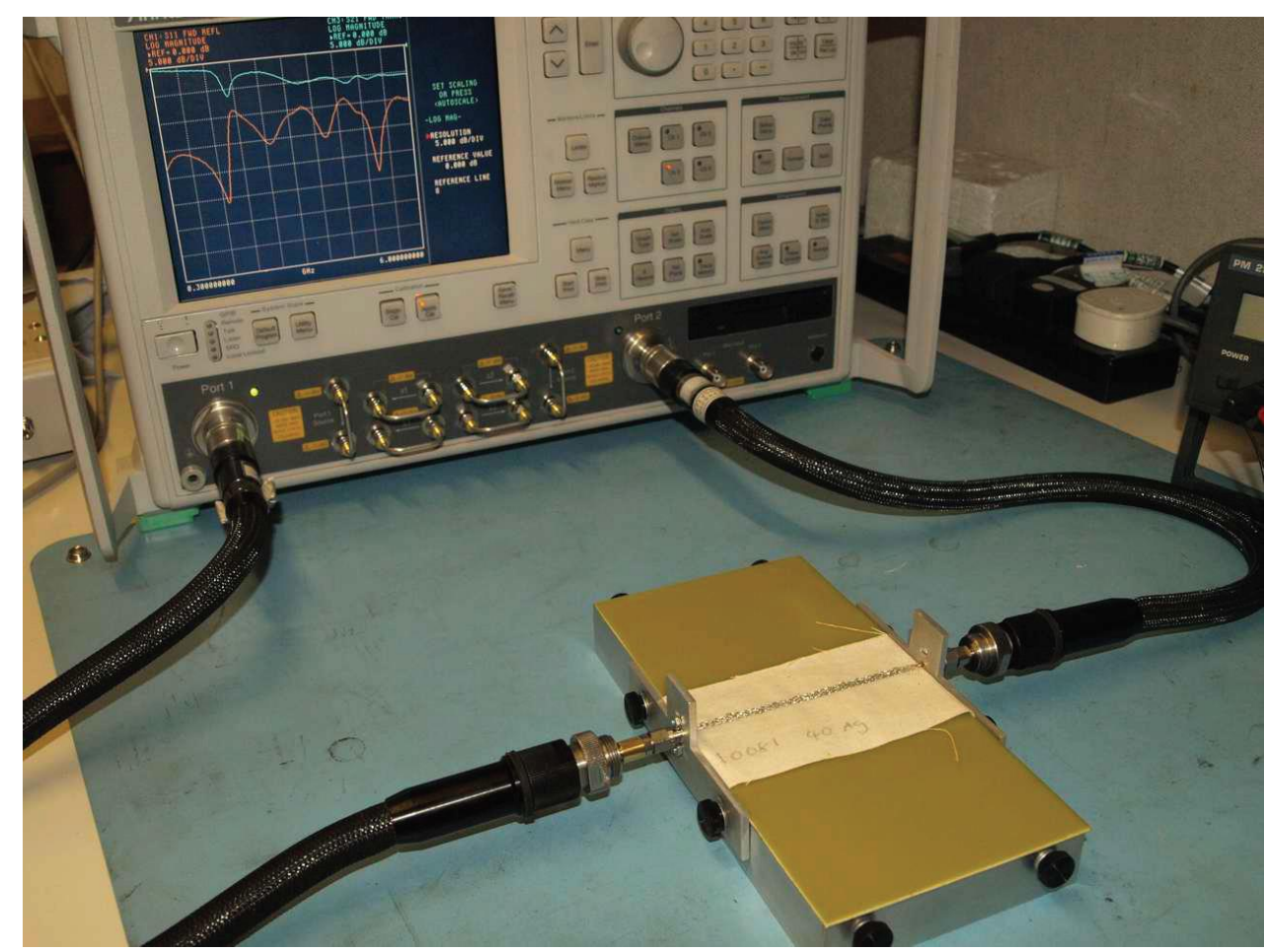

Figure 2.11 Conductive thread transmission line created through digital embroidery in jig above FR4 substrate after Acti et al. [2.15] 


\subsubsection{Conductive Threads for Signal Transmission}

Conductive threads are textile materials that can conduct electricity. Generally, they consist of a nonconductive fabric as substrate and a metal or carbon structure as a conductive component. They are preferred to metallic wires because of their light weight, flexibility and the capacity to use them in existing apparel using either sewing or embroidery machines. However, conductive threads have a poor conductivity compared to metallic conductors, silver $\left(\sigma=6.30 \times 10^{7} \mathrm{Sm}^{-1}\right.$ at $\left.20^{\circ} \mathrm{C}\right)$ [2.7] which is a better conductor compared to $\operatorname{copper}\left(\sigma=5.96 \times 10^{7} \mathrm{Sm}^{-1}\right.$ at $\left.20^{\circ} \mathrm{C}\right)$ [2.8]. One way to improve its conductivity is to make the amount of metal in the fabric composite bigger. But doing so makes it lose its typical textile properties like drapability and hand property, which refers to the way a fabric feels, which may also be rough, smooth, hard, cool, warm, soapy etc. Their poor conductivity alongside their molecular instability also makes conductive threads unsuitable for wiring [2.12].

\subsection{Fabrication of Conductive Threads}

Methods of fabricating conductive fibers include: filling of fibers with carbon or metal particles, coating of fibers with conductive polymers or metals and use of continuous or short fibers that are completely made of conductive materials [2.16]; while conductive threads are made from single or multiple strands of conductive and nonconductive fibers. The common methods of making these conductive threads are monofilament thread and multifilament thread which can be seen in Fig.2.12 (a-e) [2.22]. 


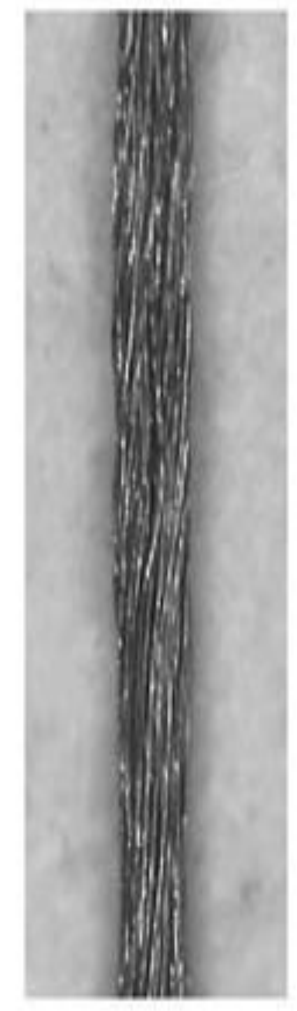

a

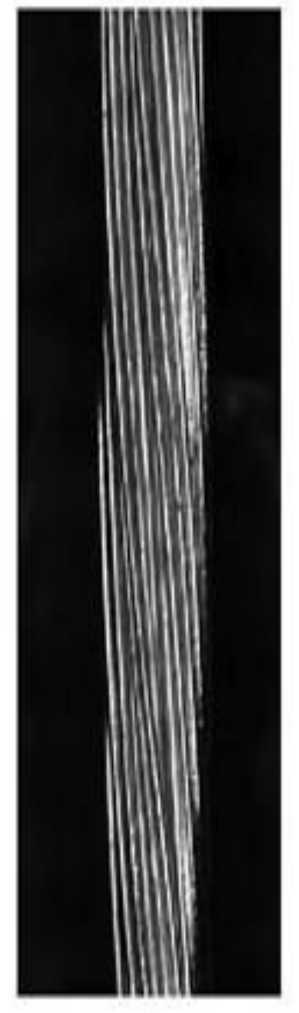

)
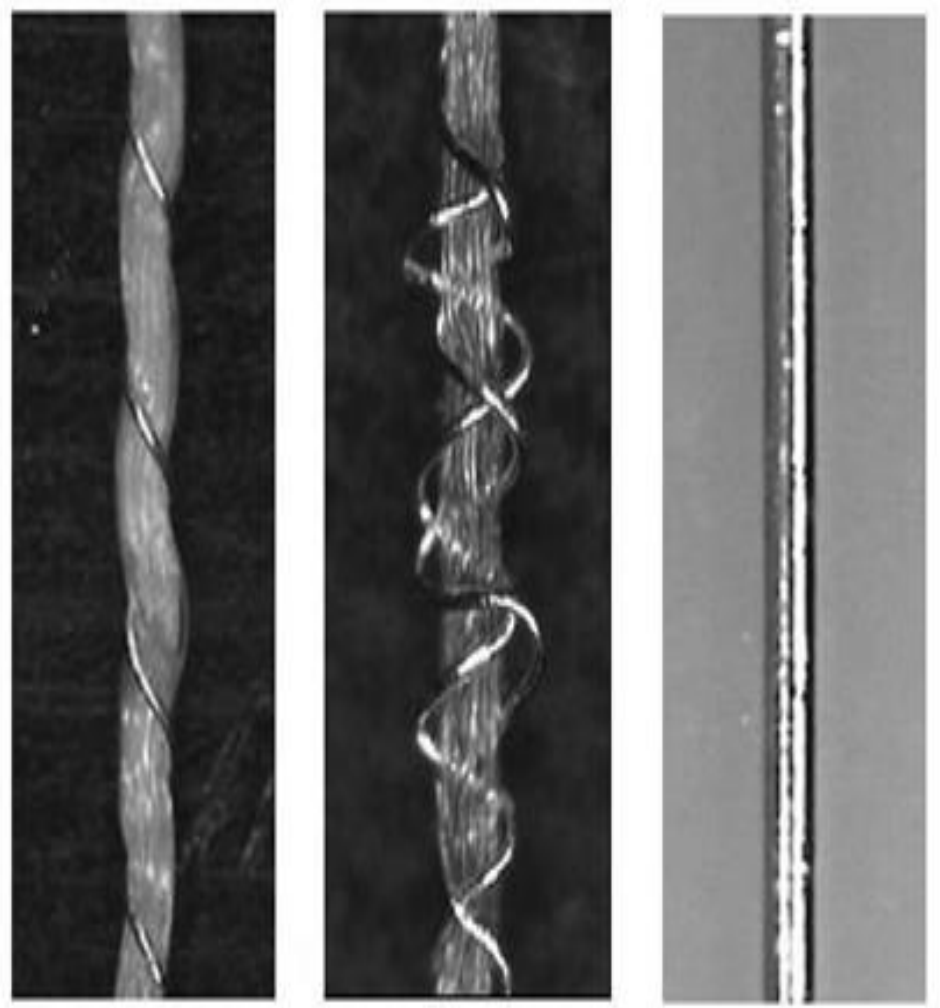

d

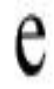

Figure 2.12 (a) Formed by twisting many thin elastic silver plated nylon fibres together (b) contains 60 copper fibres, each of which has a diameter of $40 \mu \mathrm{m}$, (c) and (d) are composite

threads of insulating and metallic fibres where both composite threads are created by spinning $40 \mu \mathrm{m}$ silver plated copper fibres around a nonconductive core which is composed of multiple nonconductive fibres and (e) is made up of single silver plated copper fibre with a diameter of $40 \mu \mathrm{m}$ after Ouyang and Chappell [2.22]

\subsection{Conductive Thread from Light Stitches}

In this thesis, a multifilament conductive thread from Light Stitches® (see Fig. 2.13) was used for stitching the transmission line. One single thread of Light Stitches comprises of approximately 96 individual filaments, each coated with a micron-thick layer of natural pure silver. 16 of these filaments are wound together to form an initial twist; two of these twists are then twisted together, and finally, three of these twists are combined. The thread has a resistance of $0.4 \Omega \mathrm{cm}^{-1}$ or $12 \Omega \mathrm{foot}^{-1}$. In thread terms, its thickness is approximately 18 
denier. The thread does not fray: there are no loose ends of filaments except where they have been cut and a spool contains approximately 162 metres [2.23]. A measurement of the DC resistance and cross sectional diameter of the conductive thread indicates a DC resistance of $0.43 \Omega \mathrm{cm}^{-1}\left(43 \Omega \mathrm{m}^{-1}\right.$ or $\left.13.1 \Omega \mathrm{ft}^{-1}\right)$ and a cross sectional diameter of $0.3 \mathrm{~mm}(0.3 \times$ $10^{-3} \mathrm{~m}$ ). Thus, the resistivity can be obtained, using,

$\rho=R \frac{A}{l}$

Where $R, A$ and $l$ are the resistance, area and length of the conductive thread respectively.

The calculated resistivity of the whole metallization from (2.47) is given as $1.2 \times 10^{-5} \Omega \mathrm{m}$,

while the conductivity which is given by $1 / \rho$ is $8.2 \times 10^{4} \mathrm{Sm}^{-1}$, which is quite low compared to silver with $\sigma=6.30 \times 10^{7} \mathrm{Sm}^{-1}$ [2.7] and copper with $\sigma=5.96 \times 10^{7} \mathrm{Sm}^{-1}$ [2.8] both at $20^{\circ} \mathrm{C}$ repectively. 


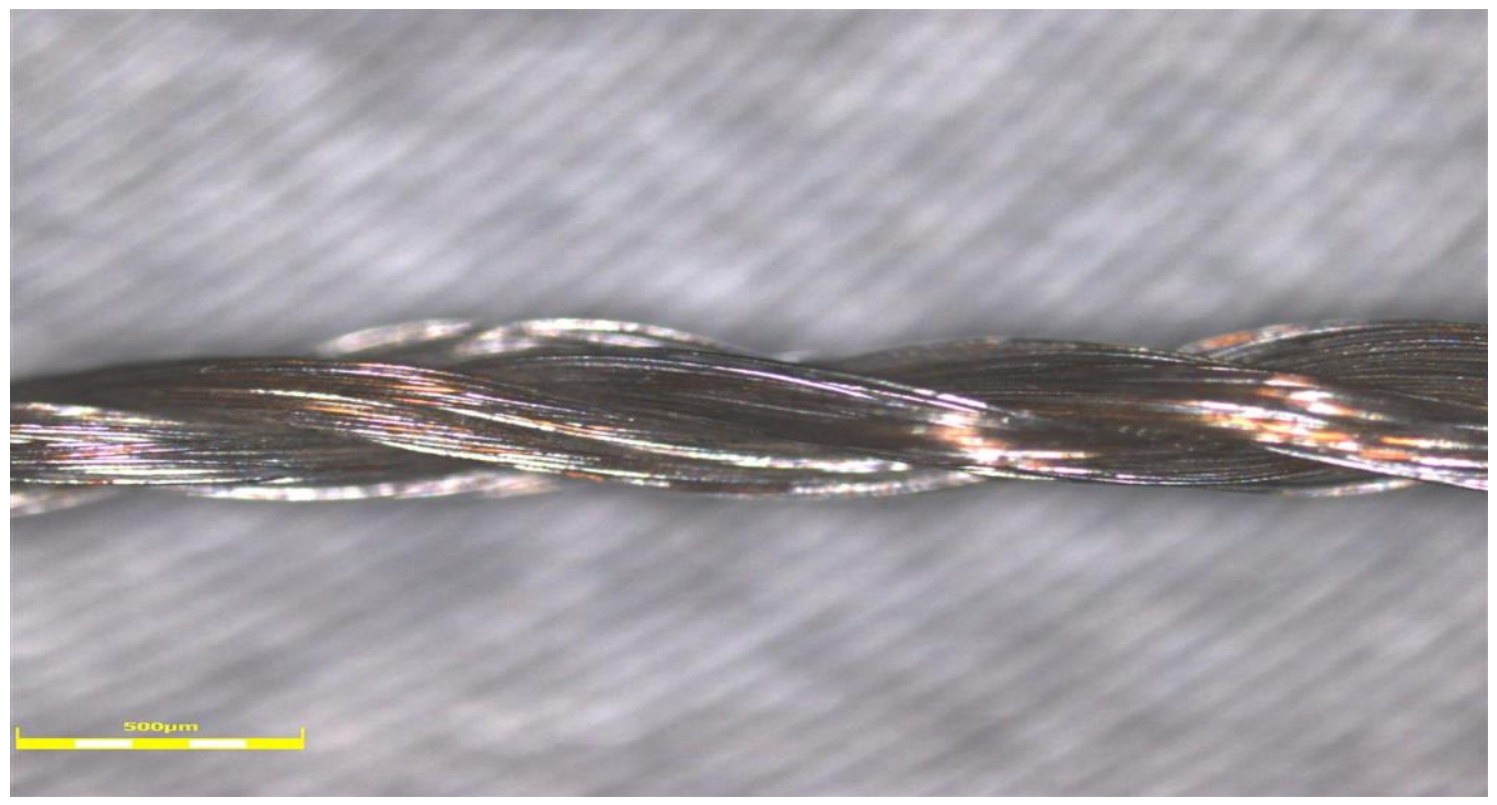

(a)

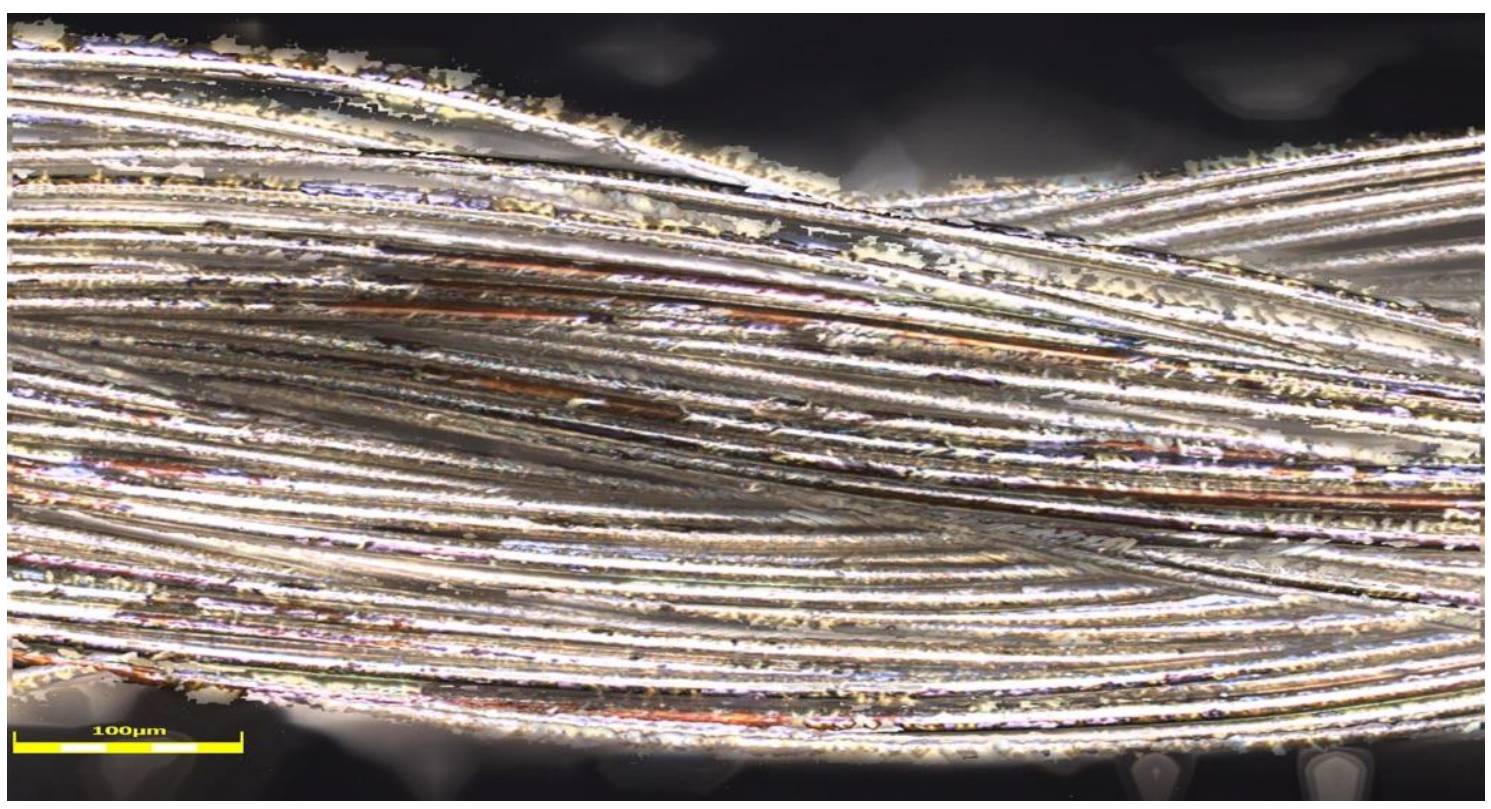

(b)

Figure 2.13 Colour image with High-dynamic-range imaging (HDR) of the Conductive thread from Light Stitches ${ }^{\circledR}$ using the Olympus LEXT Laser Confocal Microscope at (a) $500 \mu \mathrm{m}(\mathrm{b}) 100 \mu \mathrm{m}$ 


\subsection{Conclusion}

This chapter has presented an overview of transmission line theory. Discussions on transmission lines include transmission line parameters, equations, losses and signal attenuation. A review of wearable transmission lines is also presented, alongside discussions on wearable transmission lines, the use of conductive threads for signal transmission with a brief description of conductive threads from Light Stitches.

A basic knowledge of transmission theory is crucial to our understanding of the behavior of the stitched transmission line as it serves as a means of transporting RF signals between wearable communication equipment and to connect antennas to transmitters and receivers. Furthermore, because we are proposing a stitched transmission line which has poor shield coverage, with the shield made with mainly conductive threads, an understanding of the losses that are associated with transmission lines is significant to understanding its performance both at DC and RF level.

Chapter three presents an analysis on the shield of a coaxial cable with emphasis on Vance [2.4], Tyni [2.5] and Kley [2.6] models. 


\section{REFERENCE}

2.1. M. N. O. Sadiku, "Elements of Electromagnetics," Sixth Edition, Oxford University Press, 2014.

2.2. W. H. Hayt Jr. and J. A. Buck, "Engineering Electromagnetics," Sixth Edition, pp. 435, McGraw-Hill, 2001.

2.3. F. T. Ulaby, E. Michielssen and U. Ravaioli, "Fundamentals of Applied Electromagnetics," 6th Edition, pp. 48, Prentice-Hall, 1994.

2.4. G. P. Srivastava, "Microwave Circuits \& Components," pp.15 Defence Scientific Information \& Documentation Centre (DESIDOC), Defence R\&D Organisation, Metcalfe House, Delhi-110 054, 2004.

2.5. S. F. Adam and H. Packard, "Microwave Theory and Applications," pp. 7\&53, Eaglewood Cliffs: Prentice-Hall, 1969.

2.6. R. Ludwig and G. Bogdanov, "RF Circuit Design: Theory and Applications" 2nd edition, Prentice-Hall, 2000.

2.7. R. A. Serway, "Principles of Physics (2nd Ed.). Fort Worth, Texas; London: Saunders College Pub. pp. 602, 1998

2.8. D. Giancoli, "Electric Currents and Resistance," Physics for Scientists and Engineers with Modern Physics (4th Ed.). Upper Saddle River, New Jersey: Prentice Hall. pp. 658,2009

2.9. Losses in Transmission Lines [Online]. [Accessed: 12-February 2017]. Available from: http://www.tpub.com/neets/book10/41b.htm

2.10. Transmission Line Losses [Online]. [Accessed: 12-February 2017]. Available from: http://www.slideshare.net/krishnanrajesh/transmission-line-losses

2.11. J. Leśnikowski, "Textile Transmission Lines in the Modern Textronic Clothes," 89 Fibres \& Textiles in Eastern Europe, Vol. 19, No. 6 (89) pp. 89-93, 2011.

2.12. I. Locher, T. Kirstein, G. Troster. "Routing Methods Adapted to e-Textiles," ad. of Proc. 37th Int. Symp. Microelectron (IMAPS 2004), November 2004.

2.13. C. R. Merritt, B. Karaguzel, T. Kang, J. M. Wilson, P. D. Franzon, H.T. Nagle, B. Pourdeyhimi and E. Grant "Electrical Characterization of Transmission Lines on Specific Nonwoven Textile Substrates,” MRS Proceedings, 870, H4.7 doi:10.1557/PROC-870-H4.7, 2005. 
2.14. M. Chedid, P. Leisner and I. Belov, "Experimental analysis and modeling of the textile transmission line for wearable applications," International Journal of Clothing Science and Technology, Vol. 19 No. 1, pp. 59-71, 2007.

2.15. T. Acti, S. Zhang, A. Chauraya, W. Whittow, R. Seager, T. Dias and Y. Vardaxoglou, "High-Performance Flexible Fabric Electronics for Megahertz Frequency Communications', Loughborough Antennas \& Propagation Conference, pp. 1-4, November 2011.

2.16. D. Cottet, J. Grzyb, T. Kirstein and G. Tröster, "Electrical Characterisation of Textile Transmission Lines," IEEE Transaction on Advance Packaging, Vol. 26, N0. 2, pp. 182-190, May 2003.

2.17. T. Kirstein, D. Cottet, J. Grzyb and G. Tröster, "Textiles for Signal Transmission in Wearables," Proceedings Workshop on Modelling, Analysis and Middleware Support for Electronic Textiles MAMSET, San Jose, CA, 6, pp. 9-14, October 2002.

2.18. I. Locher and G. Tröster, "Screen-Printed Textile Transmission Lines," Textile Research Journal, Vol. 77(11), pp. 837-842, December 2007.

2.19. J. Jeon, S. Kim, J. Koo, S. Hong, Y. Moon, S. Jung and B. Kim, "Electrical Characterisation of Differential Stretchable Transmission Line," Microwave Symposium Digest (MTT), 2011 IEEE MTT-S International, vol., no., pp.1,1, 5-10 June 2011.

2.20. M. Choi and J. Kim, "Electrical Characteristics and Signal Transmission Characteristics of Hybrid Structure Yarns for Smart Wearable Devices," Fibres and Polymers, Vol.17, No.12, pp. 2055-2061, 2016

2.21. Z. Xu, T. Kaufmann, C. Fumeaux, "Wearable Textile Shielded Stripline for Broadband Operation," Microwave and Wireless Components Letters, IEEE, vol.24, no.8, pp.566-568, August 2014.

2.22. Y. Ouyang and W. J. Chappell, "High-Frequency Properties of Electro-Textiles for Wearable Antenna Applications," IEEE Transactions on Antennas and Propagation, vol. 56, no. 2, February 2008.

2.23. Conductive Thread Reel [Online]. [Accessed 12-February 2017]. Available from: http://www.lightstitches.co.uk/accessories/conductive-thread-reel-182m-approx.html

2.24. E. Vance, "Shielding effectiveness of braided-wire shields," IEEE Transactions on Electromagnetic Compatibility, vol. EMC-17, pp. 71-77, May 1975

2.25. M. Tyni, "The transfer impedance of coaxial cables with braided outer conductor," in Wroclaw EMC Symposium, 1976, pp. 410 - 419 
2.26. Thomas Kley, "Optimized single braided cable shields," IEEE Transactions on Electromagnetic Compatibility, vol. 35, no. 1, February 1993 


\section{Chapter 3}

\section{Analysis on the Shield of a Coaxial Cable}

In this chapter discussion on the shield of a braided coaxial cable is been presented. The coaxial cable has two distinct properties, which includes guidance of TEM waves within the structure and screening against coupling which is affected by the shield coverage. The shield is very significant in guarding against electrical noise affecting the signals being transmitted through the cable and also aid in reducing electromagnetic radiation from leaving the cable and interfering with other devices thereby acting as electrical noise. Furthermore, the shield helps in minimizing capacitively coupled noise from other electrical sources. This project aims at using the idea of a braided coaxial cable to develop a stitched transmission line. The proposed stitched transmission is comparable to the braided coaxial cable in terms of its inner conductor, tubular dielectric, and stitched shield. However, the coverage of the stitched shield of the stitched transmission line is quite low when compared to a standard braided coaxial cable. An attractive proposition for the stitched wearable transmission line would be to have the shielding and propagation characteristics of a standard coaxial line but there are significant obstacles to introducing this. In this chapter, the shielding effectiveness of a coaxial line will be considered with a view to understanding how this can be applied in a wearable situation. A study on the shielding effectiveness and transfer impedance of a braided coaxial cable with emphasis on Vance [3.1], Tyni [3.2] and Kley models [3.3] are presented.

\subsection{Coaxial Cable}

Coaxial cable is a type of transmission line made up of two conductors separated by a dielectric material. Typical dielectric materials include polyethylene (PE), polypropylene (PP), fluorinated ethylene propylene (FEP), and polytetrafluoroethylene (PTFE). The inner conductor and the outer conductor (referred to as the shield), are configured in such a way that they form concentric cylinders with a common axis, hence the name coaxial cable meaning having a common axis. The shield is usually earthed and provides the return path for signals transmitted through it. Depending on the construction of the shield, a coaxial cable 
can be classified into two categories namely; the rigid or semi-rigid coaxial cable made up of a solid screen (a pipe) and the flexible coaxial cable made up of braided wires. The focus here is on the coaxial cable with the braided shield as that applies closely to the proposed stitched transmission line. It would be attractive to have a more compact coaxial-like line that can be stitched into a garment. However, the limitation on its flexibility due to the tightly braided screen is a deterrent. The concept being explored in the next chapter is to use a stitched screen with a conventional inner conductor and tubular dielectric medium.
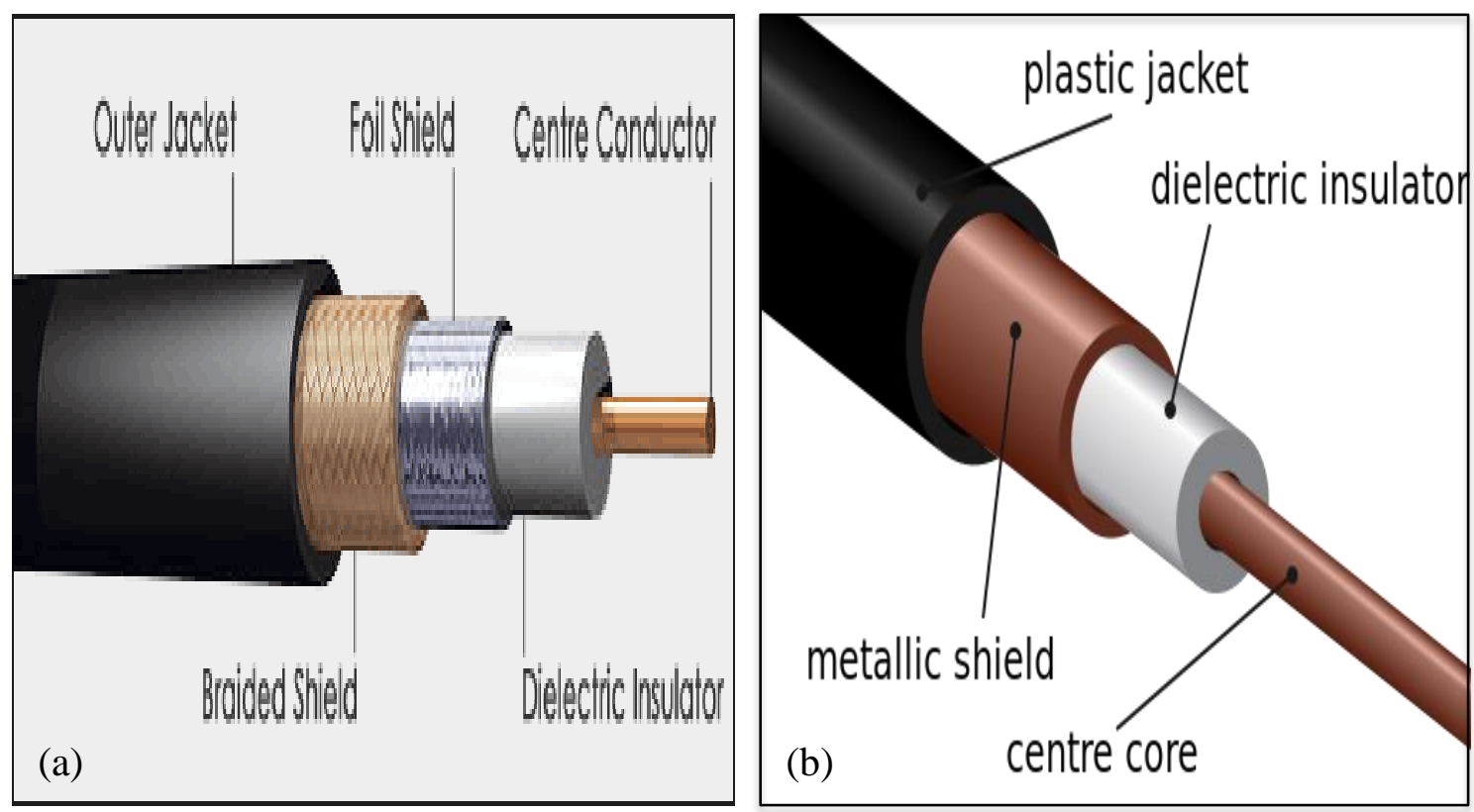

Figure 3.1(a) Flexible coaxial cable [3.4] (b) Rigid coaxial cable [3.5]

\subsubsection{Role of the shield}

The shield which forms the outer conductor of a coaxial cable acts to first keep the desired currents inside the coaxial cable, secondly to keep the undesired currents outside of the coaxial cable, thirdly to act as a current return path for the signal, however it is also important to note here that at high frequencies a good proportion of the power travels in the fields, and lastly to provide a path to earth ground for external or interfering field on the coaxial cable. 


\subsection{Theoretical Evaluation of Cable Shielding}

Evaluation of signals transmitted through the shield of a coaxial cable can be carried out using the following parameters:

- Shielding effectiveness of cable shields

- Transfer impedance of cable shields

\subsubsection{Shielding Effectiveness of a Braided Cable Shield}

The shielding effectiveness, $S_{\text {eff }}$, of a cable is a measure of the quality of its shielding. This depends largely upon the coverage afforded by the shield and the thickness of the shield material. More shield coverage means less radiation of energy. However this does not necessarily mean less signal attenuation. Leakage of energy into cables when subjected to external interference from electromagnetic fields has been discussed in [3.6].

Most cables are braided shield largely because of its flexibility, strength, durability and long flex life span. Typical braids provide $60 \%-98 \%$ coverage and are less effective compared to solid shields. Braided shields usually provide just slightly reduced electric field shielding (except at UHF frequencies) but greatly reduced magnetic field shielding. The reason is that braid distorts the uniformity of the longitudinal shield current. A braid is typically from 5 $30 \mathrm{~dB}$ less effective than a solid shield for guarding against magnetic fields. At higher frequencies, the effectiveness of the braid decreases even more as a result of the gaps or holes that exist between the weave. Multiple shields offer more protection but at a higher cost and less flexibility [3.7]-[3.8].

Shielding can be expressed in terms of the reduction in magnetic and or electric field strength caused by the shield. Thus the effectiveness of a shield can be viewed as the ratio of the magnitude of the magnetic field or electric field that is incident on the shield to the magnetic or electric field that is transmitted as it emerges from the shield, expressed in decibels (dB).

$$
\begin{aligned}
& S_{\text {eff }}=20 \log \frac{H_{i}}{H_{t}} d B \\
& S_{\text {eff }}=20 \log \frac{E_{i}}{E_{t}} d B
\end{aligned}
$$

Conversely, this chapter focusses primarily on the use of surface transfer impedance to determine the shielding effectiveness of a braided coaxial cable and also contributions made by Vance [3.1], Tyni [3.2] and Kley [3.3] towards the development of surface transfer impedance of a braided coaxial cable. 


\subsubsection{Transfer Impedance of a Braided Coaxial Cable Shield}

Transfer impedance is a measure of shielding effectiveness of a braided coaxial cable used to determine the shielding effectiveness against both ingress and egress of interfering signals. It relates a current on the surface of the shield to the voltage drop generated by its current on the opposite surface of the shield. Generally, shield with lower DC resistance will have a lower voltage drop and lower transfer impedance. In the following sections, a discussion on the three models as proposed by Vance [3.1], Tyni [3.2] and Kley [3.3] are been discussed.

\subsubsection{Vance Model}

Following the analysis on the theory of coupling through electrically small apertures of braided shields presented by Kaden and Markowitz in [3.9]-[3.10], Vance in [3.1] presented a description of the braided shield in terms of the following parameters:

- Mean diameter of the metal braid shield, $D_{m}$

- Diameter of a single wire, $d_{w}$

- Number of carriers crossing per unit length, $C_{c}$ (belt of wires $C_{c}=1,2, \ldots$ )

- $\quad$ Number of wires, $N_{w}$ (with $\left.N_{w}=1,2, \ldots\right)$

- Weave angle, $\alpha_{w}$

- The conductivity of the wires that make up the shield, $\sigma$

From which the coverage factor can be defined as:

$F=\frac{N_{w} C_{c} d_{w}}{\sin \alpha_{w}}$

The shield coverage is given,

$x=2 F-F^{2}$

While the number of holes per unit length of the metallic braid

$v=\frac{2 \pi D_{m} \sin \alpha \cos \alpha}{N_{w}^{2} d_{w}^{2}} F^{2}$

The weave angle, $\alpha_{w}$, plays a significant role in defining the shape of the braid and its magnetic coupling, which largely affects the value of the transfer impedance, $Z_{T}$. Suppose the weave angle $\alpha<45^{\circ}$, the major axis of the diamond shaped apertures is perpendicular to the magnetic field and the shape is oriented for minimum magnetic coupling through the aperture of the shield. 


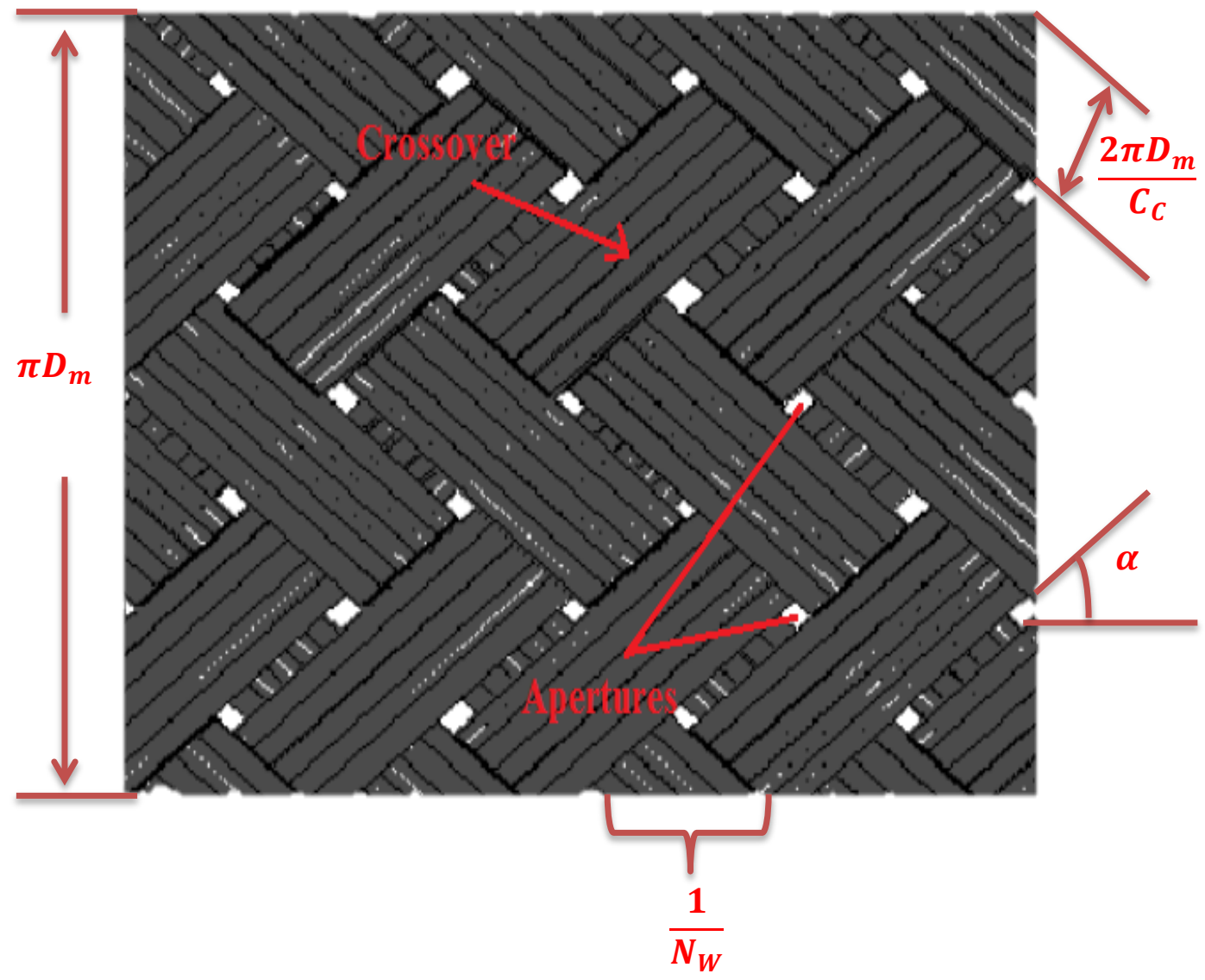

Figure 3.2 Weave pattern of a braided shield coaxial cable after Vance [3.1]

The coupling on a braided shield was approximated to that on a perforated solid tubular shield, assuming that the current flows longitudinally on the braid. The transfer impedance of the braided shield consists of two components; the first determines the diffusion of the electromagnetic energy through the shield, while the second component determines the penetration of the magnetic field through the shield aperture.

Furthermore, the diffusion term of the low-frequency resistive component $R_{D C}$ of the transfer impedance $Z_{T}$ was estimated by assuming that each conductor of diameter, $d$, is isolated from all other conductors, where the contact resistance between the cables is large compared to the wire resistance. 
$R_{C}=\frac{4}{\pi d_{w}^{2} \sigma \cos \alpha}$

The DC resistance per unit length of the shield is given by:

$R_{D C} \approx \frac{R_{C}}{N_{W} C_{C}}=\frac{4}{N_{W} C_{C} \pi d_{w}^{2} \sigma \cos \alpha}$

The woven construction of the braid influences the DC resistance of the shield, thus a higher weave angle leads to more wire turns.

Assuming the DC resistive component behaves in the same as the diffusion term in the cylindrical tube with thickness, $d$, and the skin depth effect implemented along with the DC resistance of the braid, with the total screen impedance given by:

$Z_{D}=R_{D C} \frac{\frac{(1+j) d_{w}}{\delta}}{\sinh \left[\frac{(1+j) d_{w}}{\delta}\right]}$

Where, $\delta$, the skin depth is given by:

$\delta=\sqrt{\frac{2}{\omega \mu_{0} \sigma}}$

By analogy with perforated solid shield, the mutual inductance term for $v$, holes per meter, is given by:

$M_{12}=v \frac{\mu_{0} m}{4 \pi^{2} b^{2}}$

From data for similar shapes such as ellipses and apertures with semi-circular ends, the polarizability of the diamond-shaped aperture can be represented by the polarizability of an equivalent elliptical hole [3.10]. The magnetic polarizability of an aperture has been derived in closed form for the magnetic fields parallel to either axis of the ellipse. Thus the magnetic polarizability for the magnetic fields parallel to the major axis is as Eq. (3.11), while the magnetic fields parallel to the minor axis is given by Eq. (3.12) for an ellipse of eccentricity as in Eq. (3.13):

$$
\begin{aligned}
& m_{\ell}=\frac{\pi \ell^{3}}{24}\left(\frac{e^{2}}{K(e)-E(e)}\right) \\
& m_{w}=\frac{\pi \ell^{3}}{24}\left(\frac{\left(1-e^{2}\right) e^{2}}{E(e)-\left(1-e^{2}\right) K(e)}\right)
\end{aligned}
$$


$e=\left[1-\left(\frac{w}{\ell}\right)^{2}\right]^{\frac{1}{2}}$

Where $\ell$ is the major axis and $w$ is the minor axis; $K(e)$ and $E(e)$ are complete integrals of the first and second kind respectively defined by:

$K(e)=\int_{0}^{\frac{\pi}{2}} \frac{d \varphi}{\sqrt{\left(1-\sin ^{2} \varphi\right)}}$

$E(e)=\int_{0}^{\frac{\pi}{2}}\left[\sqrt{\left(1-e^{2} \sin ^{2} \varphi\right)}\right] d \varphi$

Suppose the braided shield pattern can be simulated by ellipses with similar major and minor axes to the rhombic apertures, the mutual inductive coupling which represents $Z_{T}$ associated with the apertures could be written as:

$M_{12} \approx \frac{\pi \mu_{0}}{6 C_{C}}(1-k)^{\frac{3}{2}}\left\{\frac{e^{2}}{K(e)-\left(1-e^{2}\right) E(e)}\right\} ; \alpha<45^{\circ}$

$$
e=\sqrt{\left(1-\tan ^{2} \alpha\right)}
$$

$M_{12} \approx \frac{\pi \mu_{0}}{6 C_{C}}(1-\mathcal{X})^{\frac{3}{2}}\left\{\frac{\frac{e^{2}}{\sqrt{\left(1-e^{2}\right)}}}{K(e)-E(e)}\right\} ; \alpha>45^{\circ}$

$e=\sqrt{\left(1-\cot ^{2} \alpha\right)}$

Finally, the total transfer impedance of the braided shield is given as:

$Z_{T}=Z_{D}+j \omega M_{12}$

\subsubsection{Tyni Model}

Tyni in [3.2] proposed that the current direction in the braided coaxial cable is not parallel to the cable axis but rather follows the wires in a helix-like manner. This porpoise [3.11] behavior led to an extra coupling between the inner and outer braid layers and hence the inclusion of a third term the braid inductance, $L_{b}$, in the transfer impedance expression as presented by Vance in [3.1] which only considered the hole inductance, $L_{h}$. The idea behind the braid inductance, $L_{b}$, is that it is treated as an inductance between two interlaced halves of the braid, relating it to the geometry of the braid. Thus $L_{b}$ and $L_{h}$ can be written as in [3.2]: 
$L_{b}=\frac{\mu_{0} h_{r}}{4 \pi D_{m}}\left(1-\tan ^{2} \alpha\right)$

$L_{h}=\frac{2 \mu_{0} N_{w}}{\pi \cos \alpha}\left(\frac{b}{\pi D_{m}}\right)^{2} e^{\left(\frac{-\pi d}{b}-2\right)}$

With reference to Fig.3.2, the following parameters are given as:

$l=\frac{N_{w}}{2 C_{C}}$

$\alpha=\tan ^{-1}\left(\frac{2 \pi D_{m} C_{C}}{N_{w}}\right)$

$D_{m}=D_{0}+2 d_{w}$

$g=\frac{2 \pi D_{m}}{C_{C}} \cos \alpha-n d_{w}$

$h=\frac{2 d_{w}^{2}}{g+d_{w}}$

A further modification was submitted in [3.3] with Eq. (3.27) and (3.28) suggesting that $D_{m}$ is also a function of $h_{r}$. The equations denote a non-linear relationship with a solution found by using Newton-Raphson iteration process (see Appendix C).

$D_{m}=D_{0}+2 d_{w}+h_{r}$

Where $h_{r}$ can be obtained through:

$\left[\frac{2 \pi}{C_{C}}\right]+\left[(1-n) d_{w}+\frac{2 \pi}{C_{C}}\left(D_{0}+2 d_{w}\right) \cos \alpha\right] h_{r}-2 d_{w}^{2}=0$

Hence $Z_{T}$ can be expressed as:

$Z_{T}=Z_{D}+j \omega\left(L_{b}+L_{h}\right)$

\subsubsection{Kley Model}

Kley in [3.3] observed that the techniques for calculating the internal coupling parameters of a single braided shield as proposed in [3.1] and [3.2] were inconsistent with measured results.

Considering Tyni's model [3.2], which is given by Eq. (3.29), Kley considered Shelkunoff's formula [3.9] for the transfer impedance of an equivalent tube with thickness $d_{R}$ and 
supposed from literature [3.1] that $d_{R}=d$ and that two factors, the mutual inductance due to the apertures, $M_{H}$ and the mutual inductance between the carriers in the braid, $M_{B}$, contribute to the coupling inductance, $M_{T}$.

$M_{T}=M_{H}+M_{B}$

Kley in [3.3] attuned an approach described by Vance in [3.1] for the hole inductance, emanating from the mutual coupling due to the apertures in the braid derived from the magnetic polarizability of the elliptical aperture, and approximated this elliptic function in Eq. (3.17) by $2-\cos \alpha$, with an exponential attenuation factor of $\tau_{H}$, and also considering the factor of 0.875 due to the curvature of the braid to give:

$M_{H} \approx \mu_{0} 0.875 \frac{\pi(2-\cos \alpha)}{N_{w}}(1-F) e^{-\tau_{H}}$

Where

$\tau_{H}=9.6 F \sqrt[3]{\frac{x^{2} d_{w}}{D_{m}}}$

Assuming proximity effects can be ignored and the current distribution in the shield is symmetrical, the hole inductance for circular apertures can be related to the braid parameters alone.

The braid inductance varies with the braid angle $\alpha$ with no induced voltage if $\alpha=45^{\circ}$ and a maximum induced voltage if $\alpha=0^{\circ}$. Also from Eq. (3.17), the braid inductance in Eq. (3.20) was validated by triaxial configuration measurement in [3.12] to give an approximate formula as derived by Kley:

$M_{B} \approx-\mu_{0} \frac{0.11}{N_{w} n} \cos \left(2 K_{1} \alpha\right)$

Where

$K_{1}=\frac{\pi}{4}\left[\frac{2}{3} F_{0}+\frac{\pi}{10}\right]^{-1}$

Here $F_{0}$ represents the minimal fill factor of the braid derived from Eq. (3.17), given by:

$F_{0}=F \cos \alpha$ 
A third inductance called the skin inductance, $M_{S}$ is introduced which is due to eddy current on the surface of the rhombic apertures as the magnetic field penetrates through the apertures of the braid.

$M_{S}=\frac{1}{\omega \pi \sigma \delta}\left[D_{L}^{-1}+D_{G}^{-1}\right]$

Where $D_{L}$ and $D_{G}$ are fictitious diameters that model the corresponding skin effect. The evaluation of the measurements carried out by Kley [3.3] yields an approximate formula for $D_{L}$ given by:

$D_{L}^{-1} \approx \frac{10 \pi F_{0}^{2} \cos \alpha}{D_{m}}[1-F] e^{-\tau_{E}}$

With the attenuation factor given by:

$\tau_{E}=12 F \sqrt[3]{\frac{x^{2} d_{w}}{D_{m}}}$

And the fictitious parameter $D_{G}$ approximated by:

$D_{G}^{-1} \approx-\frac{3.3}{2 \pi F_{0} D_{m}} \cos \left(2 K_{2} \alpha\right)$

Where

$K_{2}=\frac{\pi}{4}\left[\frac{2}{3} F_{0}+\frac{3}{8}\right]^{-1}$

Evaluation of measurements by Kley [3.3] indicates that the thickness of the equivalent tube is proportional to the wire diameter, $d_{w}$, and the thickness found to be slightly dependent on the braid angle, $\alpha$. From Eq. (3.8), the screen impedance of the tube can be equated as:

$Z_{R}=R_{D C}\left[\frac{\frac{d_{R}(1+j)}{\delta}}{\sinh \left(\frac{d_{R}(1+j)}{\delta}\right)}\right]$

Where

$d_{R}=\frac{0.67 d_{w}}{\sqrt{\cos \alpha}}$ 
Finally, the approximate formula for the transfer impedance of a single-braided shield can be written as:

$Z_{T}=Z_{R}+j \omega M$

Where

$M=M_{H}+M_{B}+M_{S}$

\subsection{Application of Kley Model to the Stitched Transmission Line}

A comparison of the three models indicates that Kley's model [3.3] is most precise in modeling transfer impedance of braided shield cables as it contains all the possible features of inductances within the braid, with research, carried out by Thomas et al. confirming that in [3.14]. The Kley model [3.3] is made up of two sections, the first representing the diffusion of electromagnetic energy through the metal braids $Z_{D}$ and the second, $j \omega M$, representing the leakage of magnetic fields through the metal braids. The diffusion component, $Z_{D}$, of the metal braid is governed by the DC resistance of the metal braid and diffusion waves through the wall of the cylindrical braid. The inductance, $M$, from Kley [3.3] in Eq. (3.44) is a superposition of the hole inductance, $M_{H}$, braid inductance, $M_{B}$, and the skin inductance, $M_{S}$, unlike the Vance model [3.1] whose inductance, $M$, is made up of the hole inductance, $M_{H}$, and Tyni model [3.2] whose inductance, $M$, is made up of the hole and braid inductance, $M_{H}$ and $M_{B}$. Hence the Kley model can be seen as a significant improvement to both the Vance [3.1] and Tyni models [3.2].

At low frequencies $(<1 G \mathrm{~Hz})$, the transfer impedance, $Z_{T}$, is dominated by a wave diffusion process which is inversely proportional to the frequency (i.e. $Z_{D}$ is dominant). While at high frequency, the fields' coupling through the apertures and the porpoising of the braid takes over (i.e. the hole inductance, $M_{H}$, braid inductance, $M_{B}$ and skin inductance, $M_{S}$ are dominant).

With the stitched transmission line proposed in chapter four modeled as a braided coaxial cable with two helices, which is synonymous to a composite counter-wound helical structure with a concentric conductor proposed by Wait in [3.15], the Kley model [3.3] is best applicable to the stitched transmission line. The current flow on the shield of the proposed stitched transmission line is proposed to follow the conductive threads in a helix-like manner, 
inside and outside the shield called porpoising as presented by Tyni [3.2] and Kley [3.3], contrary to Vance [3.1], where the current flow on the shield of the braided coaxial is approximated to flow parallel to the axis. The transfer impedance of the stitch transmission line is thus made up of two sections. With the first representing the diffusion through the stitch shield and the second representing the leakage of magnetic fields through the stitch shield.

\subsection{Conclusion}

In this chapter, analytical models for the calculation of the transfer impedance for braided shield cable have been discussed. A comparison of the three models is made and the Kley model is agreed to be the best applicable to the stitched transmission line.

The Kley model would be applied to the stitched transmission line in chapter five, to determine the transfer impedance of the stitched transmission line for low frequencies $(<$ $1 G H z$ ), where, the transfer impedance, $Z_{T}$, is dominated by the wave diffusion process, $Z_{D}$.

Chapter four will present the design and construction of the stitched transmission line using an improved presser foot. 


\section{REFERENCE}

3.1. E. Vance, "Shielding effectiveness of braided-wire shields," IEEE Transactions on Electromagnetic Compatibility, vol. EMC-17, pp. 71-77, May 1975

3.2. M. Tyni, "The transfer impedance of coaxial cables with braided outer conductor," in Wroclaw EMC Symposium, 1976, pp. 410 - 419

3.3. Thomas Kley, "Optimized single braided cable shields," IEEE Transactions on Electromagnetic Compatibility, vol. 35, no. 1, February 1993

3.4. Electrical Cable, [Online]. [Accessed: 12-February 2017]. Available from: http://www.noblemc.co.za/Suppliers/CoaxialCableSuppliers.aspx

3.5. Coaxial cable, [Online]. [Accessed: 12-February 2017]. Available from: https://en.wikipedia.org/wiki/Coaxial_cable

3.6. S. Sali, F.A. Benson and J. E. Sitch, "General crosstalk equations between two braided coaxial cables in free space," IEE Proceedings, vol. 130, pt. a, no. 6, September 1983

3.7. H. W. Ott, "Electromagnetic Compatibility Engineering," John Wiley \& Sons, Inc., Publication, pp.79, 2009

3.8. B. C. Gabrielson, “TEMPEST Engineering and Hardware Design.” [Online]. Security Engineering Services, Chesapeake Beach, MD 20732, pp.14.4, 1998, [Accessed: 12February 2017]. Available from: http://surflibrary.org/ses/TEMPENG/CH14ShieldedWires.pdf

3.9. S. A. Schelkunoff, "The electromagnetic theory of coaxial transmission lines and cylindrical shields,” Bell Syst. Tech. J., 1934, 13, (14), pp. 532 - 579

3.10. S. B. Cohn, "Determination of Aperture Parameters by Electrolytic Tank Measurements,” Proc. IRE., Vol. 39, pp. 1416-1421, November 1951

3.11. J. N. Katakis, "Transfer Impedance of Wire Braided Coaxial Cables at Radio and Microwave Frequencies," MEng thesis, University of Sheffield, Feb. 1983

3.12. Thomas Kley, "Measuring the coupling parameters of shielded cables", IEEE Trans. in EMC, vol. 35, no. 1, pp. 10 - 20, Feb. 1993

3.13. T. Y Xian, "Modelling of interconnects including coaxial cables and multiconductor lines", Ph.D. thesis, University of Nottingham

3.14. D. W. P. Thomas, C. Christopoulos, F. Leferink and H. Bergsma, "Practical measure of cable coupling," 2009 International Conference on Electromagnetics in Advanced Applications, Torino, pp. 803-806, 2009 
3.15. J. R. Wait, "Electromagnetic Theory of the Loosely-Braided Coaxial Cable: Part I," in IEEE Transactions on Microwave Theory and Techniques, vol. 24, no. 9, pp. 547553, September 1976 


\section{Chapter 4}

\section{Numerical modeling and fabrication of the Stitched Transmission Line}

This chapter presents the design and construction of a stitched transmission line using the idea of a braided coaxial cable. The proposed stitched transmission line which is built with the aid of a modified presser foot and a Singer Talent ${ }^{\circledR}$ sewing machine is very practical, fast and easy to install and economical. Here, the geometry, full field modeling with three stitch lengths corresponding to three stitch angles alongside the construction of the stitched transmission line are presented. A description of the improved presser foot used in the fabrication of the stitch transmission line is also introduced.

\subsection{Stitched Transmission Line for Wearable RF Devices}

Wearable transmission lines which are means of transporting RF signals between various pieces of communication equipments and to connect wearable antennas to transmitters and receivers have been studied in recent literature. Didier et al. [4.1] and Kirstein et al. [4.2] presented a study on the characterization of textile transmission lines for use in wearable computing applications. Embroidery techniques use in fabricating transmission lines has been considered in [4.3], while the use of twisted copper yarns made with yarn covering process, used to transmit signals and power has been studied in [4.4]. Screen printed textile lines [4.5] and textile shielded striplines [4.6] have been presented for broadband operations. Further reading on textile transmission lines can be seen in [4.7] - [4.9]. The washability and stretchability effects on textile transmission lines have also been studied in [4.10] and [4.11]. Unfortunately, authors have ignored the impacts of ambient parameters such as temperature variations on stretchability and state of the stitches on the electrical parameters of textile transmission lines. However, in [4.12], preliminary studies conducted by Leśnikowski suggested that humidity of the substrate of a textile transmission line has a significant impact on the ability of the transmission line to transmit signals within a wide spectrum of frequencies.

This research work aims to investigate the feasibility of using the idea of a braided coaxial cable in developing a stitched transmission line and also study the performance of 
transmission line when stitched with conductive threads and copper wires and with conductive threads only. Numerical modelling and VNA measurement as presented in chapter five were carried out on the stitched transmission line with three different stitch lengths (corresponding to three different stitch angles), with three different stitch types, two different substrates Denim and Felt; when washed and when bent through curved angles of $90^{\circ}$ and $180^{\circ}$, and when placed within the presence of the human body. An approximate geometry of the stitched transmission line alongside the constructed structure can be seen in Fig. 4.1(a) and (b). The sparsely covered stitched transmission line is made up of a stripped RG 174, a denim substrate, and a stitched shield. Despite having a higher signal loss compared to larger diameter cables such as RG58, the RG174 was chosen because of its flexibility and smaller diameter which makes it a good candidate for use with the novel presser foot. Usually the choice of a coaxial cable for specific applications involves a concession between RF losses, leakage, overall diameter, weight, flexibility and cost. Conductive threads from Light Stitches ${ }^{\circledR}$ were used as the shield. The substrate size is $150 \mathrm{~mm} \times 100 \mathrm{~mm}$, while the length of the stitched transmission line is $150 \mathrm{~mm}$. The proposed stitched transmission line is very practical, fast and easy to install, economical and has sufficient frequency range to support multiple channel. A close look at the textile geometry of the shield of the stitched transmission line indicates the stitches follow a helical path around the stitched line. 


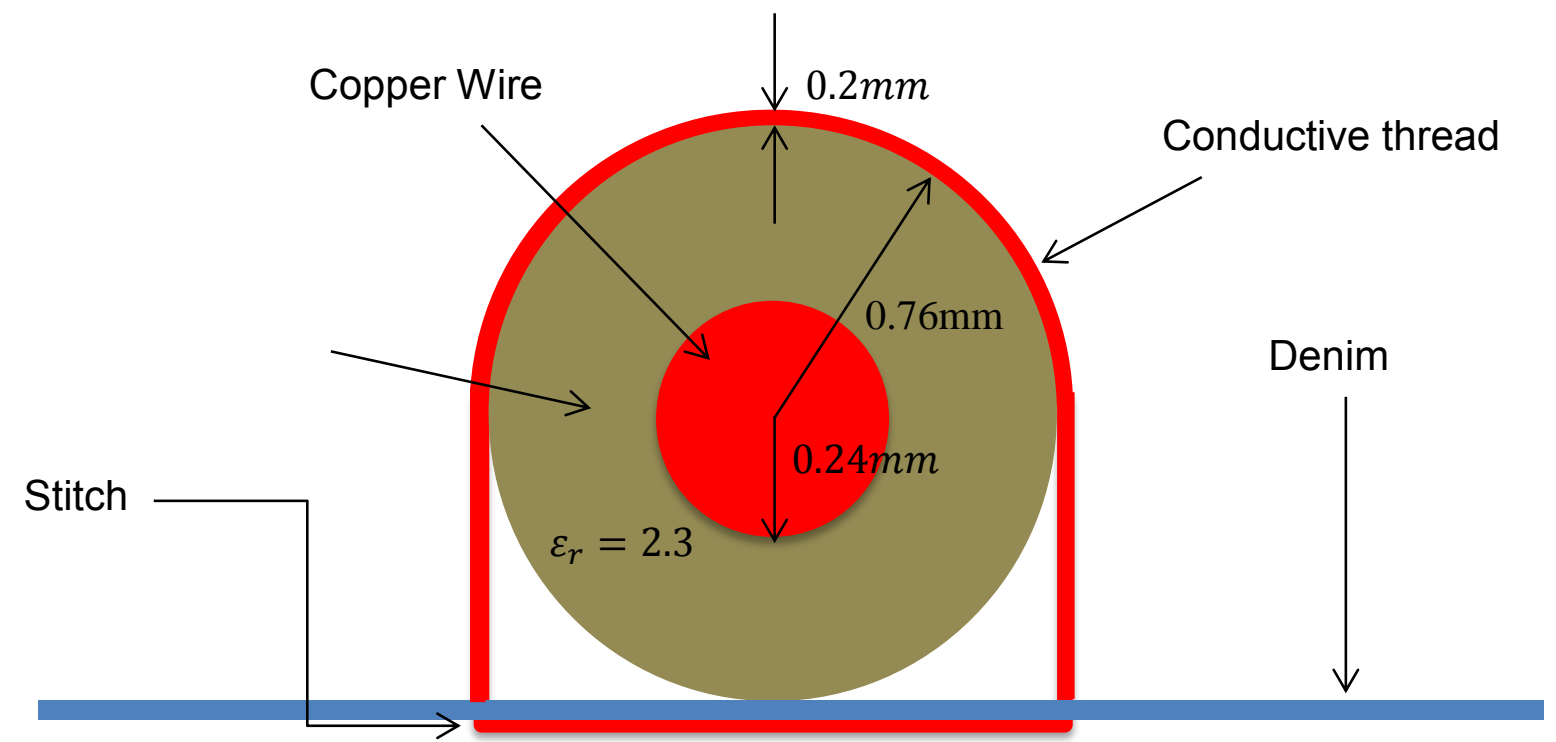

(a)

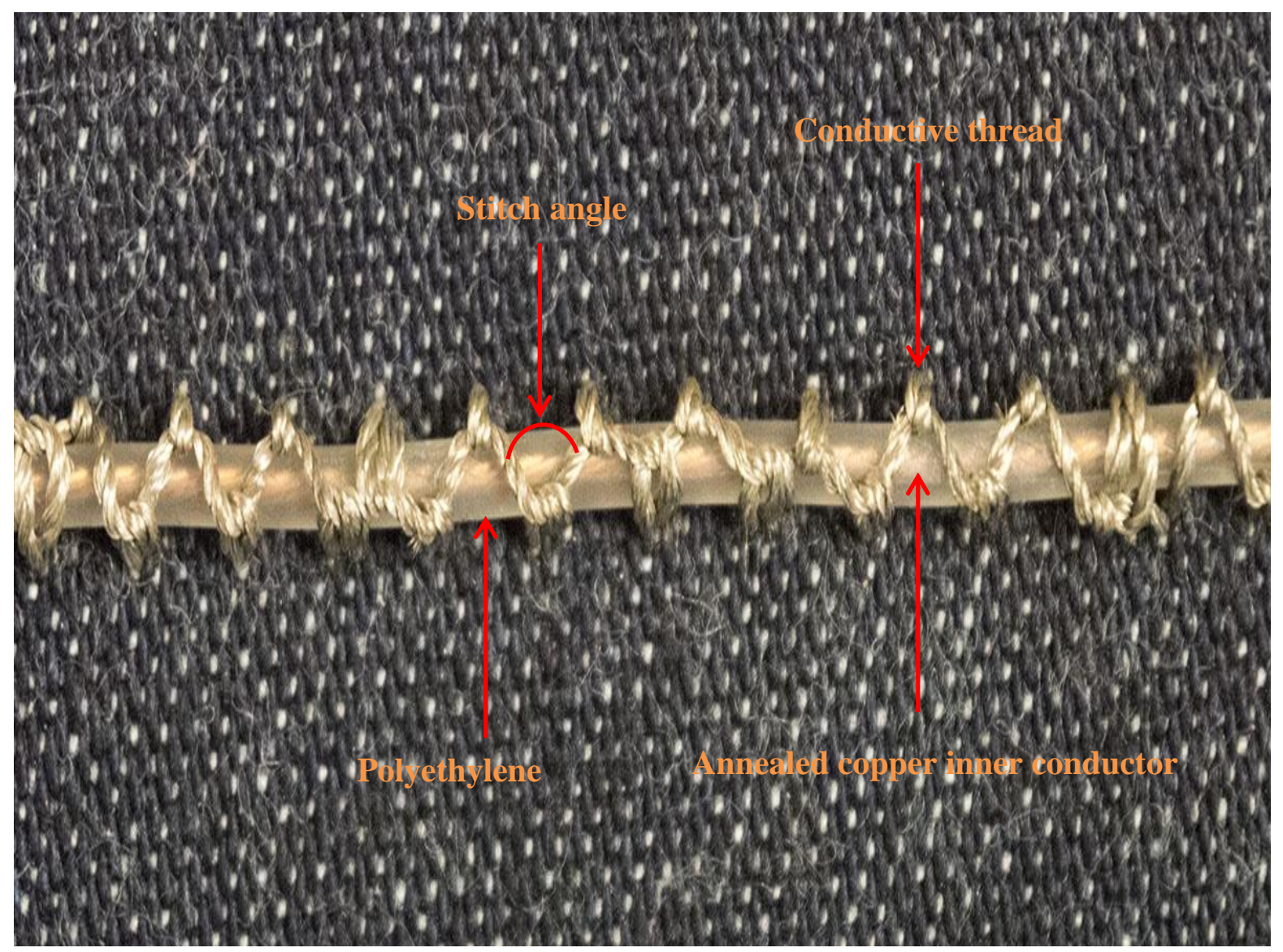

(b)

Figure 4.1 (a) Geometry of the stitched transmission line (b) Zoomed in view of fabricated stitched transmission line 
The design, as well as the fabricating process of the stitched transmission line, is very important in terms of its performance. Fabrication processes should guarantee good agreement with the design and simulation results. Additional results of the fabrication process should be the tolerance, robustness, and repeatability of the stitched transmission line. This arises from the fact that the stitched transmission line is supposed to be stitched on apparels and also operate under different circumstances e.g. weather conditions, body movements, temperature, bending and crumpling. Washability is another requirement of the stitched transmission line since it is stitched onto apparels.

To fabricate the stitched transmission line, an RG 174 braided coaxial cable was carefully stripped of its outer jacket and shield leaving behind the inner conductor and the tubular dielectric material made up of Polyethylene. The stripped transmission line was placed on a Denim material which serves as its substrate. A modified presser foot (see Fig.4.4) was fabricated to aid in stitching the conductive thread around the stripped RG174 and onto the Denim material with a Singer Talent ${ }^{\circledR}$ sewing machine with different stitch lengths while the stitch widths and tension were kept constant.

\subsubsection{Novel Presser Foot}

The presser foot is an accessory used with sewing machines to hold apparels in a regular position as it is fed through the machine and stitched. Sewing machines have feed dogs in the bed of the machine to provide traction and move the apparel as it is fed through the machine, while the sewer provides extra support for the apparel by guiding it with one hand. A presser foot helps in ensuring that the apparel is kept in check so that it does not rise and fall with the needle and pucker as it is stitched. When thick workpieces are to be sewn, such as quilts, a specialized accessory called a walking foot is often used rather than a presser foot. Presser's foot is typically spring-hinged to provide some flexibility as the textile moves beneath it. They have two toes which hold the fabric down on either side of the needle [4.13].

Because the stripped transmission line is placed on the apparel prior to lowering the presser foot on both the stripped transmission line and the apparel while running the stitch, there was a need to fabricate a presser foot that will meet this constraint without upsetting the tubular dielectric medium of the stripped transmission line. A novel presser foot for the 
sewing machine was fabricated as shown in Fig.4.2, while Fig. 4.3 depicts the presser foot been used in the construction of the stitched transmission line.

It is important to note here that the presser foot is designed to meet stitching requirements for a stripped RG174 braided coaxial cable. For a different application say for an RG58 braided coaxial cable, a different presser foot must be designed and necessary adjustments made to meet its stitching requirements. 
(a)
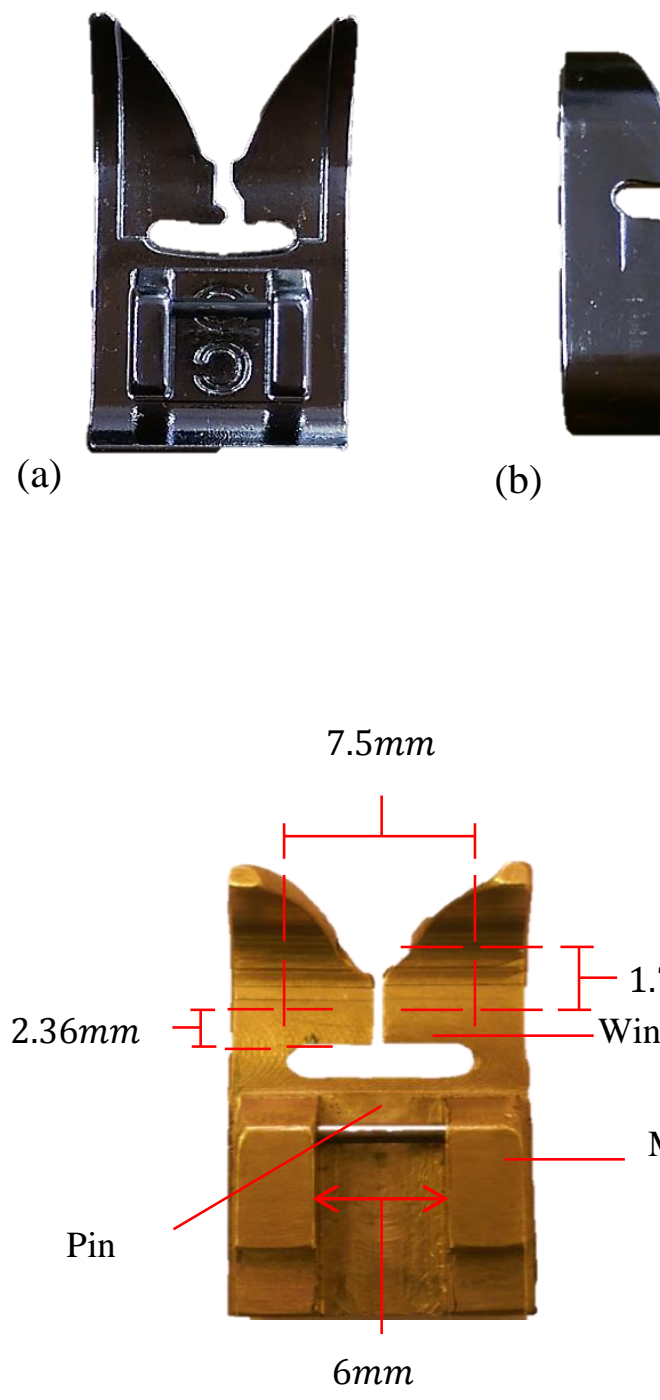

(e)

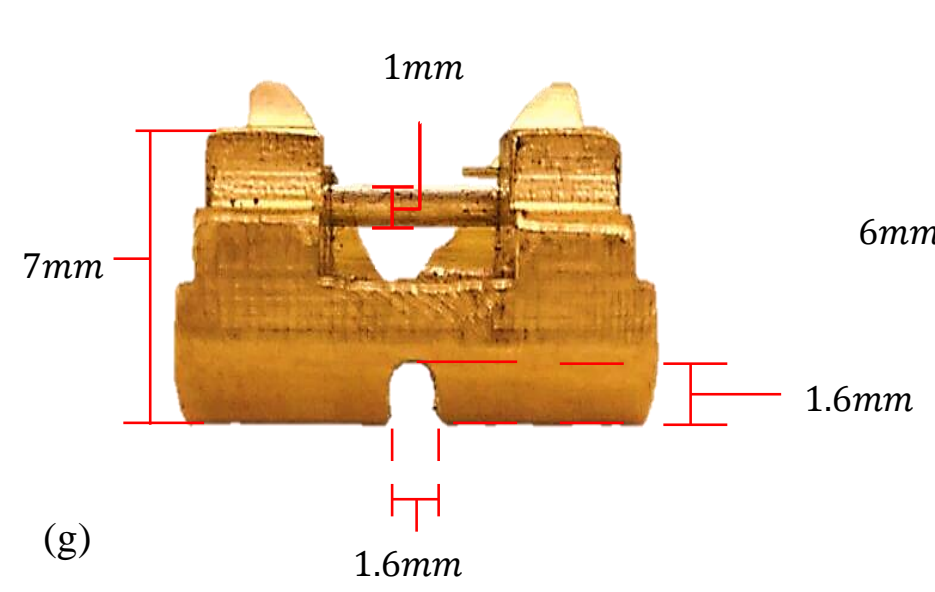

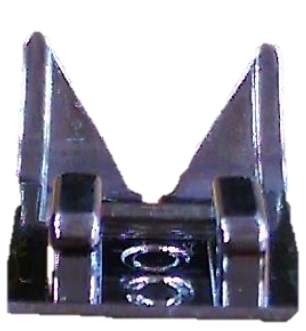

(c)

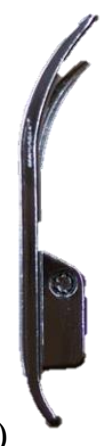

(d)

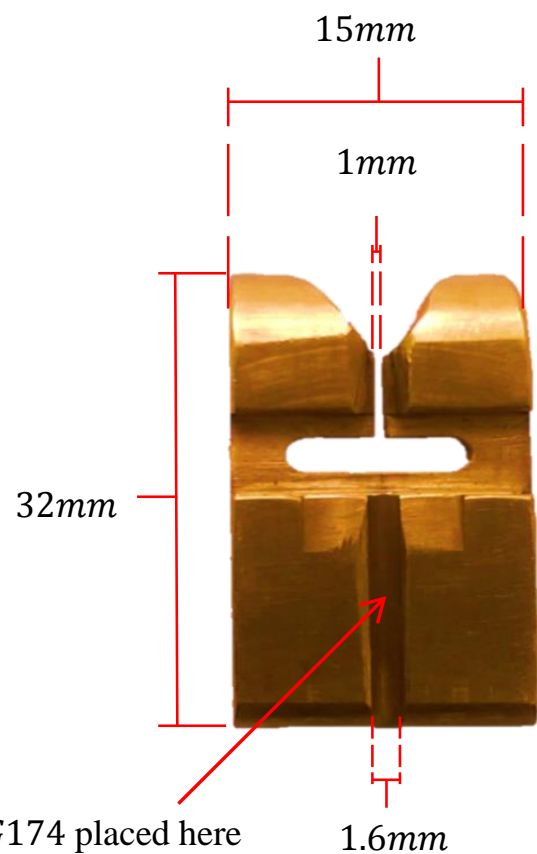

(f)

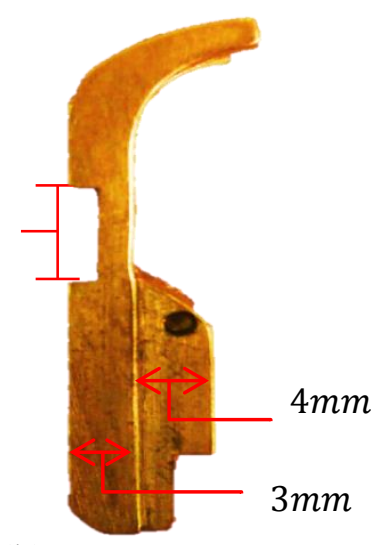

(h)

Figure 4.2 (a) - (d) and (e) - (h) shows the top, bottom, rear and side view of the Singer AllPurpose Snap-On Presser Foot and the novel presser foot respectively

Note: All dimensions in millimeters (not to scale) 


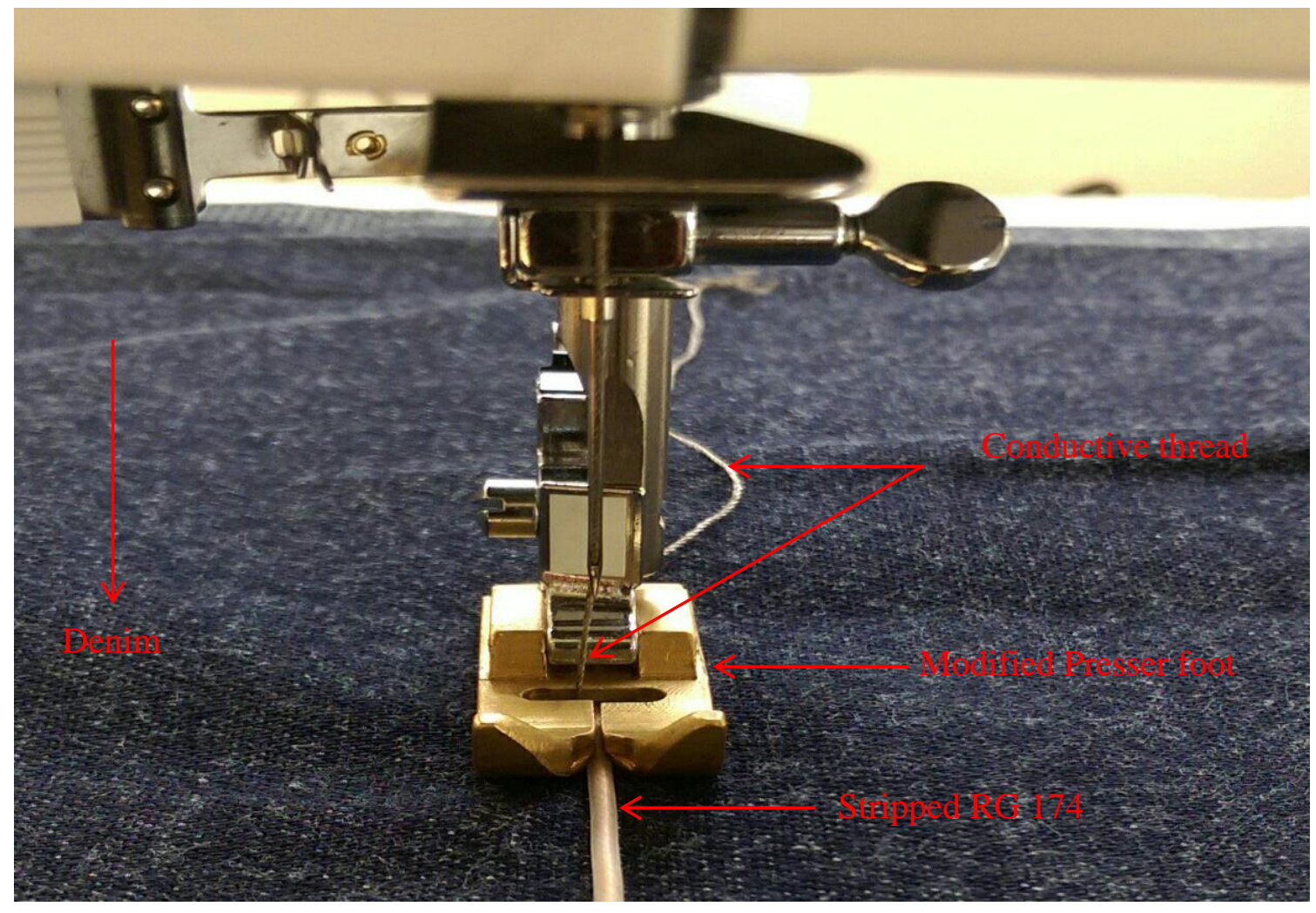

Figure 4.3 Novel presser foot used with Singer Talent sewing machine

\subsubsection{Stitch Angle}

For a braided shield with a typical braid pattern as shown in Fig. 4.4, the braid or weave angle which is the angle formed by the carriers with the longitudinal axis of the braided coaxial cable is given by

$\beta_{X}=\tan ^{-1} \frac{2 \pi D_{o} N_{W}}{C_{C}}$ 


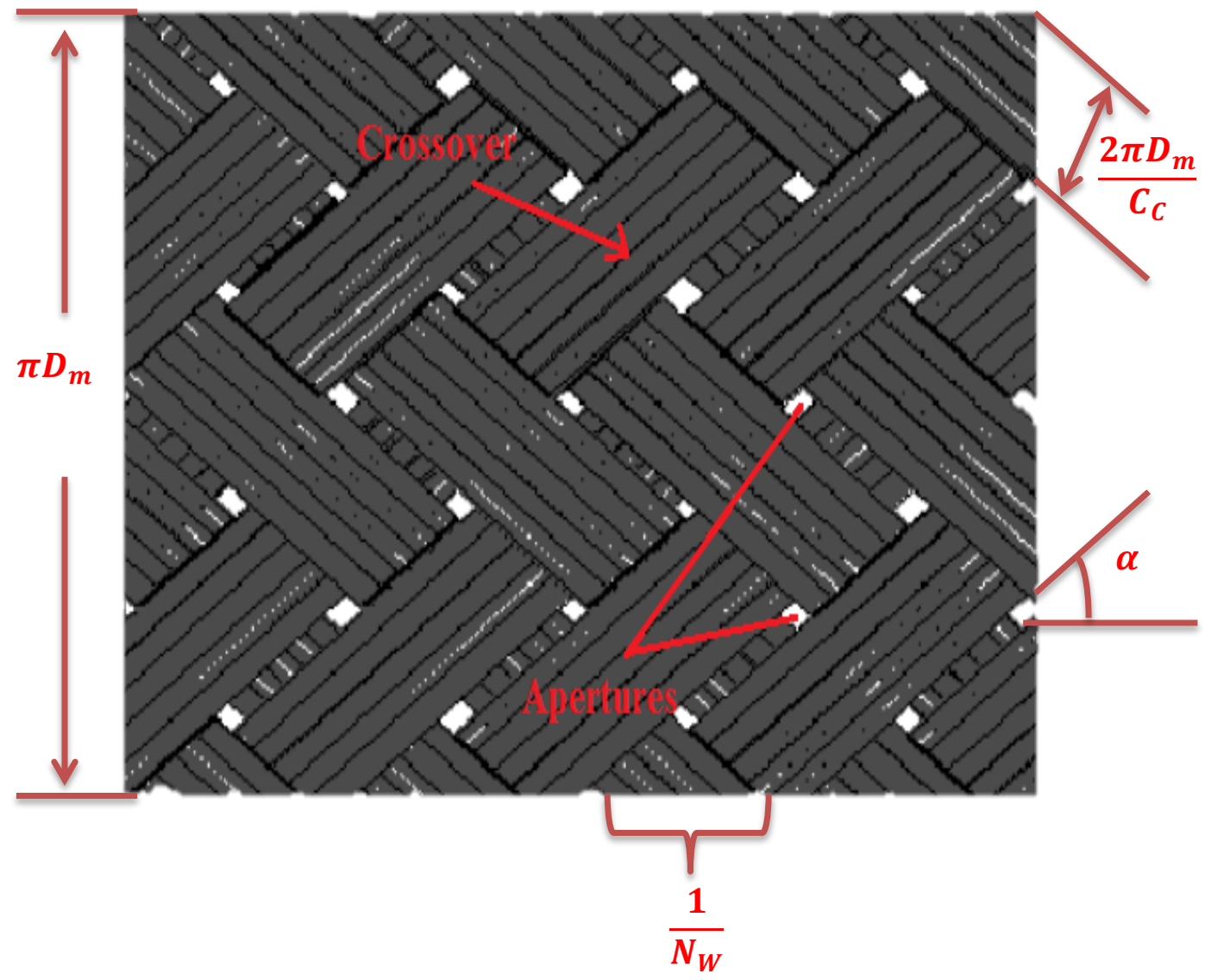

Figure 4.4 Typical braid patterns with braid angle $\beta$ by Vance [4.14]

Similarly, for the stitched transmission line, the stitch angle which is the angle formed by the carriers with the longitudinal axis of the stitched transmission line can be estimated using a section of the transmission line as shown in Fig.4.5. 


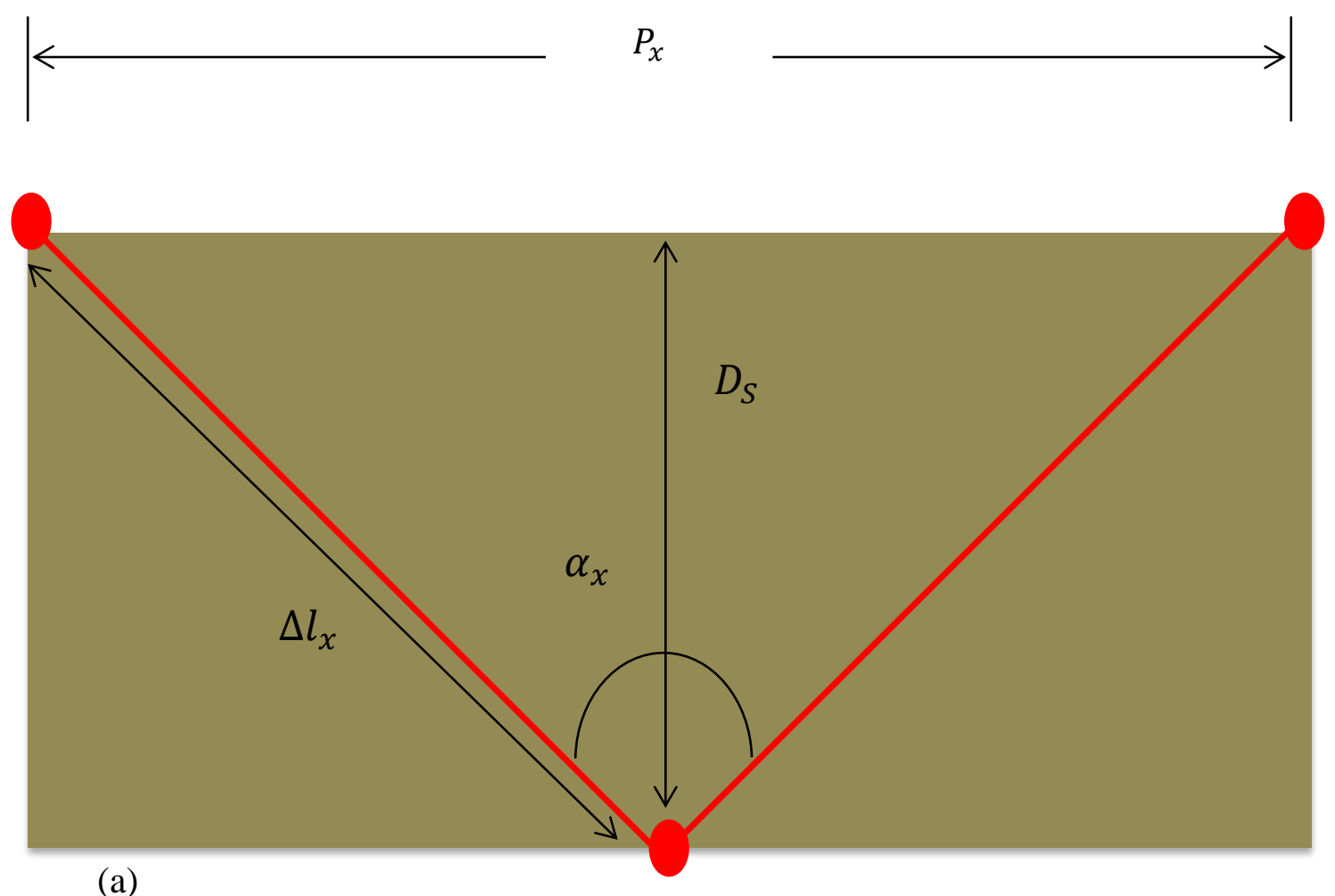

(a)
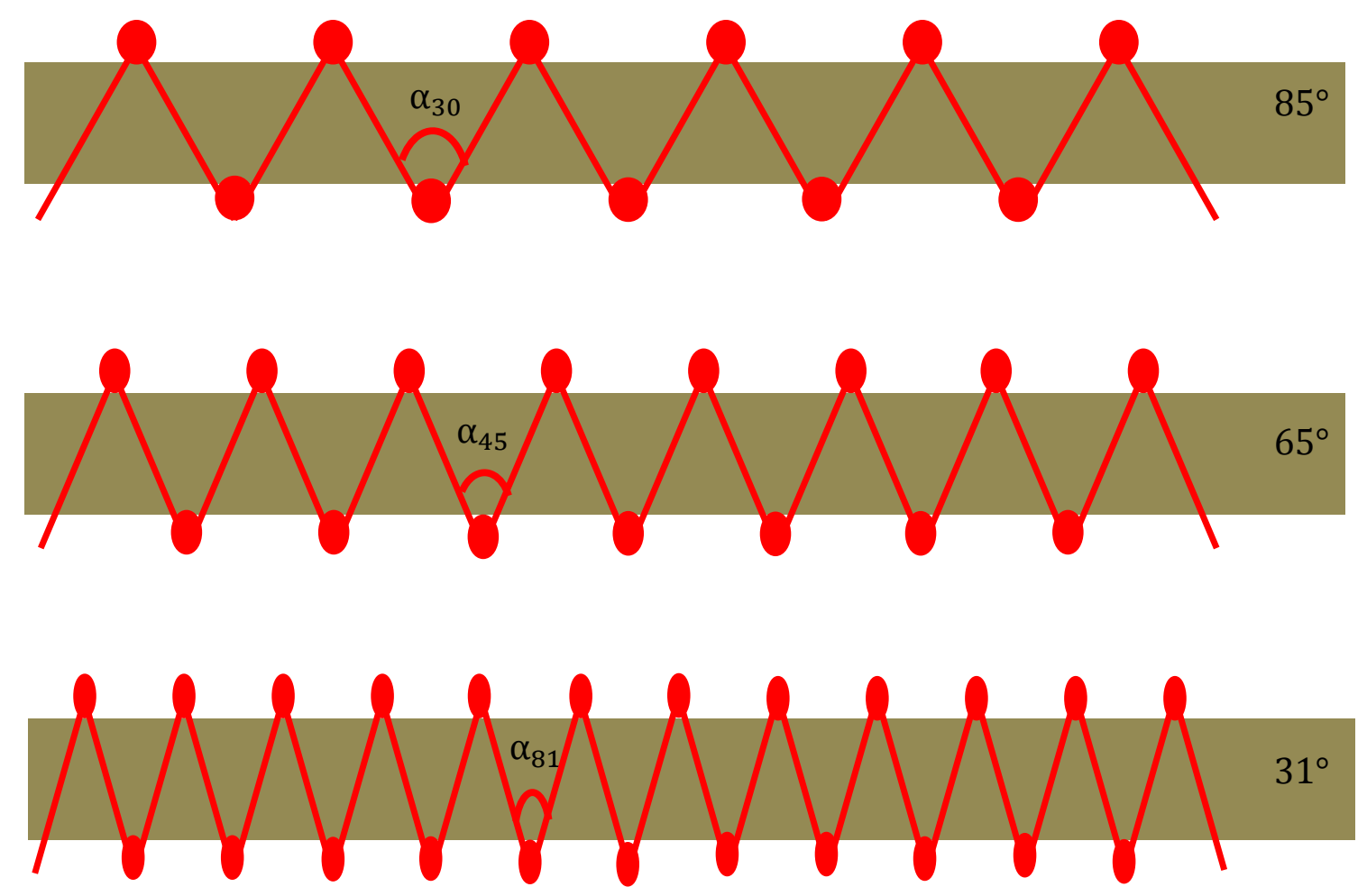

(b)

Figure 4.5 Schematic illustrations of (a) Stitch angle and (b) Three different stitch angles 
Here,

$\alpha_{x}=2 \tan ^{-1}\left(\frac{P_{x}}{2 D_{S}}\right)$

Where, $D_{S}=1.52 \mathrm{~mm}$ is the diameter of the stitched transmission line before stitching, $P_{x}=$ $P_{60}=2.76 \mathrm{~mm}, P_{90}=1.94 \mathrm{~mm}, P_{162}=0.83 \mathrm{~mm}$ is the pitch of the stitch with $x=$ $[60,90,162]$ signifying the number of stitches and $\Delta l_{x}$, the stitch length.

The stitched transmission line was fabricated with three different stitch angles, $\alpha_{30}=$ $85^{\circ}\left(\right.$ stitch legth $\left.\Delta l_{1.7 \mathrm{~mm}}\right), \alpha_{45}=65^{\circ}$ (stitch legth $\left.\Delta l_{1.5 \mathrm{~mm}}\right)$

and $\alpha_{81}=31^{\circ}$ (stitch legth $\left.\Delta l_{1.2 \mathrm{~mm}}\right)$. The stitch length which is determined by the feed dogs and stitch length regulator, determines the stitch angle of the stitched transmission line. The longer the stitch the bigger the stitch angle and vice versa. The stitch angle determines the level of coverage offered by the stitch transmission line and also plays a significant role in determining the shape of the stitch and its magnetic coupling as stated earlier in chapter three. Also, the more the shield coverage, the less the radiation of energy and ingres of RF signals to and from the stitched transmission line. However, this does not necessarily mean less signal attenuation.

For clarity and better understanding, stitch lengths will be used throughout the text in place of stitch angles except stated otherwise.

\subsection{Full Field Model with CST Microwave Studio Suite ${ }^{\circledR}$}

The stitched wearable transmission line was modeled as a composite counter-wound helical structure with a concentric conductor as proposed by Wait [4.15] as shown in Fig. 4.6. This consists of an inner conductor made up of annealed copper, surrounded by a polyethylene (PE) insulating layer, a double helix annealed copper shield and a denim substrate onto which the stitched transmission line is stitched.

The stitched transmission line design was carried out using CST Studio Suite ${ }^{\circledR}$, which is an electromagnetic simulation software that uses fast and memory efficient Finite Integration Technique (FIT) method and comprises of tools for the design and optimization of devices operating in a wide range of frequencies - static to optical. Analyses on CST Studio Suite ${ }^{\circledR}$ may include thermal and mechanical effects, as well as circuit simulation [4.16]. The 
designed stitched transmission line was carried out using the CST Microwave Studio ${ }^{\circledR}$ (CST MWS) as depicted in Fig.4.7. The cross section of the stitched wearable transmission line is shown in Fig. 4.8, while the design dimensions are presented in Table 4.1. To model the shield which is made up of two helices, the analytical curve was used which gives the option of having either the solid wire model to be natural or rounded.

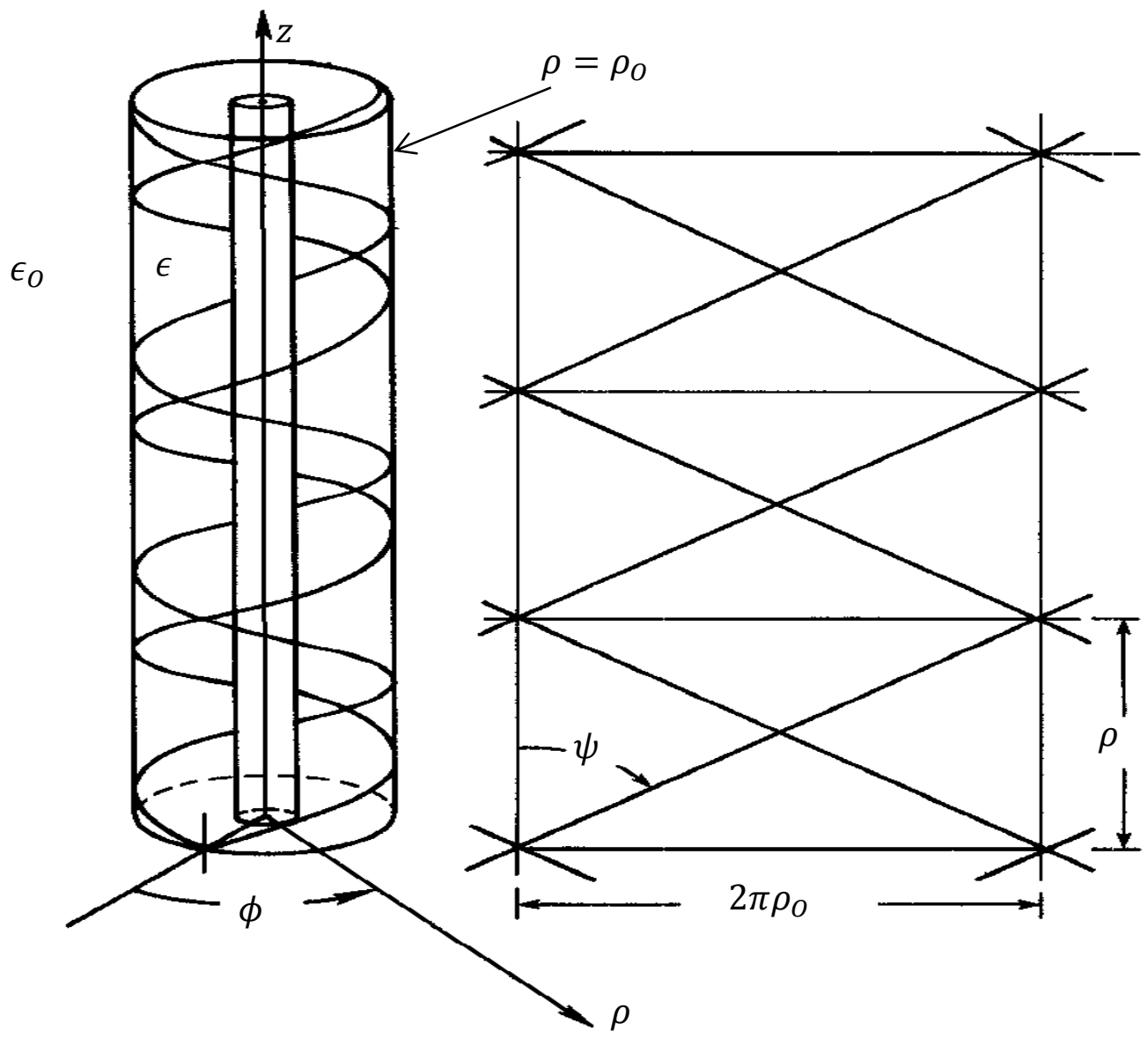

Figure 4.6 Perspective view of counter-wound helices and planar development of the cylindrical surface by Wait [4.15] 


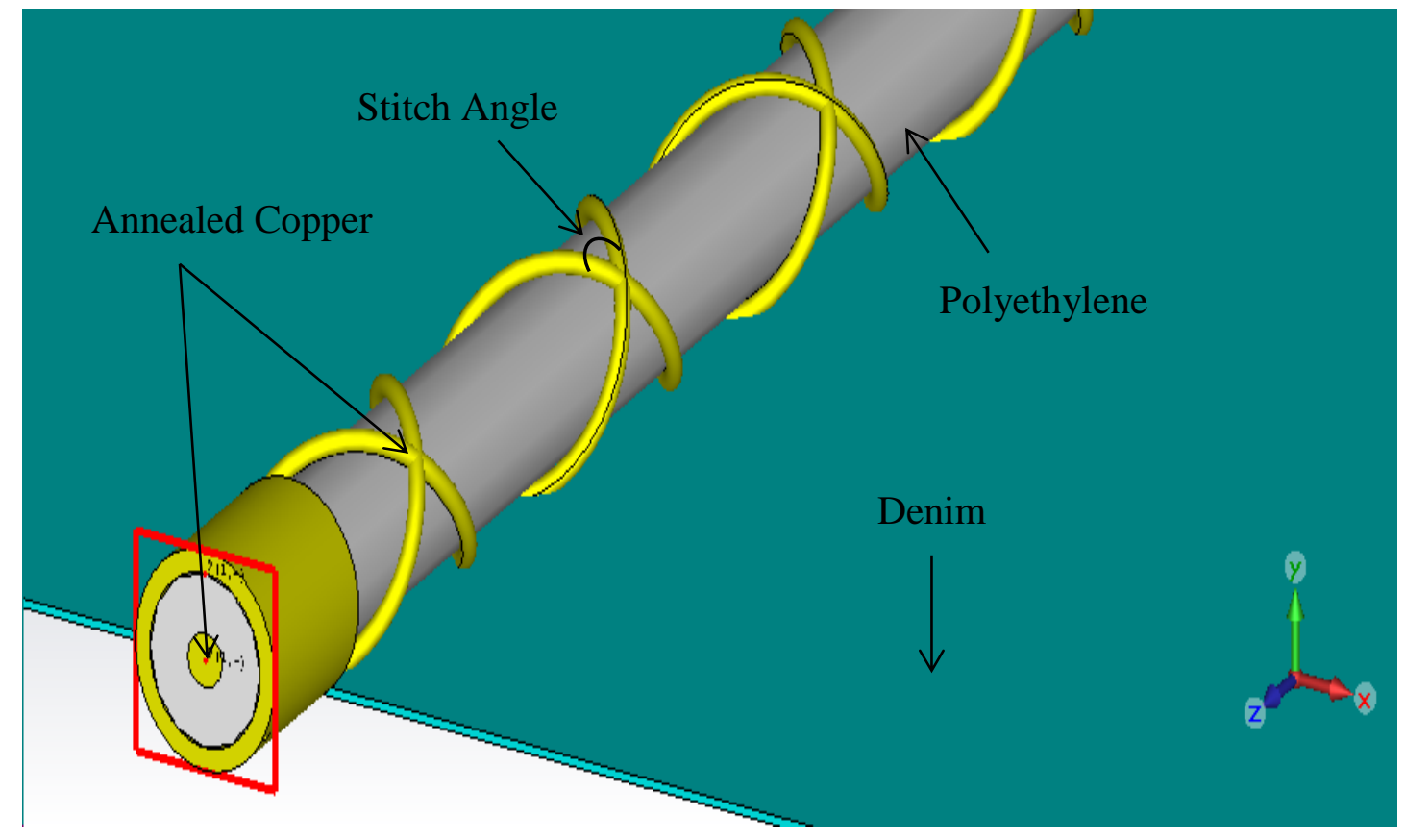

Figure 4.7 3-D view of stitched transmission line design with CST Microwave Studio suite ${ }^{\circledR}$

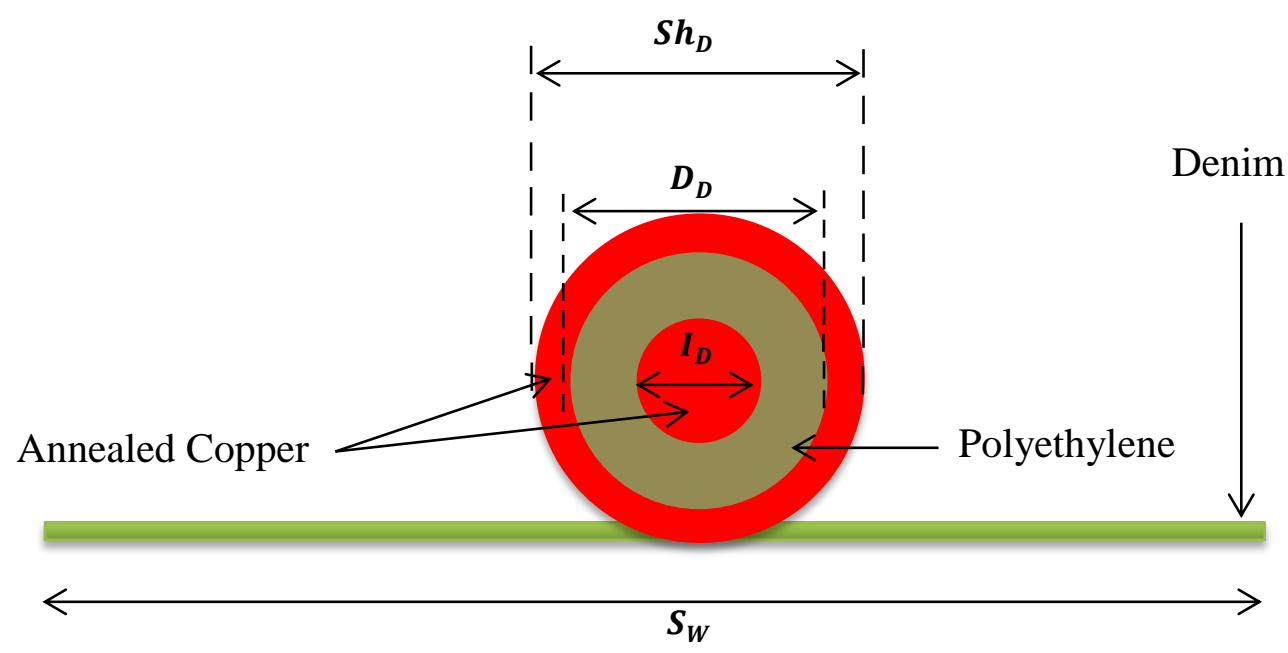

Figure 4.8 Cross section of the stitched transmission line 
Where $S h_{D}, D_{D}, I_{D}$ and $S_{D}$ are the shield diameter, dielectric diameter, inner conductor diameter and substrate diameter respectively.

TABLE 4.1 CST Microwave Studio Suite design dimensions with stitch angle at $85^{\circ}$

\begin{tabular}{|c|c|c|c|c|c|c|c|c|c|c|}
\hline S/NO & Name & $\begin{array}{c}\text { Outer } \\
\text { Radius }\end{array}$ & $\begin{array}{c}\text { Inner } \\
\text { Radius }\end{array}$ & $\begin{array}{c}\mathbf{X} \\
\text { Centre }\end{array}$ & $\begin{array}{c}\mathbf{Y} \\
\text { Centre }\end{array}$ & $Z_{\min }$ & $Z_{\max }$ & Segments & Component & Material \\
\hline 1 & Dielectric & 0.76 & 0.24 & 0 & 0 & 3 & 157 & 0 & 1 & $\begin{array}{l}\text { Polyethylene } \\
\text { (lossy) }\end{array}$ \\
\hline 2 & $\begin{array}{c}\text { Inner } \\
\text { Conductor }\end{array}$ & 0.24 & 0.0 & 0 & 0 & 3 & 157 & 0 & 1 & $\begin{array}{c}\text { Copper } \\
\text { (Annealed) }\end{array}$ \\
\hline 3 & Shield1 & 0.96 & 0.76 & 0 & 0 & 3 & 5 & 0 & 1 & $\begin{array}{c}\text { Copper } \\
\text { (Annealed) }\end{array}$ \\
\hline 4 & Shield2 & 0.96 & 0.76 & 0 & 0 & 155 & 157 & 0 & 1 & $\begin{array}{c}\text { Copper } \\
\text { (Annealed) }\end{array}$ \\
\hline 5 & Substrate & $\begin{array}{c}X_{\min } \\
-50 \\
\end{array}$ & $\begin{array}{c}X_{\max } \\
50 \\
\end{array}$ & $\begin{array}{r}Y_{\min } \\
-0.76 \\
\end{array}$ & $\begin{array}{r}Y_{\max } \\
-0.84 \\
\end{array}$ & $\begin{array}{c}\boldsymbol{Z}_{\min } \\
5\end{array}$ & $\begin{array}{c}Z_{\max } \\
155 \\
\end{array}$ & \multicolumn{2}{|c|}{ Component } & $\begin{array}{c}\text { Material } \\
\text { Denim }\end{array}$ \\
\hline
\end{tabular}

TABLE 4.2 Analytical curve of the helices

\begin{tabular}{cccccccc}
\hline S/NO & \multicolumn{4}{c}{ Analytical curve } & \multicolumn{2}{c}{ Parameter range } & Curve \\
\cline { 2 - 7 } & Analytical & $\mathrm{X}(\mathrm{t})$ & $\mathrm{Y}(\mathrm{t})$ & $\mathrm{Z}(\mathrm{t})$ & $\mathrm{Min}(\mathrm{t})$ & $\operatorname{Max}(\mathrm{t})$ \\
$\mathbf{1}$ & Analytical1 & $0.86 * \sin (200 * t)$ & $0.86 * \cos (200 * t)$ & $156.5 * t$ & 0.0197 & 1.002 & 1 \\
$\mathbf{2}$ & Analytical2 & $0.86 * \cos (200 * t)$ & $0.86 * \sin (200 * t)$ & $156.5 * t$ & 0.0197 & 1.002 & 2 \\
\hline
\end{tabular}

TABLE 4.3 Termination of the helices

\begin{tabular}{ccccccc}
\hline S/NO & Name & Folder & Radius & Modelling & Termination & Material \\
\hline $\mathbf{1}$ & Wire1 & Curve1 & 0.1 & Solid wire model & Natural & Copper (Annealed) \\
$\mathbf{2}$ & Wire2 & Curve2 & 0.1 & Solid wire model & Natural & Copper (Annealed) \\
\hline
\end{tabular}

The stitched transmission line design was carried out with three different stitch lengths of $1.7 \mathrm{~mm}, 1.5 \mathrm{~mm}$ and, $1.2 \mathrm{~mm}$, corresponding to stitch angles $31^{\circ}, 65^{\circ}$ and $85^{\circ}$. The scattering parameter results are as presented in Fig.4.9. For these simulations results, both $S_{11}$ which represent how much power is reflected back towards port 1 and $S_{21}$ which denotes the power transferred from port 1 to port 2 would be analysed. For instance if $s_{11}=0 \mathrm{~dB}$ all the power 
from port 1 is reflected back to port 1 , while for $S_{21}=0$ implies that all the power from port 1 ends up at the terminals of port 2.

From Fig. 4.9 the transmission coefficient, $S_{21}$, represents the power received at port 2 due to power input at port port 1 , while the reflection coeficient, $S_{11}$, indicates how much power is reflected back to port 1 compared to the power supplied to it.

The reflection coefficient $S_{11}$ for stitch angle $85^{\circ}$ are below $-3 d B$ in the entire operataion band, while the transmission coefficients $S_{21}$ are better than $-4.3 \mathrm{~dB}$ for frequencies up to $2.5 \mathrm{GHz}$. Equally the reflection coefficient $S_{11}$ for stitch angle $65^{\circ}$ are below $-2.8 \mathrm{~dB}$ in most of the operation band, and the transmission coefficients $S_{21}$ are better than $-3.3 d B$ for frequencies up to $2.16 \mathrm{GHz}$. Finally, reflection coefficient $S_{11}$ for stitch angle $31^{\circ}$ are below $-5 d B$ for frequencies up to $2.55 \mathrm{GHz}$ in most of the operation band, and the transmission coefficients $S_{21}$ are better than $-3 d B$ for frequencies up to $2.5 \mathrm{GHz}$.

Generally a transmission line with a better shield coverage tends to have less radiation loss as more shield coverage means less radiation of energy; however, this does not necessarily mean less signal attenuation. Radiation loss also tends to increase with an increase in frequency. Hence, at high frequencies, braided coaxial cables, for example, are doubled or tripled shielded to minimize losses due to radiation. In the case of the stitched transmission line, laminating it is an option.

For a loss free network, the total power exiting say $\mathrm{N}$ ports must be equal to the total incident power. That is the total power leaving the network must be accounted for in the power reflected from the incident port and the power transmitted. Thus

$P_{\text {incident }}=P_{\text {exit }}$

$\frac{P_{\text {reflected at port } 1}}{P_{\text {incident }}}+\frac{P_{\text {transmitted from port1-port } 2}}{P_{\text {incident }}}=1$

And since scattering parameters are squares of power ratios, Eqn.4.3c is also true for loss-free networks.

$S_{11}^{2}+S_{21}^{2}=1$

Here we apply proof by contradiction to show that the stitched transmission line is lossy by assuming that the stitched transmission line is loss free. 

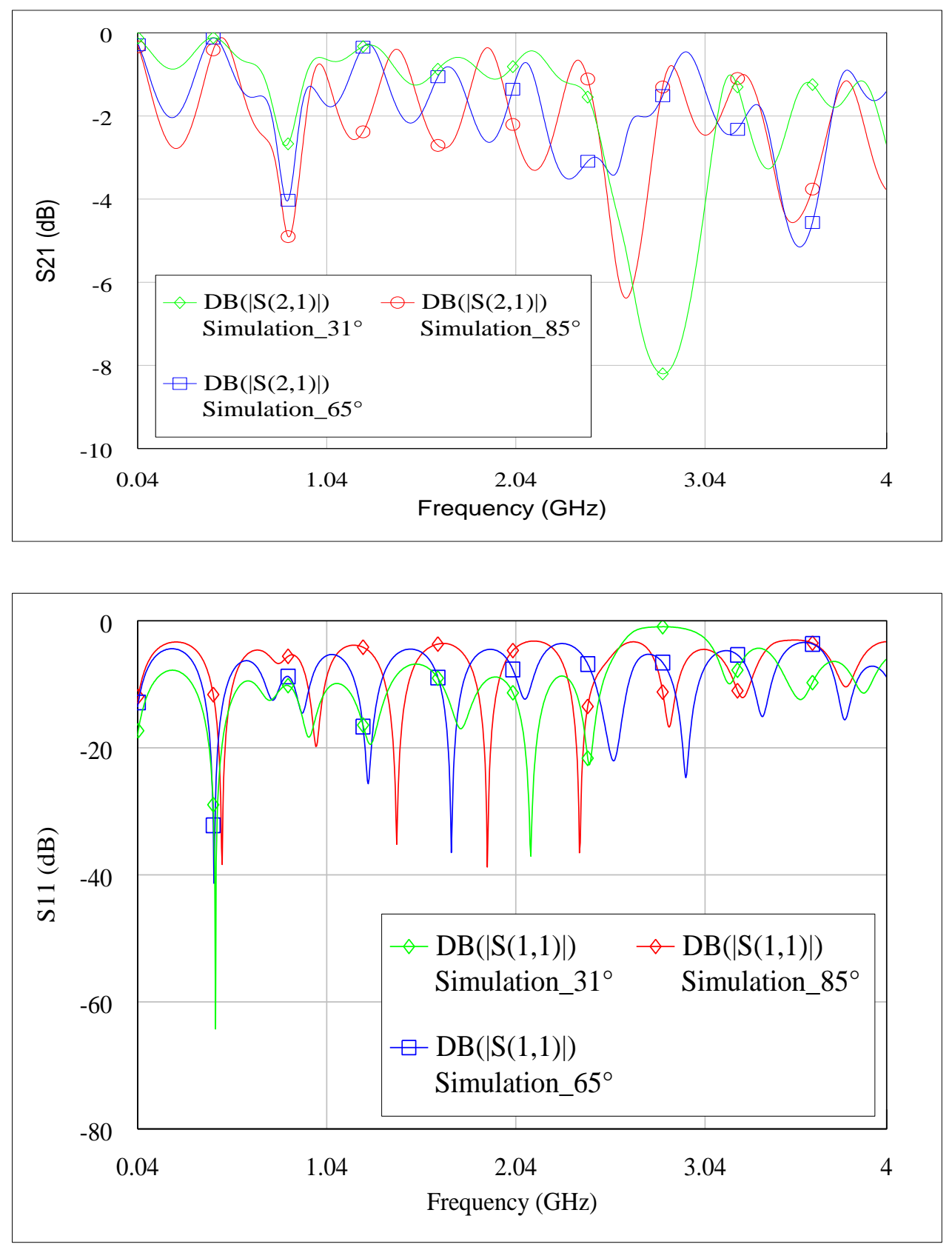

Figure 4.9 S-Parameters of stitched transmission line with stitch angles $85^{\circ}, 65^{\circ}$ and $31^{\circ}$ corresponding to stitch lengths $1.7 \mathrm{~mm}, 1.5 \mathrm{~mm}$ and, $1.2 \mathrm{~mm}$ respectively, using CST Microwave Studio Suite ${ }^{\circledR}$

To show that the stitched transmission line is not loss-free, a plot of $S_{11}^{2}+S_{21}^{2}$ against frequency is carried out as shown in Fig.4.10. Supposing the sum of the squares of the refection coeffiecient and transmission coefficent from the plot does not equal to one, then that proves that the transmission line is not loss free as earlier assumed. Form Fig. 4.10, it can be 
seen that the sum of squares of $S_{11}$ and $S_{21}$ is not equal to 1 throughout the plot, as such the transmission line is not loss free, which is also a confirmation of what was obtained in Fig. 4.9 .

For stitch angles of $85^{\circ}, 65^{\circ}$ and $31^{\circ}$, the stitched transmission line with stitch angle of $65^{\circ}$ appears to be lossier compared to stitch angles of $85^{\circ}$ and $31^{\circ}$. Though one will be quick to assume that the stitched transmission line with stitch angle of $85^{\circ}$ and $31^{\circ}$ are more lossy compared to the stitched trarnsmission line with stitch angle at $65^{\circ}$; because the stitched transmission line with stitch angle at $85^{\circ}$ has less DC resistance (less DC loss) and less shield coverage (more radiation loss) compared with the stitched transmission line with stitch angle at $31^{\circ}$, which has more DC resistance (more DC loss) and more shield coverage (less radiation loss), compared to the stitched transmission line with stitch angle at $65^{\circ}$ whose DC resistance (which translate to DC loss) and shield coverage (which translate to radiation loss) lies just inbetween the two. Here the radiation loss is dominant as a result of the poor shield coverage ascribed the stitched transmission line, as radiation loss is reduced when there is more shield coverage; conversely, the radiation loss tends to increase with increase in frequency. The radiation loss is seen to be higher at $0.8 \mathrm{GHZ}$ and $2.5 \mathrm{GHZ}$, with the transmission line behaving more like a leaky wave antenna and resonating at those frequencies. Furthermore as discussed in chapters two, there are other sources of loss that are associated with a transmission line apart from the conduction and radiation losses, and these include dielectric loss (which is same for the three stitched transmission lines), mismatch loss and induction loss. 


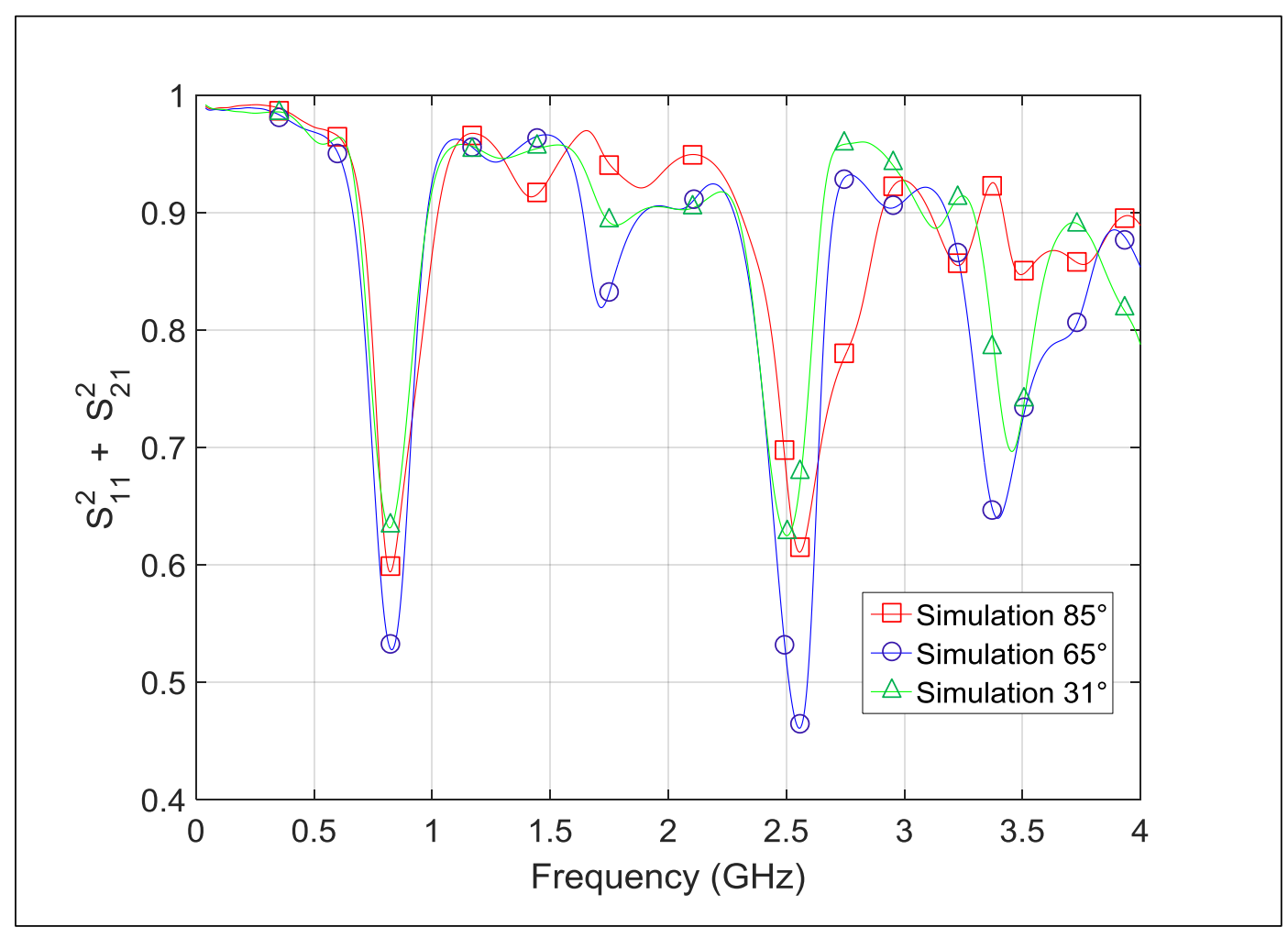

Figure 4.10 Plot of $\boldsymbol{S}_{\mathbf{1 1}}^{2}+\boldsymbol{S}_{\mathbf{2 1}}^{2}$ against frequency with stitch angles $85^{\circ}, 65^{\circ}$ and $31^{\circ}$

\subsubsection{Characteristic Impedance of the Stitched Transmission line}

Coaxial cable refers to cables with an inner conductor, an insulating medium and an outer conductor (also known as the shield), with all three sharing a common axis. Approximations for computing characteristic impedance of coaxial cables in which the inner conductor is circular, square, elliptical or rectangular and the other conductor is circular and noncircular has been presented in [4.17]-[4.26]. A couple of these are presented in Fig. 4.7, with their characteristic impedance given.

For the square coaxial line with an accuracy of within $0.5 \%$ for $t / b \leq 0.5$,

$$
Z_{0} \sqrt{k}=136.7 \log _{10}(0.9259 \mathrm{~b} / \mathrm{t})
$$

For the general rectangular coaxial line,

$$
Z_{0} \sqrt{k}=59.952 \ln \left(\frac{1+W^{I} / b}{W / b+t / b}\right)
$$


For the coaxial stripline where, $k$, is the dielectric constant of the medium filling the line interior,

$Z_{0} \sqrt{k}=94.172 \frac{K^{I}(x)}{K(x)}$

Where $x=\frac{2(W / b)}{1+(W / b)^{2}}$

For the elliptical coaxial line whose conductors are confocal ellipses with major axis $W^{I}$ and $W$, and minor axis $b$ and $t$ respectively, where $k$ is the dielectric constant of the medium filling the line interior,

$Z_{0} \sqrt{k}=59.952 \cosh ^{-1} W^{I} / W$

For the coaxial line with outer conductor of elliptical cross section,

$Z_{0}=59.952 \ln \left[G+\sqrt{\left(G^{2}-1\right)}\right]$

Where,

$$
\begin{gathered}
G=\frac{1}{2}\left\{\frac{2 r}{\left|\alpha_{0}\right| R}+\frac{\left|\alpha_{0}\right| R}{r}\left[1-E_{1}(r / R)\right]\left[1-\frac{E_{1}(r / R)}{2}\right]\right\} \\
E_{1}(r / R)=\left(1-\frac{1}{\left|\alpha_{0}\right|}\right)(r / R)^{F_{1}(r / R)} \\
F_{1}(r / R)=\frac{2}{\left|\alpha_{0}\right|}\left[1-\left(\frac{r}{R}\right)^{10 /\left|\alpha_{0}\right|}\right] \\
\alpha_{0}=\sqrt{1+\lambda}\left(1-\frac{\lambda^{2}}{8}+\frac{3}{128} \lambda^{4}\right)
\end{gathered}
$$

[4.26]

For the polygonal coaxial line,

$Z_{0} \sqrt{k}=\frac{188.344(b / d-1)}{N\{\tan (\pi / N)+(b / d-1) \chi\}}$ 
Where, $\quad \chi=\frac{1}{\pi}\left\{\psi\left(\frac{N+2}{2 N}\right)-\psi\left(\frac{1}{2}\right)\right\}, \psi(x)=-\gamma+\sum_{N=1}^{\infty}\left\{\frac{X-1}{N(N+X-1)}\right\}, \gamma=0.5772 \quad$ (Euler's constant), $N$, is the number of sides to the polygon, $b$, is the diameter of the inscribed circle of outer polygon and, $d$, is the diameter of the inscribed circle of inner polygon [4.17].

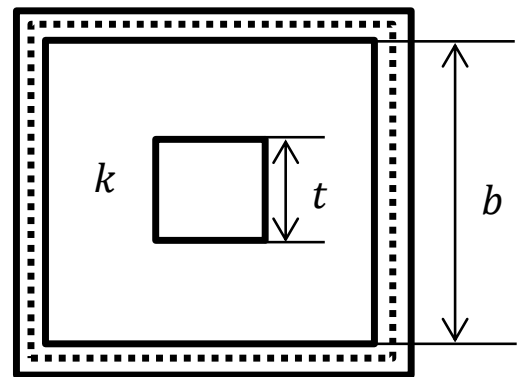

(a)

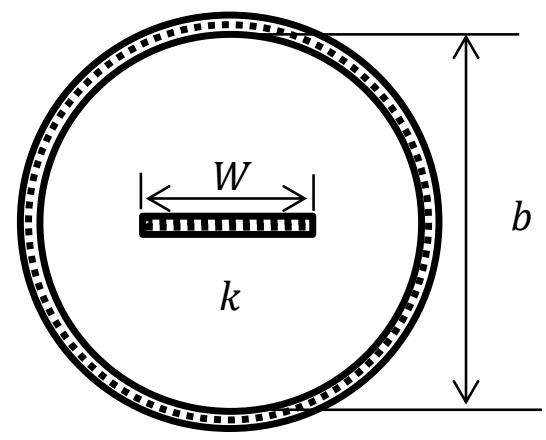

(c)

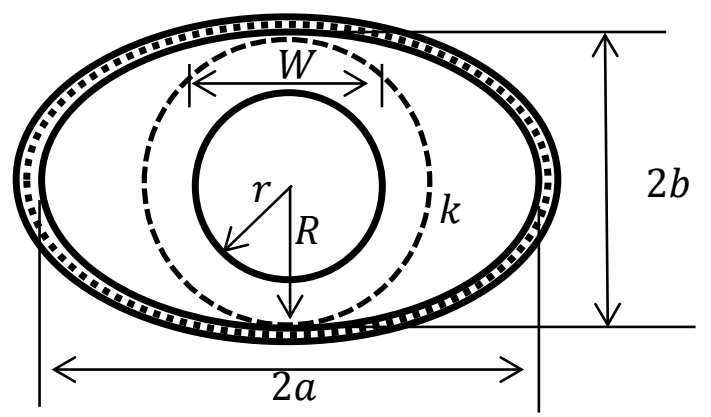

(e)

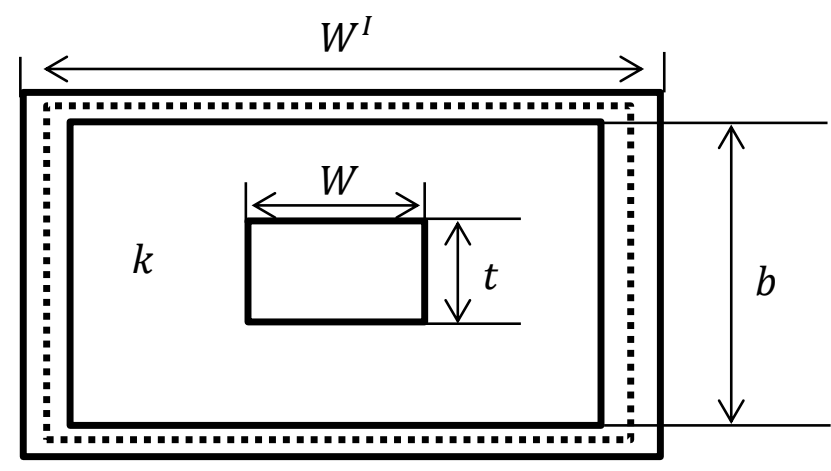

(b)

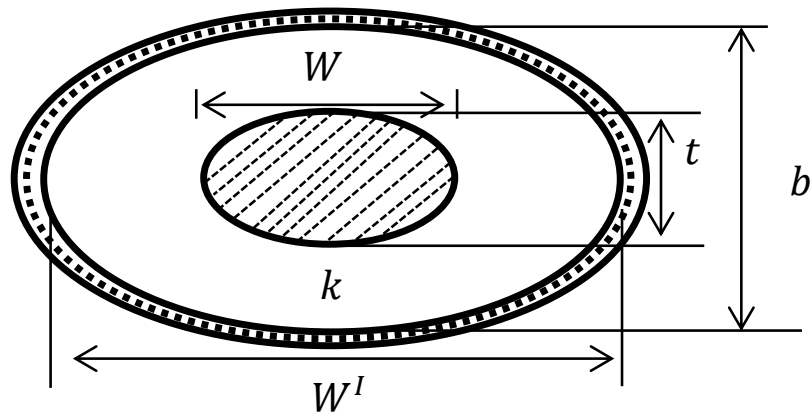

(d)

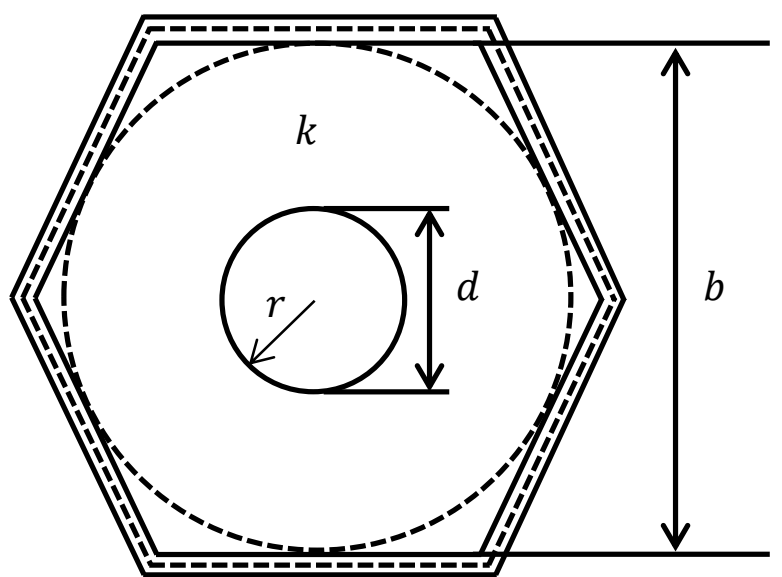

(f)

Figure 4.11 Cross section of (a) Square coaxial line (b) General rectangular coaxial line (c) Coaxial stripline (d) Elliptical coaxial line whose conductors are confocal ellipses (e) Coaxial line with outer conductor of elliptical cross section (f) Polygonal coaxial line with cylindrical centre conductor [4.17] and [4.26] 
To simplify analysis and fabrication, circular cross-sections are consistent for coaxial cables. In practical coaxial cables, the characteristic impedance is determined by cable geometry that is by the size and spacing between the conductors and the dielectric material between them. The cable length has no effect on its characteristic impedance. The shield which forms the outer conductor of coaxial cable acts to first keep the desired currents inside the coaxial cable, secondly to keep the undesired currents outside of the coaxial cable and lastly to provide a path to earth ground for external or interfering field on the coaxial cable. However, it is also important to note here that at high frequencies a good proportion of the power travels in the fields between the inner and outer conductors.

The following values are used at $f=4 \times 10^{9} \mathrm{GHz}$ for the stitched transmission line modelled as a composite counterwound helical structure [4.15] with a concentric conductor with CST Microwave Studio Suite ${ }^{\circledR}$ :

$$
\begin{gathered}
a=0.48 \mathrm{~mm}, b=1.52 \mathrm{~mm}, \mu_{0}=4 \pi \times 10^{-7} \mathrm{~N}^{-2}, \mu_{r}=1, \varepsilon_{0}=8.85 \times 10^{-12} \mathrm{Fm}^{-1} \\
\varepsilon_{r}=2.3, \sigma_{\text {diel }}=1 \times 10^{-13} \mathrm{Sm}^{-1}, \sigma_{\text {con }}=6.0 \times 10^{7} \mathrm{Sm}^{-1}, \delta=1.03 \mu \mathrm{m} .
\end{gathered}
$$

Where $a, b, \mu_{0,} \mu_{r}, \varepsilon_{0}, \varepsilon_{r}, \sigma_{c o n}$ and $\delta$ are the inner cylindrical conductor radius, outer conductor radius, permeability the of free space, relative permeability, permittivity the of free space, relative permittivity, conductivity the of the dielectric, conductivity of copper and skin depth of copper at $4 \mathrm{GHz}$.

The following parameters can also be computed as follows:

Series resistance per unit length,

$$
\begin{aligned}
& \boldsymbol{R}=\frac{R_{S}}{2 \pi}\left(\frac{1}{a}+\frac{1}{b}\right) \\
& \boldsymbol{R}=\frac{1}{2 \pi \sigma_{\operatorname{con} \delta} \delta}\left(\frac{1}{a}+\frac{1}{b}\right)
\end{aligned}
$$

Where $R_{S}=\frac{1}{\sigma_{c o n} \delta}$

$$
\boldsymbol{R}=7.078 \approx 7 \Omega . m^{-1}
$$

Series inductance per unit length, 
$\boldsymbol{L}=\frac{\mu_{0} \mu_{r}}{2 \pi} \ln \frac{b}{a}$

$$
\boldsymbol{L}=230.5 \mathrm{nH} \cdot \mathrm{m}^{-1}
$$

Shunt capacitance per unit length,

$C=\frac{2 \pi \varepsilon}{\ln \frac{b}{a}}$

Where $\varepsilon=\varepsilon_{0} \varepsilon_{r}$

$$
\boldsymbol{C} \approx 111 p F \cdot m^{-1}
$$

Shunt conductance per unit length

$$
\boldsymbol{G}=\frac{2 \pi \sigma_{\text {diel }}}{\ln \frac{b}{a}}
$$

$$
\boldsymbol{G} \approx 5.45 \times 10^{-13} \mathrm{Sm}^{-1}
$$

The characteristic impedance $Z_{0}$ of the line is given by:

$$
Z_{0}=\sqrt{\frac{R+j \omega L}{G+j \omega C}}
$$

$$
Z_{0} \approx(45.569-i 0.028) \Omega
$$

The propagation constant is given by:

$\gamma=\alpha+j \beta=\sqrt{(R+j \omega L) \cdot(G+j \omega C)}$

$\alpha=\frac{\sqrt{L C}}{2} \cdot\left[\left(\frac{R}{L}+1\right) \cdot\left(\frac{G}{C}+1\right)\right]$

This can be approximated such that

$\alpha \approx \frac{1}{2}\left(\frac{R}{z_{0}}+G Z_{0}\right)$

Which can be further separated as follows: 
$\alpha_{C}=\frac{R}{2 Z_{0}}$

$\alpha_{D}=\frac{G}{2 Z_{0}}$

Eqn.4.11 is the metal loss due to skin depth while Eqn. 4.12 which is due to dielectric losses can be further separated into loss due to dielectric loss tangent and loss due to substrate conductivity [4.29]. Hence,

$$
\begin{gathered}
\alpha_{C}=\frac{7.078}{2 \times 45.569}=0.07766 \\
\alpha_{D}=\frac{5.45 \times 10^{-13}}{2 \times 45.569}=5.9799 \times 10^{-15} \\
\alpha=0.07766+5.9799 \times 10^{-15} \approx 0.078 \mathrm{~Np} . \mathrm{m}^{-1}
\end{gathered}
$$

In $d B$,

$$
\begin{gathered}
\propto=8.686 \times \text { Attenuation in Np. } \mathrm{m}^{-1} \\
\propto=8.686 \times 0.078 \mathrm{~Np} . \mathrm{m}^{-1} \cong 0.68 \mathrm{~dB} . \mathrm{m}^{-1}
\end{gathered}
$$

$\beta=\omega \sqrt{L C} \cdot\left[1+\left(\frac{R G}{4 \omega^{2} L C}\right)\right]$

This can also be approximated as

$$
\begin{gathered}
\beta \approx \omega \sqrt{\boldsymbol{L C}} \\
\beta \approx 127 \mathrm{rad} . \mathrm{m}^{-1}
\end{gathered}
$$

Thus, the propagation constant, $\gamma$, is given as:

$$
\gamma=0.078+j 127
$$

Numerical modeling results with CST indicate characteristic impedance of $45.5 \Omega, 45.4 \Omega$ and $44.9 \Omega$ for stitch angles of $85^{\circ}, 65^{\circ}$ and $31^{\circ}$ respectively. This is seen to be quite close to the computed result of $Z_{0} \approx 46 \Omega \angle-0.034^{\circ}$. 
Furthermore, the characteristic impedance of the stitched transmission line will be determined using the reflection coefficient $S_{11}$ and transmission coefficient $S_{21}$ obtained from scattering parameter plots in Fig.4.9 for the three stitched angles $85^{\circ}, 65^{\circ}$ and $31^{\circ}$ at $1.1686 \mathrm{GHz}, 1.034 \mathrm{GHz}$ and $1.0973 \mathrm{GHz}$ respectively and results compared with the analytical result and numerical modelling results from CST Microwave Studio Suite ${ }^{\circledR}$

a. The circuit representation of the stitched transmission line is given by Fig. 4.12.

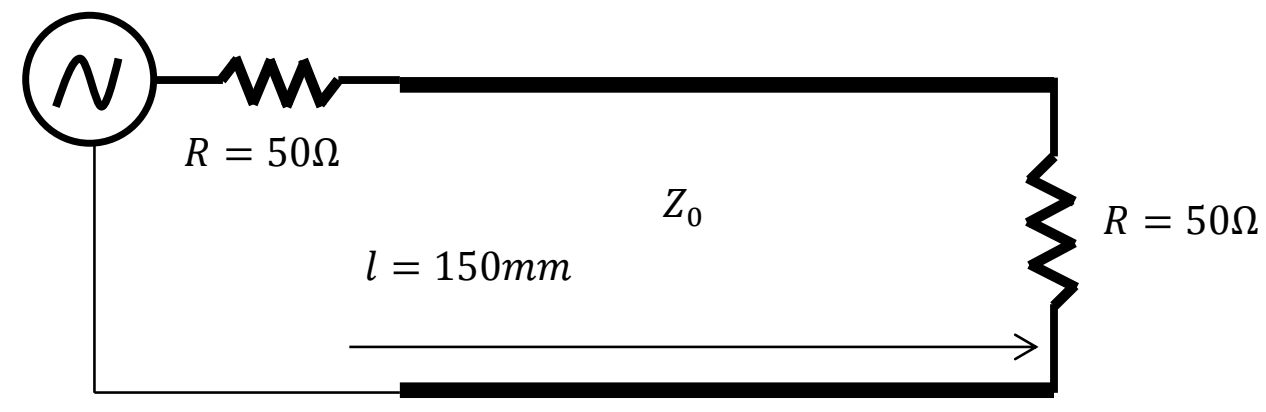

Figure 4.12 Circuit representation of the lossy stitched transmission line

Using,

$S_{11}=\frac{Z_{\text {in }}-Z_{0}}{Z_{\text {in }}+Z_{0}}$

The input impedance of the stitched transmission line with stitch angle at $85^{\circ}$ based on the peak $S_{11}$ at $1.1686 G \mathrm{~Hz}$ from Fig. 4.9, is given as:

$$
\begin{gathered}
S_{11}=\frac{Z_{\text {in }}-50}{Z_{\text {in }}+50}=0.6408 \\
Z_{\text {in }} \cong 228 \Omega
\end{gathered}
$$

For a lossy line,

$Z_{\text {in }}=Z_{0} \frac{Z_{L}+Z_{0} \tanh (\gamma(-l))}{Z_{0}+Z_{L} \tanh (\gamma(-l))}$

Where 


$$
\gamma=\alpha+j \beta
$$

The attenuation constant can be estimated directly from the insertion loss where,

$$
\begin{gathered}
S_{21} \approx e^{-\propto l} \\
0.7462=e^{-\propto\left(150 \times 10^{-3}\right)}
\end{gathered}
$$

Thus,

$$
\propto=1.9517 N p \cdot m^{-1}
$$

$$
\begin{gathered}
\alpha(-l)=\left(1.9517 \times\left(-150 \times 10^{-3}\right)+j 8.35\right) \\
\alpha(-l)=-0.29 N p
\end{gathered}
$$

Here, $\beta l$ is determined from Fig. 4.13 , where $\beta l$ is given by:

$$
\beta l=8.35 \mathrm{rad}
$$

Thus the propagation constant is finally given as,

$$
\gamma(-l)=-0.29+j 8.35
$$

Similarly, the difference in this values compared to the computed values can be attributed to the use of values from the s-parameter, unlike the computed values where line parameters were used.

Using,

$$
Z_{\text {in }}=Z_{0} \frac{Z_{L}+Z_{0} \tanh (\gamma(-l))}{Z_{0}+Z_{L} \tanh (\gamma(-l))}
$$

Solving for $Z_{0}$, using the quadratic formula gives:

$Z_{0}=\frac{\frac{-\left(z_{L}-Z_{\text {in }}\right)}{\tanh (\gamma(-l))} \pm \sqrt{\left(\frac{\left(z_{L}-Z_{\text {in }}\right)}{\tanh (\gamma(-l))}\right)^{2}+4 Z_{i n} Z_{L}}}{2}$

With $Z_{0}$ having two roots given by:

$$
Z_{01}=(71.61+j 29.93) \Omega
$$


Or

$$
Z_{02}=\left(-1.36 \times 10^{2}+j 5.67 \times 10^{1}\right) \Omega
$$

Here $Z_{01}=(72.16-j 32.28) \Omega$ is chosen as the characteristic impedance of the stitched transmission line. A choice of $Z_{02}=\left(-1.36 \times 10^{2}+j 5.67 \times 10^{1}\right) \Omega$ will translate to energy been generated within the transmission line that is proportional to the square of the impressed voltage or current. Thus this indicates that the transmission line contributes power to itself, which is not a feasible result.

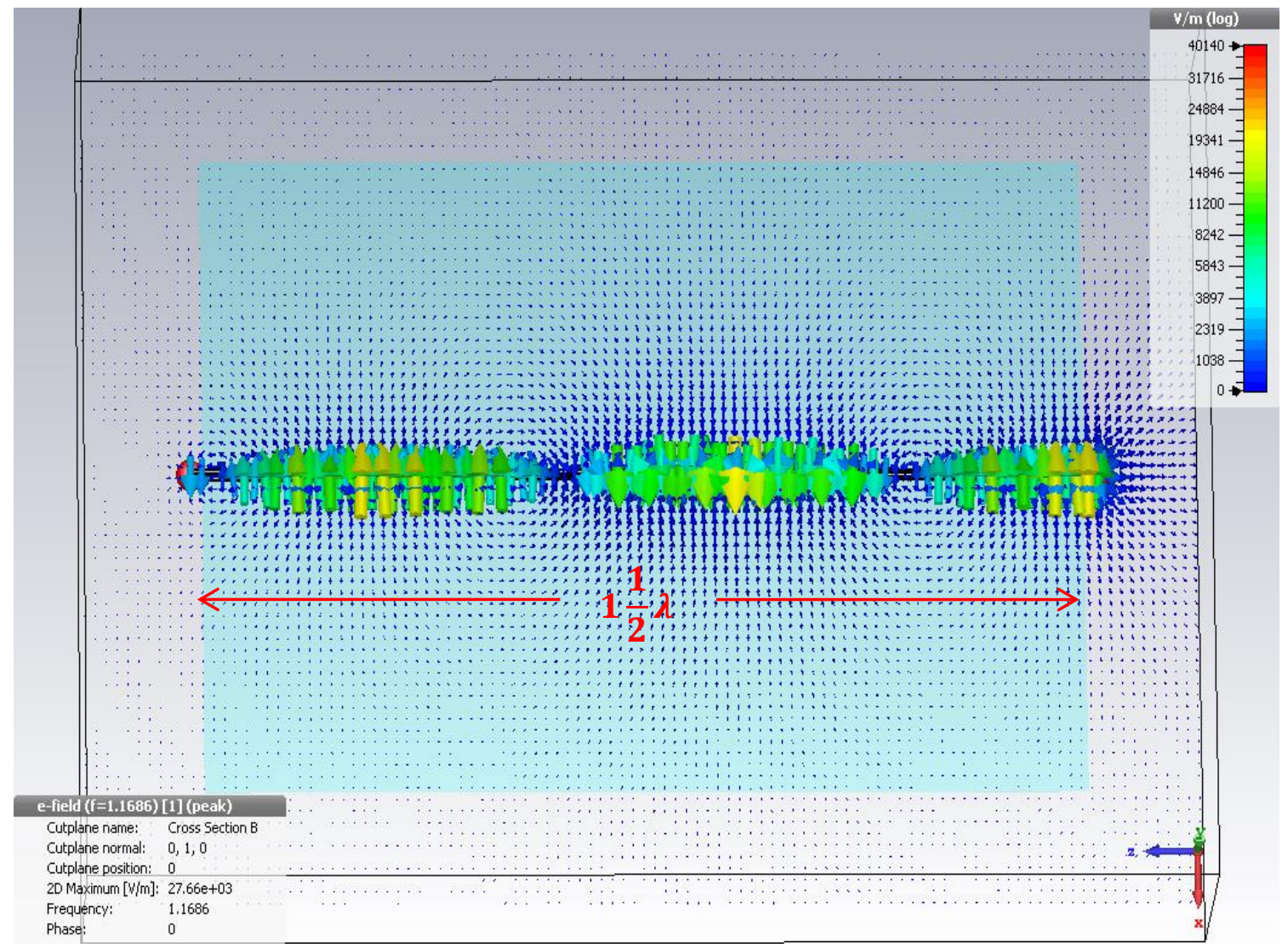

Figure 4.13 Top view of cross section of 3D fields on 2D plane of the stitched transmission line with stitch angle at $85^{\circ}$ used to compute its electrical length

b. Similarly, with the input impedance of the stitched transmission line with stitch angle at $65^{\circ}$ based on the peak $S_{11}$ at $1.034 \mathrm{GHz}$ is given as:

$$
\begin{gathered}
S_{11}=\frac{Z_{\text {in }}-50}{Z_{\text {in }}+50}=0.5272 \\
Z_{\text {in }}=177.0148 \Omega
\end{gathered}
$$


Similarly,

$$
\begin{gathered}
S_{21} \approx e^{-\propto l} \\
0.8199=e^{-\propto\left(150 \times 10^{-3}\right)}
\end{gathered}
$$

Thus,

$$
\begin{gathered}
\propto=1.3238 N p . m^{-1} \\
\alpha(-l)=\left(1.3238 \times\left(-150 \times 10^{-3}\right)+j 8.35\right) \\
\alpha(-l)=-0.1986 N p
\end{gathered}
$$

Here, $\beta l$ is determined from Fig.4.14, where $\beta l$ is given by:

$$
\beta l=7.94 \mathrm{rad}
$$

Thus the propagation constant is finally given as,

$$
\gamma(-l)=-0.29+j 7.94
$$

Equally, the difference in this values compared to the computed values can be attributed to the use of values from the s-parameter, unlike the computed values where line parameters were used.

Using,

$Z_{\text {in }}=Z_{0} \frac{Z_{L}+Z_{0} \tanh (\gamma(-l))}{Z_{0}+Z_{L} \tanh (\gamma(-l))}$

Solving for $Z_{0}$, using the quadratic formula gives:

$Z_{0}=\frac{\frac{-\left(z_{L}-Z_{\text {in }}\right)}{\tanh (\gamma(-l))} \pm \sqrt{\left(\frac{\left(Z_{L}-Z_{\text {in }}\right)}{\tanh (\gamma(-l))}\right)^{2}+4 Z_{\text {in }} Z_{L}}}{2}$

With $Z_{0}$ having two roots given by:

$$
Z_{01}=(79.41+j 4.06) \Omega
$$

Or 


$$
Z_{02}=\left(-1.01 \times 10^{2}+j 5.18\right) \Omega
$$

Here $Z_{01}=(79.41+j 4.06) \Omega$ is chosen as the characteristic impedance of the stitched transmission line. A choice of $Z_{02}=\left(-1.01 \times 10^{2}+j 5.18\right) \Omega$ translate to energy been generated within the transmission line that is proportional to the square of the impressed voltage or current. Thus this indicates that the transmission line contributes power to itself.

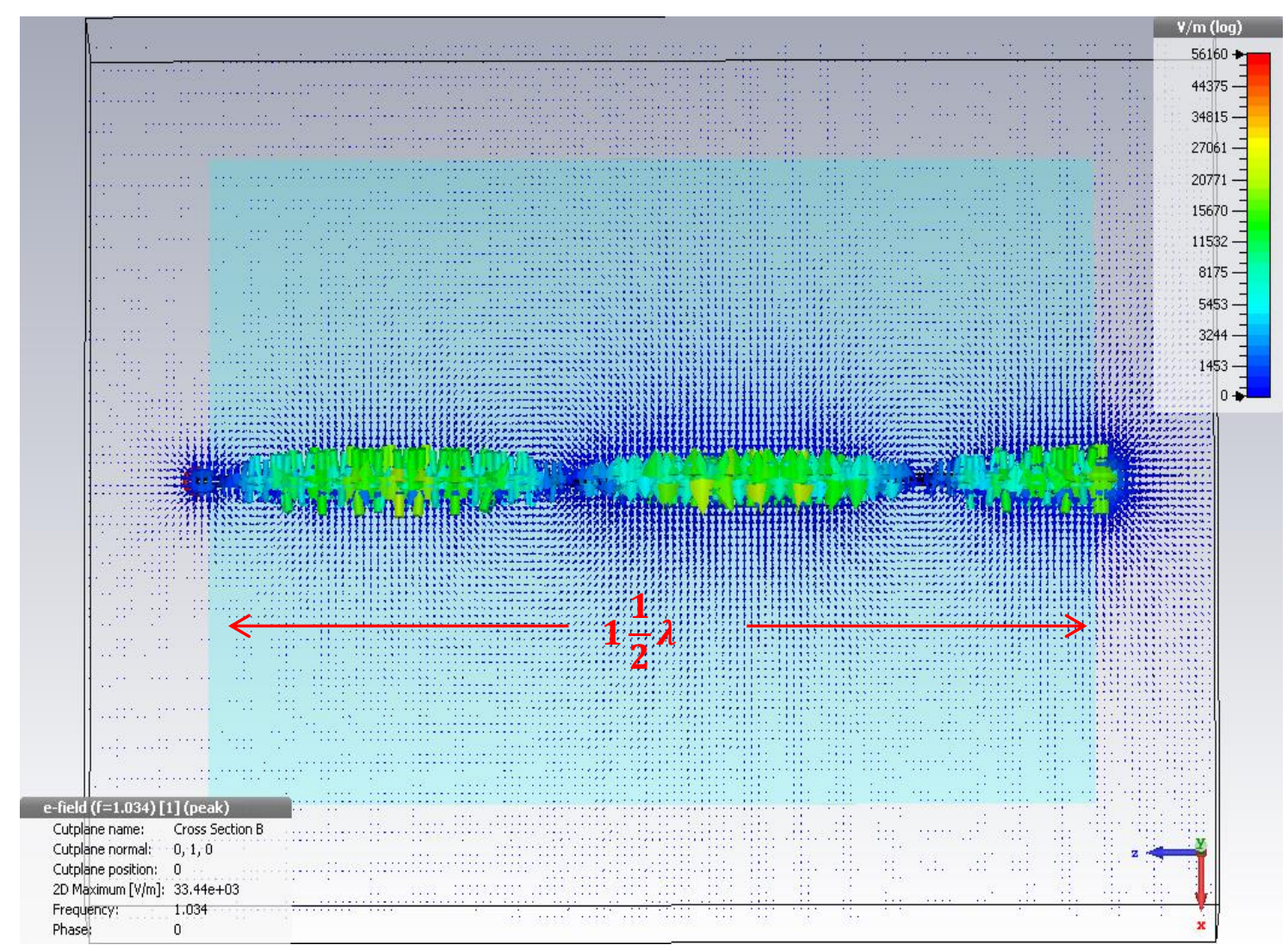

Figure 4.14 Top view of cross section of 3D fields on 2D plane of the stitched transmission line with stitch angle at $65^{\circ}$ used to compute its electrical length

c. Finally, with the input impedance of the stitched transmission line with stitch angle at $31^{\circ}$ based on the peak $S_{11}$ at $1.0973 \mathrm{GHz}$ is given as:

$$
\begin{gathered}
S_{11}=\frac{Z_{\text {in }}-50}{Z_{\text {in }}+50}=0.3293 \\
Z_{\text {in }}=99.0980 \Omega
\end{gathered}
$$

Here, 


$$
\begin{gathered}
S_{21} \approx e^{-\propto l} \\
0.9208=e^{-\propto\left(150 \times 10^{-3}\right)}
\end{gathered}
$$

Thus,

$$
\propto=0.5501 N p \cdot m^{-1}
$$

$$
\begin{gathered}
\alpha(-l)=\left(0.5501 \times\left(-150 \times 10^{-3}\right)+j 7.40\right) \\
\alpha(-l)=-0.0825 N p
\end{gathered}
$$

Here, $\beta l$ is determined from Fig. 4.15 , where $\beta l$ is given by:

$$
\beta l=7.40 \mathrm{rad}
$$

Thus the propagation constant is finally given as,

$$
\gamma(-l)=-0.08+j 7.40
$$

Likewise, the difference in this values compared to the computed values can be attributed to the use of values from the s-parameter, unlike the computed values where line parameters were used.

Using,

$$
Z_{\text {in }}=Z_{0} \frac{Z_{L}+Z_{0} \tanh (\gamma(-l))}{Z_{0}+Z_{L} \tanh (\gamma(-l))}
$$

Solving for $Z_{0}$, using the quadratic formula gives:

$$
Z_{0}=\frac{\frac{-\left(Z_{L}-Z_{\text {in }}\right)}{\tanh (\gamma(-l))} \pm \sqrt{\left(\frac{\left(Z_{L}-Z_{\text {in }}\right)}{\tanh (\gamma(-l))}\right)^{2}+4 Z_{\text {in }} Z_{L}}}{2}
$$

With $Z_{0}$ having two roots given by:

$$
Z_{01}=(65.68+j 1.90) \Omega
$$

Or 


$$
Z_{02}=(-75.37+j 2.17) \Omega
$$

Here $Z_{01}=(65.68+j 1.90) \Omega$ is chosen as the characteristic impedance of the stitched transmission line. A choice of $Z_{02}=(-75.37+j 2.17) \Omega$ translate to energy been generated within the transmission line that is proportional to the square of the impressed voltage or current. Thus this indicates that the transmission line contributes power to itself.

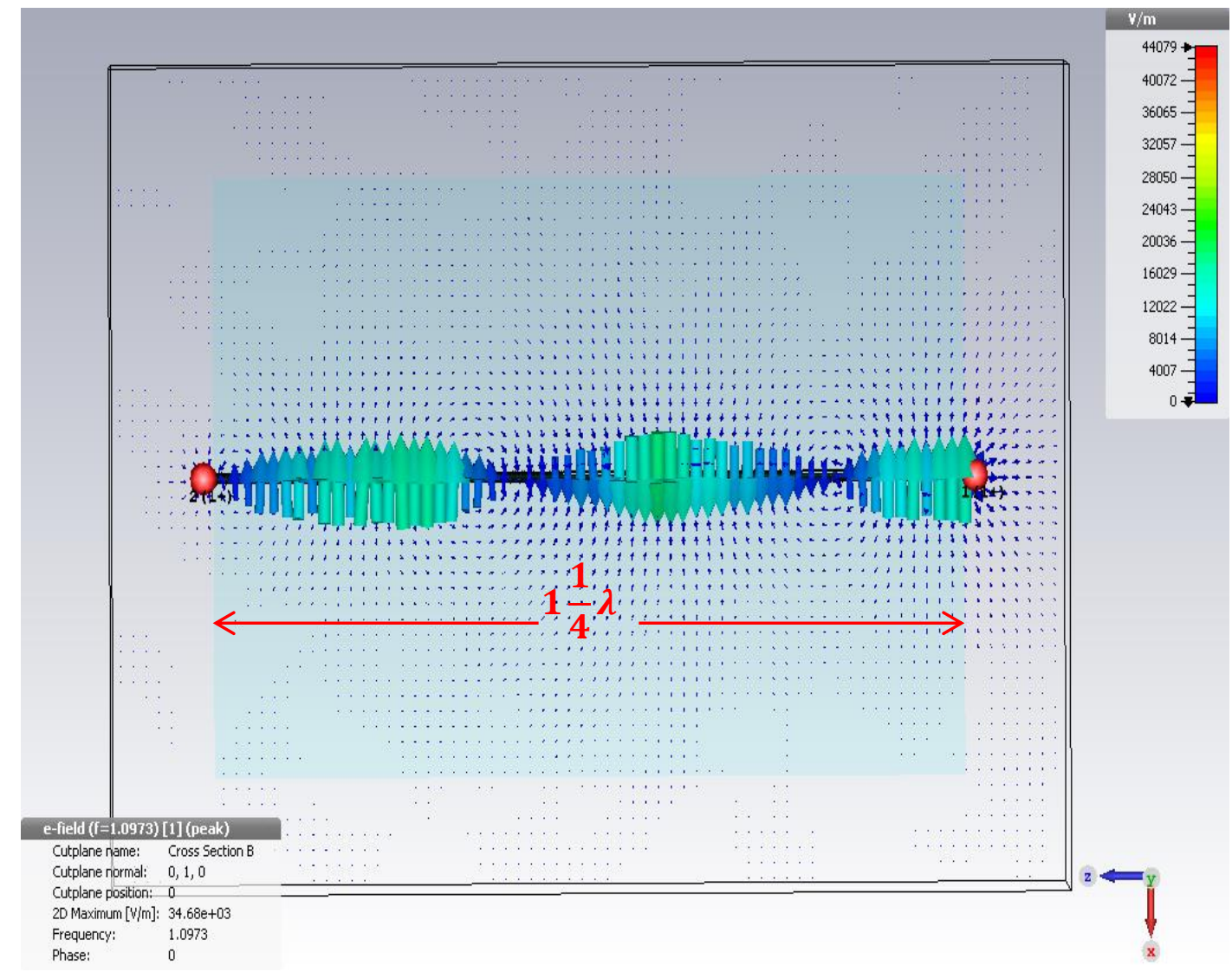

Figure 4.15 Top view of cross section of 3D fields on 2D plane of the stitched transmission line with stitch angle at $31^{\circ}$ used to compute its electrical length 
Return loss $\left(S_{11}\right)$ values from the scattering parameter plots with CST Microwave Studio Suite ${ }^{\circledR}$ at $1.1686 \mathrm{GHz}, 1.034 \mathrm{GHz}$ and $1.0973 \mathrm{GHz}$ for the three stitched angles $85^{\circ}, 65^{\circ}$ and $31^{\circ}$ were used to compute the input impedance, electrical length and characteristic impedance of the stitched transmission line. The characteristic impedances of the stitched transmission line at these frequencies are given as:

$$
\begin{aligned}
& Z_{01 \_85^{\circ}}=(71.61+j 29.93) \Omega \\
& Z_{02 \_65^{\circ}}=(79.41+j 4.06) \Omega \\
& Z_{\left.02 \_31\right)^{\circ}}=(65.68+j 1.90) \Omega
\end{aligned}
$$

From CST Microwave Studio Suite ${ }^{\circledR}$ simulation results, the characteristic impedance obtained from the 1D Results in the navigation tree, given as reference impedance $\left(Z_{R e f 1(1)}\right)$ are given as:

$$
\begin{aligned}
& Z_{\text {Ref1(1)(85) }}=45.521 \Omega \text {, } \\
& Z_{\operatorname{Ref1}(1)\left(65^{\circ}\right)}=45.431 \Omega \\
& Z_{R e f 1(1)(31)^{\circ}}=44.876 \Omega
\end{aligned}
$$

Compared with the calculated value of $Z_{0} \approx(45.569-i 0.028) \Omega$, it can be seen that the calculated and the $Z_{R e f 1(1)}$ values from CST Microwave Studio Suite ${ }^{\circledR}$ differ from the characteristic impedances that were computed from both the return loss $\left(S_{11}\right)$ and transmission coefficient $\left(S_{21}\right)$ results derived from CST Microwave Studio Suite ${ }^{\circ}$ from Fig.4.9, 4.12, 4.13 and 4.14. This is mainly because the stitched transmission lines were not matched at these frequencies. Usually, the characteristic impedance of a transmission line will vary with frequency and if the matching load fails to match the line at all frequencies, then the line will not be properly terminated and reflections will ensue [4.31]. Furthermore, increasing the RF frequency and the length of the cable also increases the insertion loss; as the higher the frequencies the greater the loss. In this case the length of the cables remains same. To minimise these effects, cables are made to have larger diameter as a cable with a lager diameter have less insertion loss and better power handling capabilities than a cable with a smaller diameter. 


\subsection{Conclusion}

In this chapter, a stitched wearable transmission line was designed and fabricated with three different stitch lengths corresponding to three different stitch angles. By using the idea presented by Wait [4.15] the stitched transmission line was designed with CST Microwave Studio Suite ${ }^{\circledR}$ and constructed with the aid of an improved presser foot using conductive threads from Light Stitches®. The simulated results from CST Microwave Studio Suite indicated that DC losses tend to increase with an increase in stitch angle which results in an increase in a number of stitches. The losses were also seen to increase with an increase in frequency. Losses attributed to the stitch transmission line include Dielectric loss, mismatch loss, radiation and induction loss. The radiation loss generally is minimised with increase in number of stitches or decrease in stitch angle as more shield coverage means less radiation of energy; however, this does not necessarily mean less signal attenuation as stated earlier, as it can be seen that the transmission line with the least stitch angle $31^{\circ}$ has more losses compared to ones with $65^{\circ}$ and $85^{\circ}$.

A comparison of the computed characteristic impedance, characteristic impedance from CST referred to as $Z_{R e f 1(1)}$, with the characteristic impedance from the scattering parameters also from CST was carried out and results presented.

Measurements carried out on the stitched transmission line alongside side the DC analysis of the shield and computation of its transfer impedance at low frequencies $(<1 \mathrm{GHz})$ are presented in chapter five. 


\section{REFERENCE}

4.1. D. Cottet, J. Grzyb, T. Kirstein and G. Tröster, "Electrical Characterisation of Textile Transmission Lines," IEEE Transaction on Advance Packaging, Vol. 26, N0. 2, pp. 182-190, May 2003

4.2. T. Kirstein, D. Cottet, J. Grzyb and G. Tröster, "Textiles for Signal Transmission in Wearables," Proceedings Workshop on Modelling, Analysis and Middleware Support for Electronic Textiles MAMSET, San Jose, CA, 6, pp. 9-14, October 2002

4.3. T. Acti, S. Zhang, A. Chauraya, W. Whittow, R. Seager, T. Dias and Y. Vardaxoglou, "High-Performance Flexible Fabric Electronics for Megahertz Frequency Communications", Loughborough Antennas \& Propagation Conference, pp. 1-4, November 2011

4.4. M. Choi and J. Kim, "Electrical Characteristics and Signal Transmission Characteristics of Hybrid Structure Yarns for Smart Wearable Devices," Fibers and Polymers, Vol.17, No.12, pp. 2055-2061, 2016

4.5. I. Locher and G. Tröster, "Screen-Printed Textile Transmission Lines," Textile Research Journal, Vol. 77(11), pp. 837-842, December 2007

4.6. Z. Xu, T. Kaufmann, C. Fumeaux, "Wearable Textile Shielded Stripline for Broadband Operation," Microwave and Wireless Components Letters, IEEE, vol.24, no.8, pp.566-568, August 2014

4.7. J. Leśnikowski, "Textile Transmission Lines in the Modern Textronic Clothes," 89 Fibres \& Textiles in Eastern Europe, Vol. 19, No. 6 (89) pp. 89-93, 2011

4.8. I. Locher, T. Kirstein, G. Troster. "Routing Methods Adapted to e-Textiles," ad. of Proc. 37th Int. Symp. Microelectron (IMAPS 2004), November 2004

4.9. C. R. Merritt, B. Karaguzel, T. Kang, J. M. Wilson, P. D. Franzon, H.T. Nagle, B. Pourdeyhimi and E. Grant "Electrical Characterization of Transmission Lines on Specific Nonwoven Textile Substrates,” MRS Proceedings, 870, H4.7 doi:10.1557/PROC-870-H4.7, 2005

4.10. M. Chedid, P. Leisner and I. Belov, "Experimental analysis and modeling of textile transmission line for wearable applications," International Journal of Clothing Science and Technology, Vol. 19 No. 1, pp. 59-71, 2007

4.11. J. Jeon, S. Kim, J. Koo, S. Hong, Y. Moon, S. Jung and B. Kim, "Electrical Characterisation of Differential Stretchable Transmission Line," Microwave 
Symposium Digest (MTT), 2011 IEEE MTT-S International, vol., no., pp.1,1, 5-10 June 2011

4.12. J. Leśnikowski, "Textile Transmission Lines in the Modern Textronic Clothes," FIBRES \& TEXTILES in Eastern Europe, Vol. 19, No. 6 (89) pp. 89-93, 2011

4.13. Presser Foot [Online]. [Accessed: 12-February 2017]. Available from: https://en.wikipedia.org/wiki/Presser_foot

4.14. E. Vance, "Shielding effectiveness of braided-wire shields," IEEE Transactions on Electromagnetic Compatibility, vol. EMC-17, pp. 71-77, May 1975

4.15. J. R. Wait, "Electromagnetic Theory of the Loosely-Braided Coaxial Cable: Part I," in IEEE Transactions on Microwave Theory and Techniques, vol. 24, no. 9, pp. 547553, September 1976

4.16. CST Studio Suite ${ }^{\circledR}$ [Online]. [Accessed: 12-February 2017]. Available from: https://www.cst.com/Products/CSTS2

4.17. M. A. R. Gunston, Microwave Transmission Line Impedance Data. New York: Van Nostrand Reinhold, 1972.

4.18. H. J. Riblet, "An accurate determination of the characteristic impedance of the coaxial system consisting of a square concentric with a circle," IEEE Trans. Microwave Theory Tech., vol. MTT-23, pp. 714-715, Aug. 1975.

4.19. H. J. Riblet, "An accurate approximation of the impedance of a circular cylinder concentric with an external square tube," IEEE Trans. Microwave Theory Tech., vol. MTT-31, pp. 841-844, Oct. 1983.

4.20. P. A. A. Laura and L. E. Luisoni, "Approximate determination of the characteristic impedance of the coaxial system concentric with a circle," IEEE Trans. Microwave Theory Tech., vol. MTT-25, pp. 160-161, Feb. 1977.

4.21. T. K. Sheshadri and K. Rajaian, "Accurate estimation of characteristic impedance of coaxial transmission line problems by the eigenfunction approach," Proc. IEEE, vol. 70, pp. 82-83, J . 1982.

4.22. W. Lin, "A critical study of the coaxial transmission line utilizing conductors of both circular and square cross-section; IEEE Trans. Microwave Theory Tech., pp. 19811988, Nov. 1982.

4.23. W. Lin, "Polygonal coaxial line with round center conductor," IEEE Trans. Microwave Theory Tech., vol. MTT-33, pp. 545-550, June 1985. 
4.24. H. Estevez, E. Moreno and F. Ares, "A method of Approximating the characteristic Impedance of Coaxial Lines in Which the Inner Conductor is circular and the Outer is Polygonal," IEEE Trans. On Microwave Theory and Techniques, vol. 37, no.3, March 1989.

4.25. S. G. Pan, "Approximate Determination of the Characteristic impedance of the Coaxial System Consisting of an Irregular Outer Conductor and a Circular Inner Conductor," IEEE Trans. On Microwave Theory and Techniques, vol. MTT-35, no.1, January 1987.

4.26. S. G. Pan, "Characteristic Impedance of a Coaxial System Consisting of Circular and Noncircular Conductors," IEEE Trans. On Microwave Theory and Techniques, vol. 36, no.5, May 1988.

4.27. R. Ludwig and G. Bogdanov, "RF Circuit Design: Theory and Applications" 2nd edition, Prentice-Hall, 2000.

4.28. M. N. O. Sadiku, "Elements of Electromagnetics," Sixth Edition, Oxford University Press, 2014.

4.29. A. R. Djordjevic, A. G. Zajic, D. V. Tosic and T. Hoang, "A Note on the Modelling of Transmission-Line Losses," IEEE Transactions on Microwave Theory and Techniques, vol. 51, no. 2, pp.483-486, February 2003

4.30. D. M. Pozar, "Microwave Engineering," Fourth Edition, John Wiley \& Sons, Inc., 2012

4.31. E. Dahlman, C. Oestges, A. C. Bovik, B. A. Fette, K. Jack, F. Dowla, S. Parkvall, J. Skold, C. DeCusatis, E. Silva, R. Olexa, B. Clerckx, L. M. Correia, P. A Chou, M. Van der Schaar, W. K. Ling, R. Kitchen, D. M. Dobkin, D. Bensky, D. Morgan, J. Ellis, C. Pursell, J. Rahman, L. Guibas, F. Zhao, "Communications Engineering and Mega-Reference,” Academic Press, pp. 54, 2009 


\section{Chapter 5}

\section{Experimental Measurements on the Stitched Transmission Line}

This chapter presents RF measurements that were carried out with the stitched transmission line using an Anritsu MS46524A 7GHz Vector Network Analyser (VNA) for a frequency range of $0.04-4 G \mathrm{~Hz}$, the DC analysis and the computation of the transfer impedance of the stitched shield for frequencies $<1 \mathrm{GHz}$. Measurements were carried out on the stitched transmission line constructed first with conductive threads and copper wires and secondly with conductive threads only.

\subsection{Introduction to RF Measurement on the stitched Transmission Line}

Measurements are the final and the most important step to characterize the stitched wearable transmission line system as all the practical applications rely on measurements results. Measurements setups require a good knowledge of theory, patience and time. Hence once the stitched transmission line is designed and fabricated, the next step is to carry out measurements on it to see how the transmission line measured results agree with both theoretical and simulated results.

Since the wearable transmission line will be operating under the movement of the human body some specific deformations on the transmission line such as bending and crumpling should be examined during measurement. Similarly, the stitched transmission line should be measured under different environmental conditions such as mist, snow, rain, dew, high humidity, dust, mud etc. Also because the wearable transmission line is supposed to be part of the apparel, testing the durability of the transmission line in terms of performance after washing is also a requirement. The kind of wash, which could be either hand or machine wash, should also be taken into considerations. The first is considered with regards to developing countries where washing of apparels is normally done locally with the hands or when caught up in war situations where the only option is to use the hand to wash the apparel, while the type of wash considered in the latter is based on the different functions on the washing machine and temperatures and duration of the process. Furthermore, the 
repeatability otherwise known as precision is very important in evaluating the performance of the stitched transmission lines. To test that, five repeated measurements were made on the stitched transmission line with the zig-zag stitch having stitch angles of $85^{\circ}, 65^{\circ}$ and $31^{\circ}$.

The stitched transmission lines made with conductive threads and copper wires, with three different stitch angles and stitch patterns, two different bending angle and substrates and after subjecting it to washing cycles were tested. Finally, on-body measurements were also carried on the stitched transmission line. The scattering parameter measurements were carried out with an Anritsu MS46524A 7GHz Vector Network Analyser. The measurement set up is as shown in Fig 5.1.

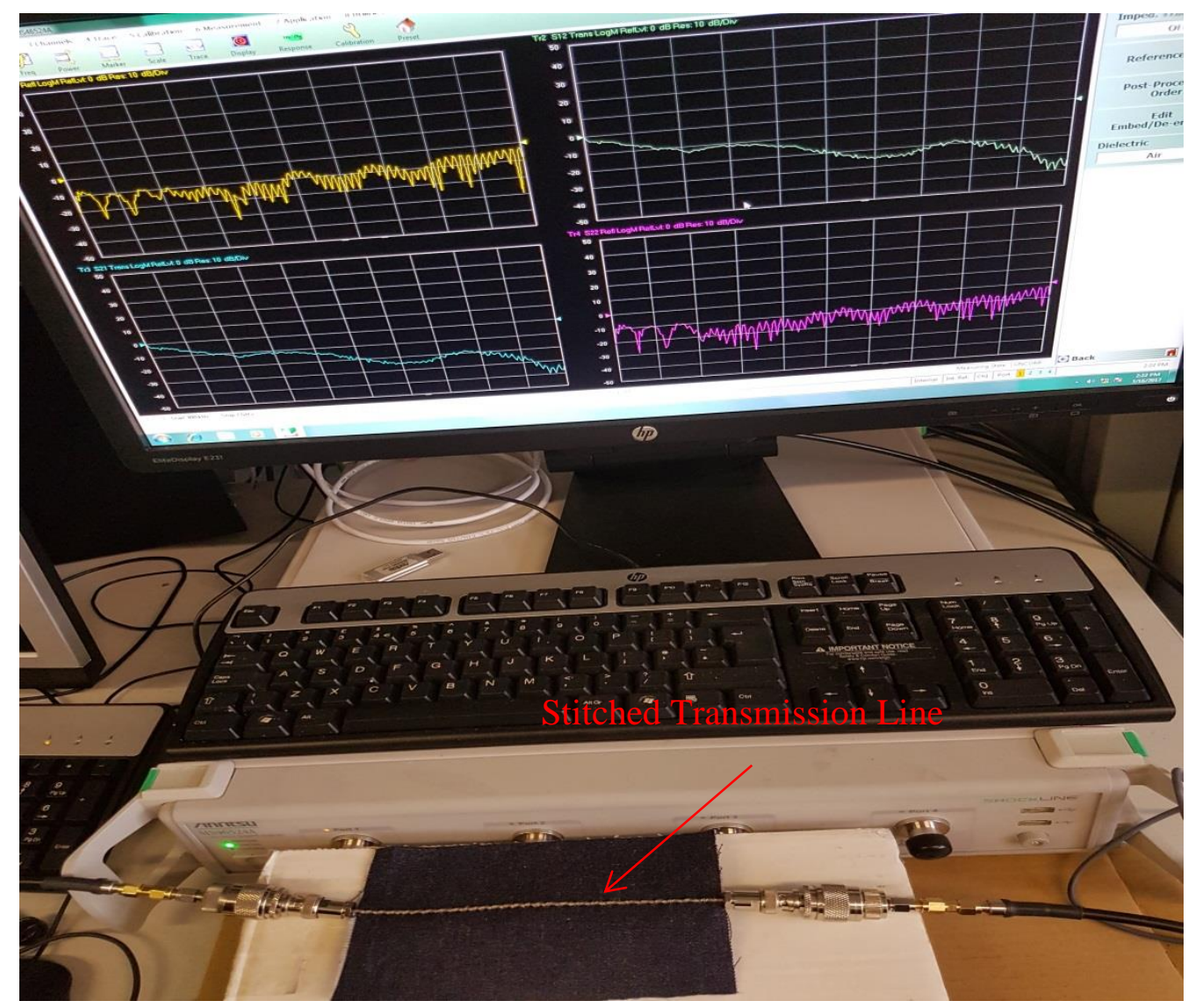

Figure 5.1 Measurement setup with Anritsu MS46524A 7GHz Vector Network Analyser 


\subsection{Stitched Transmission Line with Conductive Thread and Copper Wire}

Measurements were carried out on the stitched transmission line fabricated with copper wire and conductive thread from Light Stitches within the frequency range of $0.04-4 \mathrm{GHz}$ with the scattering parameter results as indicated in Fig.5.2, 5.4 and 5.6.

Since the resistance of the conductive thread is not zero, whenever current flows through the conductive threads that make up the shield, some energy is lost in the form of heat. This loss increases with the square of the current and referred to as resistive loss. Resistive losses on the stitched transmission line were observed for frequencies up to $1 \mathrm{GHz}$ with the losses increasing with increased stitched length.

Suppose a signal traveling within the stitched transmission line encounters a discontinuity in the characteristic impedance of the stitched transmission line or if the end of the stitched transmission line is not properly terminated in its characteristic impedance the signal is reflected back in the opposite direction in what is termed as mismatch loss. Since the impedance of the stitched transmission line is not perfectly matched, we expect some losses due to reflections, which are as a result of mismatching of the source and load even when the cable has no internal loss. From Fig. 5.2, 5.4 and 5.6, the reflection coefficient, $S_{11}$, indicates how much power is reflected back to port 1 compared to the power supplied to it. Similarly, when power is supplied by a source and it is not reflected back or available at the load, radiation losses are said to occur. Absorption lossess casued by the dissipation or conversion of electrical or electromagnetic energy into other foms of energy mainly heat also take place. From Fig. 5.2, 5.4 and 5.6, the transmission coefficient, $S_{21}$, represents the power received at port 2 due to power input at port port 1.

Here, $C W_{-} C T_{-} L 2, C W_{-} C T_{-} L 3$ and $C W_{-} C T_{-} L 4$ refers to the stitched transmission lines constructed with copper wire and conductive thread, with stitch length at $2 \mathrm{~mm}, 3 \mathrm{~mm}$ and $4 \mathrm{~mm}$ respectively. Also, $C T_{-} L 2, C T_{-} L 3$ and $C T_{-} L 4$ refers to the stitched transmission line constructed with conductive thread only, with stitch length at $3 \mathrm{~mm}$, while Sim_L2, Sim_L3 and Sim_L4 refers to the simulated results with annealed copper wires with stitch length at $2 \mathrm{~mm}, 3 \mathrm{~mm}$ and $4 \mathrm{~mm}$ respectively.

From Fig. 5.2, 5.4 and 5.6, measured reflection coefficient $S_{11}$ are below $-5.19 d B$ for frequencies up to $3.161 \mathrm{GHz}$ for $S I M_{-} L 2$ and $-5 \mathrm{~dB}$ and $-6.66 \mathrm{~dB}$ for $C W_{-} C T_{-} L 2$ and $C T_{-} L 2$ 
in all of the operation band respectively, while the transmission coefficients $S_{21}$ are better than $-2.14 d B$ for frequencies up to $2.478 \mathrm{GHz}$ for $S I M_{-} L 2$ and $-7.6 \mathrm{~dB}$ for frequencies up to $0.9673 \mathrm{GHz}$ and $-6 \mathrm{~dB}$ for frequencies up to $1 \mathrm{GHz}$, for $C W_{-} C T_{-} L 2$ and $C T_{-} L 2$ respectively. Equally, the measured reflection coefficient $S_{11}$ are below $-2.79 \mathrm{~dB}$ in all of the operation band for $S I M_{-} L 3$ and $-4.96 \mathrm{~dB}$ and $-5.88 \mathrm{~dB}$ for $C W_{-} C T_{-} L 3$ and $C T_{-} L 3$ in all of the operation band respectively. The transmission coefficients $S_{21}$ are better than $-4 d B$ for frequencies up to 3.408GHz for $S I M \_L 3$ and $-6.36 \mathrm{~dB}$ for frequencies up to $1.1 \mathrm{GHz}$ and $-6 \mathrm{~dB}$ for frequencies up to $1.329 \mathrm{GHz}$, for $C W_{-} C T_{-} L 3$ and $C T_{-} L 3$ respectively. Finally, measured reflection coefficient $S_{11}$ are below $-3.2 \mathrm{~dB}$ for frequencies up to $3.161 \mathrm{GHz}$ for $S I M_{-} L 4$ and $-4.6 d B$ and $-5.53 d B$ for $C W_{-} C T_{-} L 4$ and $C T_{-} L 4$, and the transmission coefficients $S_{21}$ are better than $-3.3 \mathrm{~dB}$ for frequencies up to $3.08 \mathrm{GHz}$ for $S I M_{-} L 4$ and $-6.5 \mathrm{~dB}$ for frequencies up to $1.195 \mathrm{GHz}$ and $-5.6 \mathrm{~dB}$ for frequencies up to $2.04 \mathrm{GHz}$, for $C W_{-} C T_{-} L 4$ and $C T_{-} L 4$ respectively.

The stitched transmission line $C W_{-} C T_{-} L 2$ is seen to be more lossy compared to $C T_{-} L 2$ for frequencies up to $0.9 \mathrm{GHz}$. Equally, the stitched transmission line $C W_{-} C T_{-} L 3$ is seen to be more lossy compared $C T_{-} L 3$ for frequencies up to $1.6 \mathrm{GHz}$. Finally, the stitched transmission line $C W_{-} C T_{-} L 4$ is also seen to be more lossy compared $C T_{-} L 4$ for frequencies up to $2.6 \mathrm{GHz}$. The losses associated with the stitched transmission line like every other transmission line are the real loss and mismatch. The real loss is the conductor loss, dielectric loss and radiation or induction loss, while the mismatch loss is as a result of reflections along the transmission line as it encounters a discountinuity in the characteristic impedance of the line or if the stitched transmission line is not terminated at its characteristic impedance. For the stitched transmission line, the dielectric loss is same for the three, but that cannot be said with the conductor loss, radiation loss or induction loss and mismatch loss. The radiation loss however, is more dominant in this case because of the sparsed nature of the shield of the stitched transmission line and this loss tends to increase with increase in frequency. The possiblity of also having more of the induction loss along with the dominant radiation loss in the stitched transmission line based on its design features cannot be ruled out. The induction loss occurs when the electromagnetic fields cuts across any conductor and current is been induced in the conductor. When this occurs power is dissipated in the conductor and this is then lost as heat. Additional losses can also be ascribed to the loose connection between the copper wire and conductive thread which could lead to an increase in resistance at the connection. 

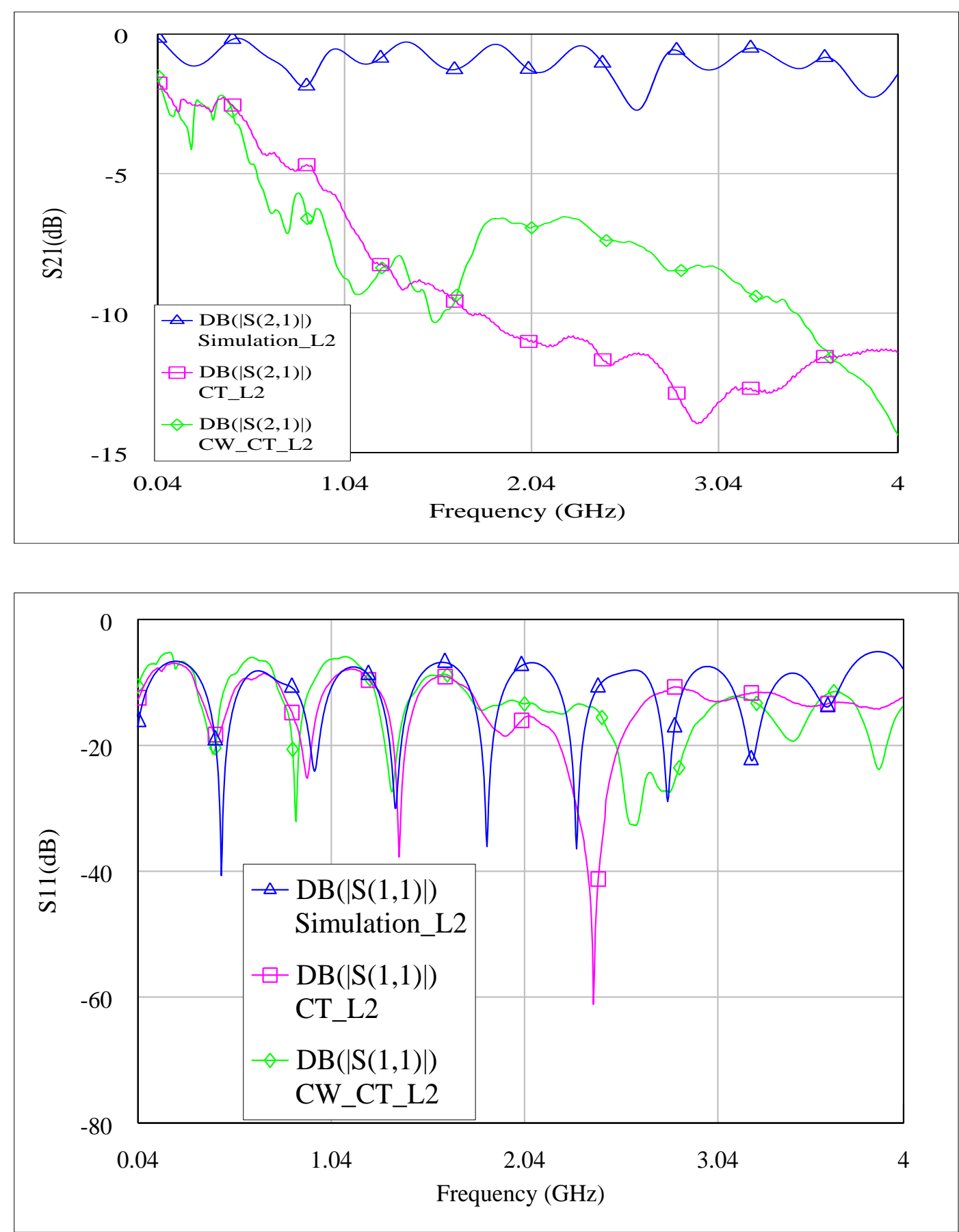

Figure 5.2 Measured S-Parameters of stitched transmission line with $C W_{-} C T_{-} L 2, C T_{-} L 2$ and $S I M \_L 2$ 


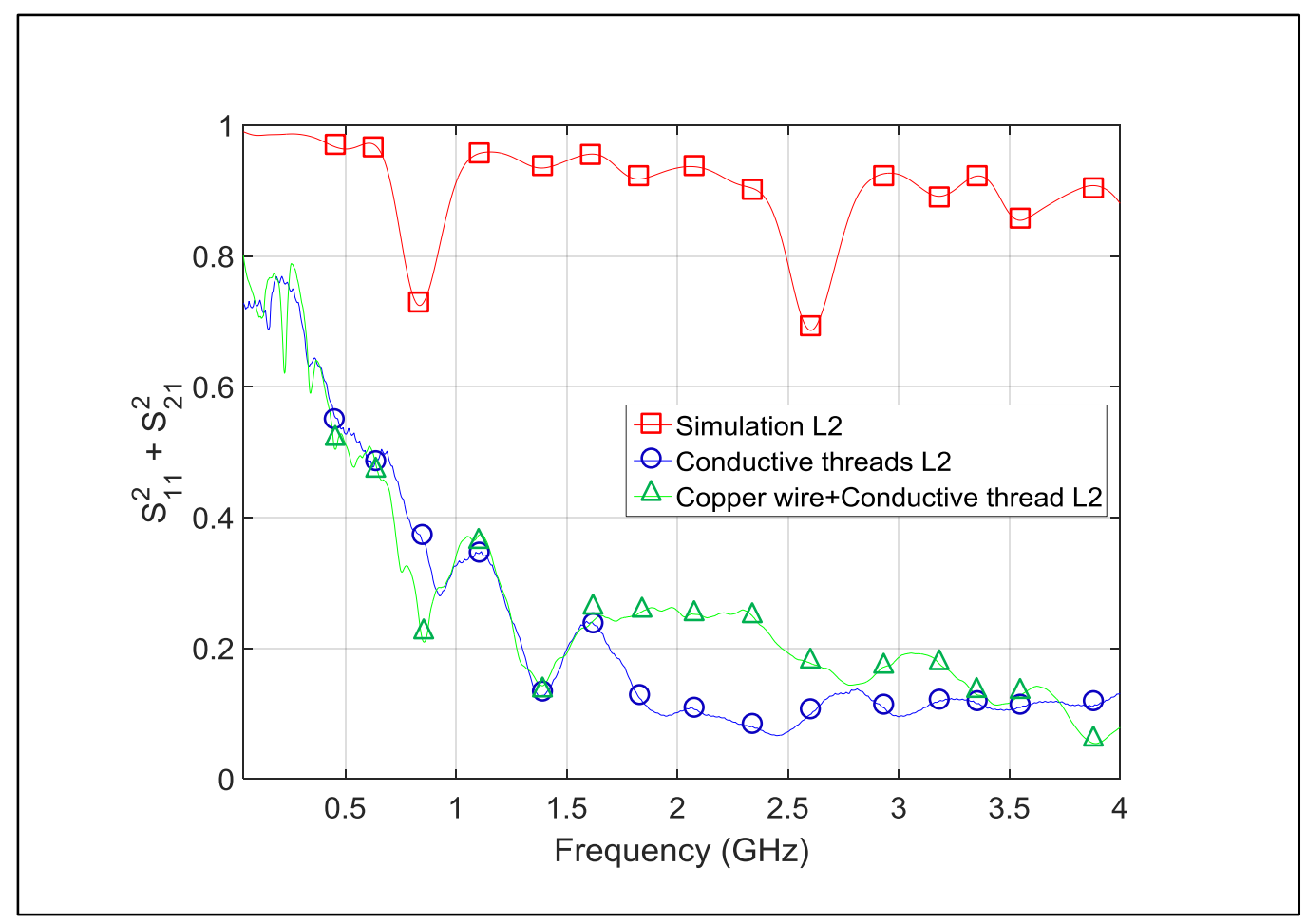

Figure 5.3 Plot of $\boldsymbol{S}_{\mathbf{1 1}}^{\mathbf{2}}+\boldsymbol{S}_{\mathbf{2 1}}^{\mathbf{2}}$ against frequency of stitched transmission line with CW_CT_L2, CT_L2 and SIM_L2 

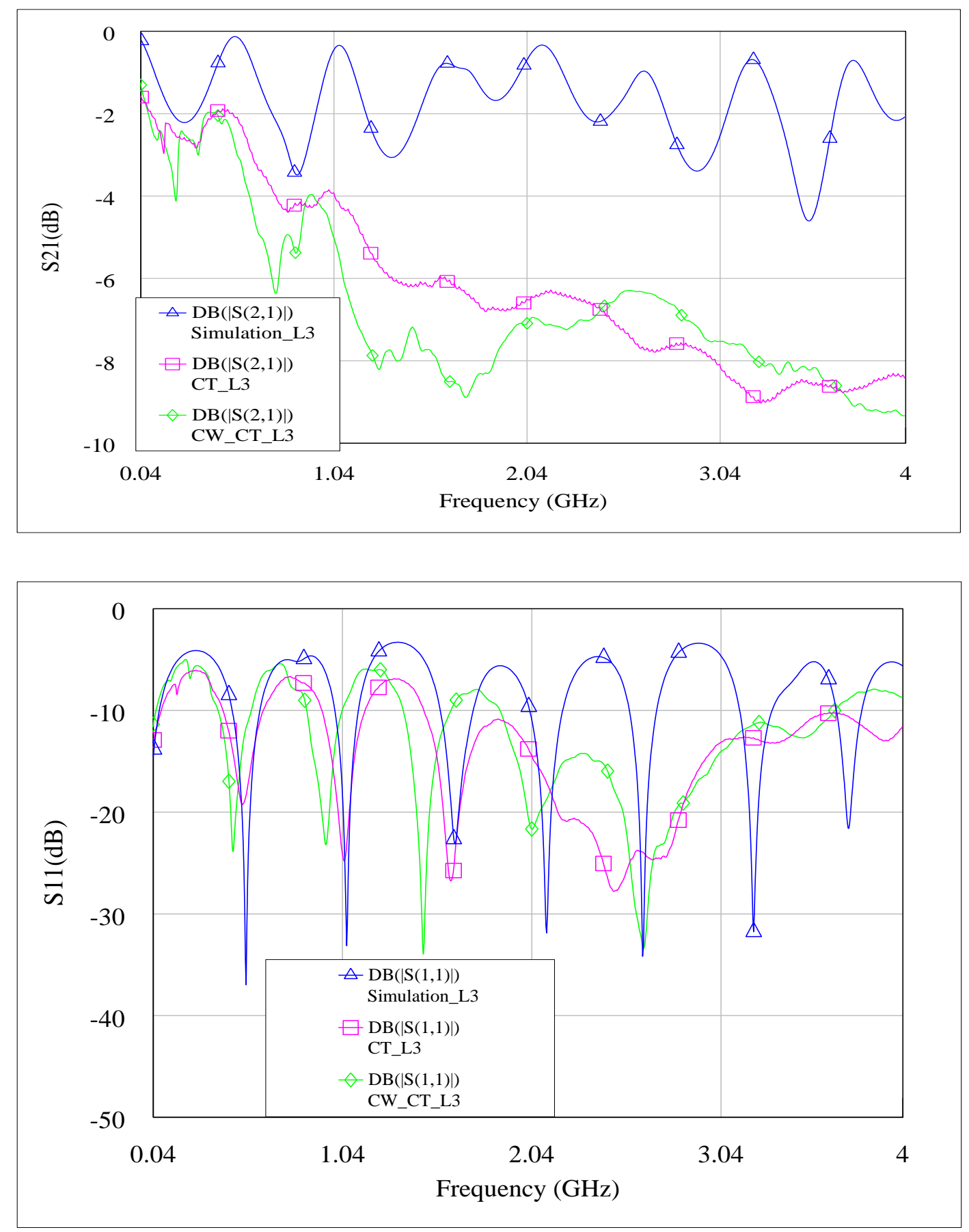

Figure 5.4 Measured S-Parameters of stitched transmission line with $C W_{-} C T_{-} L 3, C T_{-} L 3$ and SIM_L3 


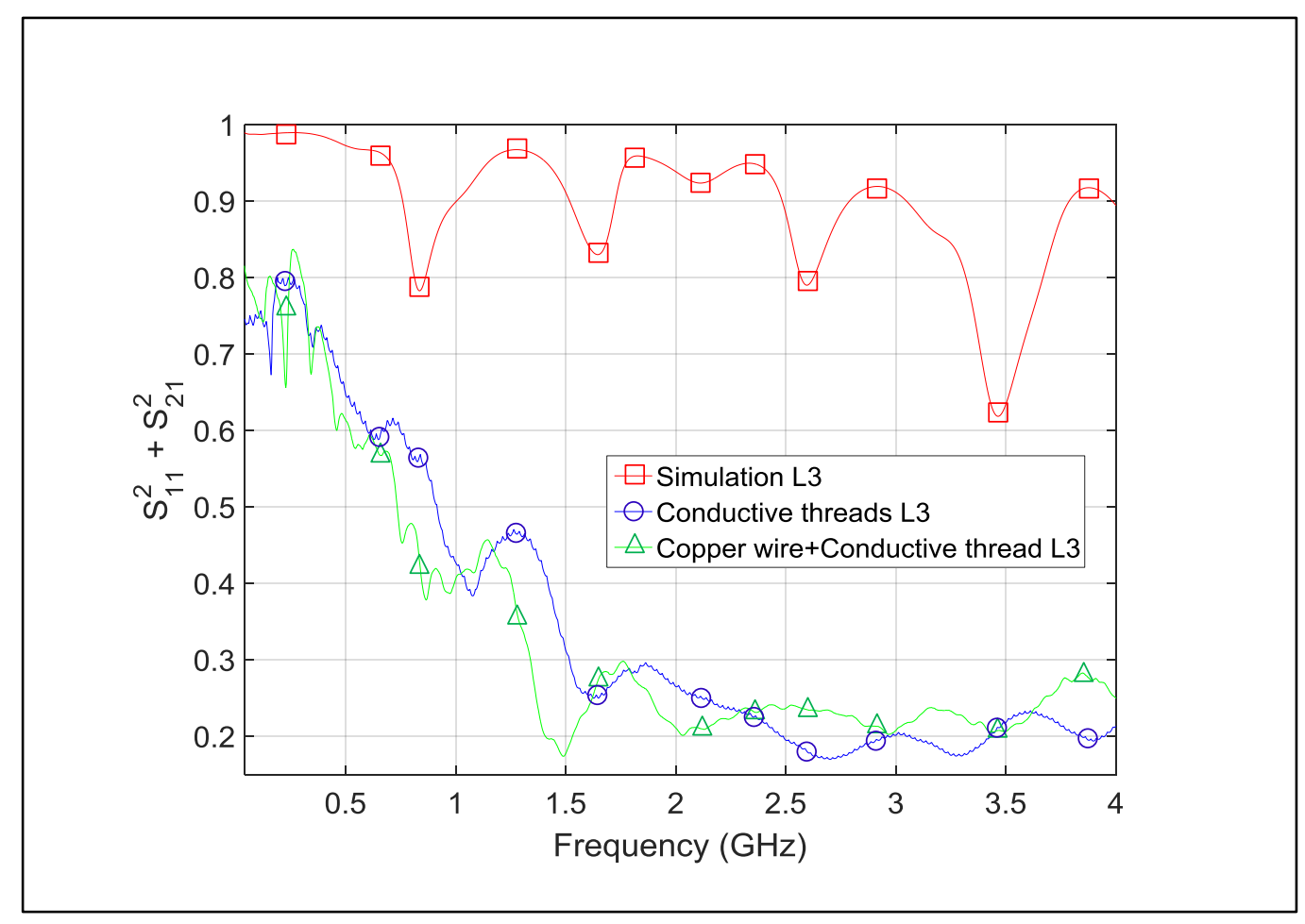

Figure 5.5 Plot of $\boldsymbol{S}_{\mathbf{1 1}}^{\mathbf{2}}+\boldsymbol{S}_{\mathbf{2 1}}^{\mathbf{2}}$ against frequency of stitched transmission line with CW_CT_L3, CT_L3 and SIM_L3 

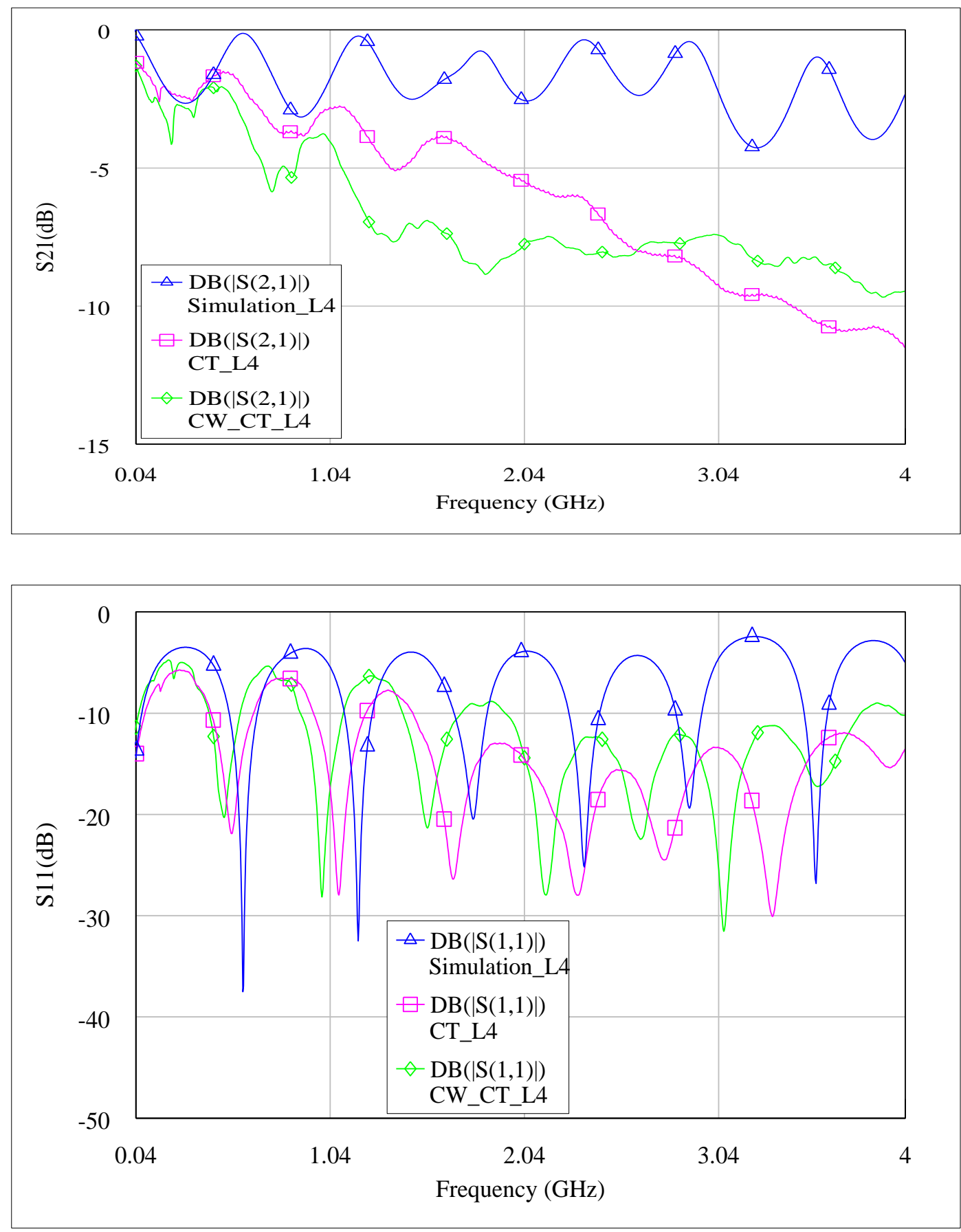

Figure 5.6 Measured S-Parameters of stitched transmission line with CW_CT_L4, CT_L4 and SIM_L4 


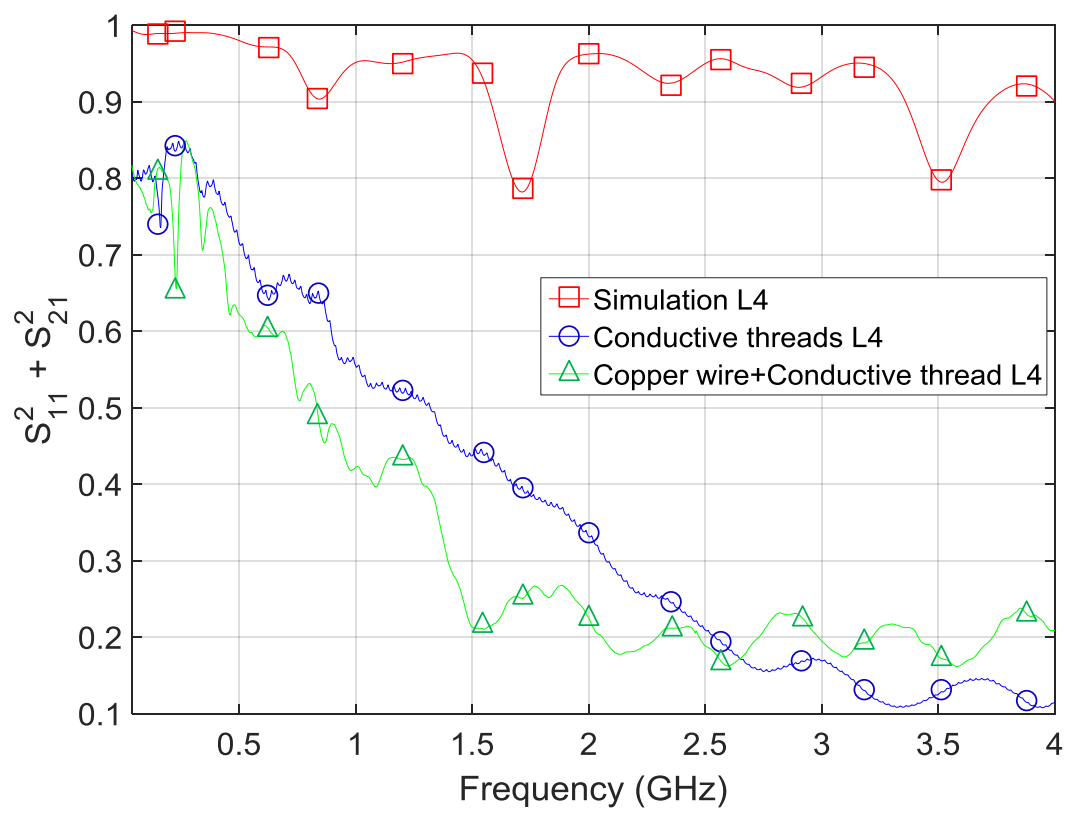

Figure 5.7 Plot of $\boldsymbol{S}_{\mathbf{1 1}}^{\mathbf{2}}+\boldsymbol{S}_{\mathbf{2 1}}^{\mathbf{2}}$ against frequency of stitched transmission line with CW_CT_L4, CT_L4 and SIM_L4

\subsection{Stitched Transmission Line with Conductive Threads}

Measurements on the stitched transmission line within a frequency range of $0.04-4 \mathrm{GHz}$ were also carried out using conductive threads only from Light Stitches with different stitch angles, stitch types, different curved bending angles and when subjected to washing cycles as well as on-body measurements. These are discussed in the subsequent sections.

\subsubsection{Stitched Transmission Line with Three Different Stitch Angles}

With the zig-zag stitch set on the sewing machine, there different stitched transmission lines were fabricated with the three different stitch angles as depicted in Fig.5.8. The measured scattering parameters from 0.04 to $4 \mathrm{GHz}$ are presented in Fig.5.9, 5.10 \& 5.11.

The reflection coefficient $S_{11}$ for stitch angle $85^{\circ}$ are below $-10 \mathrm{~dB}$ in most of the operation band, while the transmission coefficients $S_{21}$ are better than $-8 d B$ for frequencies up to $2.5 \mathrm{GHz}$. Equally the measured reflection coefficient $S_{11}$ for stitch angle $65^{\circ}$ are below $-10 \mathrm{~dB}$ 
in most of the operation band, while the transmission coefficients $S_{21}$ are better than $-10 \mathrm{~dB}$ for frequencies up to $2.16 \mathrm{GHz}$. Finally, measured reflection coefficient $S_{11}$ for stitch angle $31^{\circ}$ are below $-10 \mathrm{~dB}$ for frequencies up to $2.55 \mathrm{GHz}$ in most of the operation band, and the transmission coefficients $S_{21}$ are better than $-15 \mathrm{~dB}$ for frequencies up to $2.5 \mathrm{GHz}$.

The apertures were seen to be bigger with the stitched transmission line having a stitch angle of $85^{\circ}$ and 60 stitches, as this has less shield coverage compared to the stitched transmission line with stitch angles of $65^{\circ}$ and $31^{\circ}$ which has 90 and 162 stitches respectively. It is expected that the less the shield coverage the more the radiation loss and vice versa, with the radiation loss increasing with increase in frequency. The DC losses were more with stitch angles of $65^{\circ}$ and $31^{\circ}$ because more threads were used in the construction of the stitched transmission lines compared to the one with a stitch angle of $85^{\circ}$. Some ripples were also observed at higher frequencies, which are mainly as a result of multiple reflections along the transmission line. 


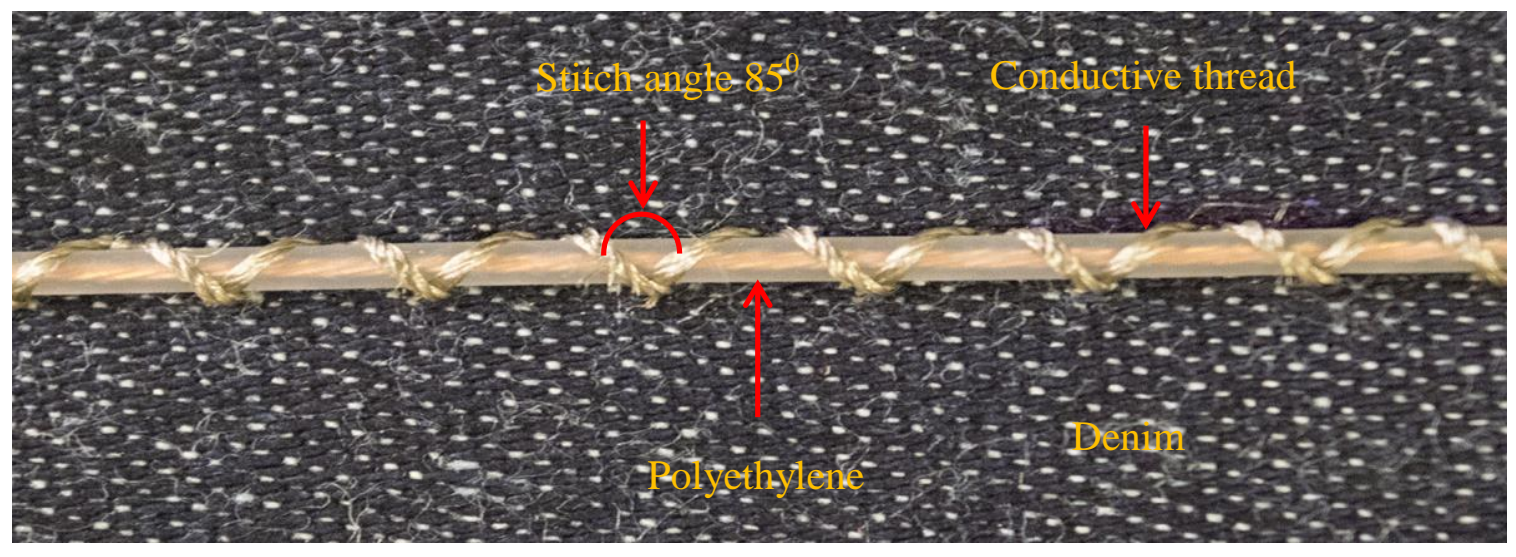

(a)

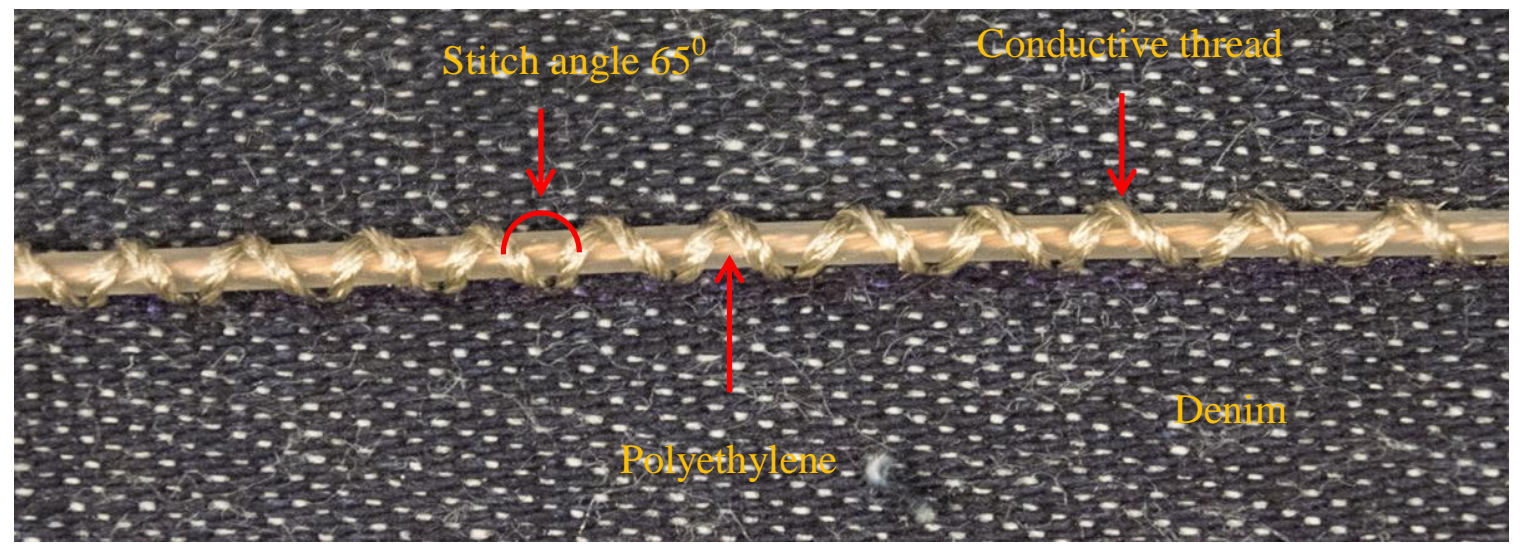

(b)

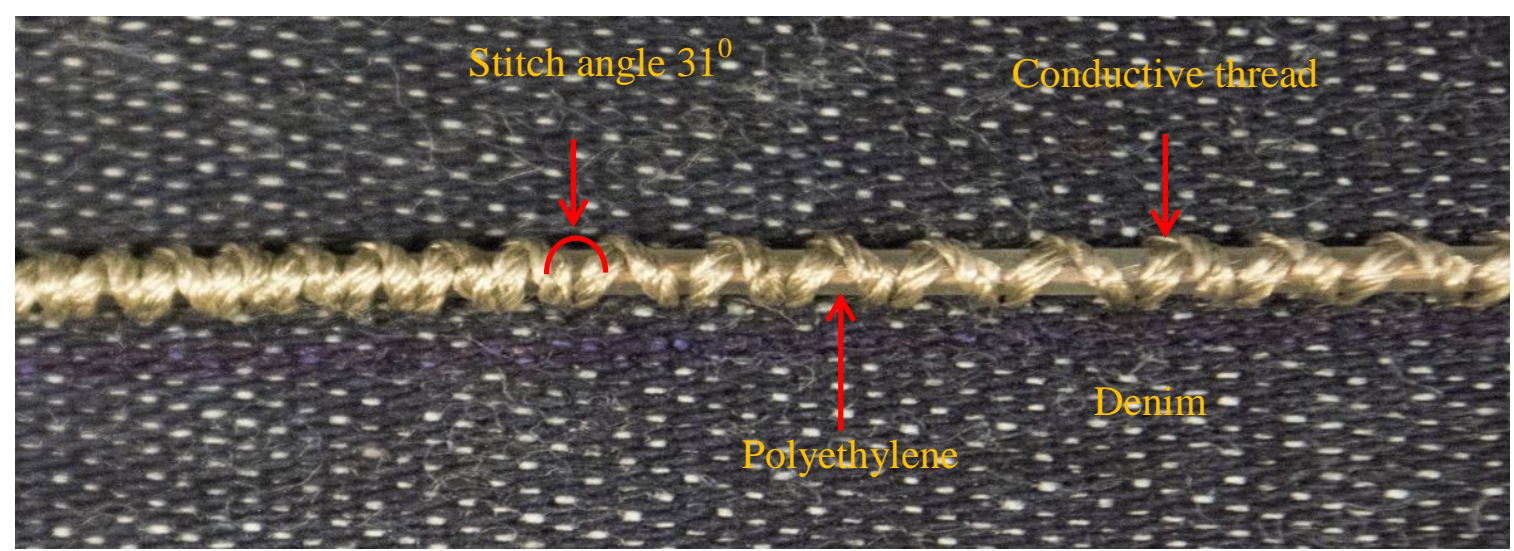

(c)

Figure 5.8 Fabricated stitched transmission line with stitch angles of (a) $85^{\circ}$ (b) $65^{\circ}$ and (c) $31^{0}$ 

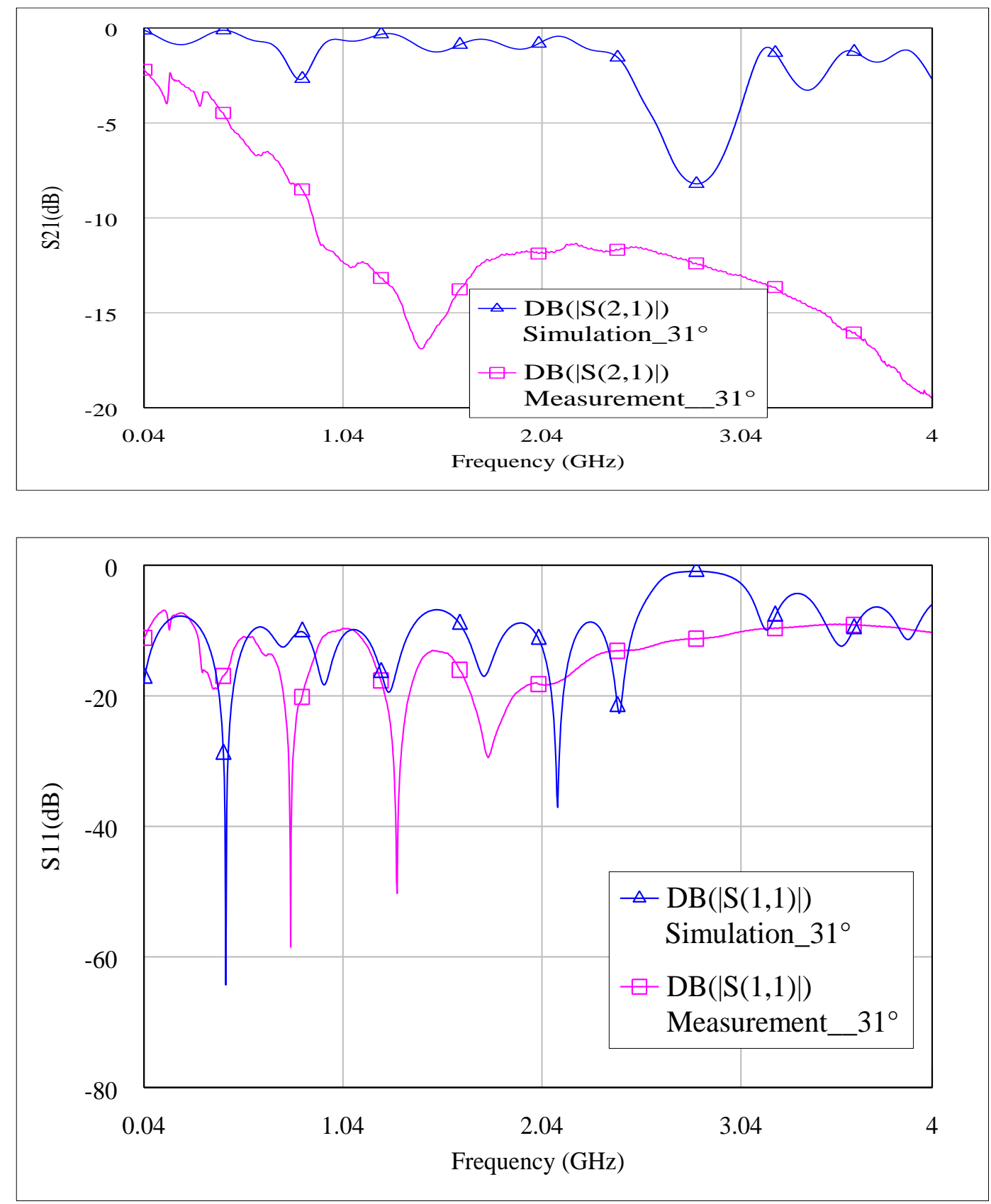

Figure 5.9 Measured S-Parameters of stitched transmission line with stitch angle and length at $31^{\circ}$ and $1.2 \mathrm{~mm}$ respectively 

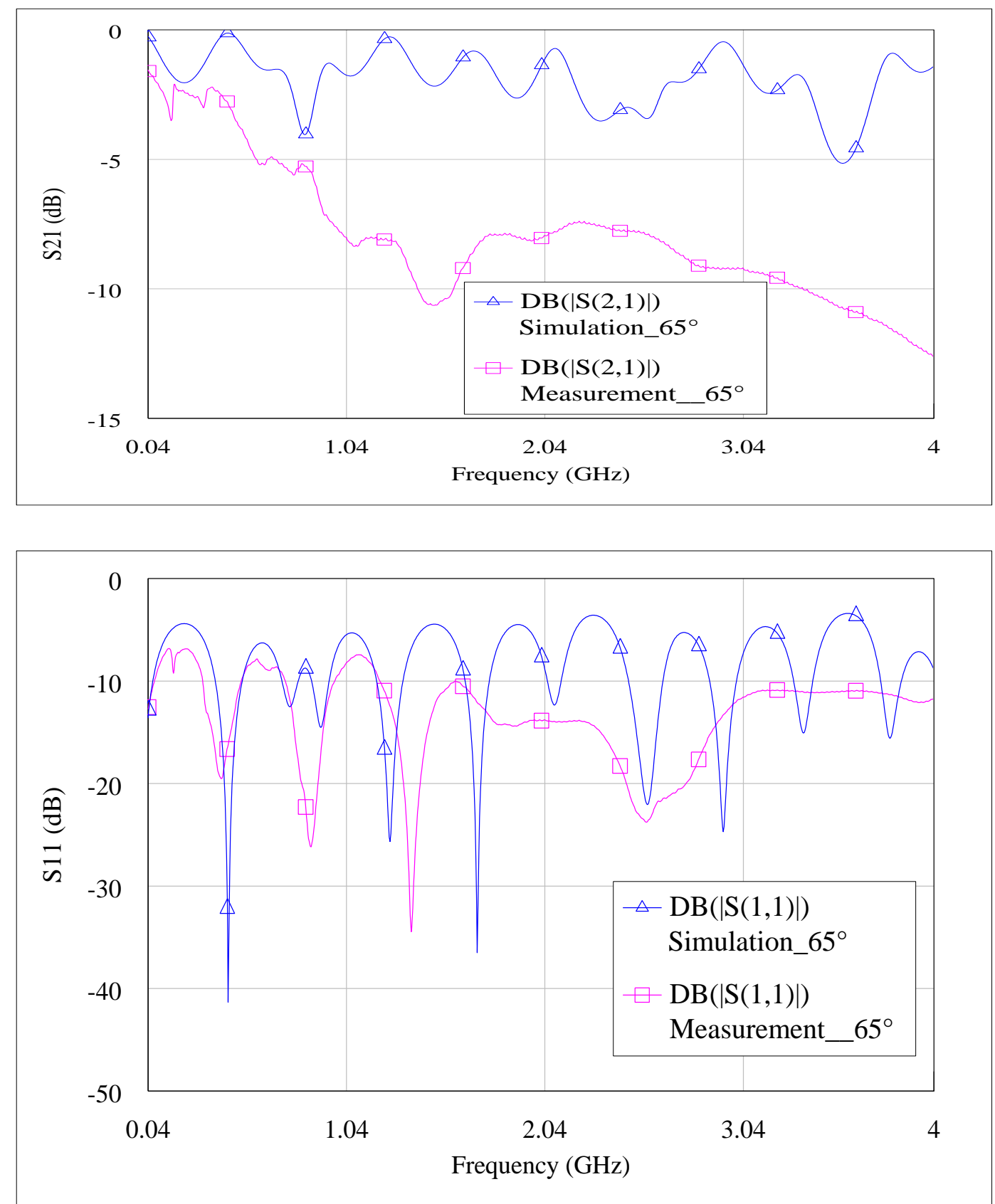

Figure 5.10 Measured S-Parameters of stitched transmission line with stitch angle and length at $65^{\circ}$ and $1.5 \mathrm{~mm}$ respectively 

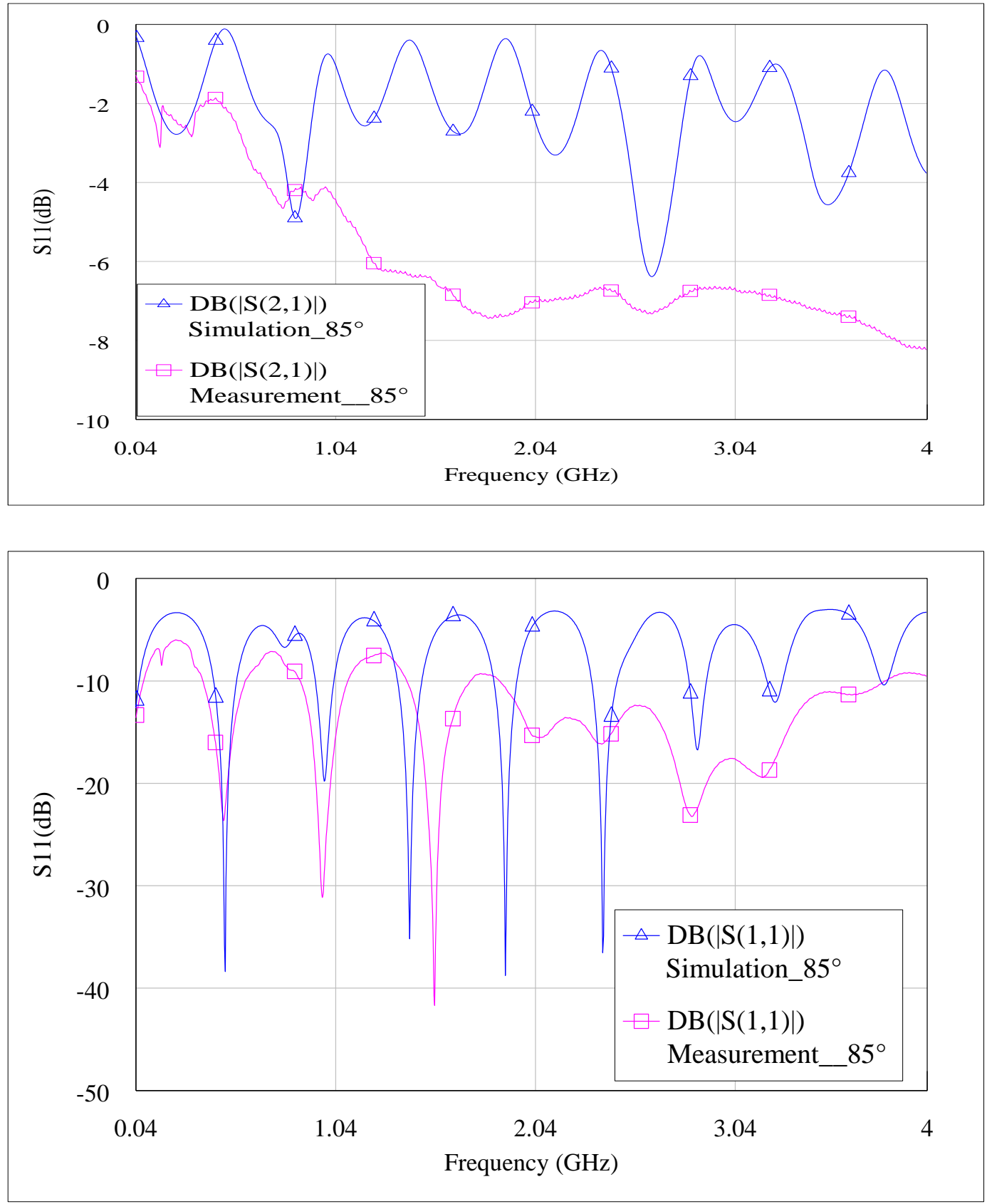

Figure 5.11 Measured S-Parameters of stitched transmission line with stitch angle and length at $85^{\circ}$ and $1.7 \mathrm{~mm}$ respectively 


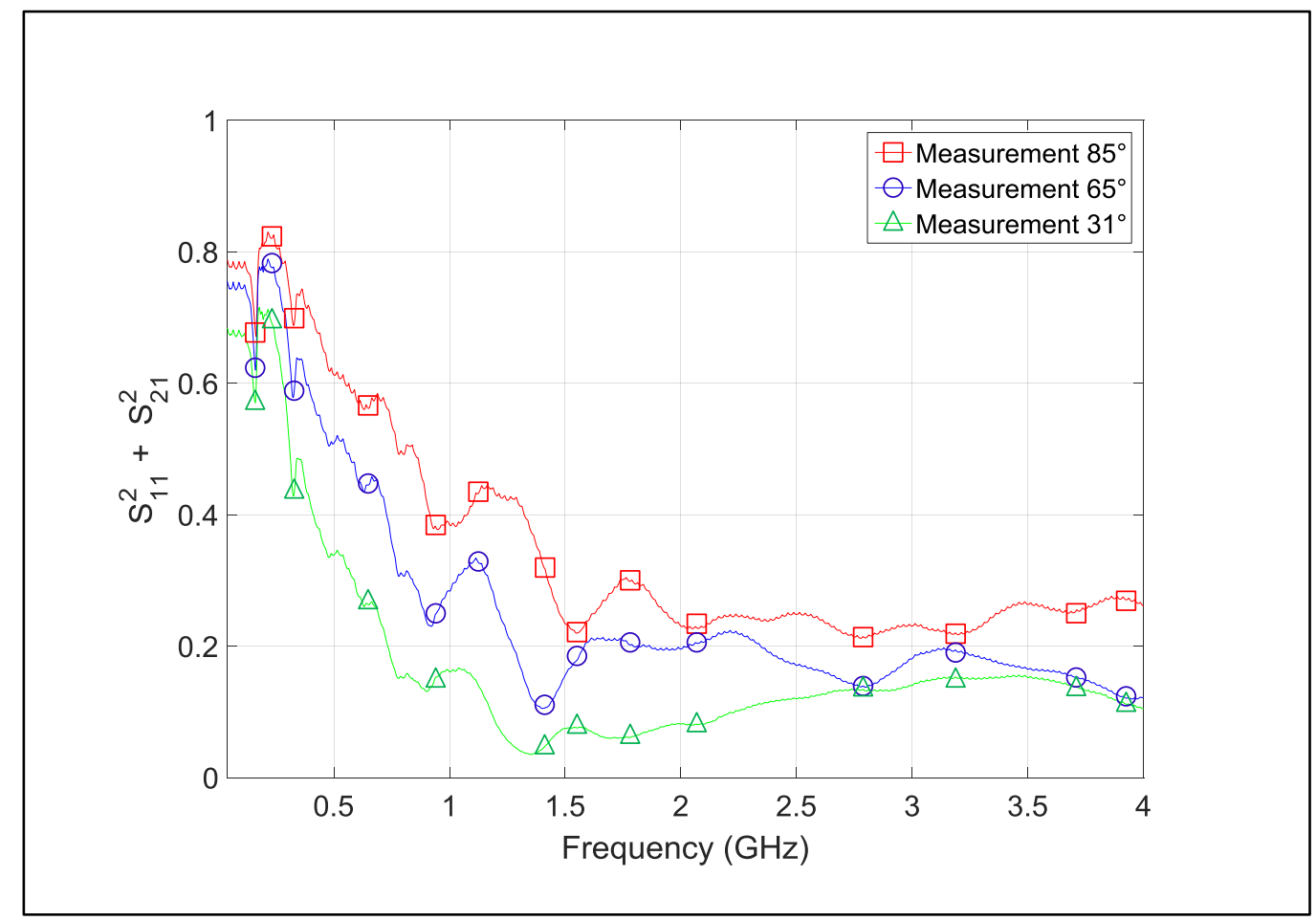

Figure 5.12 Plot of $\boldsymbol{S}_{\mathbf{1 1}}^{2}+\boldsymbol{S}_{\mathbf{2 1}}^{\mathbf{2}}$ against frequency with stitch angles $85^{\circ}, 65^{\circ}$ and $31^{\circ}$

\subsubsection{Stitched Transmission Line with Different Stitch Types}

Three different stitch types were used in the fabrication of the stitched wearable transmission line as presented in Fig.5.13 and 5.14, while Fig.5.15 and 5.16 presents the measured scattering parameters from 0.04 to $4 G H z$ and a plot of $\left(S_{11}^{2}+S_{21}^{2}\right)$ with frequency, for a straight $150 \mathrm{~mm}$ long stitched transmission line while Table 5.1 displays the measured DC Resistance for Double Overlock stitch, Flatlock stitch and Ric-Rac stitch.

The measured reflection coefficients $S_{11}$ are below $-9 d B$ for both Double Overlock and Flatlock stitch, and $-10 d B$ for Ric-Rac stitch in most of the operation band, while the transmission coefficients $S_{21}$ are better than $-13 d B$ for both Double Overlock and Flatlock stitch, and $-10.5 d B$ for Ric-Rac stitch. The Double Overlock stitch has a better shield coverage of the three stitch types. The DC losses are seen to be more for Double Overlock stitch and Flatlock stitch which both have higher stitch densities and a more complex geometry compared to Ric-Rac stitch for frequencies up to $1 \mathrm{GHz}$; the resistive losses are dominant at lower frequencies, while radiation losses are more dominant at higher frequencies. These DC losses are mainly influenced by the stitch geometry which is characterised by the hierarchical 
structure of the stitch and density which is also characterised by the distance between individual stitches in a column or row. From Fig. 5.16, the Ric-Rac stitch has fewer losses of the three for frequencies up to $1 \mathrm{GHz}$. However beyond that up to $2.4 \mathrm{GHz}$ and above that, the Flatlock stitch and the Double Overlock stitch have fewer losses respectively. The choice of the best stitch to use will ultimately depend on the range of the frequency of transmission.

TABLE 5.1 Measured DC Resistance for Double Overlock stitch, Flatlock stitch, and RicRac stitch

\begin{tabular}{cc}
\hline Stitch Type & Measured DC Resistance of Shield $(\Omega)$ \\
\hline Double Overlock stitch & 30.3 \\
Flatlock stitch & 16.7 \\
Ric-Rac stitch & 22.4 \\
\hline
\end{tabular}

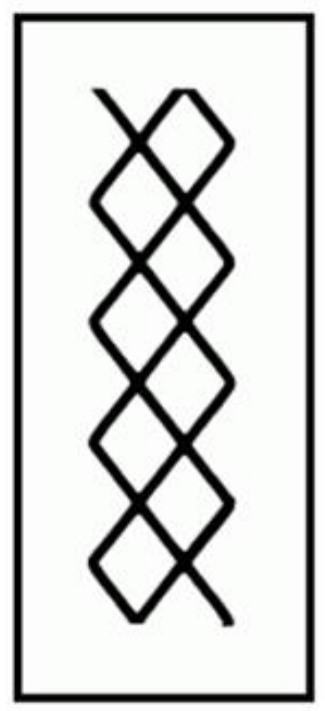

(a)

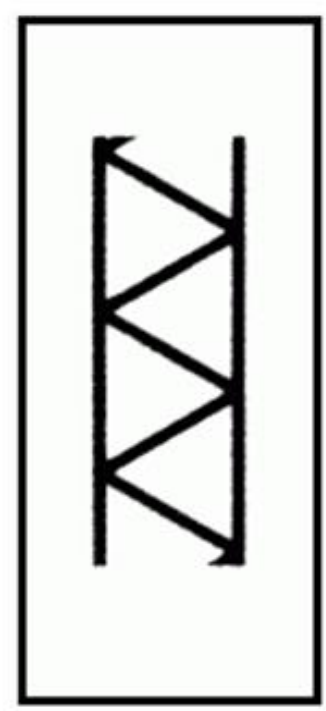

(b)

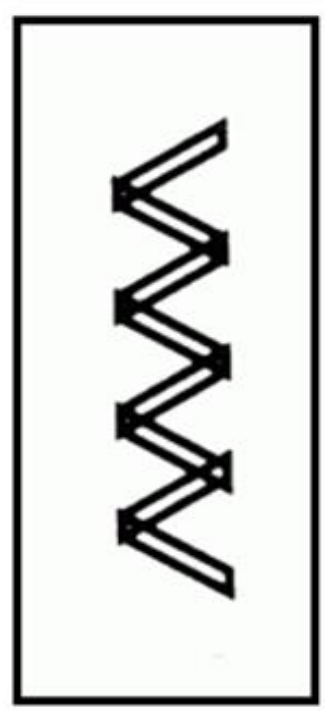

(c)

Figure 5.13 Three different stitch types used (a) Double Overlock stitch (b) Flatlock stitch and (c) Ric-Rac stitch 


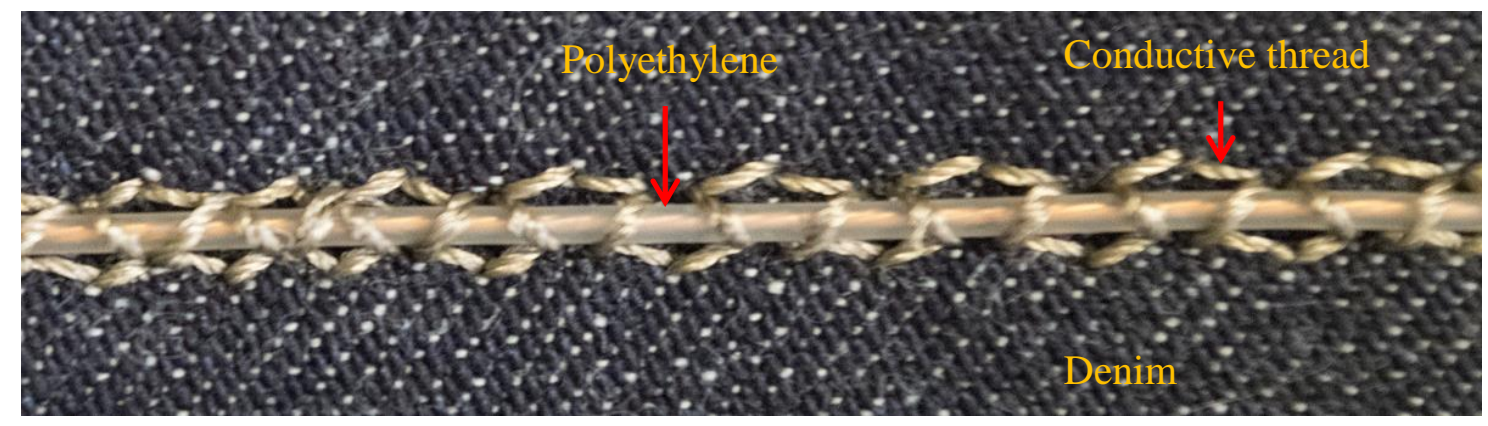

(a) Double Overlock

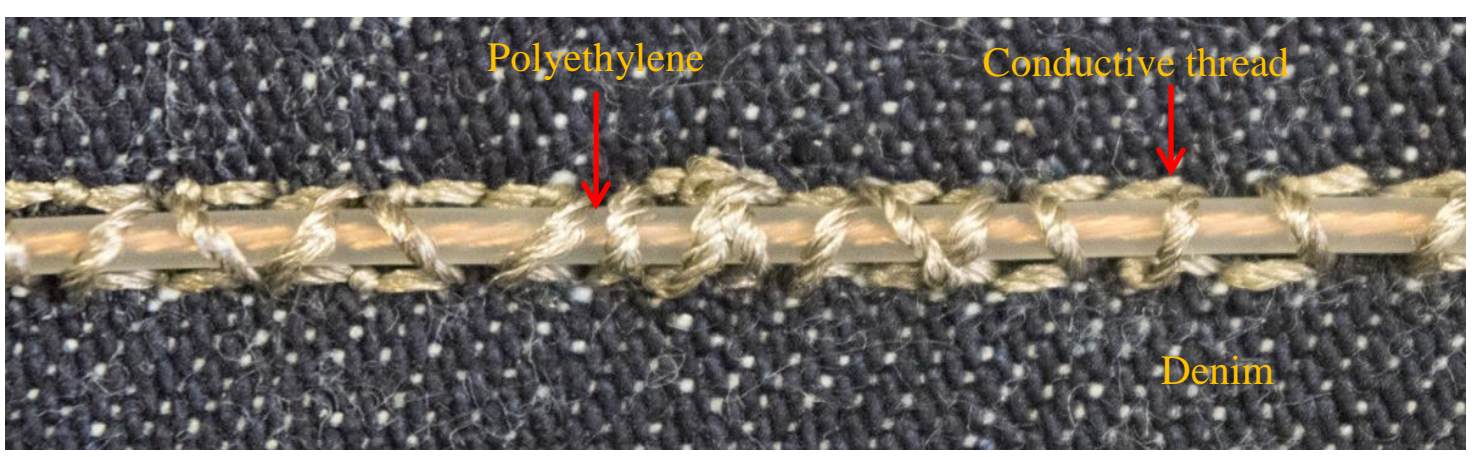

(b) Flatlock stitch

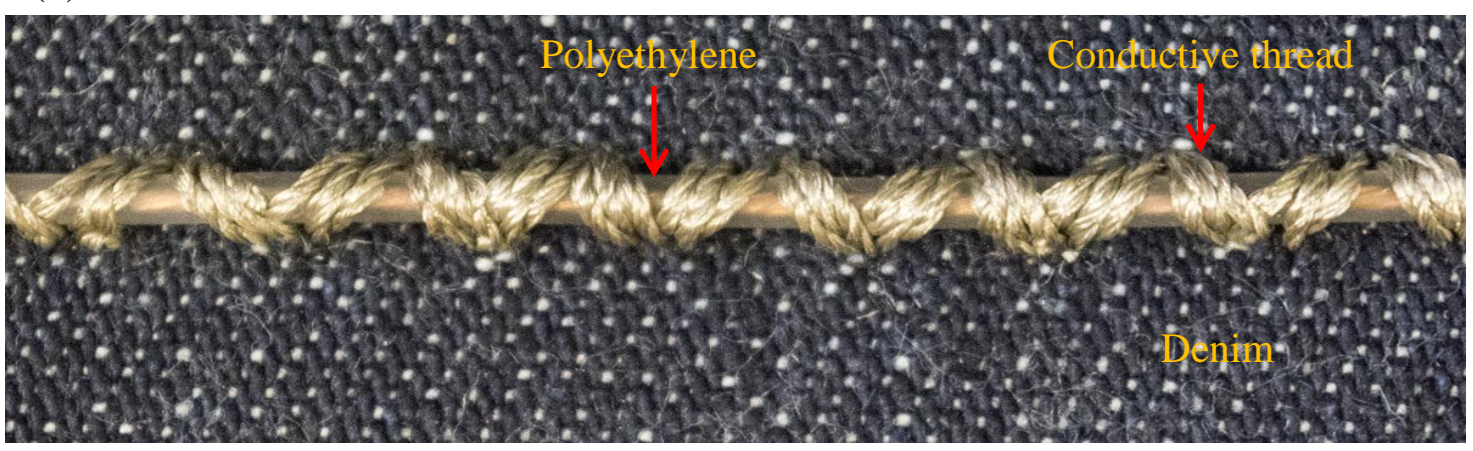

(c) Ric-Rac stitch

Figure 5.14 Zoomed in view of constructed stitched transmission line with three different stitch types 

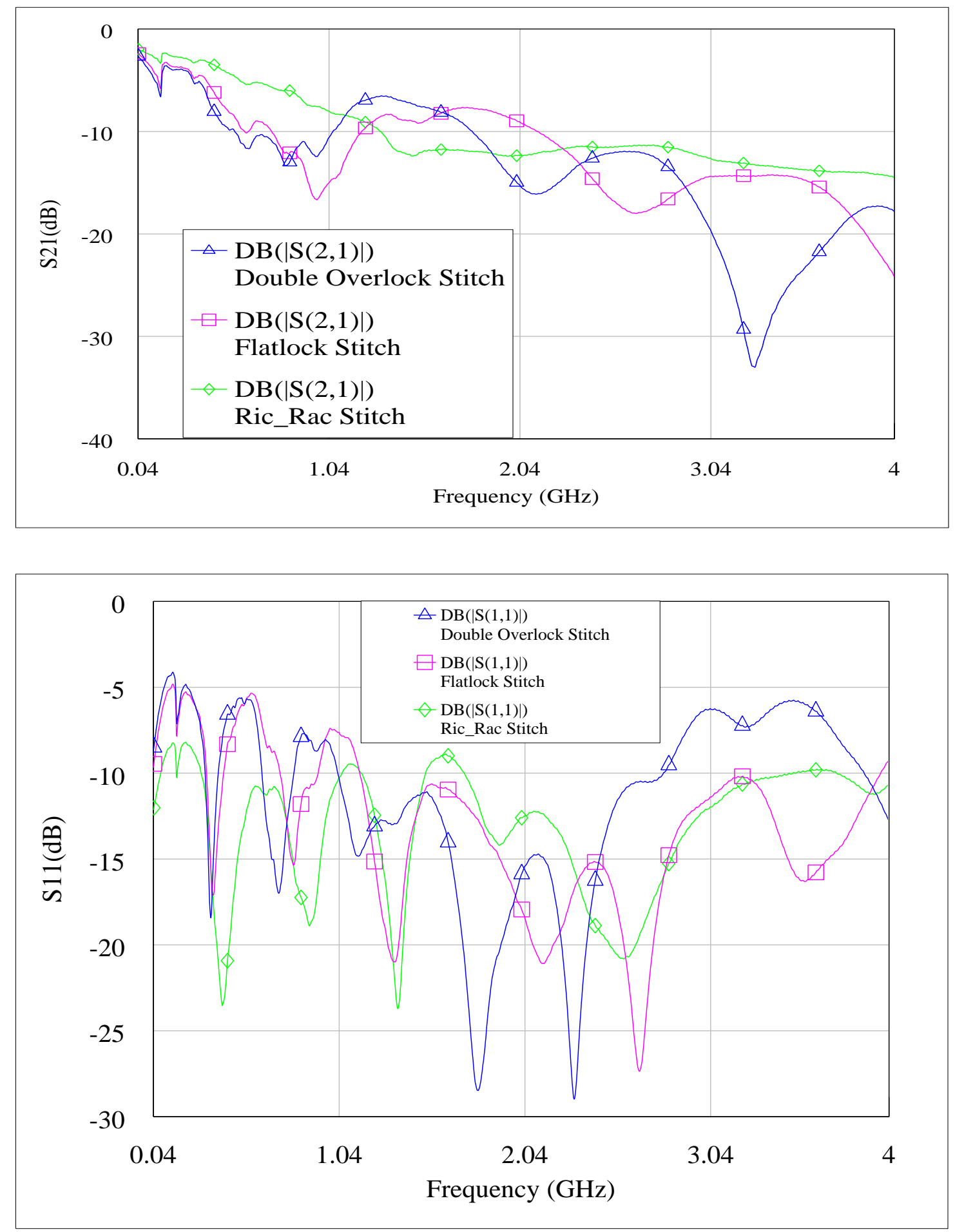

Figure 5.15 Measured S-Parameters of stitched transmission line with three different stitch types 


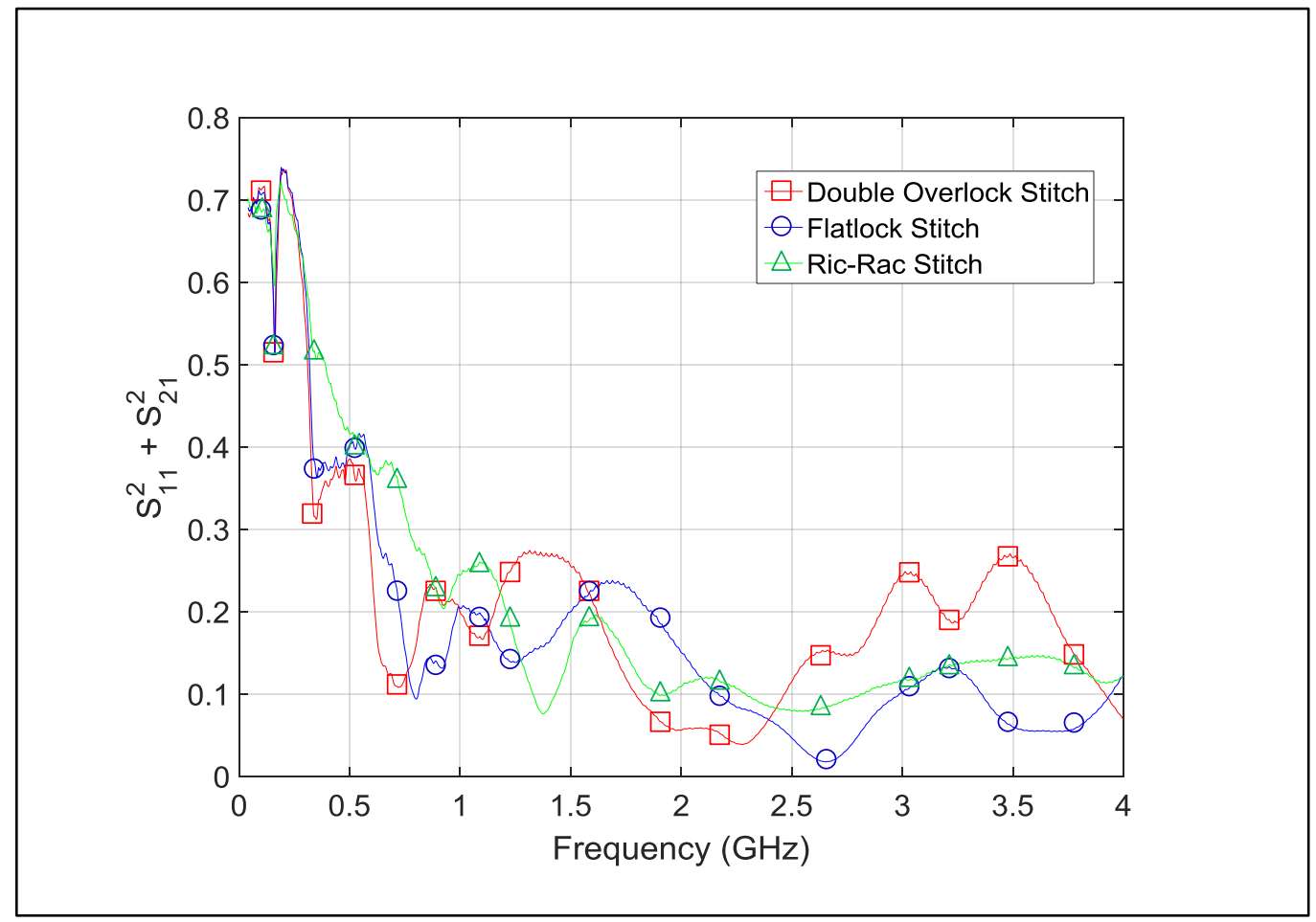

Figure 5.16 Plot of $\boldsymbol{S}_{\mathbf{1 1}}^{\mathbf{2}}+\boldsymbol{S}_{\mathbf{2 1}}^{\mathbf{2}}$ against frequency with three different stitch types

\subsubsection{Stitched Transmission Line with Different Curved Bending Angles}

The convenience, robustness, flexibility and operational reliability of the stitched transmission line for various bending positions are very important, as it is stitched or embedded into apparels and worn by humans. The robustness of the stitched transmission line was put to test by bending it through curved angles of $90^{\circ}$ and $180^{\circ}$. The RG174 braided coaxial cable used in the fabrication of the stitched transmission line has a bend radius 10 times its diameter [5.1]. A comparable technique used by $\mathrm{Xu}$ et al. in [5.2] for a wearable textile shielded stripline used for signal transmission, where the robustness of the stripline was put to test within a frequency range of $0.01 \mathrm{GHz}-8 \mathrm{GHz}$ in terms of its propagation characteristics was seen to maintain consistent propagation characteristics when bent through angles of $90^{\circ}$ and $180^{\circ}$.

Measurements on the fabricated stitched transmission line with stitch length of $2 \mathrm{~mm}$ were carried out for curved angles of $90^{\circ}$ and $180^{\circ}$, with a bending radius of $75 \mathrm{~mm}$ as illustrated in Fig.5.17, with the scattering parameters from 0.04 to $4 \mathrm{GHz}$ and a plot of $\left(S_{11}^{2}+S_{21}^{2}\right)$ with 
frequency presented in Fig.5.18 and 5.19. Compared with the planar stitched wearable transmission line, a much better $S_{21}$ is observed at $180^{\circ}$ curved bending angle for frequencies below 2.1GHz. Discontinuity such as a bend and periodic apertures are responsible for radiation losses [5.3]. However, changes in current locations is responsible for the increase in radiation from bent wires and not changes in current distribution or reflections at corners [5.4]. It is quite difficult in identifying the radiation from the bend as the major source of radiation because of the sparse nature of the shield of the stitched transmission line. It is also important to note here that level of loss or attenuation for a braided coaxial cable increases when bent sharply even if the bend is within manufacturers recommended bend radius. However, for the designed stitched transmission line, results presented are for a curved bend angles as a result of gradual bending of the stitched transmission line angle and not a sharply bent angle.

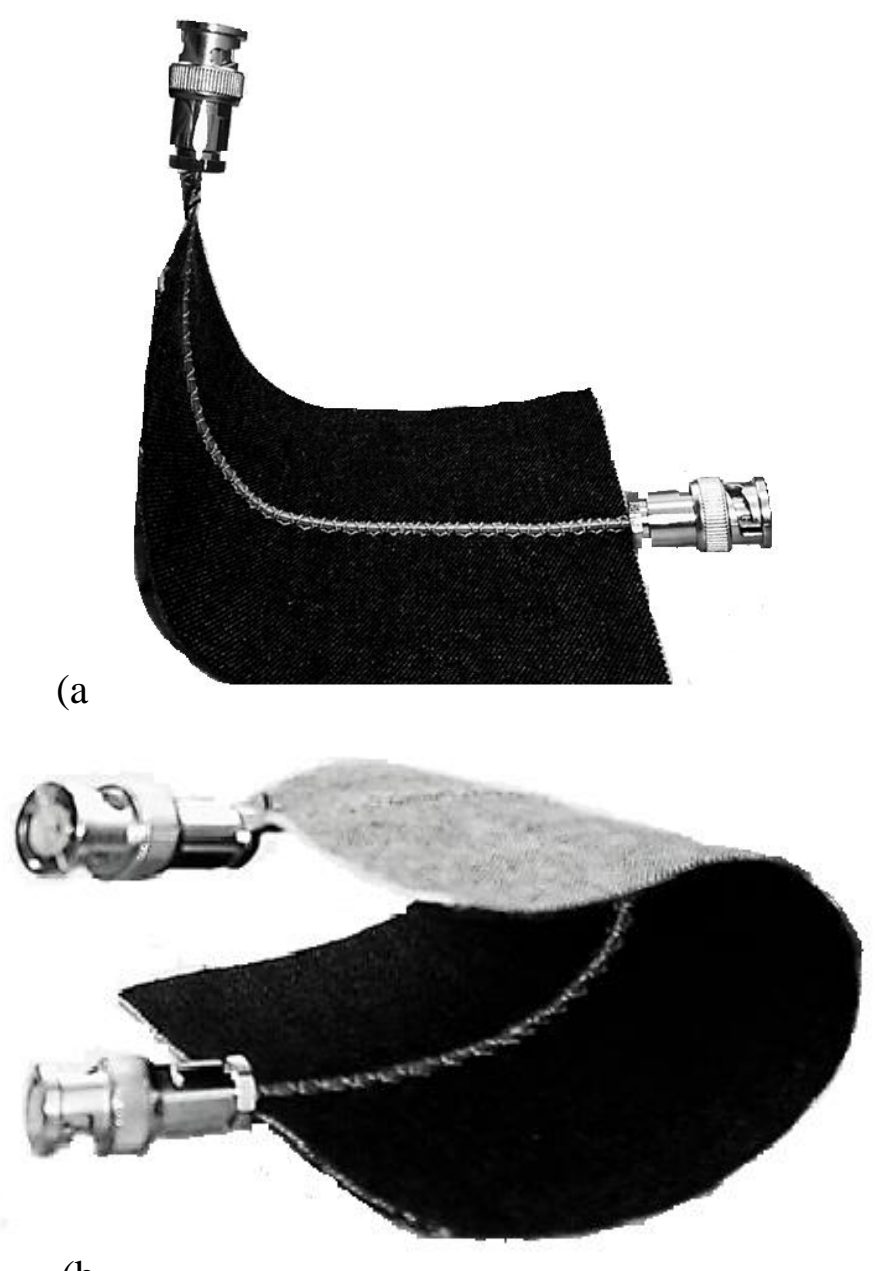

$(\mathrm{b}$

Figure 5.17 Stitched transmission line with different curved bending angles at (a) $90^{\circ}$ (b) $180^{\circ}$ 

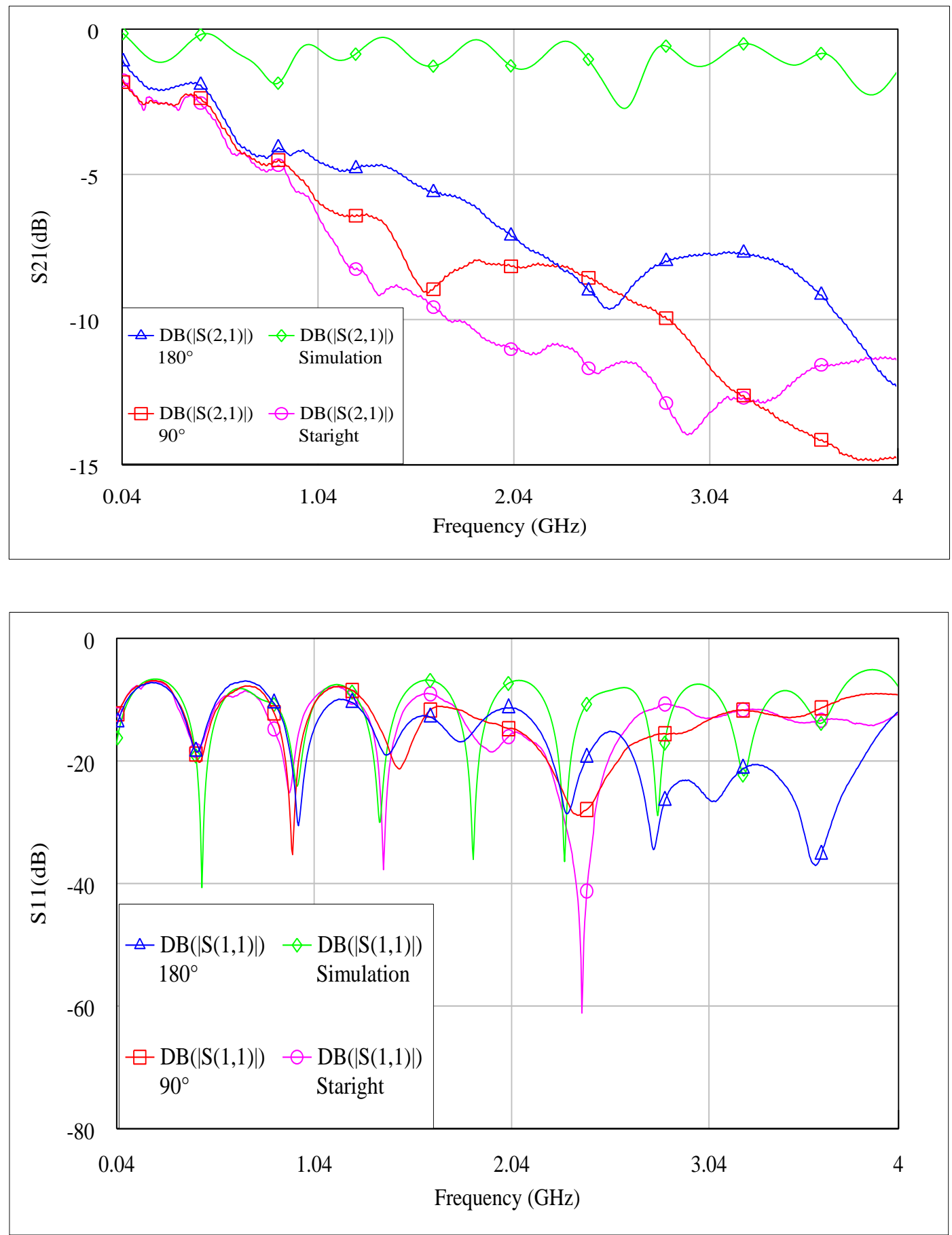

Figure 5.18 Measured S-Parameters of stitched transmission line with two different bending conditions and a straight stitched line compared with a straight simulated line 


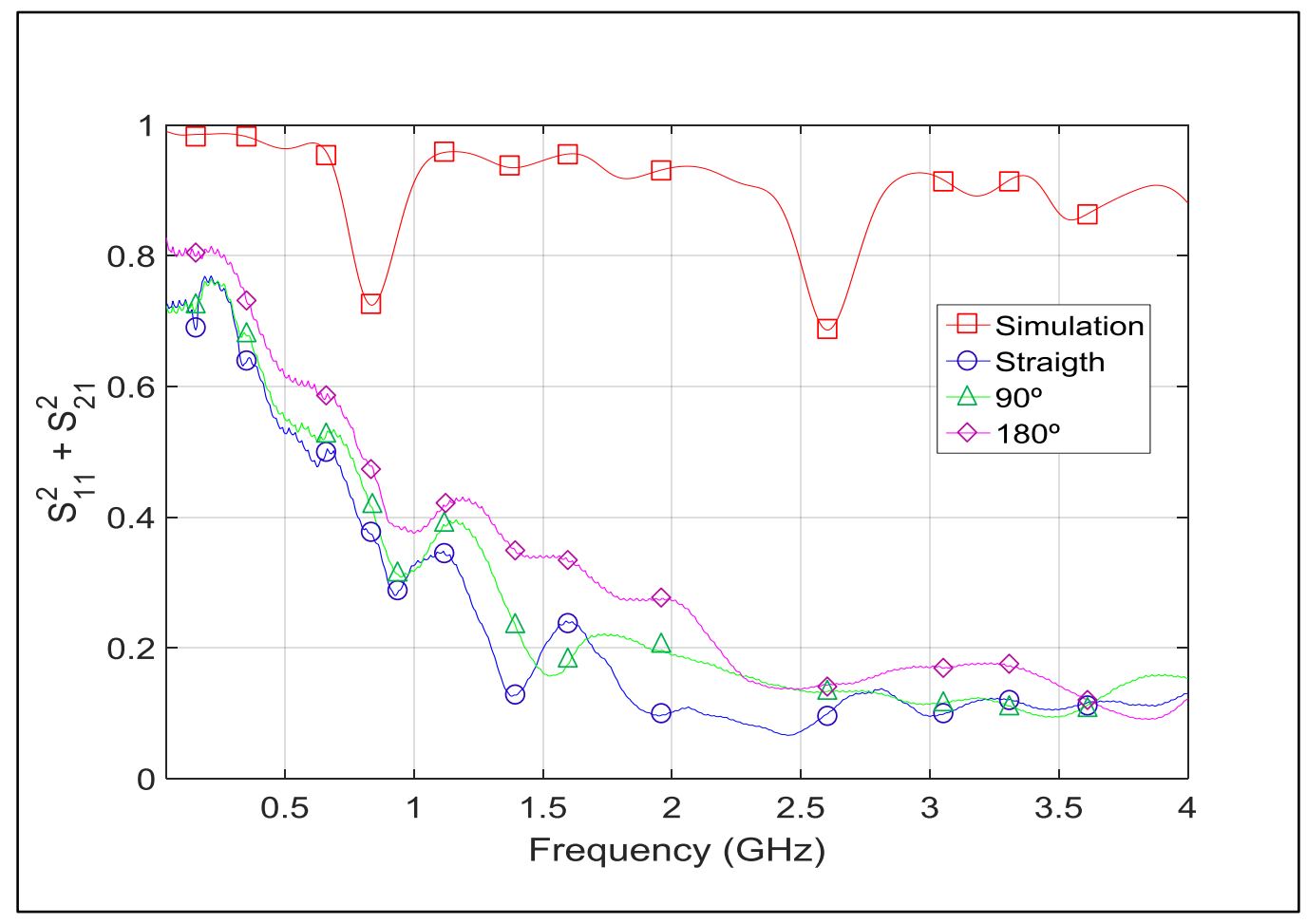

Figure 5.19 Plot of $\boldsymbol{S}_{\mathbf{1 1}}^{2}+\boldsymbol{S}_{\mathbf{2 1}}^{2}$ against frequency with two different bending conditions and a straight stitched line compared with a straight simulated line

\subsubsection{Stitched Wearable Transmission Line Subjected to Washing Cycles}

The exposures to rain, sweat, dust, and dirt can affect the performance of the stitched transmission line. For the stitched transmission line to be used in smart textile applications, good wash-ability properties are critical. The domestic washing behavior of the wearable stitched wearable transmission line with a stitch length of $2 \mathrm{~mm}$ was evaluated after subjecting it to washing cycles using Bio Persil detergent washing powder in a Hotpoint washing machine. Two samples of the stitched transmission line were produced. While measurements were carried out on the first without being washed, the second was subjected to washing cycles without the connectors before measurements, and both results compared. The aim here is to ascertain the deterioration of frequency dependence of the transmission lines when the fabrics are subjected to washing cycles for frequencies up to $4 \mathrm{GHz}$. The stitched transmission lines under study here are made up of a stripped RG174, stitched on a Denim material. The washed stitched transmission line was subjected to a standard 
$60^{\circ} \mathrm{C}$ cycle. The DC resistance as well as the scattering parameters of the line were measured before and after washing.

The measured scattering parameters from 0.04 to $4 \mathrm{GHz}$ before and after washing cycles are shown in Fig.5.20, while the measured DC resistance is given in Table 5.2. From Table 5.2 the DC Resistance is seen to increase from $16.9 \Omega$ before washing to $22.8 \Omega$ after washing as expected. This was expected because as temperature changes, the dimensions of the conductor will change as it expands or contracts. Although, these changes in resistance cannot be explained by a change in dimensions due to thermal expansion or contraction, for a given size of conductor the change in resistance is mainly due to a change in the resistivity of the material and is caused by the changing activity of the atoms that make up the material. However, a much plausible reason for the increase in the resistance of the stitched transmission line after washing can be attributed to the loss of some metal fibers due to abrasion impacts in the washing machine. It was also observed that the conductive threads appeared loose and flailed (see Fig.5.22) after the wash especially at the ends of the transmission line which also made it quite difficult to connect to connectors.

The DC losses were seen to increase in the washed transmission line compared to the unwashed stitched transmission line at lower frequencies. Interestingly, the scattering parameters look much better with the washed line, which is quite good for wearability applications. Two hypothesis are proposed here as to why this could be happening; first, the washing off of the oil that sticks to the stitched transmission line during washing. However, this could also lead to the swelling of the thread when it absorbs water through pre-existing cracks and defects in the silver plating causing an increasing amount of cracks and defects, and secondly, the slight change in the tension within the threads. Generally, textile materials have viscoelastic behavior [5.5], internal tensions relieve over time and the geometry may also change due to washing [5.6]. Equally, the washing of the stitched transmission line at high temperatures can affect the conductive thread by tarnishing the coated silver as silver easily gets tarnished [5.7] when exposed to moisture and airborne acids. The tarnish which can reduce the surface conductivity of the silver is a hard insulating oxide layer that forms on the silver surface. The extent to which this can be reduced depends on the thickness of tarnish. Poor water quality, in particular, sulphur, high Fluoride and low $\mathrm{pH}$ will react strongly with silver could also affect its conductivity and shielding performance. The dielectric also has an effect on some types of polyethylene can absorb moisture more readily than others. A plot of $\left(S_{11}^{2}+S_{21}^{2}\right)$ with frequency in Fig.5.21 further confirms results obtained earlier in Fig.5.20. 
TABLE 5.2 DC resistance of the shield of stitched transmission line before and washing

\begin{tabular}{|c|c|c|c|c|c|c|c|}
\hline \multicolumn{3}{|c|}{ Stitched transmission line } & \multirow{2}{*}{$\begin{array}{c}\text { Washing } \\
\text { Type }\end{array}$} & \multirow{2}{*}{$\begin{array}{c}\text { Washing } \\
\text { Temperature } \\
\left({ }^{\circ} C\right) \\
\end{array}$} & \multirow{2}{*}{$\begin{array}{c}\text { Detergent } \\
\text { used }\end{array}$} & \multirow{2}{*}{$\begin{array}{c}\text { Resistance } \\
\text { before washing } \\
(\Omega) \\
\end{array}$} & \multirow{2}{*}{$\begin{array}{c}\text { Resistance after } \\
\text { washing } \\
(\Omega) \\
\end{array}$} \\
\hline Stitch & Stitch & Stitch & & & & & \\
\hline Tension & Width & Length & $\begin{array}{l}\text { Machine } \\
\text { Wash }\end{array}$ & 60 & Bio Persil & 16.9 & 22.8 \\
\hline 4 & 2 & 2 & & & & & \\
\hline
\end{tabular}
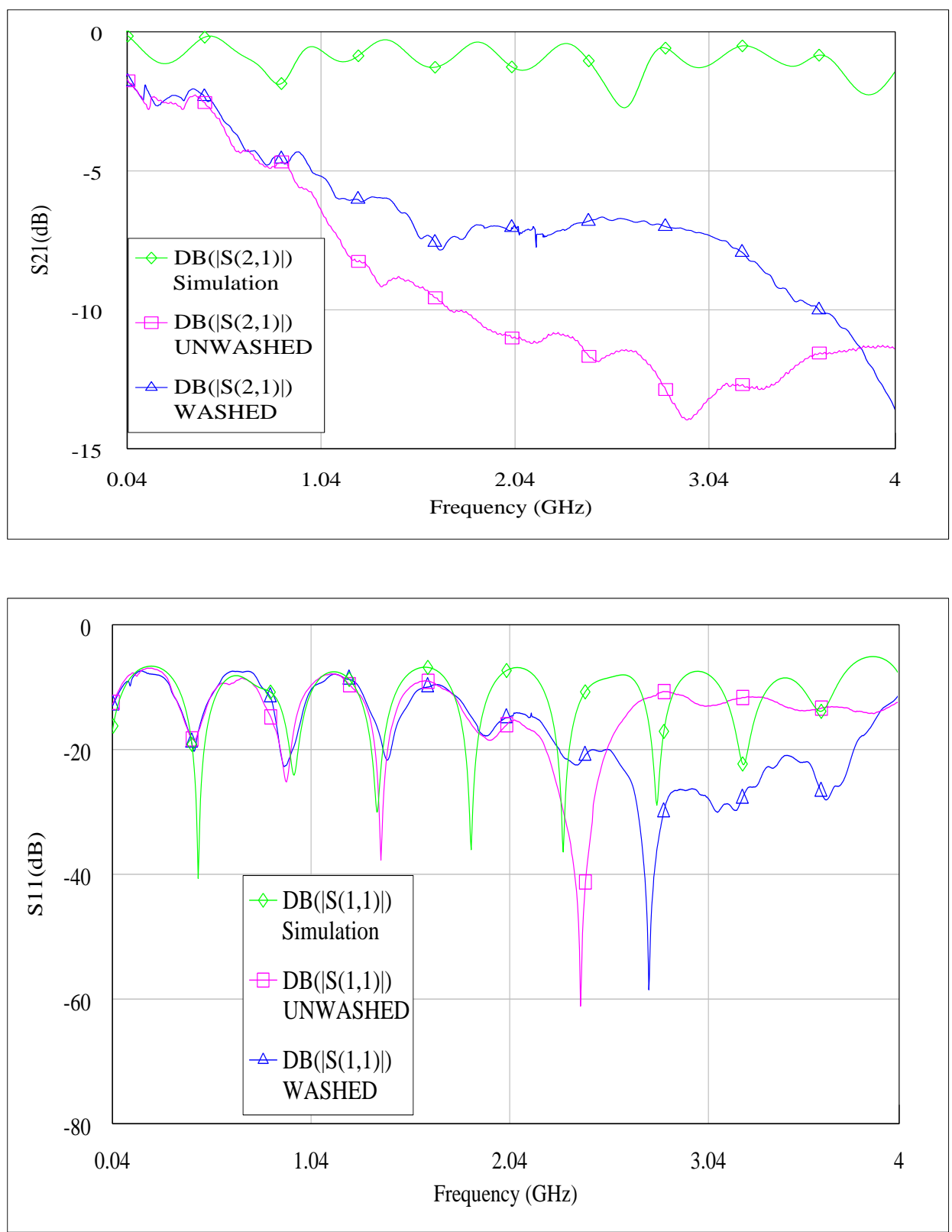

Figure 5.20 Measured S-Parameters of Washed and Unwashed stitched transmission line 


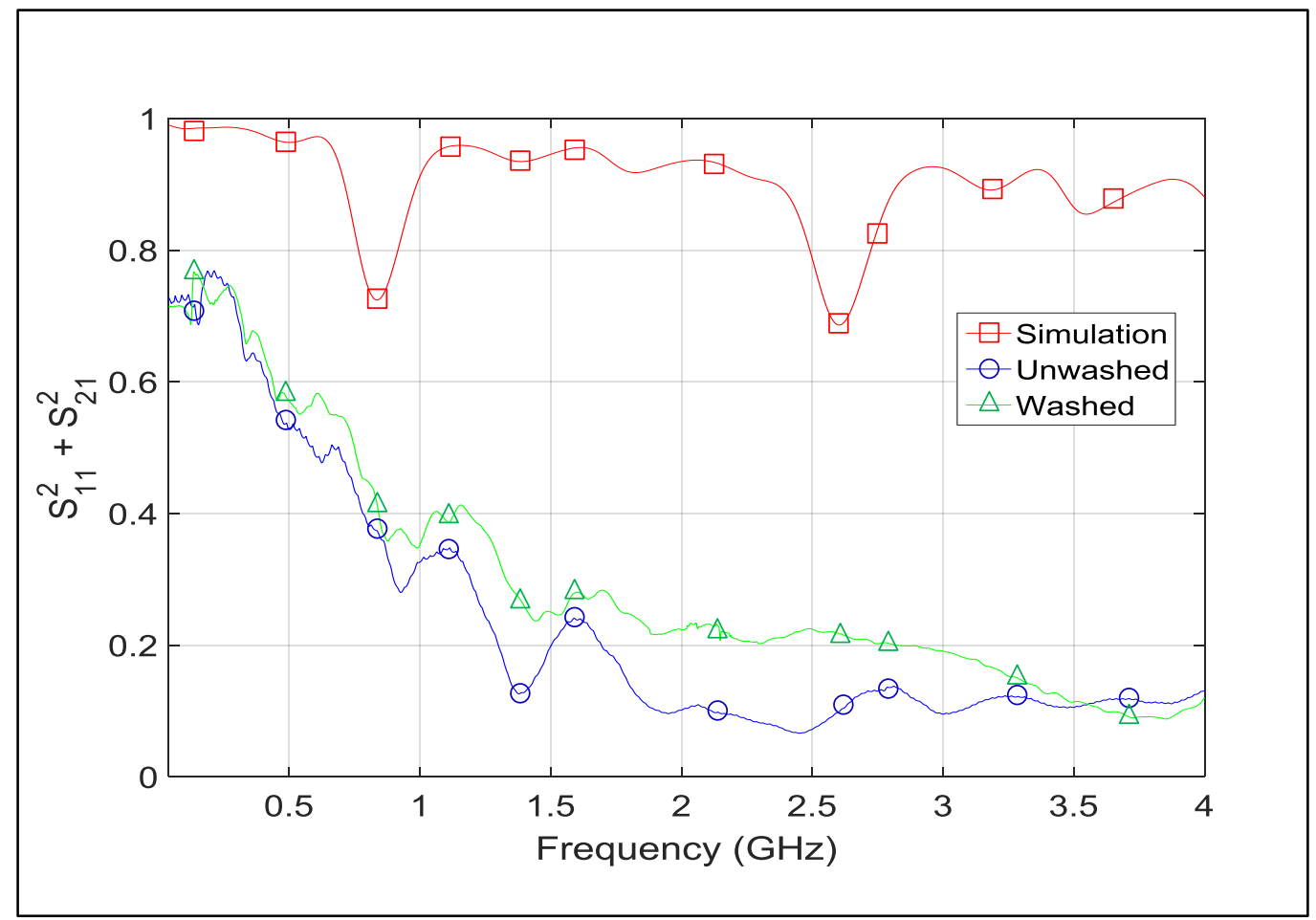

Figure 5.21 Plot of $\boldsymbol{S}_{\mathbf{1 1}}^{\mathbf{2}}+\boldsymbol{S}_{\mathbf{2 1}}^{\mathbf{2}}$ against frequency with two different bending conditions

Scattering electron microscope (SEM) images were taken with the JEOL JSM-7800F FESEM, to visually investigate the condition of the conductive threads before and after washing. Prior to using the Scattering electron microscope, the two samples were sputter coated for 60 seconds with Gold palladium ( $\mathrm{Au} / \mathrm{Pd}$ ) using Quorum Q150R, which is a compact rotarypumped coating system suitable for SEM sputtering with non-oxidising (noble) metals and for carbon coating SEM specimen for EDS (Energy-dispersive X-ray spectroscopy) and WDS (wavelength dispersive X-ray spectroscopy) [5.8]. Fig. 23 and 24 (a)-(e), shows the scanned images of the stitched transmission line, EDS map spectrum with images showing the average composition in weight $\%$ (wt. \%) and the statistical error displayed as $\sigma$ (weight $\%$ sigma) for the calculated weight and the X-ray element maps showing presence of carbon, silver and oxygen. For the unwashed sample, the silver is seen to be evenly distributed with some fissures on it. The unwashed sample also contains carbon, silver and oxygen with some trace of copper and calcium which are both below detection limits. With the washed sample, silver is also seen to be evenly distributed. The fissures on the conductive thread appear to be 
more on the washed sample which shows the continuous silver coating to be more delaminated by washing. The washed sample also contains carbon and silver, with some trace amount of copper and calcium which all appear to have more average composition in weight $\%$ compared to the unwashed sample.

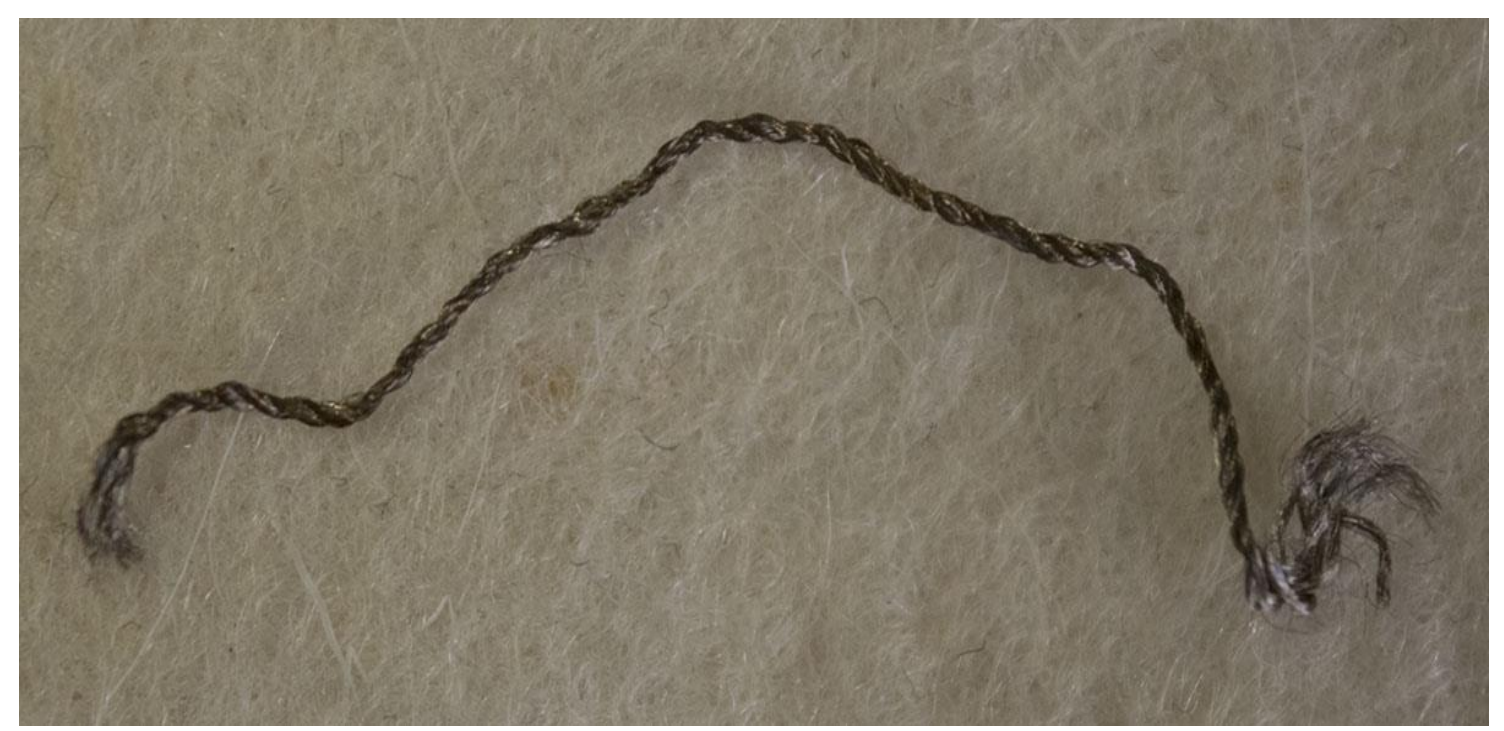

Figure 5.22 Cutoff section of the washed thread from the stitched transmission line 


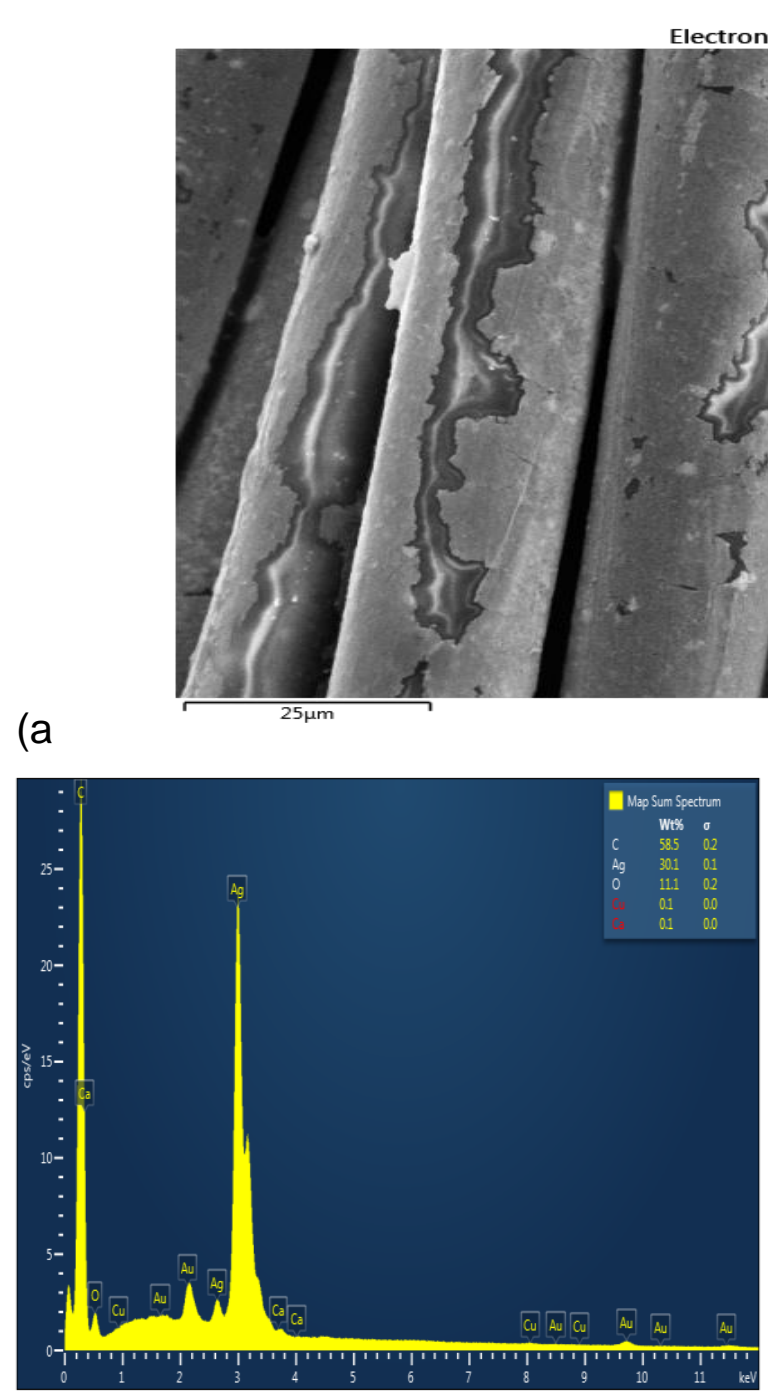

(b)

Ag $\operatorname{L\alpha 1}$

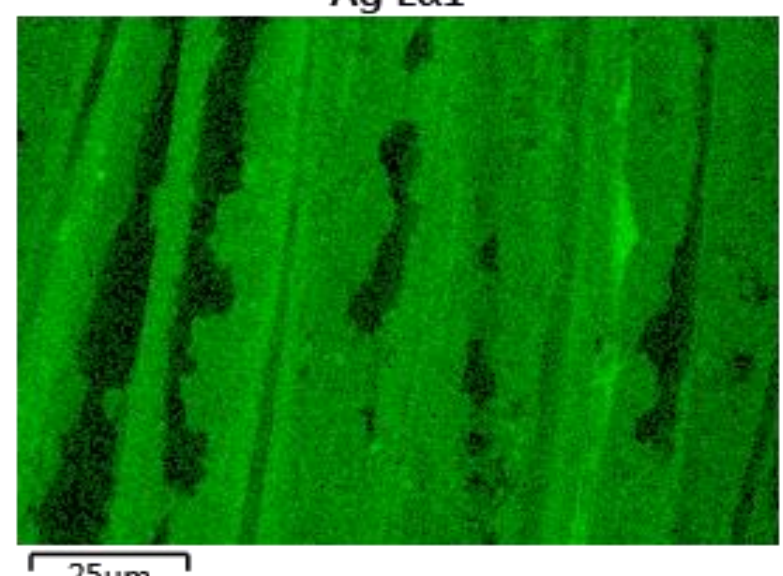

$25 \mu \mathrm{m}$

(d

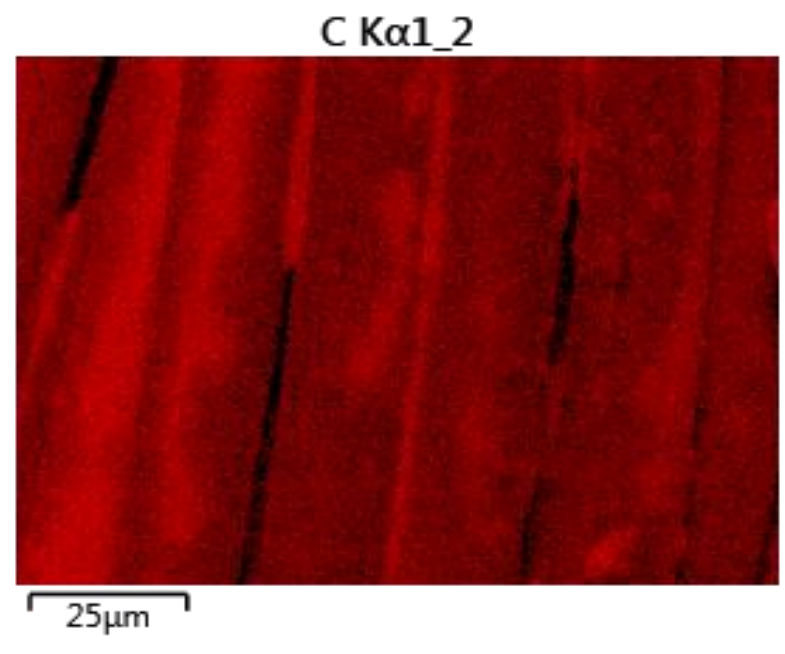

(c)

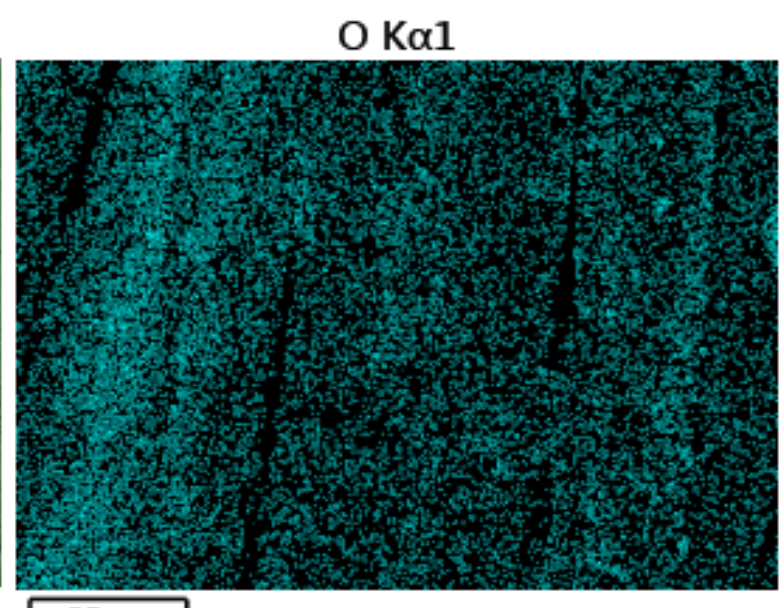

$\longdiv { 2 5 \mu \mathrm { m } }$

(e)

Figure 5.23 Scanned image of unwashed stitched transmission line with (b) showing the composition of the elements, while (c)-(e) colors depicting the presence of the elements 


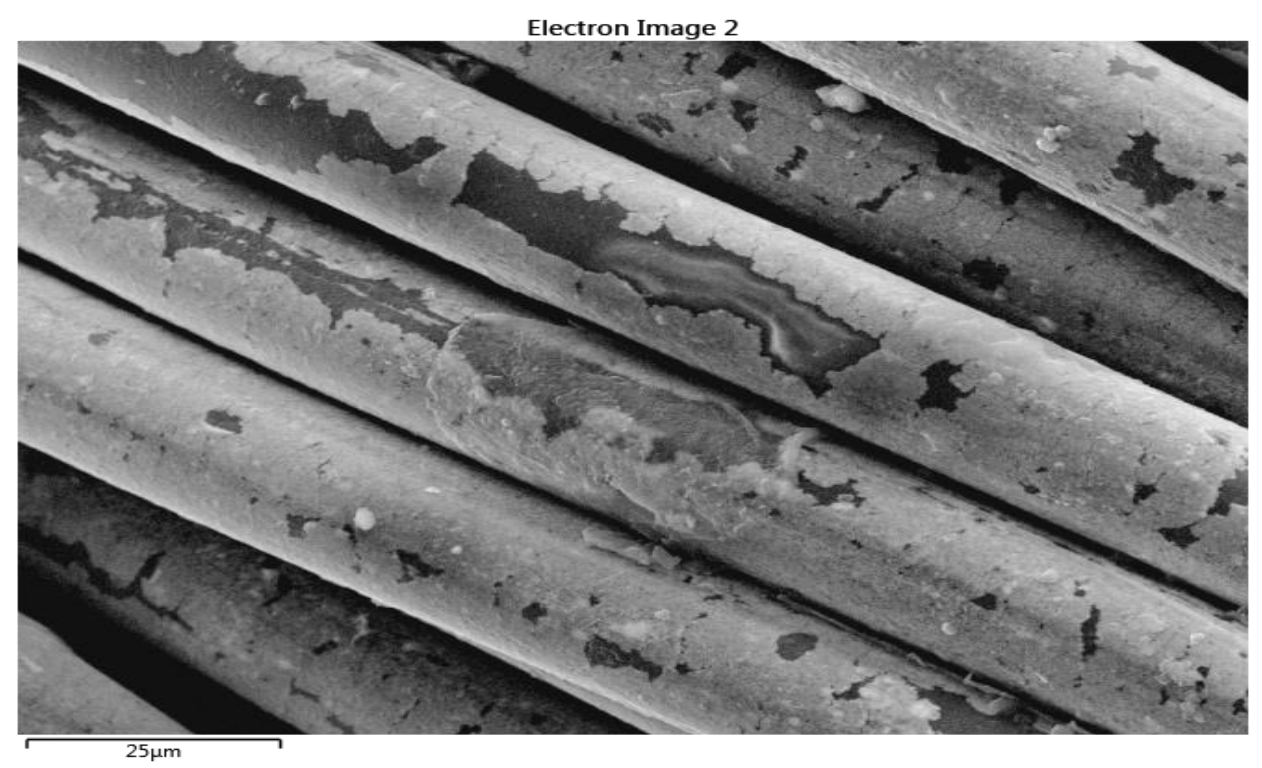

(a)

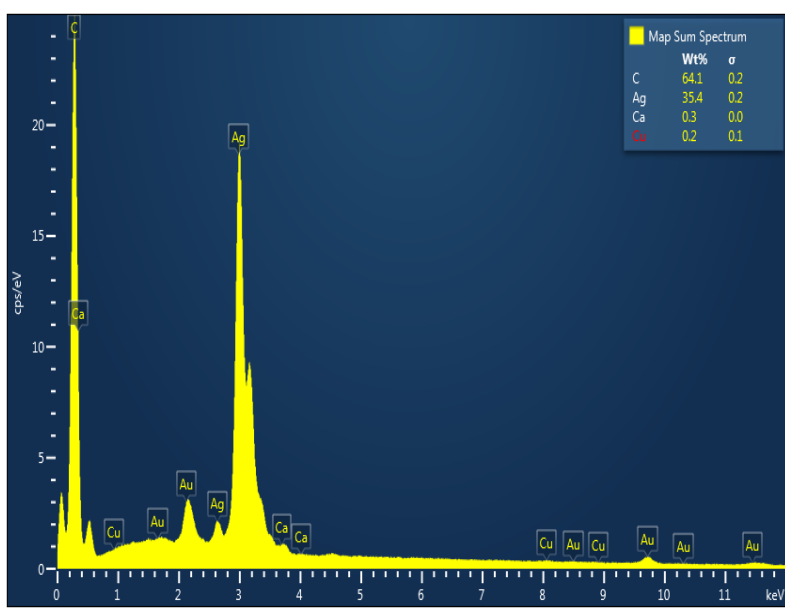

$\mathrm{C} K \alpha 12$

(b)

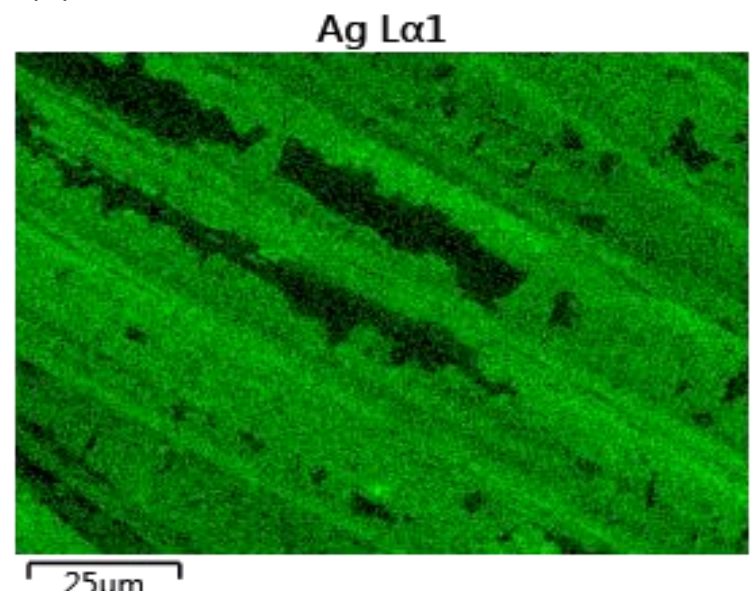

(d)

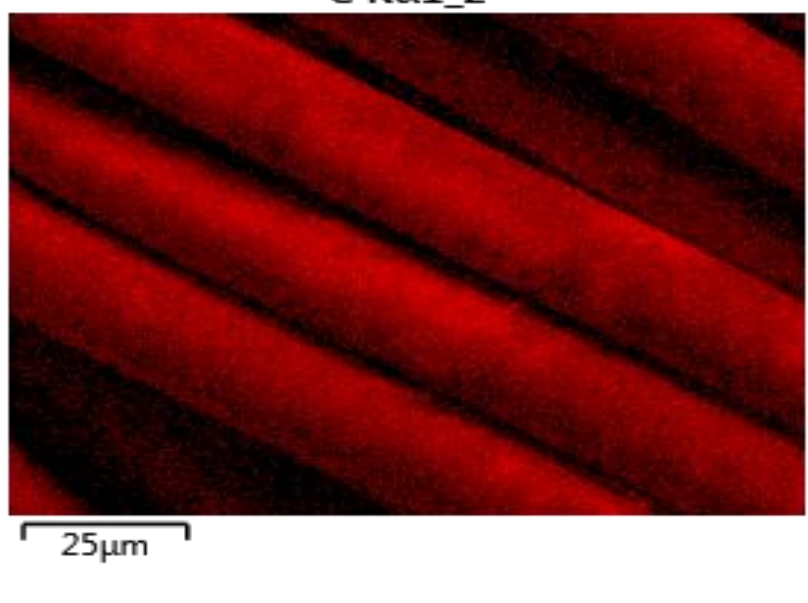

(c)

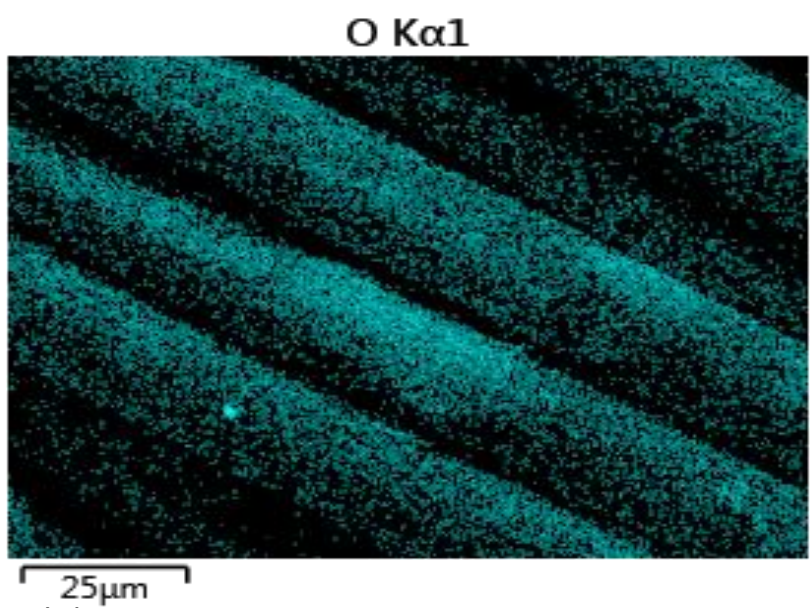

(e)

Figure 5.24 Scanned image of washed stitched transmission line with (b) showing the composition of the elements, while (c)-(e) colors depicting the presence of the elements 


\subsubsection{Stitched Transmission Line On-body Measurements}

Since the stitched transmission line is wearable, there is a requirement to carry out on-body measurements on the human body. A stitch transmission line with a stitch length of $1.2 \mathrm{~mm}$ was used for this purpose. Fig. 5.25 depicts the proximity of the stitch transmission line to the human body during measurement, while Fig. 5.26 depicts the measured stitched transmission line when placed very close to the human body. The stitched transmission line was flattened and placed very close the left chest with a fairly loosed $100 \%$ linen check shirt and a white $100 \%$ cotton singlet inner wear. The thickness of both the linen shirt and cotton inner singlet are $1 \mathrm{~mm}$ each. The stitched transmission line was placed at $5 \mathrm{~mm}$ from the body. A comparison of the On-body measurement of the stitched transmission line as well in free space was compared and the results obtained are as shown in Fig 5.27.

The human body can be thought as a relatively good conductor as it has the ability to conduct electricity, having made up approximately $80 \%$ salt water. However, the human skin which forms part of the human body is different. The human skin is a lossy material and resistant to current flow, with the skin accounting for about $99 \%$ of the human body's resistance to electric current flow [5.9]. The resistivity and permittivity of the human skin vary dramatically from one person to another and even from a single person in different physical states. Sweat or grease can alter the insulating characteristics of the skin. Similarly, the skin and the clothes worn are expected to degrade the performance of the stitched transmission line because they act as a dielectric. The closer the stitched transmission line is to the human body the lossy it will be; hence spacing between the stitched transmission and the human body has to be appreciated.

The on-body transmission characteristics are seen to be better compared to the off-body though some ripples were also observed on both the measured reflection and transmission coefficients for the on body measurements; this could be attributed to the presence of the clothing worn and the distance of the transmission line to the human skin. While the ripples observed are mainly as a result of multiple reflections along the line giving rise to insertion loss ripples. Overall the on body measurements were seen to less lossy compared to the off body measured results as can be seen in Fig.2.28. A much better on-body result is quite good for wearable applications. 


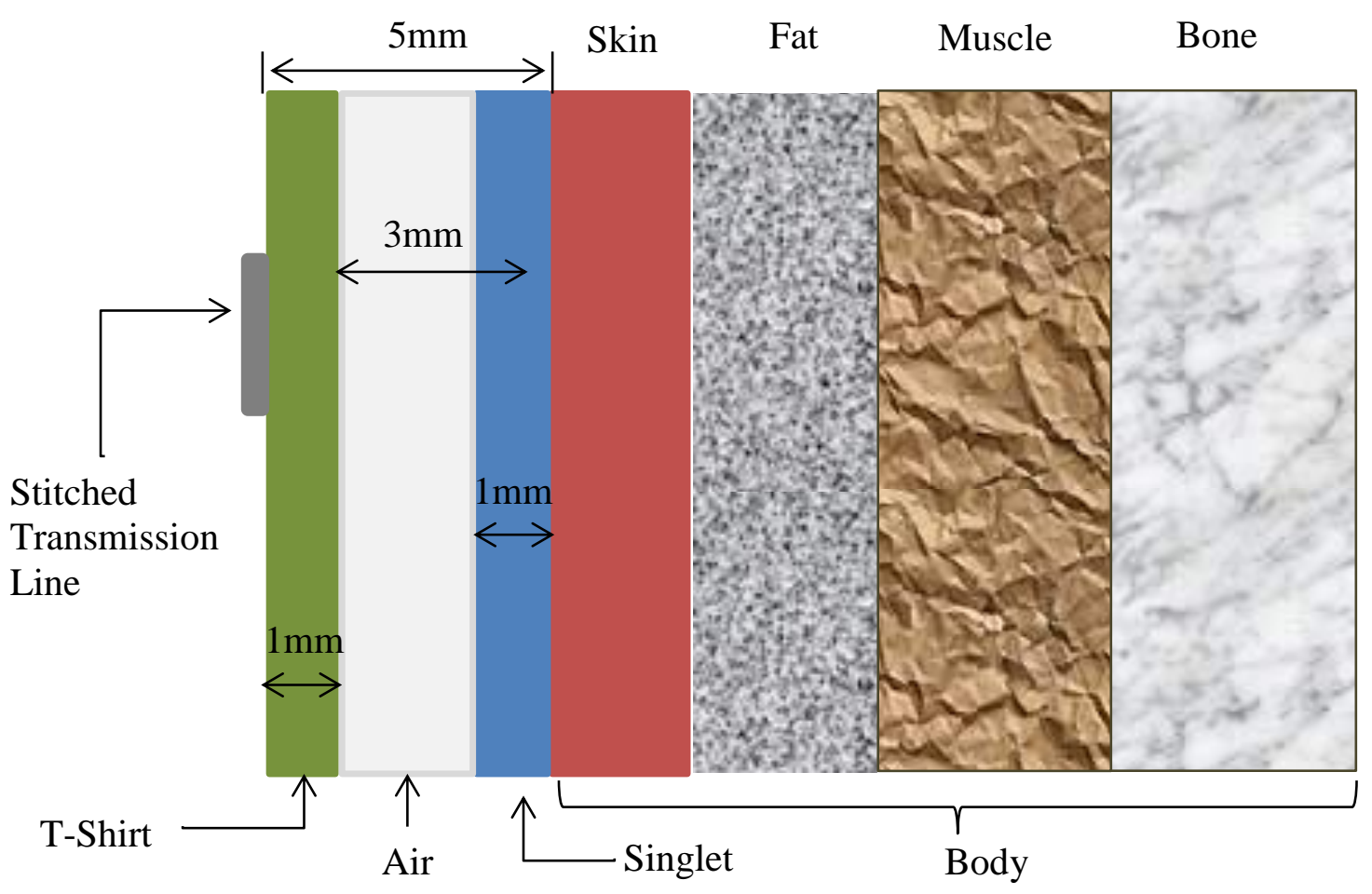

Figure 5.25 Schematic diagram of On-body measurement on the stitched transmission line depicting its proximity to the human body

TABLE 5.3 Properties of the human tissues [5.10]

\begin{tabular}{ccccc}
\hline Tissue & Permittivity $\left(\boldsymbol{\varepsilon}_{\boldsymbol{r}}\right)$ & Conductivity $\left(\boldsymbol{S m}^{\mathbf{- 1}}\right)$ & Loss Tangent & Density $\left(\mathbf{K g m}^{\mathbf{3}}\right)$ \\
\hline Skin & 31.29 & 5.0138 & 0.2835 & 1100 \\
Fat & 5.28 & 0.1 & 0.193982 & 1100 \\
Muscle & 52.79 & 1.705 & 0.24191 & 1060 \\
Bone & 12.661 & 3.8591 & 0.25244 & 1850 \\
\hline
\end{tabular}




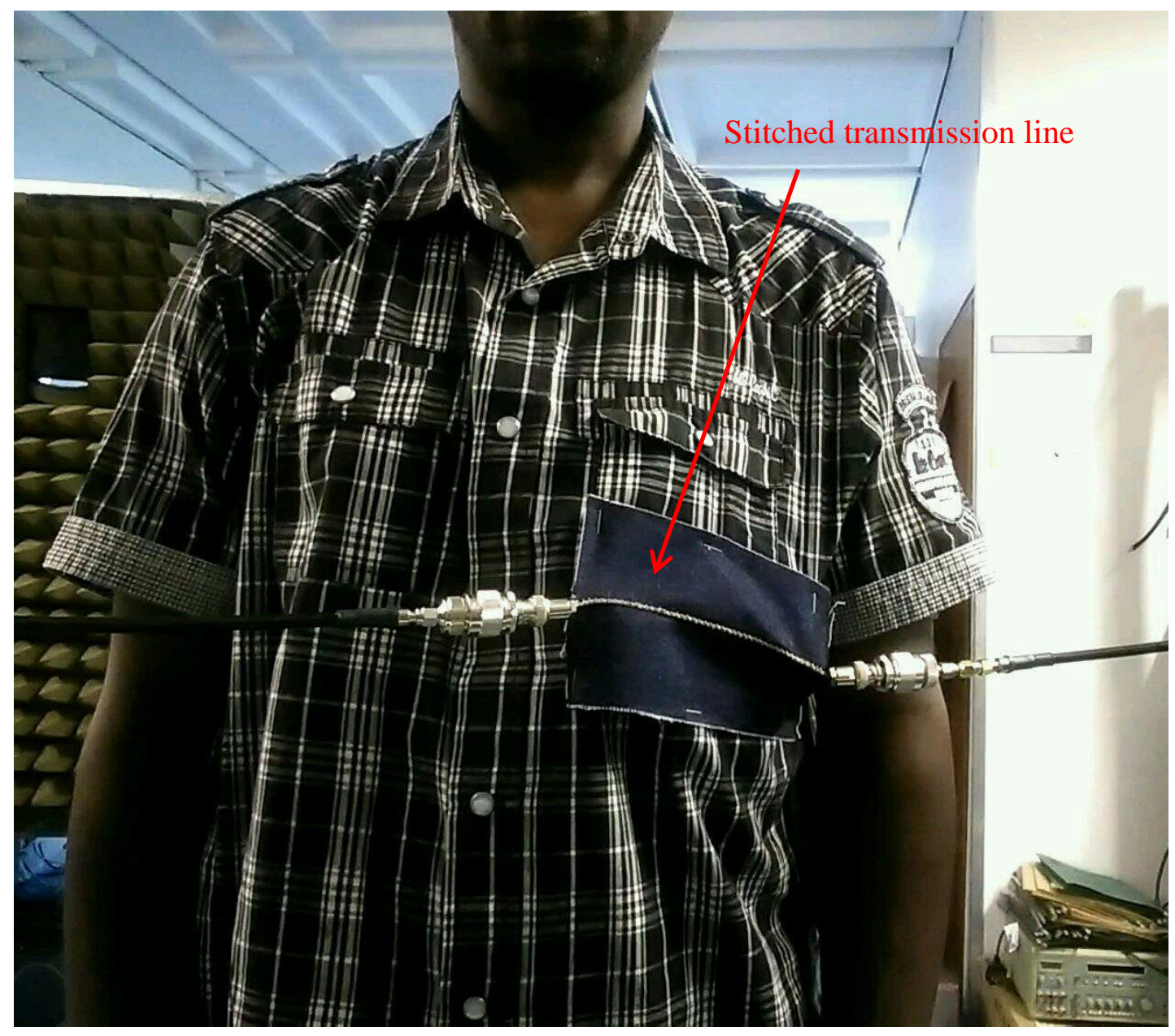

Figure 5.26 On-body measurements on the stitched transmission line 

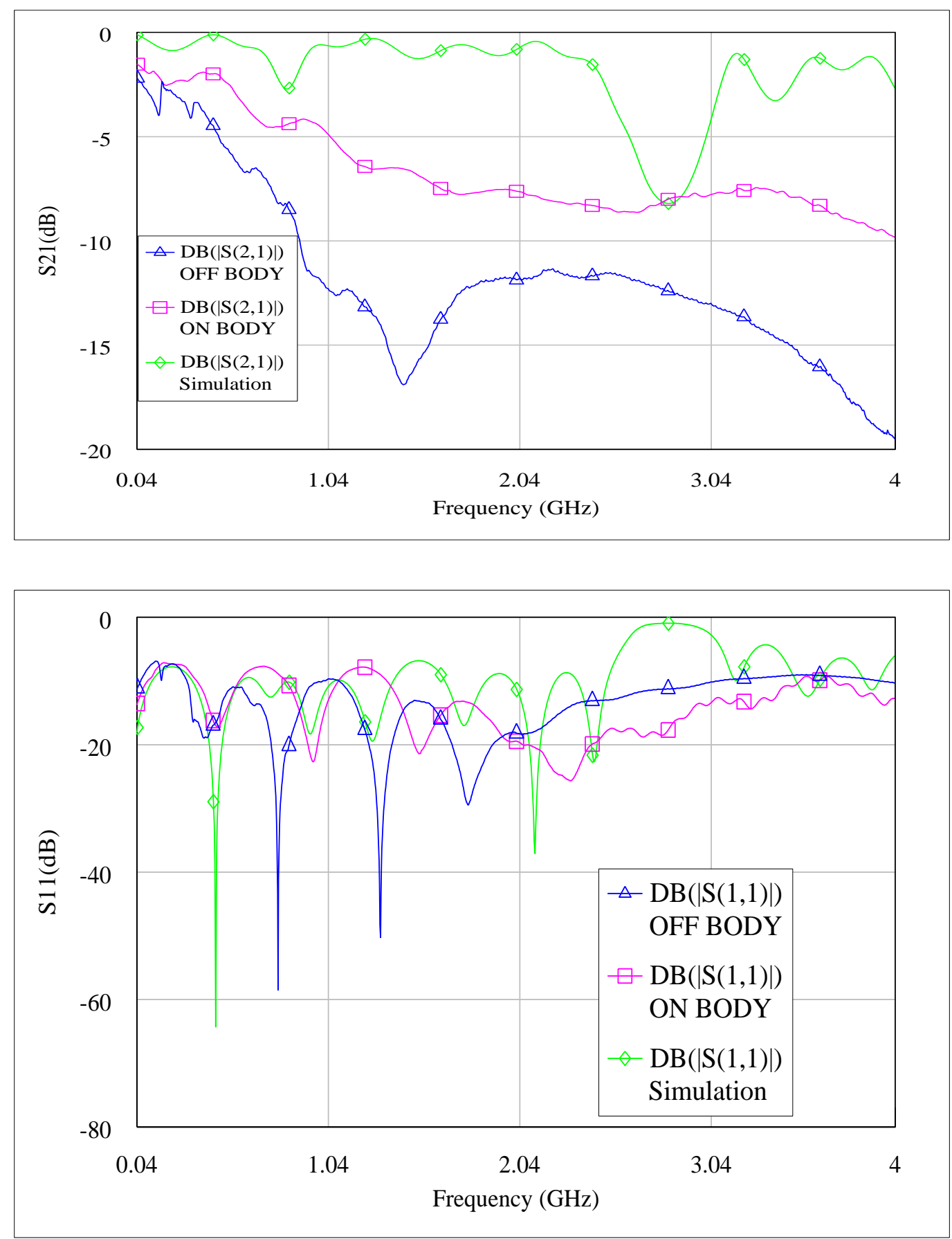

Figure 5.27 Off and On body measurements on the stitched transmission line 


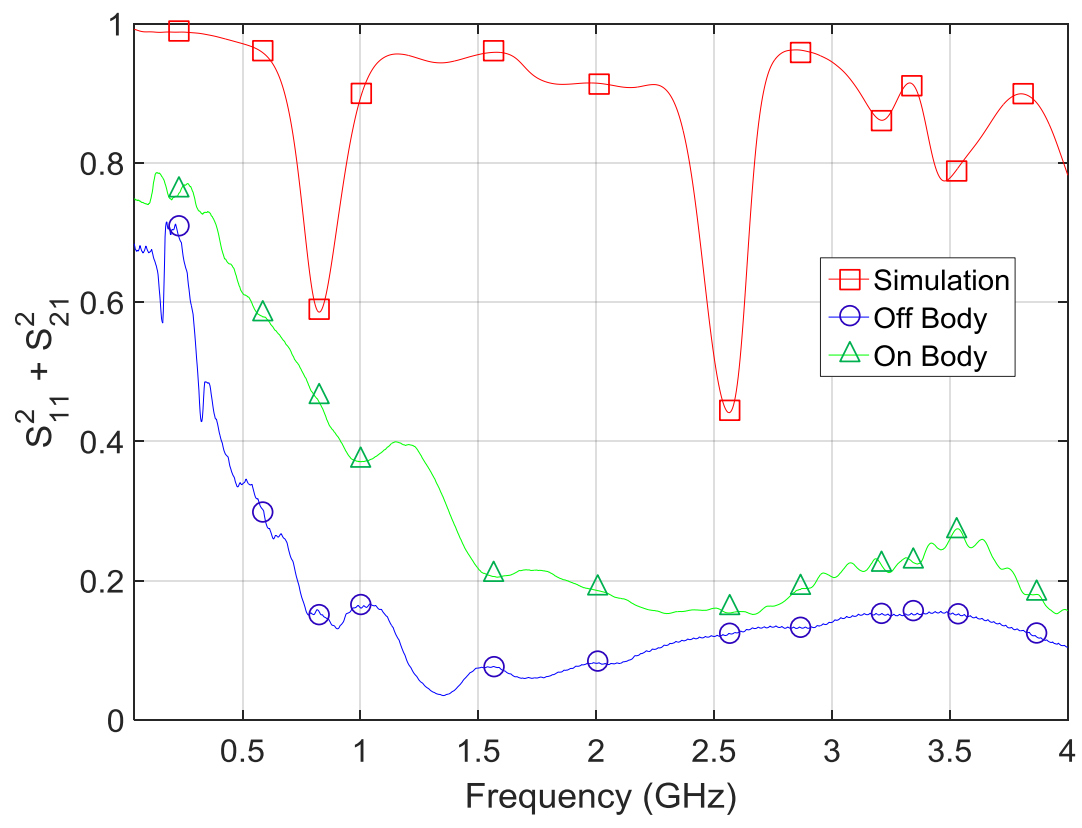

Figure 5.28 Plot of $\boldsymbol{S}_{\mathbf{1 1}}^{2}+\boldsymbol{S}_{\mathbf{2 1}}^{2}$ against frequency for off and On body measurements on the stitched transmission line

\subsection{Design Sensitivity to Manufacturing Tolerance}

With the focus on production of the stitched wearable transmission line for large-scale use or sale, the robustness of the design is been considered for deficiencies. Three features are been investigated in the subsequent subsections using CST Studio Suite and measurements using an Anritsu MS46524A 7GHz Vector Network Analyser; the transmission characteristics with two different textile substrates Denim and Felt, the sensitivity of the characteristic impedance with changes in cross sectional dimensions and the repeatability of the stitched transmission line with three different stitch angles. The dielectric properties of fabrics were first considered.

\subsubsection{Dielectric properties of fabrics}

With the integration of smart fabrics into normal fabrics, it is imperative to understand the dielectric properties of these normal fabrics. The properties of these fabrics are determined by 
the properties of the component fibers and the structure of the yarn and or the fabric. They are permeable materials and have countless small air cavities, in which the density, air volume, and size of the apertures determine its general behavior, e.g. its air permeability and thermal insulation. Accordingly, fabrics are flexible and compressible materials whose thickness and density might change with low pressures. Likewise, the main orientation of the fibers and/or yarns introduces an intrinsic planar anisotropy of general properties. Also, fibers are continuously exchanging water molecules with the surroundings, which affect their morphology and properties.

The foremost parameter of dielectrics is the permittivity, $\varepsilon$, a complex valued parameter which generally depends on frequency and temperature. It is often expressed as a relative value, $\varepsilon_{r}$, with, $\varepsilon$, given by:

$\varepsilon=\varepsilon_{0} \varepsilon_{r}$

$\varepsilon=\varepsilon_{0}\left(\varepsilon_{r}^{\mid}-j \varepsilon_{r}^{\|}\right)$

In general, the dielectric properties depend on the frequency, temperature, and surface roughness, and also on the moisture content, purity and homogeneity of the material. The real part of the relative permittivity, $\varepsilon_{r}^{l}$, is called the dielectric constant (often, $D_{k}$ ), which is not constant in frequency. The ratio of the imaginary part to real part is called the loss tangent, $\delta$, (often called material dissipation factor, $D_{f}$ ) given by:

$\tan \delta=\frac{\varepsilon_{r}^{\|}}{\varepsilon_{r}^{1}}$

\section{[5.11]}

Several researchers have studied and measured permittivity and loss tangents of nonconductive fabrics. However, with the difference in manufacturing techniques and specifications such as weight, density, and dying process, some fabrics share the same name but have different dielectric properties, while some are made from the same raw materials but also have different properties. Table 5.4 presents some measured results of some common fabrics. 
TABLE 5.4 Summary of dielectric properties of nonconductive fabrics [5.12]

\begin{tabular}{ccccc}
\hline Material & Permittivity $\left(\boldsymbol{\varepsilon}_{\boldsymbol{r}}\right)$ & $\begin{array}{c}\text { Loss } \\
\text { Tangent }(\tan \boldsymbol{\delta})\end{array}$ & Frequency $(\mathbf{G H z})$ & Reference \\
\hline Denim & $1.40-2.00$ & $0.0140-0.0700$ & $0.9,1.8,2.4,3-12,5$, & {$[5.13]-[5.18]$} \\
& & $5.2 \& 14-40$ & {$[5.19]-[5.20]$} \\
Cotton & $1.54,1.60$ & $0.0580,0.0040$ & 2.4 & {$[5.19],[5.21]$} \\
Felt & $1.36,1.38$ & $0.0160,0.0230$ & $2.4,5$ & {$[5.22],[5.19]$} \\
Leather & $1.80-2.95$ & $0.0490-0.1600$ & 2.4 & {$[5.23]-[5.24]$} \\
Velcro & 1.34 & 0.0060 & $2.7-6$ & {$[5.25]$} \\
Denim $($ Black) & 1.8 & 0.07 & $3.3 \& 5$ & {$[5.21],[5.19]$} \\
\hline Silk & $1.20,1.75$ & $0.0540,0.0120$ & 2.4 & \\
\hline
\end{tabular}

\subsubsection{Stitched Transmission Line with Different Substrates}

Two stitched transmission lines with stitch length $2 \mathrm{~mm}$ were fabricated with two different substrates Denim and Felt with relative permittivity of $\varepsilon_{r}=1.6$, loss tangent 0.05 and relative permittivity $\varepsilon_{r}=1.38$, loss tangent 0.023 respectively (see Fig. 5.29). The measured scattering parameters are as shown in Fig. 5.30, with results indicating both materials maintaining reflection coefficients $S_{11}$ below $-10 \mathrm{~dB}$. The corresponding transmission coefficients $S_{21}$ are better than $-12 d B$ for Denim and $-10 d B$ for Felt. Results obtained indicated that the losses with the Denim material are a bit more compared with the Felt which has a smaller loss tangent compared to the Denim. These losses can be seen to be quite insignificant especially at lower frequencies. From these result it is best to use Felt material as a substrate for the stitched transmission. It is also soft and has a smooth surface compared to the Denim material and well suited for wearable applications. However, some difficulties were experienced when using Felt as the stitch gets easily stuck and the Felt material gets puckered when stitching the stitched transmission line. Thus, the Denim material was preferred to the denim in constructing the stitched transmission line. 


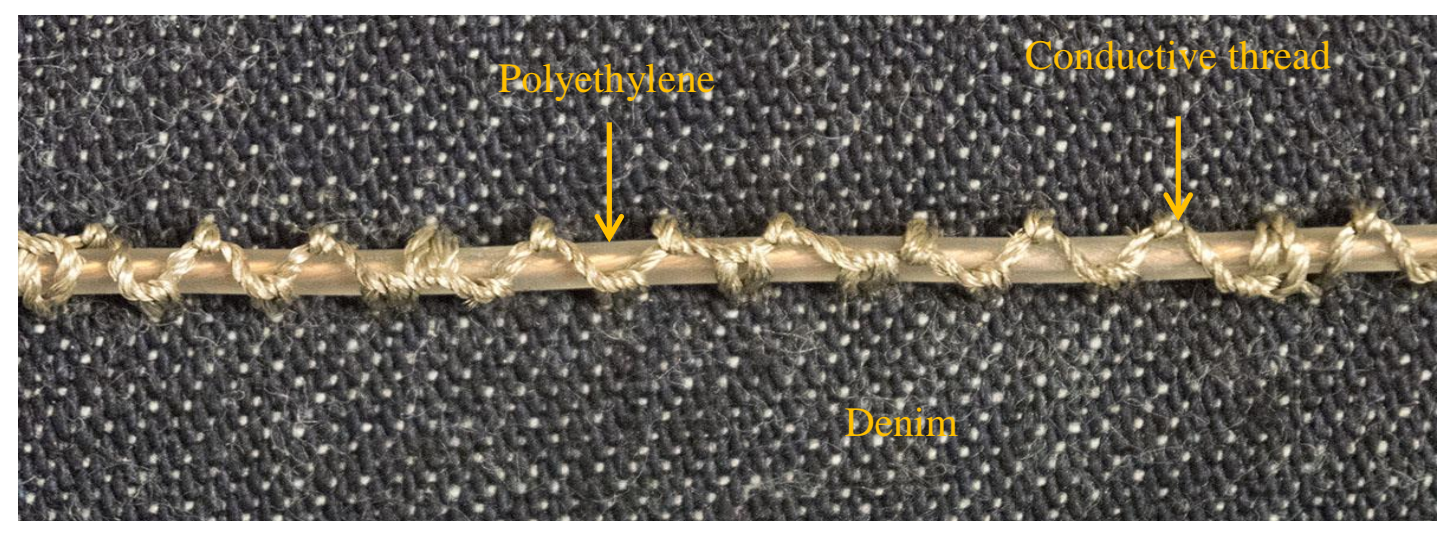

(a)

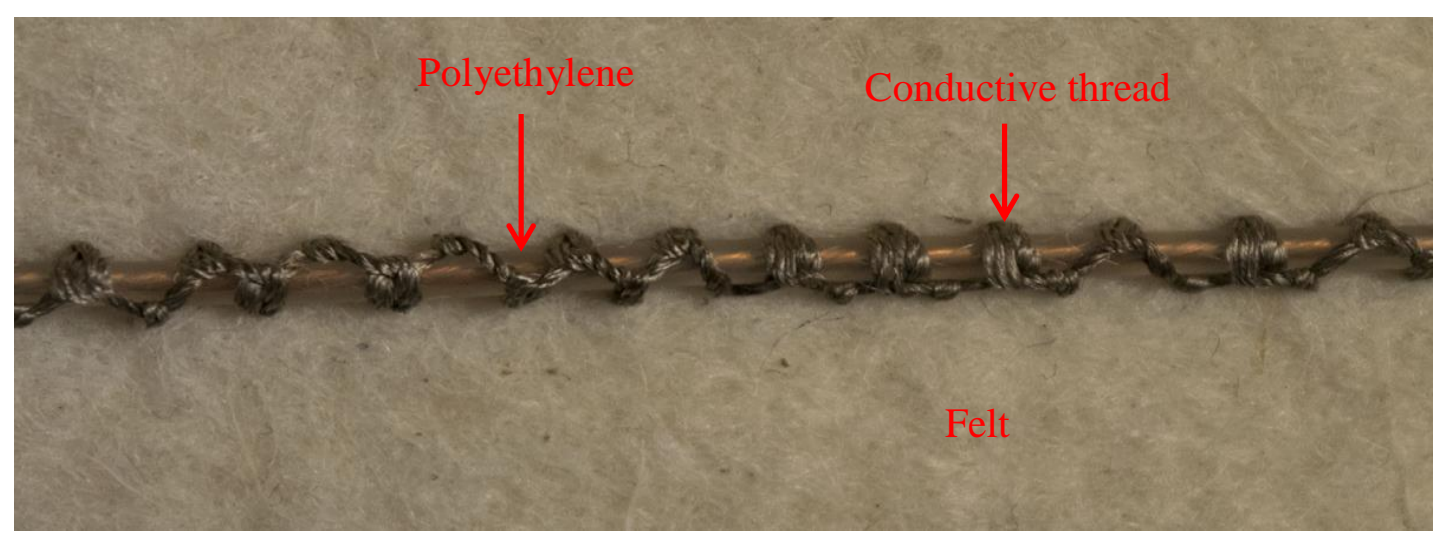

(b)

Figure 5.29 Stitched transmission line with Denim and Felt used as substrates 

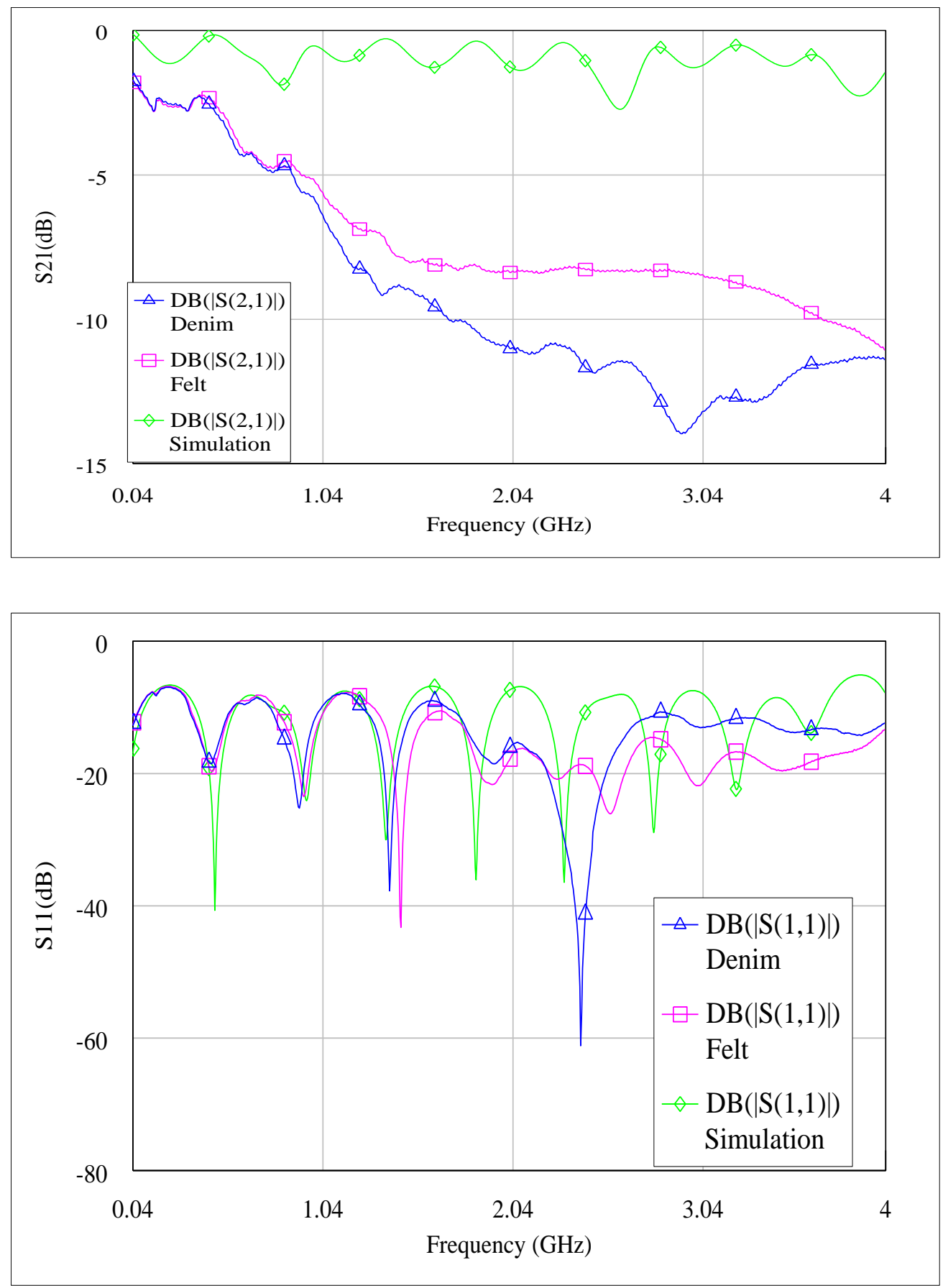

Figure 5.30 Comparison of S-Parameters between Denim and Felt used as substrates 


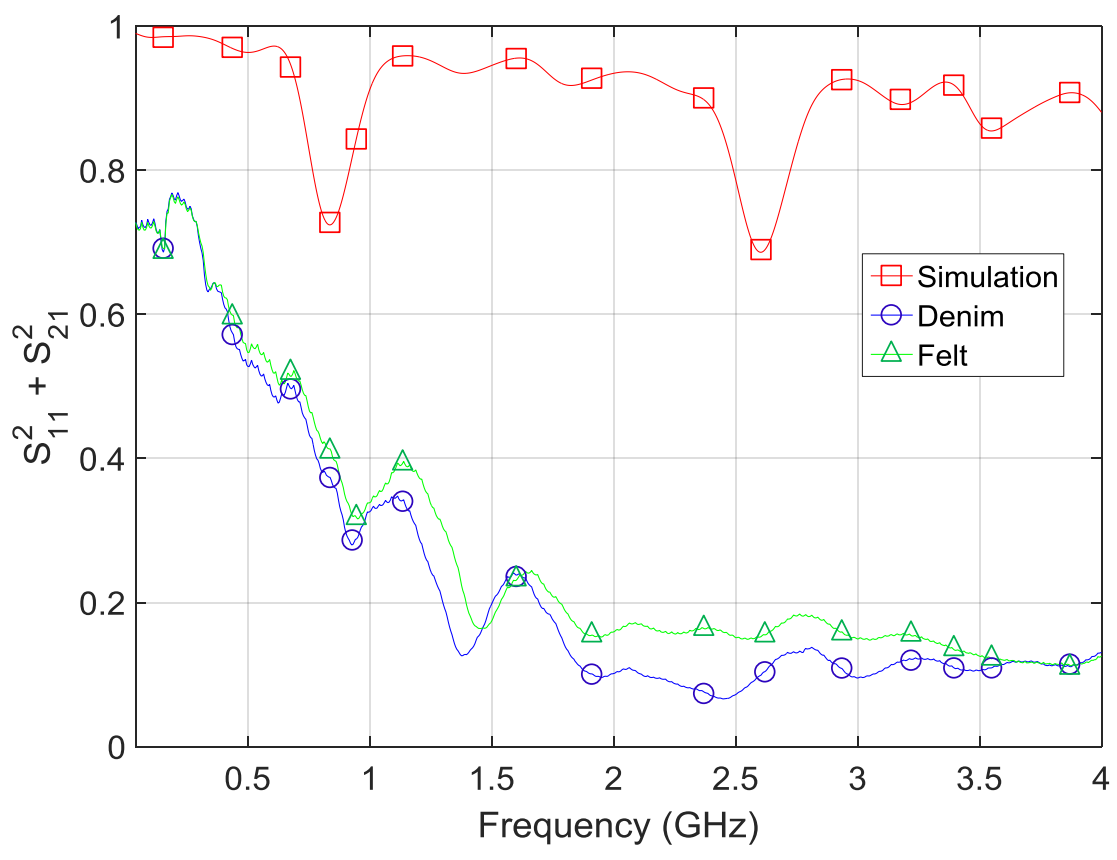

Figure 5.31 Plot of $\boldsymbol{S}_{\mathbf{1 1}}^{\mathbf{2}}+\boldsymbol{S}_{\mathbf{2 1}}^{\mathbf{2}}$ against frequency with Denim and Felt as substrates

\subsubsection{Sensitivity of the characteristic impedance with changes in cross- sectional dimensions of the stitched transmission line}

The sensitivity of the characteristic impedance of the stitched transmission line was investigated using CST Microwave Studio Suite®. Scattering parameters are always normed to reference impedance. The CST Microwave Studio Suite ${ }^{\circledR}$ provides the options of selecting the simulation process to norm the results to the calculated impedance of the port or specify the number of one's choice. It was observed from simulated results that the impedance of the stitched transmission line is sensitive to variations in cross-sectional dimensions when the outside diameter of the inner conductor, as well as the inside diameter of the stitched shield, are decreased or increased by $0.2 \mathrm{~mm}$ as can be seen in Table 5.5. Hence strict adherence to the dimensions of the stitched transmission line is necessary to maintain relatively constant impedance. 
TABLE 5.5 Simulated Characteristic Impedance of the Stitched Transmission Line with Different Cross Section Dimensions

\begin{tabular}{ccc}
\hline $\begin{array}{c}\text { Outside Diameter of Inner } \\
\text { Conductor }(\mathbf{m m})\end{array}$ & $\begin{array}{c}\text { Inside Diameter of the Shield } \\
(\mathbf{m m})\end{array}$ & $\begin{array}{c}\text { Referenced Characteristic } \\
\text { Impedance }\end{array}$ \\
& & $\mathbf{( \Omega )}$ \\
\hline 0.08 & 1.12 & 101.72 \\
0.48 & 1.52 & 45.52 \\
0.88 & 1.92 & 30.84 \\
\hline
\end{tabular}

\subsubsection{Repeatability of Stitched Transmission Line}

Repeatability otherwise known as precision or Test-retest reliability refers to the discrepancies in repeated measurements carried out under identical conditions. In order to test the reliability of the results obtained, a repeatability measurement analysis was carried out to find out the variations and irregularities in the three stitched transmission lines. Measurements were carried out on the stitched transmission line with three different stitched angles $85^{\circ}, 65^{\circ}$ and $31^{\circ}$. In each case, two stitched transmission lines were used with measurements taken within a day interval.

From Fig. 5.32, 5.33 and 5.34, it can be seen that the reflection coefficient $S_{11}$ results are consistent up to $2.5 \mathrm{GHz}$, with slight deviations beyond $2.5 \mathrm{GHz}$ which are as a result of multiple reflections along the line resulting in loss ripples, while the $S_{21}$ shows an increase loss with frequency from $0.6 \mathrm{GHz}$. Environmental conditions like temperature variations can result in thermal expansion of the cables, which can also affect measurement results. However this can be reduced by carrying out frequent calibrations as ambient temperature changes. Similarly, connector repeatability errors caused by fluctuations in the electrical characteristics of the connectors due to wear can be reduced by handling connectors with care. 

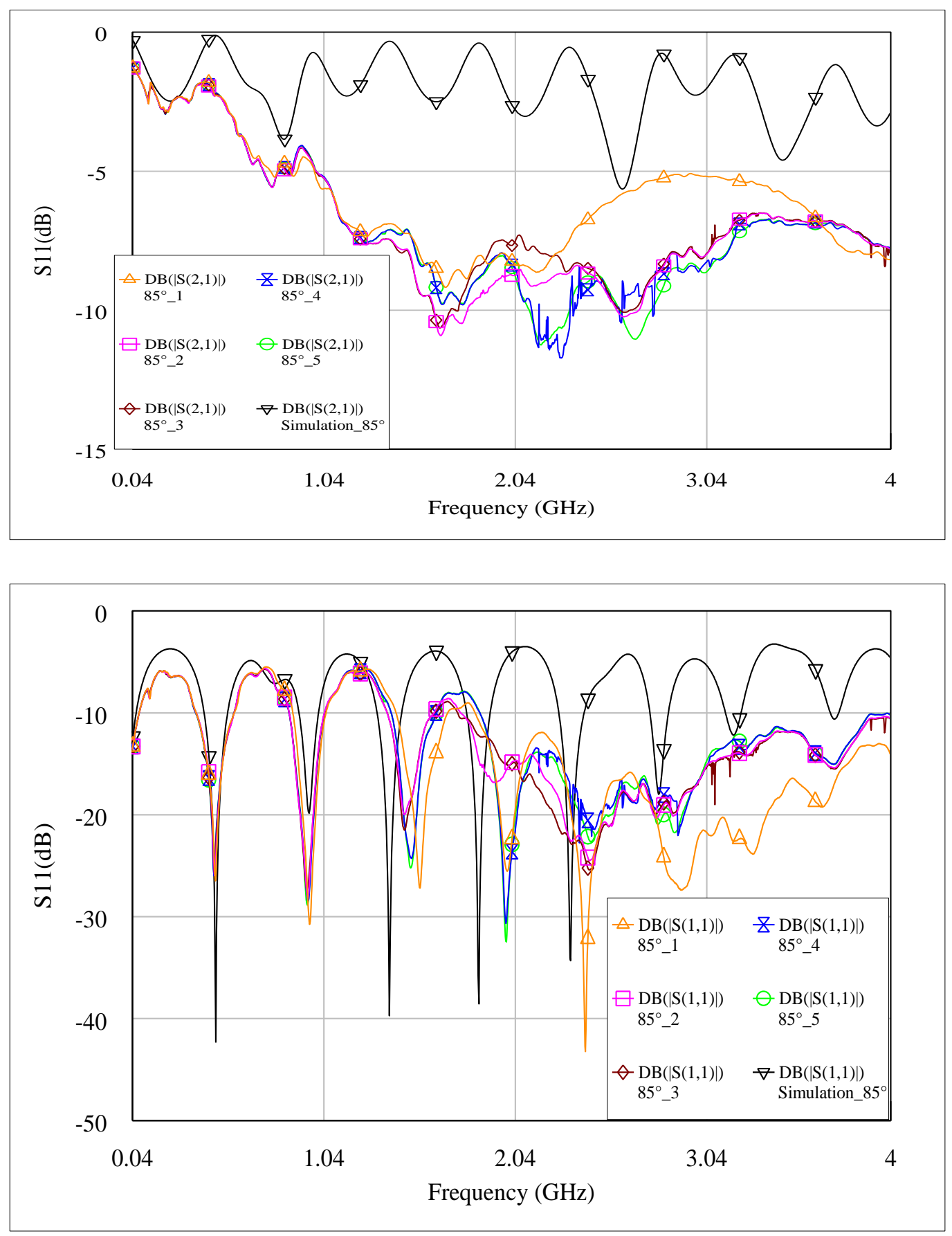

Figure 5.32 Measured S-Parameters of stitched transmission line with stitch angle $85^{\circ}$ (stitch length, $L=1.7 \mathrm{~mm}$ ) 

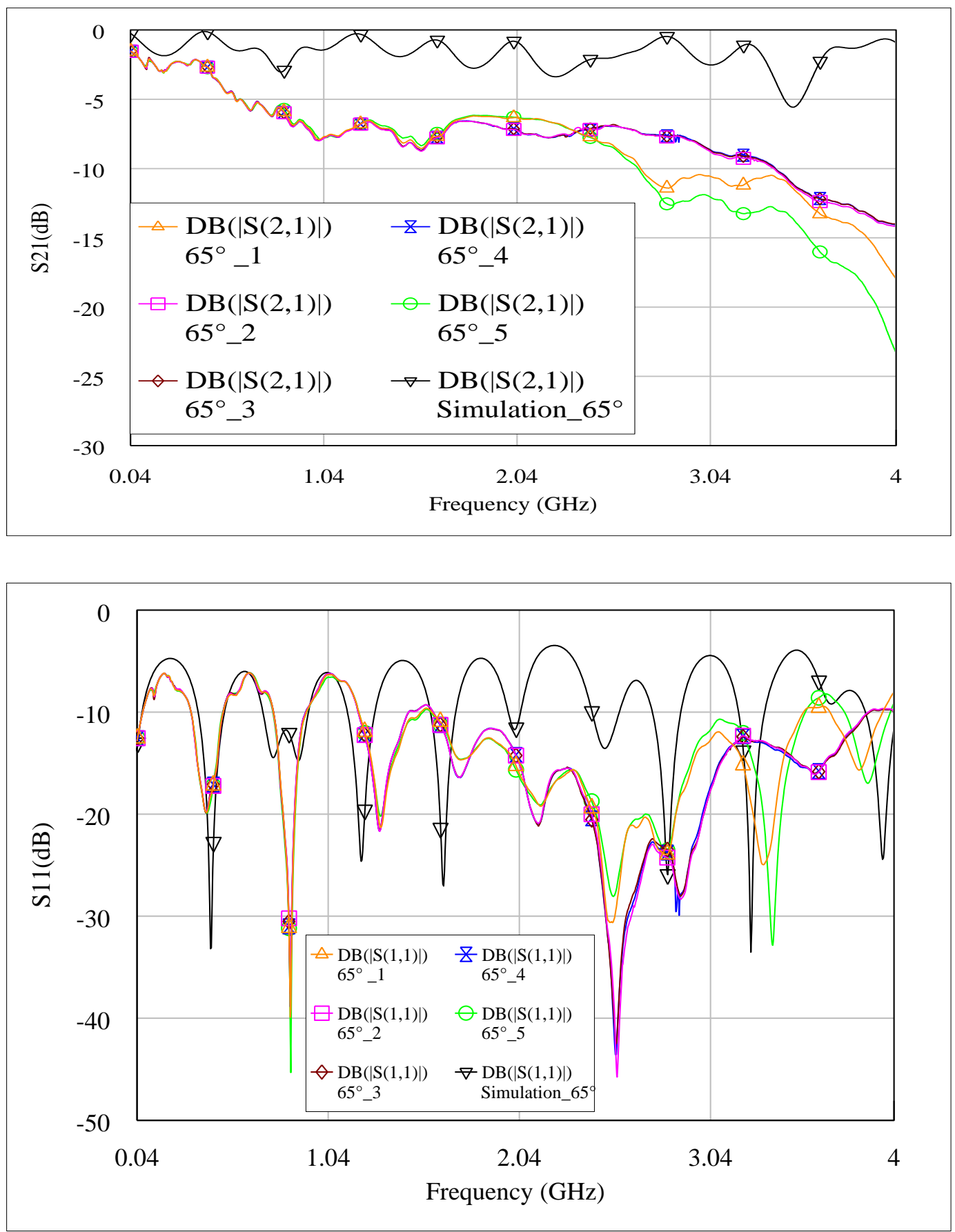

Figure 5.33 Measured S-Parameters of stitched transmission line with stitch angle $65^{\circ}($ stitch length, $L=1.5 \mathrm{~mm})$ 

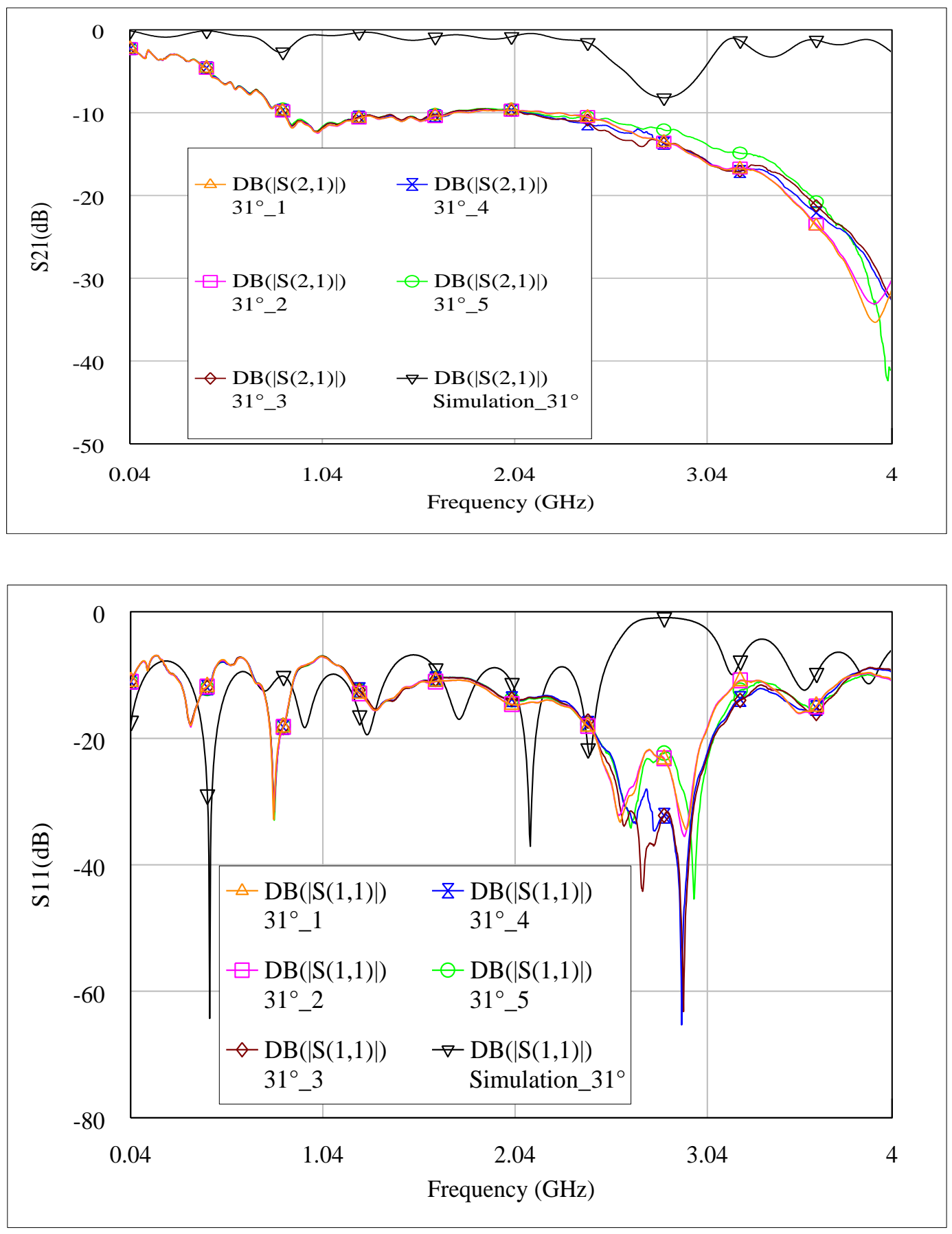

Figure 5.34 Measured S-Parameters of stitched transmission line with stitch angle $31^{\circ}($ stitch length, $L=1.2 \mathrm{~mm})$ 


\subsection{Shielding Effectiveness of the Stitched Transmission Line}

The shielding effectiveness of a braided coaxial cable can be characterized by the transfer impedance of the braid which is governed by the geometry and the materials of the shield. The transfer impedance can be computed for a range of frequencies by appropriate analytical models or advance numerical finite models. These models have been presented by Vance [5.26], Tyni [5.27] and Kley [5.28] and discussed in Chapter 3 of this thesis. We recall that the transfer impedance, $Z_{T}$, is made up of two parts the first representing the diffusion of electromagnetic energy through the stitch $Z_{D}$ and the second, $j \omega M$, representing the leakage of magnetic fields through the stitch. The diffusion component, $Z_{D}$, of the stitch is governed by DC resistance of the stitch and diffusion waves through the wall of the stitch.

$Z_{T}=Z_{D}+j \omega M$

Where

$M=M_{H}+M_{B}+M_{S}$

The inductance, $M$, in Eq. (5.4) is a superposition of the hole inductance, $M_{H}$, stitch inductance, $M_{B}$, and the skin inductance, $M_{S}$. At low frequencies, $Z_{T}$, is dominated by a wave diffusion process which is inversely proportional to the frequency(i.e. $Z_{D}$ is dominant). At high frequency, the fields' coupling through the apertures and the porpoising of the stitch takes over (i.e. the hole inductance, $M_{H}$, and stitch inductance, $M_{B}$ are dominant) while the skin inductance only yields a small contribution in the mid region (i.e. between low and high frequencies).

In the same way, the shielding effectiveness of the stitch transmission line can be determined both at low $(<1 \mathrm{GHz})$ and high frequencies. Here, the shielding effectiveness of the stitched transmission line is determined at low frequencies only $(<1 \mathrm{GHz})$. The shielding effectiveness of the stitched transmission line at low frequencies is investigated by computing the DC resistance of the shield as well as the skin depth and neglecting the leakage of the magnetic fields through the stitch.

\subsubsection{Resistance of Stitched Transmission Line}

The resistance in any electrical system is by no means constant as it depends on factors like temperature, humidity, the length of wire and high-frequency noise; hence a portion of the 
electrical energy in an electrical system is always converted to heat energy. This decreases the efficiency of the system and also causes a lot of discomfort to the user.

The zig-zag stitch was used in fabricating the stitched transmission line, where three different stitch lengths of $1.23 \mathrm{~mm}, 1.49 \mathrm{~mm}$ and $1.69 \mathrm{~mm}$ were used corresponding to three stitch angles of $31^{\circ}, 65^{\circ}$ and $85^{\circ}$ respectively.

We recall that Vance [5.26] proposed that the current flow in a braided coaxial cable is similar to that of a perforated tubular shield in which the current flows longitudinally over the shield of the coaxial cable, with the equivalent tubular DC resistance per unit length and transfer impedance, for metallic braided shield given by

$R_{D C}=\frac{4}{\pi d_{w}^{2} N_{w} C_{c} \sigma \cos \alpha}$

$Z_{T}=Z_{D}+j \omega M_{h}$

However, Tyni [5.27] and Kley [5.28] held that the current flow in a braided coaxial cable is not parallel to the cable axis but rather follows the wires in a helix-like manner. Tyni in [5.27] built on Vance's model [5.26] by introducing a third term in the transfer impedance, given by $Z_{T}=Z_{D}+j \omega\left(M_{b}+M_{h}\right)$

Similarly, in [5.28] Kley introduced a third term the skin inductance, which is due to Eddy's current in the walls of the rhombic apertures, given by

$Z_{T}=Z_{R}+j \omega\left(M_{H}+M_{B}+M_{S}\right)$

Furthermore, both Tyni and Kley tend to differ on the braid inductance $M_{b}$, as Tyni suggested that $M_{b}$ decreases by

$m_{T}^{\alpha}=\left(\frac{\widehat{h}}{d}\right)\left(1-\tan ^{2} \alpha\right)$

While Kley assumes a decrease by a factor

$m_{K}^{\propto}=\frac{0.23}{F \cos \alpha} \cos \left(2 k_{1} \propto\right)$

In the same way, we propose that the current flow in the stitched transmission line also follows the zig-zag trace in a porpoise manner. However, it is also important to also note that crossovers can be achieved at different stitch points as the current flows on the shield of the stitched transmission line.

The equivalent model of the stitch transmission line for $N=1,2,3 \ldots M$, where $\mathrm{N}$ represents the number of stitches is shown in Fig.5.35, 5.36, 5.37 and 5.38.

For stitch angles of $85^{\circ}, 65^{\circ}$ and $31^{\circ}, R^{\prime}=0.5,0.4$ and $0.25 \Omega$ while $R=0.1 \Omega$ 


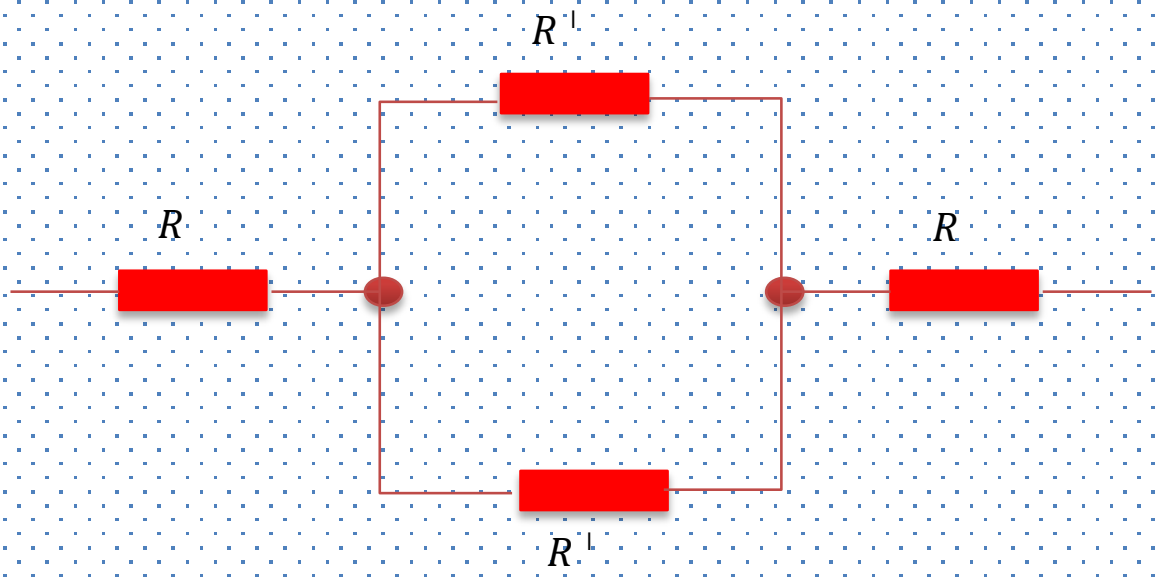

Figure 5.35 Equivalent electrical model of the stitched transmission line $(N=1)$

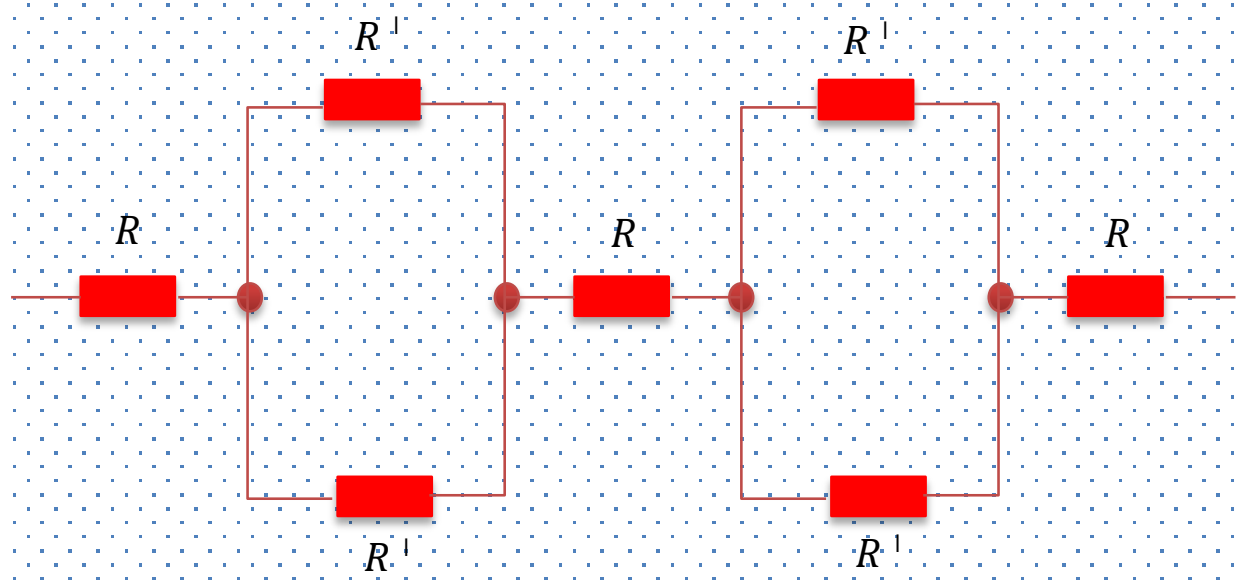

Figure 5.36 Equivalent electrical model of the stitched transmission line $(N=2)$ 


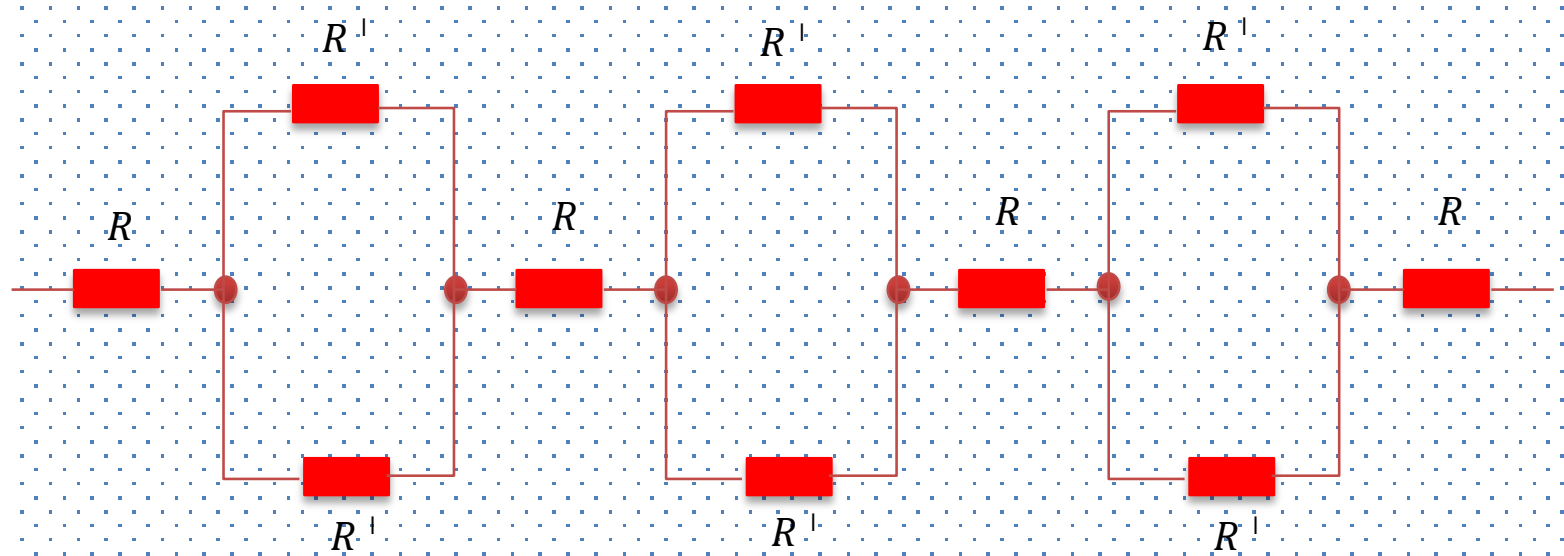

Figure 5.37 Equivalent electrical model of the stitched transmission line $(N=3)$

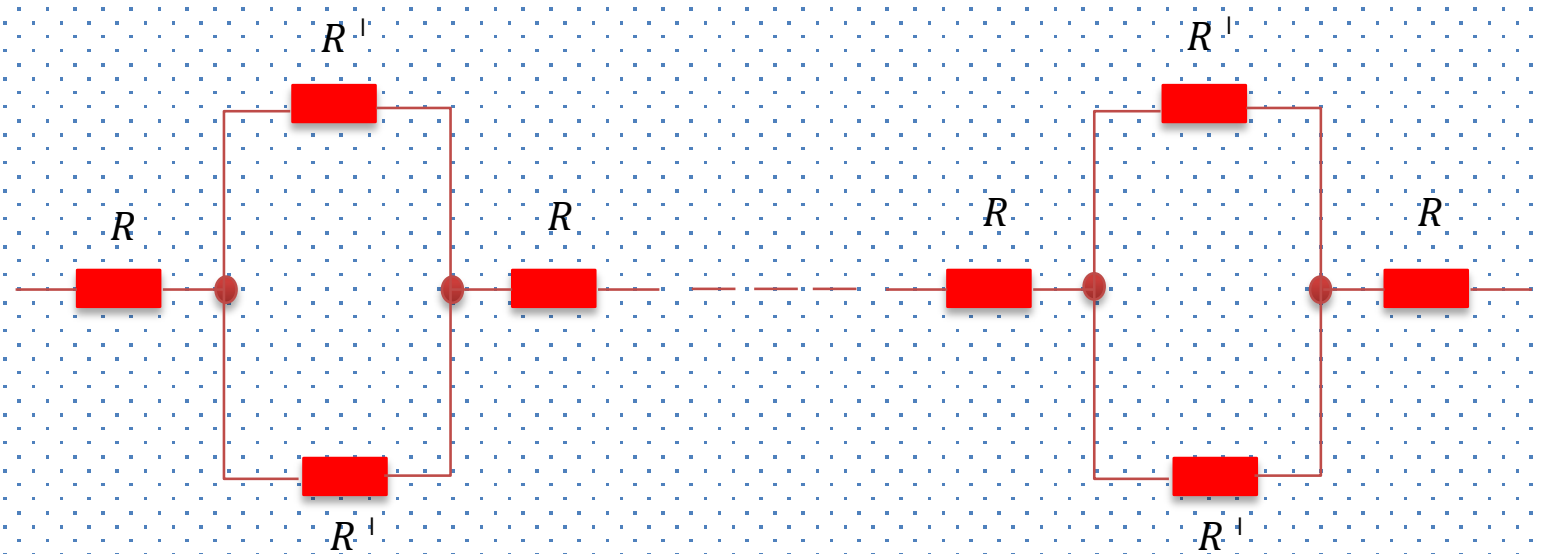

Figure 5.38 Equivalent electrical model of the stitched transmission line $(N=M)$ 
For $N=1$

$R_{1}=2 R+\frac{R^{1}}{2}$

For $N=2$

$R_{1}=3 R+R^{\prime}$

For $N=3$

$R_{1}=4 R+\frac{3}{2} R^{\prime}$

For $N=M$

$R_{M}=[(M+1) R]+\frac{M}{2} R^{\prime}$

TABLE 5.6 DC resistance of the shield of stitched transmission line

\begin{tabular}{|c|c|c|c|c|c|c|c|c|}
\hline $\begin{array}{c}\text { Conductive } \\
\text { Thread }\end{array}$ & $\begin{array}{c}\text { Resistivity } \\
\text { of coated } \\
\text { material } \\
(\Omega . m m)\end{array}$ & $\begin{array}{c}\text { DC } \\
\text { resistance } \\
\text { of } \\
\text { conductive } \\
\text { thread } \\
(\Omega / \mathbf{m m})\end{array}$ & $\begin{array}{c}\text { Stitch } \\
\text { Angle } \\
\left(^{\circ}\right)\end{array}$ & $\begin{array}{l}\text { Number } \\
\text { of stitches }\end{array}$ & $\begin{array}{l}\text { Pitch } \\
(\mathrm{mm})\end{array}$ & $\begin{array}{l}\text { Stitch } \\
\text { Length } \\
(\mathrm{mm})\end{array}$ & $\begin{array}{c}\text { Computed DC } \\
\text { resistance of } \\
\text { the shield of } \\
\text { the stitched } \\
\text { transmission } \\
\text { line }(\Omega)\end{array}$ & $\begin{array}{l}\text { Measured DC } \\
\text { resistance of the } \\
\text { shield of the } \\
\text { stitched } \\
\text { transmission line } \\
(\Omega)\end{array}$ \\
\hline Light & Silver & & 85 & 60 & 2.76 & 1.69 & 21 & 14 \\
\hline Stitches & 1.59 & 0.04 & 65 & 90 & 1.94 & 1.49 & 27 & 18 \\
\hline & $\times 10^{-8}$ & & 31 & 162 & 1.38 & 1.23 & 37 & 27 \\
\hline
\end{tabular}

Hence, it can be seen from the results obtained that the DC resistance of the shield increases with decrease stitch angle. Of course, a decrease in stitch angle results in an increase number of stitches. The divergence between the measured results and the computed results could be as a result of measurements taken with the connectors, the presence of the lead resistance, dirty connections, and temperature influence.

For a specified cable length, transfer impedance relates a current on the surface of a shield to the voltage drop generated by this current on the opposite surface of the shield. This also tells us how susceptible the inner conductor is to interference from the outside, and how much power is constrained from leaving the inside. All other things being equal, shields with lower DC resistance will have a lower voltage drop and thus lower transfer impedance. Transfer 
impedance is used to determine shield effectiveness at lower frequencies against both ingress and egress of interfering signals. Consequently, shields with lower transfer impedance are more effective than shields with higher transfer impedance.

\subsubsection{Skin effect}

In every AC carrying conductor, the magnetic field around the axis of the conductor produces variations in the current density. In an isolated conductor, the current tends to flow more densely in the outermost parts, farthest from the axis of the conductor and decreases with greater depths of the conductor. The current flows mainly at the skin of the conductor, hence the name "skin effect". This effect is caused by electromagnetic induction where a timevarying magnetic field is accompanied by a time varying induced electric field, which in turn creates a secondary time varying induced currents and a secondary magnetic field. From Lenz's law, the induced currents which produce the magnetic flux is opposite to the external flux (which "produce" the induced currents), so the total flux is reduced. Skin effect is more pronounced as the frequency increases, or as the section of the conductor becomes large. Hence, the larger the conductivity, the larger the induced currents are, the larger the permeability and the more pronounced is the flux reduction. As a result, both the total time varying magnetic field and induced currents inside conductors are reduced when compared with the DC case [5.29].

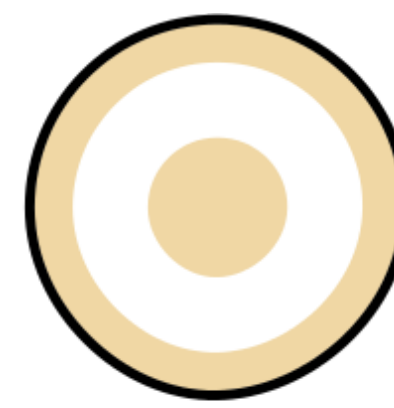

(a)

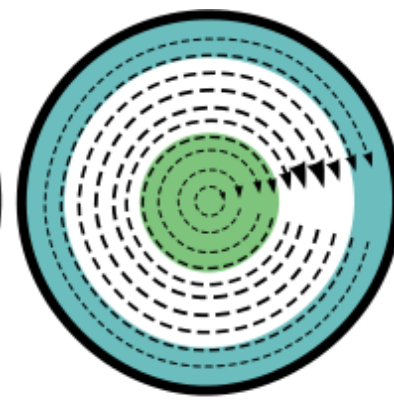

(b)

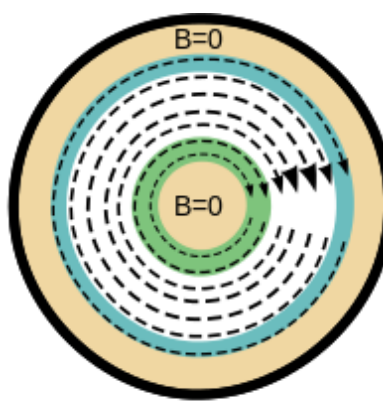

(c)

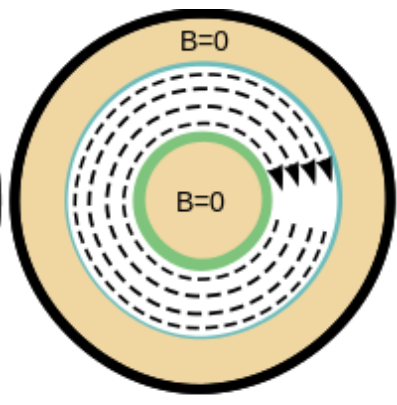

(d)

Colour code: black $=$ overall insulating sheath, $\tan =$ conductor, white $=$ dielectric, green $=$ current into the diagram, blue $=$ current coming out of the diagram, dashed black lines with arrowheads = magnetic flux $(B)$.

Figure 5.39 Cross-sectional view of a conductor with (a) non-energized, (b) low frequency, (c) middle frequency and (d) high frequency [5.30] 
TABLE 5.7 $\quad$ Skin depth $(\delta)$ for some common materials [5.31]

\begin{tabular}{ccccc}
\hline Material & $\boldsymbol{f}=\mathbf{6 0 H z}$ & $\boldsymbol{f}=\mathbf{1 0}^{\mathbf{3}} \mathbf{H z}$ & $\boldsymbol{f}=\mathbf{1 0}^{\mathbf{6}} \mathbf{H z}$ & $\boldsymbol{f}=\mathbf{1 0}^{\mathbf{9}} \mathbf{H z}$ \\
\hline Copper & $8.61 \mathrm{~mm}$ & $2.1 \mathrm{~mm}$ & $0.067 \mathrm{~mm}$ & $2.11 \mu \mathrm{m}$ \\
Iron & $0.65 \mathrm{~mm}$ & $0.16 \mathrm{~mm}$ & $5.03 \mu \mathrm{m}$ & $0.016 \mu \mathrm{m}$ \\
Seawater & $32.5 \mathrm{~m}$ & $7.96 \mathrm{~m}$ & $0.25 \mathrm{~m}$ & $7.96 \mathrm{~mm}$ \\
Wet soil & $650 \mathrm{~m}$ & $159 \mathrm{~m}$ & $5.03 \mathrm{~m}$ & $0.16 \mathrm{~m}$ \\
\hline
\end{tabular}

The AC current density $J$ in a conductor decreases exponentially from its value at the surface $J_{S}$ according to the depth $d$ from the surface, as follows:

$J=J_{S} e^{-\frac{d}{\delta}}$

Where,

$\delta=\sqrt{\frac{2 \rho}{\omega \mu}} \sqrt{\sqrt{1+(\rho \omega)^{2}+\rho \omega \varepsilon}}$

At low frequencies much below, $1 / \rho \varepsilon$, the quantity inside the radical is close to unity and the formula is more usually given as:

$\delta=\sqrt{\frac{2}{\omega \mu \sigma}}$

A measurement of the DC resistance and cross-sectional diameter of the conductive thread indicates a DC resistance of $0.43 \Omega \mathrm{cm}^{-1}\left(43 \Omega \mathrm{m}^{-1}\right.$ or $\left.13.1 \Omega \mathrm{ft}^{-1}\right)$ and a cross sectional diameter of $0.3 \mathrm{~mm}\left(0.3 \times 10^{-3} \mathrm{~m}\right)$. Thus, the resistivity can be obtained, using,

$\rho=R \frac{A}{l}$

Where $R, A$ and $l$ are the resistance, area and length of the conductive thread respectively.

The calculated resistivity of the whole metallization from (5.19) is given as $1.2 \times 10^{-5} \Omega \mathrm{m}$, while the conductivity which is given by $1 / \rho$ is $8.3 \times 10^{4} \mathrm{Sm}^{-1}$, which is quite low compared to silver with $\sigma_{s}=6.30 \times 10^{7} \mathrm{Sm}^{-1}$ [5.33] and copper with $\sigma_{c}=5.96 \times 10^{7} \mathrm{Sm}^{-1}$ [5.34] both at $20^{\circ} \mathrm{C}$ repectively. 
Using, $\quad \sigma_{c t}=8.3 \times 10^{4} \mathrm{Sm}^{-1}\left(\rho_{c t}=1.2 \times 10^{-5} \Omega m\right), \mu_{r}=1$, the skin depth of the conductive thread from Light Stitches ${ }^{\circledR}$ at $f=4 G H z$ is given by $\delta \approx 28 \mu m$. This can be seen to be quite high when compared to copper and silver with skin depth of $1.03 \mu \mathrm{m}$ and $1.00 \mu \mathrm{m}$ respectively.

\subsubsection{Transfer Impedance of the stitched Transmission Line}

The transfer impedance, $Z_{T}$, of the stitched transmission is computed for low frequency (< $1 G H z)$ is given by the diffusion term, $Z_{D}$.

$Z_{D}=\frac{R_{D C} \frac{(1+j) T}{\delta}}{\sinh \left[\frac{(1+j) T}{\delta}\right]}$

Where the DC resistance per unit length of the shield is $R_{D C}, T$, is the effective thickness of the shield and, $\delta$, is the skin depth in the shield.

Hence,

$$
Z_{D}=(0.24+j 1.09) \Omega
$$

For the stitched transmission line, the electric field contribution to the internal cable responses is relatively unimportant at low frequencies where the diffusion component of the magnetic field coupling is dominant. It was suggested, however, that at higher frequencies, the electric field coupling can be significant and, depending on the configuration and external excitation of the cable, this should be included in estimating interference effects. The diffusion term decreases rapidly with frequency, and hence the high-frequency coupling is dominated by aperture effects and increases linearly with frequency [5.35] - [5.36]. A shield with lower transfer impedance is desirable, as shields with lower transfer impedance are more effective compared with shields with higher transfer impedance.

Thus the transfer impedance, $Z_{T}$, of the stitched transmission line at low frequencies $(<$ $1 G H z)$ given by the diffusion term, $Z_{D}$, is:

$$
\begin{gathered}
Z_{T}=Z_{D} \\
Z_{T}=(0.24+j 1.09) \Omega
\end{gathered}
$$

Here, the magnitude and phase of the transfer impedance, $Z_{T}$, is given by 


$$
\left|Z_{T}\right| \cong 1.1 \Omega \Varangle 77.6^{\circ}
$$

\subsection{Conclusion}

In this chapter, measurements were carried out on the stitched transmission line with three different stitch angles and stitch types, two different substrates, two different bending angles and when subjected to some washing cycles.

The experimental results were seen to comparatively align with the CST results. The CST model was a braided coaxial cable with two annealed copper helices as the shield, which was compared to the fabricated stitched transmission line, constructed with conductive thread and copper wires and with conductive threads only as its shield.

The DC resistance of the stitched transmission line with three different stitch angles $85^{\circ}, 65^{\circ}$ and $31^{\circ}$ corresponding to the number of stitches 60,90 and 162 were computed using a DC model of the shield, and a relationship was derived for computing the DC resistance of the stitch transmission line for any given number of stitches. This was used to compute the transfer impedance of the stitched transmission line at low frequencies $(<1 G \mathrm{~Hz})$ where it is expected that the dominant factor is the diffusion component of the magnetic field coupling.

The measured results validated the conclusions drawn from the DC results with respect to the stitch angles and number of stitches, where it was also observed from measured results that the stitched transmission lines with larger stitch angles have less DC losses at lower frequencies but increased radiation losses, which are ascribed to poor shield coverage. These radiation losses increases with increase in frequencies. 


\section{REFERENCE}

5.1. RG174 [Online]. [Accessed: 12-February 2017]. Available from:

http://www.farnell.com/datasheets/1712694.pdf

5.2. Z. Xu, T. Kaufmann, C. Fumeaux, "Wearable Textile Shielded Stripline for Broadband Operation," Microwave and Wireless Components Letters, IEEE, vol.24, no.8, pp.566568, August 2014.

5.3. J. Wang and O. Fujiwara, "TDR Analysis of Electromagnetic Radiation from a Bend of Microstrip Line," IEICE Trans. Commun., vol.E88-B, NO.8, pp. 3207-3212, AUGUST 2005

5.4. J. Li, Yaojiang-Zhang, D. Liu, A. Bhobe, J. L. Drewniak and J. Fan, "Radiation Physics from Two-Wire Transmission Lines," 2015 IEEE Symposium on Electromagnetic Compatibility and Signal Integrity, Santa Clara, CA, pp. 160-164, 2015

5.5. D. S. Gorjanc and V. Bukosek, "The Behaviour of Fabric with Elastane Yarn During Stretching," FIBRES \& TEXTILES in Eastern Europe, Vol. 16, No. 3 (68), pp. 63-68, July / September 2008

5.6. D. Cottet, J. Grzyb, T. Kirstein and G. Tröster, "Electrical Characterisation of Textile Transmission Lines," IEEE Transaction on Advance Packaging, Vol. 26, N0. 2, pp. 182-190, May 2003

5.7. M. Braunovic, V. V. Koncvhits and N. K. Myshikin, "Electrical Contacts, Fundamentals, Applications and Technology," CRC Press, pp.76-77, 2006

5.8. Q150R Rotary-Pumped Sputter Coater/Carbon Coater [Online]. [Accessed 02-March 2017]. Available from: https://www.quorumtech.com/quorum-product/q150r-rotarypumped-sputter-coatercarbon-coater

5.9. R. M. Fish, L. A. Geddes, "Conduction of Electrical Current to and Through the Human Body: A Review,” Eplasty 9, e44, pp. 407-421, 2009

5.10. U. Ali, S. Ullah, J. Khan, M. Shafi, B. Kamal, A. Bashir, J. A. Flint and R. D. Seager, "Design and SAR Analysis of Wearable Antenna on Various Parts of Human Body, Using Conventional and Artificial Ground Planes," Journal of Electrical Engineering \& Technology, 12(1), pp. 317-328, 2017

5.11. J. Baker-Jarvis, M.D. Janezic, D.C. DeGroot, "High-Frequency Dielectric Measurements," IEEE Instrumentation and Measurement Magazine, 13, pp.24-31 April 2010 
5.12. S. Zhang, "Design advances of embroidered fabric antennas," Ph.D. thesis, School of Electronic, Electrical and Systems Engineering, Loughborough University, Loughborough, 2014

5.13. B. Sanz-Izquierdo and J. C. Batchelor, "A Dual Band Belt Antenna," in International Workshop on Antenna Technology: Small Antennas and Novel Metamaterials, 2008, pp. 374-377

5.14. D. Gaspar and A. A. Moreira, "Belt antenna for wearable applications," in IEEE Antennas and Propagation Society International Symposium, 2009, pp. 1-4

5.15. B. Sanz-Izquierdo, J. C. Batchelor, and M. I. Sobhy, “Compact UWB Wearable Antenna," in Loughborough Antennas and Propagation Conference (LAPC), 2007, pp. $121-124$

5.16. B. Sanz-Izquierdo, J. C. Batchelor, and M. I. Sobhy, "Button antenna on textiles for wireless local area network on body applications," IET Microwaves, Antennas \& Propagation, vol. 4, no. 11, pp. 1980-1987, 2010

5.17. S. W. Harmer, N. Rezgui, N. Bowring, Z. Luklinska, and G. Ren, "Determination of the complex permittivity of textiles and leather in the $14-40 \mathrm{GHz}$ millimetre-wave band using a free-wave transmittance only method," IET Microwaves, Antennas \& Propagation, vol. 2, no. 6, pp. 606-614, 2008

5.18. B. Sanz-Izquierdo, L. Wu, J. C. Batchelor, and P. R. Young, "Textile integrated waveguide slot antenna," in IEEE Antennas and Propagation Society International Symposium, 2010, pp. 1-4

5.19. S. Bashir, "Design and synthesis of non-uniform high impedance surface based wearable antennas," PhD thesis, School of Electronic, Electrical and Systems Engineering, Loughborough University, Loughborough, 2009

5.20. Y. Ouyang and W. J. Chappell, "High frequency properties of electro-textiles for wearable antenna applications," Antennas and Propagation, IEEE Transactions on, vol. 56, no. 2, pp. 381-389, 2008

5.21. S. Zhu and R. Langley, "Dual-band wearable textile antenna on an EBG substrate," Antennas and Propagation, IEEE Transactions on, vol. 57, no. 4, pp. 926-935, 2009

5.22. D. Gaspar and A. A. Moreira, "Belt antenna for wearable applications," in IEEE Antennas and Propagation Society International Symposium, 2009, pp.1-4 
5.23. B. Sanz-Izquierdo, J. C. Batchelor, and M. I. Sobhy, "Button antenna on textiles for wireless local area network on body applications," IET Microwaves, Antennas \& Propagation, vol. 4, no. 11, pp. 1980-1987, 2010

5.24. B. Sanz-Izquierdo, F. Huang, and J. C. Batchelor, "Small size wearable button antenna," in First European Conference on Antennas and Propagation (EuCAP), 2006, pp. 1-4

5.25. B. Sanz-Izquierdo, L. Wu, J. Batchelor, P. Young, "Textile Integrated Waveguide Slot Antenna," In Proceedings of the IEEE Antennas and Propagation Society International Symposium (APSURSI), Toronto, ON, USA, pp. 1-4, 11-17 July 2010

5.26. E. Vance, "Shielding effectiveness of braided-wire shields," IEEE Transactions on Electromagnetic Compatibility, vol. EMC-17, pp. 71-77, May 1975

5.27. M. Tyni, "The transfer impedance of coaxial cables with braided outer conductor," in Wroclaw EMC Symposium, 1976, pp. 410 - 419

5.28. Thomas Kley, “Optimized single braided cable shields," IEEE Transactions on Electromagnetic Compatibility, vol. 35, no. 1, February 1993

5.29. Skin effect [Online]. [Accessed: 12-February 2017]. Available from: http://ecee.colorado.edu/ ecen3400/Textbook/Chapter\%2020\%20\%20The\%20Skin\%20Effect.pdf

5.30. Skin effect [Online]. [Accessed: 12-February 2017]. Available from: https://en.wikipedia.org/wiki/Skin_effect

5.31. Z. Popovic and B. D. Popovic, "Introductory Electromagnetics, ”Prentice Hall, pp. 385, 2000

5.32. D. M. Pozar, “Microwave Engineering,” John Wiley \& Sons, Inc., Fourth Edition, 2012

5.33. R. A. Serway, "Principles of Physics (2nd Ed.). Fort Worth, Texas; London: Saunders College Pub. pp. 602, 1998

5.34. D. Giancoli, "Electric Currents and Resistance," Physics for Scientists and Engineers with Modern Physics (4th Ed.). Upper Saddle River, New Jersey: Prentice Hall. pp. 658, 2009

5.35. H. Shippers, J. Verpoorte and R. Otin, "Electromagnetic analysis of metal braids," Proceedings of the 10th International Symposium on Electromagnetic Compatibility (EMC Europe 2011), pp. 543-548, September 2011

5.36. H. A. Roberts, S. B. MacDonald and J Copabianco, "Electric and magnetic field coupling through a braided shield cable: Transfer admittance and transfer impedance," U.S. Army Laboratory Command Harry Diamond Laboratories Adelphi, MD 207831197, August 1986 


\section{Chapter 6}

\section{Conclusion and Future Work}

\subsection{Summary}

This research work focussed on the development of a stitched transmission line. The aim is to use the idea of a braided coaxial cable to develop a transmission line that could transmit signals within a frequency range of $0.04-4.00 \mathrm{GHz}$. The advantages of using this idea include but not limited to sufficient frequency range to support multiple channel, reduced cost, noise and crosstalk. The design and simulation of the stitched transmission line was carried out with CST Microwave Studio Suite ${ }^{\circledR}$. To fabricate the stitched transmission line, the inner conductor and the insulated layer were selected as RG 174, while the stitched shield is made up of conductive threads from Light Stiches ${ }^{\circledR}$. A modified presser foot was also fabricated to aid in stitching the transmission line using Singer Talent ${ }^{\mathrm{TM}} 3321$ onto the Denim material without upsetting the tubular insulating medium surrounding the inner conductor.

Three different designs were carried out with three different stitch angles of $85^{\circ}, 65^{\circ}$ and $31^{\circ}$ and high-frequency network analyzer measurements were achieved for frequencies up to 4GHz. The extracted S-parameters indicates decrease DC losses with an increase in stitch angle which corresponds to a decrease in number of stitches at lower frequencies, with some mismatch losses while radiation loss tends to decrease with a decrease in stitch angle which corresponds to an increase in the number of stitches.

The performance of the stitched transmission line with three different stitch types, when bent through curved angles $90^{\circ}$ and $180^{\circ}$, when placed close to the human body and when subjected to washing cycles were also investigated and results presented.

Also, with the focus on production of the stitched wearable transmission line for large-scale use or sale, the robustness of the design was considered for deficiencies. Three features were investigated using CST Microwave Studio Suite ${ }^{\circledR}$ and measurements using Anritsu MS46524A 7GHz Vector Network Analyser. These includes, the transmission characteristics with two different textile substrates Denim and Felt, the sensitivity of the characteristic impedance with changes in cross sectional dimensions and the repeatability of the stitched 
transmission line with three different stitch angles. Results obtained also indicated that the losses increased with Denim as the substrates, though these losses are mostly insignificant especially at lower frequencies, while the CST results indicated an increase in impedance of the stitched transmission line with decrease cross section dimensions and vice versa. Finally the repeatability measurement results showed consistency up to $2.5 \mathrm{GHz}$, with slight deviations above that which are as a result of multiple reflections along the line and losses in the form of radiations.

\subsection{Main Contributions}

The main contributions of this thesis are:

- Numerical modeling of the stitched transmission line within the frequency range of $0.04-4 \mathrm{GHz}$. The stitched transmission line was modelled as a braided coaxial cable with two helices. The numerical modelling with CST was conducted with six different stitch lengths $1.69 \mathrm{~mm}, 1.49 \mathrm{~mm}, 1.23 \mathrm{~mm}, 2 \mathrm{~mm}, 3 \mathrm{~mm}$ and $4 \mathrm{~mm}$. With the first three stitch lengths of $1.69 \mathrm{~mm}, 1.49 \mathrm{~mm}$ and $1.23 \mathrm{~mm}$ corresponding to stitch angles $85^{\circ}, 65^{\circ}$ and $31^{\circ}$.

- Development of a novel presser foot that will suite the requirement for use with the RG174 coaxial cable in the construction of the stitched transmission line. In the proposed technique the presser foot of the sewing machine was replaced with an improved presser foot for use solely with a stripped RG174 coaxial cable.

- Numerical analysis of the DC resistance of the three different stitch lengths $1.69 \mathrm{~mm}, 1.49 \mathrm{~mm}$ and $1.23 \mathrm{~mm}$ corresponding to stitch angles $85^{\circ}, 65^{\circ}$ and $31^{\circ}$ of the stitched transmission line was carried out. To achieve this, a DC representation of the stitched shield was proposed and a mathematical relationship was derived for computing the DC resistance of the stitch transmission line for any given number of stitches. This was subsequently used in computing the transfer impedance of the stitch transmission line at low frequencies $(<1 \mathrm{GHz})$, with results showing that the shield of stitched transmission line to be more effective at low frequencies.

- Experimental characterisation of the stitched wearable transmission line. Vector network analyzer (VNA) measurements of the stitched transmission line within the frequency range of $0.04-4 G H z$ were achieved with six different stitch 
lengths $1.69 \mathrm{~mm}, 1.49 \mathrm{~mm}, 1.23 \mathrm{~mm}, 2 \mathrm{~mm}, 3 \mathrm{~mm}$ and $4 \mathrm{~mm}$. First measurements with three different stitch lengths of $1.69 \mathrm{~mm}, 1.49 \mathrm{~mm}$ and $1.23 \mathrm{~mm}$ corresponding to stitch angles $85^{\circ}, 65^{\circ}$ and $31^{\circ}$, were carried out, followed by three different measurements with conductive threads stitched alongside copper wires stitch types for three different stitch lengths of $2 \mathrm{~mm}, 3 \mathrm{~mm}$ and $4 \mathrm{~mm}$, when bent through curved angle of $90^{\circ}$ and $180^{\circ}$, when subjected to washing cycles, when placed on the human body and lastly with two different substrates (Denim and Felt).

\subsection{Industrial Applications}

This thesis has shown the feasibility of fabricating a novel stitched transmission line with the aid of a novel presser foot using any sewing machine. The flexibility and light weight of this device makes it a good candidate for integration into apparels.

Mass scale production of the stitched transmission line is feasible as the manufacturing process is easy and reliable, and cost less especially with the preferred choice of the sewing machine in place of the embroidery machine. The effects of the stitched parameters on the stitched transmission line have been evaluated and presented which also makes it easier for the industry to evaluate the cost and performance ratio at the same time.

Finally, the stitched transmission line is not restricted to military or space technology alone as the wearability qualities and low cost will make it suitable and affordable for civilian uses.

\subsection{Future Research}

This thesis is mainly focussed on the fabrication of a stitched transmission line that could transmit signals from a frequency range $0.04-4 G H z$ using the idea of a braided coaxial cable. Based on the conclusion drawn and the limitations of the work presented, the following research areas and issues would provide potential and some progressions to the work carried out in this thesis and are worth considering.

- Integrating the stitched transmission line with antennas. Wearable transmission lines are used to transport RF signals between various pieces of communication equipment and to connect antennas to transmitters and receivers. The stitched transmission line 
was designed to meet this requirement and it is important to see how well suited it is for this purpose when integrated with an antenna.

- With the numerous apertures ascribed to the shield of the stitched transmission line, it can also be used as a radiating cable; this has an advantage of having the ability to carry multiple frequencies on a single line and to also function as a broadband antenna. Thus performing both as a transmission line and an antenna at the same time.

- Stitched transmission lines with different materials and conductive threads. The stitched transmission line can be developed with different conductive threads (insulated and non-insulated) and substrates. Similarly, further work on the washed line can also be carried out with different substrates, conductive threads (insulated and non-insulated), and using both hot and cold wash with different types of detergents.

- Transfer impedance at higher frequencies. The shielding analysis on the stitched transmission line was carried out at lower frequencies $(<1 \mathrm{GHz})$ where it is expected that the DC resistance and diffusion waves through the stitch are the dominant factors. However, at higher frequencies the fields coupling through the apertures and the porpoising of the stitch takes over (i.e. the hole inductance and stitch inductance are dominant). As such, there's a need to also examine the shielding effectiveness of the shield of the stitched transmission line at higher frequencies $(\geq 1 \mathrm{GHz})$.

- Finally, the lamination of the stitched transmission line is also another possibility, with researchers at Loughborough University developing the idea with antennas using the lamination machine as this could also aid in reducing signal egress and ingress that is common with a transmission line with a sparse shield. 


\section{REFERENCE}

6.1. Belden 8216 Coax - RG-174/U Type [Online]. [Accessed: 12-February 2017]. Available from: http://www.belden.com/techdatas/metric/8216.pdf

6.2. Farnell Element 14 BNC Plug [Online]. [Accessed: 12-February 2017]. Available: http://www.farnell.com/datasheets/1662634.pdf

6.3. K. Allman, J. Laurito and M. Loh, "Newton-Raphson Method-Financial Simulation Modelling in Excel: A step-by-step Guide,” John Wiley \& Sons, Inc., 2012

6.4. A. Sadr, "Taylor Series Expansion," [Online]. [Accessed: 12-February 2017]. Available from: $\quad$ http://onlinelibrary.wiley.com/doi/10.1002/9781118267967.app1/pdf 


\section{Appendix A}

\section{Coax - RG-174/U Type}

\section{Description:}

RG-174/U type, 26 AWG stranded (7x34) .019" bare copper-covered steel conductor, polyethylene insulation, tinned copper braid shield (90\% coverage), PVC jacket.

\section{Physical Characteristics (Overall)}

\section{Conductor}

AWG:

\# Coax AWG Stranding Conductor Material Dia. (mm)

$$
26 \quad 7 \times 34 \quad \text { BCCS - Bare Copper Covered Steel }
$$

Insulation

Insulation Material:

Insulation Material Dia. (mm)

PE - Polyethylene 1.524

Outer Shield

Outer Shield Material:

Type Outer Shield Material Coverage (\%)

TC - Tinned Copper

Outer Jacket

Outer Jacket Material:

Outer Jacket Material

PVC - Polyvinyl Chloride

Overall Cabling

Overall Nominal Diameter:

$2.794 \mathrm{~mm}$

\section{Mechanical Characteristics (Overall)}

Operating Temperature Range:

$-40^{\circ} \mathrm{C}$ To $+75^{\circ} \mathrm{C}$

UL Temperature Rating:

$60^{\circ} \mathrm{C}$ (UL AWM Style 1354)

Bulk Cable Weight:

$12.501 \mathrm{Kg} / \mathrm{Km}$

Max. Recommended Pulling Tension:

$93.412 \mathrm{~N}$

Min. Bend Radius (Install)/Minor Axis:

$25.400 \mathrm{~mm}$

\section{Applicable Specifications and Agency Compliance (Overall)}

Applicable Standards \& Environmental Programs

\begin{tabular}{ll} 
AWM Specification: & UL Style $1354\left(30 \mathrm{~V} 60^{\circ} \mathrm{C}\right)$ \\
\hline EU CE Mark: & Yes \\
\hline EU Directive 2000/53/EC (ELV): & Yes \\
\hline EU Directive 2002/95/EC (RoHS): & Yes \\
\hline EU RoHS Compliance Date (mm/dd/yyyy): & $01 / 01 / 2004$ \\
\hline EU Directive 2002/96/EC (WEEE): & Yes \\
\hline EU Directive 2003/11/EC (BFR): & Yes \\
\hline CA Prop 65 (CJ for Wire \& Cable): & Yes
\end{tabular}




\begin{tabular}{ll}
\hline MII Order \#39 (China RoHS): & Yes \\
\hline Military Specification: & MIL-C-17F \\
\hline RG Type: & $174 / \mathrm{U}$ \\
\hline
\end{tabular}

Plenum/Non-Plenum

$\operatorname{Plenum}(\mathbf{Y} / \mathbf{N})$ :

No

\section{Electrical Characteristics (Overall)}

Nom. Characteristic Impedance:

Impedance (Ohm)

50

Nom. Inductance:

Inductance $(\mu \mathrm{H} / \mathrm{m})$

0.252637

Nom. Capacitance Conductor to Shield:

Capacitance (pF/m)

101.055

Nominal Velocity of Propagation:

VP (\%)

66

Nominal Delay:

Delay (ns/m)

5.05274

Nom. Conductor DC Resistance:

DCR @ 20으 (Ohm/km)

318.257

Nominal Outer Shield DC Resistance:

DCR @ 20으 (Ohm/km)

35.1067

Nom. Attenuation:

Freq. (MHz) Attenuation (dB/100m)

$1 \quad 6.2339$

$10 \quad 10.8273$

$50 \quad 19.0298$

$100 \quad 27.5604$

$200 \quad 41.0125$

$400 \quad 62.339$

$700 \quad 88.587$

$900 \quad 101.711$

Max. Power Rating:

Freq. (MHz) Rating (W)

1976

$10 \quad 354$

$50 \quad 197$

$100 \quad 161$

$200 \quad 136$

$400 \quad 120$

$700 \quad 112$

$900 \quad 109$

Max. Operating Voltage - UL:

Voltage

30 V RMS (UL AWM Style 1354) 
Max. Operating Voltage - Non-UL:

Voltage

1100 V RMS (Military)

\section{Related Documents:}

No related documents are available for this product

\section{Put Ups and Colours:}

$\begin{array}{llllll}\text { Item \# } & \text { Put up } & \text { Ship Weight } & \text { Colour } & \text { Notes } & \text { Item Description } \\ 8216010100 & 30 \mathrm{MT} & 0.499 \mathrm{KG} & \text { BLACK } & & \text { \#26 PE BRD PVC } \\ 82160101000 & 305 \mathrm{MT} & 4.082 \mathrm{KG} & \text { BLACK } & \text { GN } & \text { \#26 PE BRD PVC } \\ & 152 \mathrm{MT} & 2.268 \mathrm{KG} & \text { BLACK } & & \end{array}$

\section{Notes:}

$\mathrm{G}=$ MAY CONTAIN MORE THAN 1 PIECE. MINIMUM LENGTH OF ANY ONE PIECE IS 100' $\mathrm{N}=$ FINAL PUT-UP LENGTH MAY VARY $-0 \%$ TO + $10 \%$ FROM LENGTH SHOWN. 


\section{$\underline{\text { Appendix B }}$}

\section{BNC Plug}

\section{Specification Table}

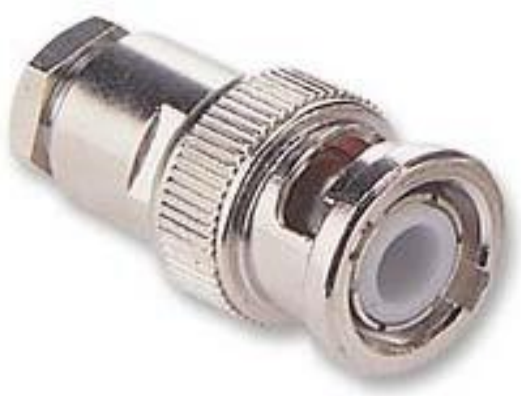

\section{Specifications:}
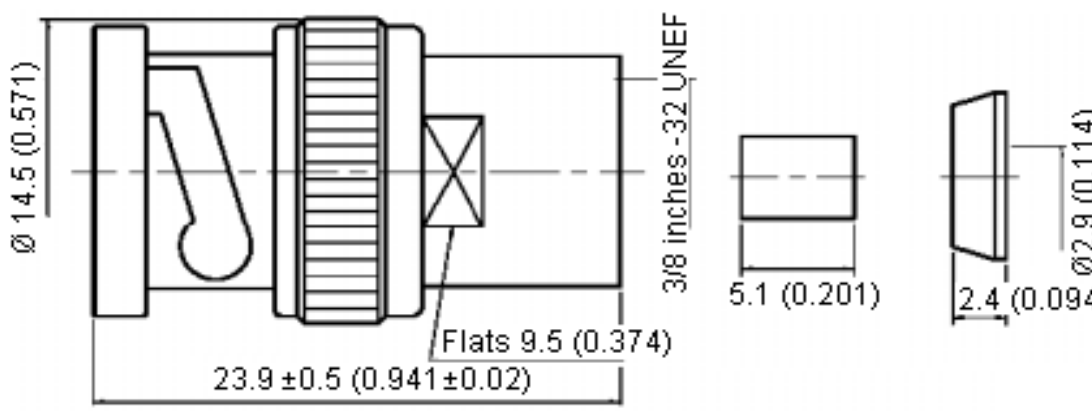

: Brass per JIS-C3604

Bodies

: Brass per JIS-C3604

Connector interface per MIL-STD-348A

Part to be clean and free of burrs
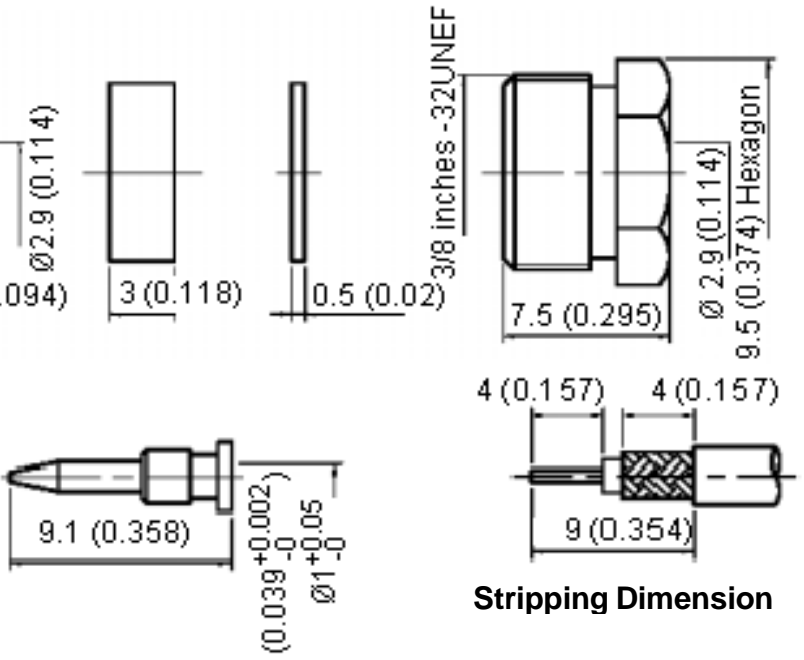

Stripping Dimension

Dimensions: Millimetres (Inches)

\begin{tabular}{|c|c|c|c|c|}
\hline Number & Description & Material & Finish & Part Number \\
\hline 1 & Shell & Zinc & \multirow{2}{*}{ Nickel $100 \mu$ Inches Minimum } & \multirow{13}{*}{$\begin{array}{c}\text { 13-01-3 TGZ } \\
\text { RG174U }\end{array}$} \\
\hline 2 & Body & Brass & & \\
\hline 3 & Insulation & PTFE & & \\
\hline 4 & Gasket & Rubber & None & \\
\hline 5 & Washer & Brass & Nickel $100 \mu$ Inches Minimum & \\
\hline 6 & Insulation & PTFE & None & \\
\hline 7 & Spring Washer & SK-5 & \multirow{3}{*}{ Nickel $100 \mu$ Inches Minimum } & \\
\hline 8 & Washer & \multirow{2}{*}{ Brass } & & \\
\hline 9 & Braid Clamp & & & \\
\hline 10 & Gasket & Rubber & None & \\
\hline 11 & Washer & \multirow{3}{*}{ Brass } & \multirow{2}{*}{ Nickel $100 \mu$ Inches Minimum } & \\
\hline 12 & Nut & & & \\
\hline 13 & Centre Contact & & Gold & \\
\hline
\end{tabular}

B. 1 


\section{APPENDIX C}

\section{$\underline{\text { Newton-Raphson Method }}$}

The Newton-Raphson method is an iterative procedure used to determine the root of an equation. In some simple situations, the root is easy to find. We consider Eqn. C1:

$f(x)=x-3$

Clearly the root of $f(x)$, the value such that $f(x)=0$, is when $x=3$.

A slightly more complicated example is a generic quadratic equation, Eqn. C2:

$f(x)=a x^{2}+b x+c$

Whose solution is in the form as given in Eqn. C3:

$x=\frac{-b \pm \sqrt{b^{2}-4 a c}}{2 a}$

However, what happens when we need to compute the root of an arbitrary function that does not have an analytical solution? For example, how would we find the root of the following function (eqn.C4):

$f(x)=x^{\frac{5}{6}}+e^{x^{2}}$

The answer, in this case, is not so easily determined. The simplest solution is to guess a possible range that might contain the answer and then step through each value until the answer is found. This brute-force method, however, is highly unreliable and extremely slow. A more robust and faster solution is to use the Newton-Raphson iterative method.

The method requires the analyst to start with a guess, $x_{O}$, and then the process iterates through successive values of $x$ until the root is found. The step size of the guess and the direction of the step are determined not only by the value at $\mathrm{f}(\mathrm{x})$ but also by its slope, or derivative. The derivation of the method is simple, but it provides deep insight into how the process works. To begin, we can write the definition of a derivative eqn. C5:

$\frac{d f\left(x_{n}\right)}{d x}=f^{\prime}\left(x_{n}\right)=\frac{f\left(x_{n}\right)-0}{x_{n}-x_{n+1}}$

The zero in the numerator is there because we are trying to arrive at the root, with a value of zero and with the hope that this happens at $x_{n+1}$. This equation can be rewritten to give the formal definition of the Newton-Raphson procedure eqn. C6:

$x_{n+1}=x_{n}-\frac{f\left(x_{n}\right)}{f^{\prime}\left(x_{n}\right)}$

The reason why this equation doesn't just "work" is that a true definition of a derivative is an instantaneous change in $x$. If $x_{n}$ is too far from the root, then the slope as computed by 
Eqn. C5 would not be a very accurate representation of the derivative. In other words, there is too much error in the representation, and an iterative process is required to converge onto the right answer. Figure C1 provides a graphical view of how the iteration works. At point $a$, the slope is positive but the value of the function is negative. Thus the next step in the iteration would result in an increase in $x$. At point $b$, the slope is still positive but the value is also positive, resulting in a decrease in $x$. Point $c$ is an illustration of how crucial the starting guess, $x_{0}$, is regarding the stability of the iteration. Notice at point $c$ that the slope is effectively zero and would cause the second term in eqn. C6 to effectively "blow up." When applying this method, care should be taken to not start off in this region.

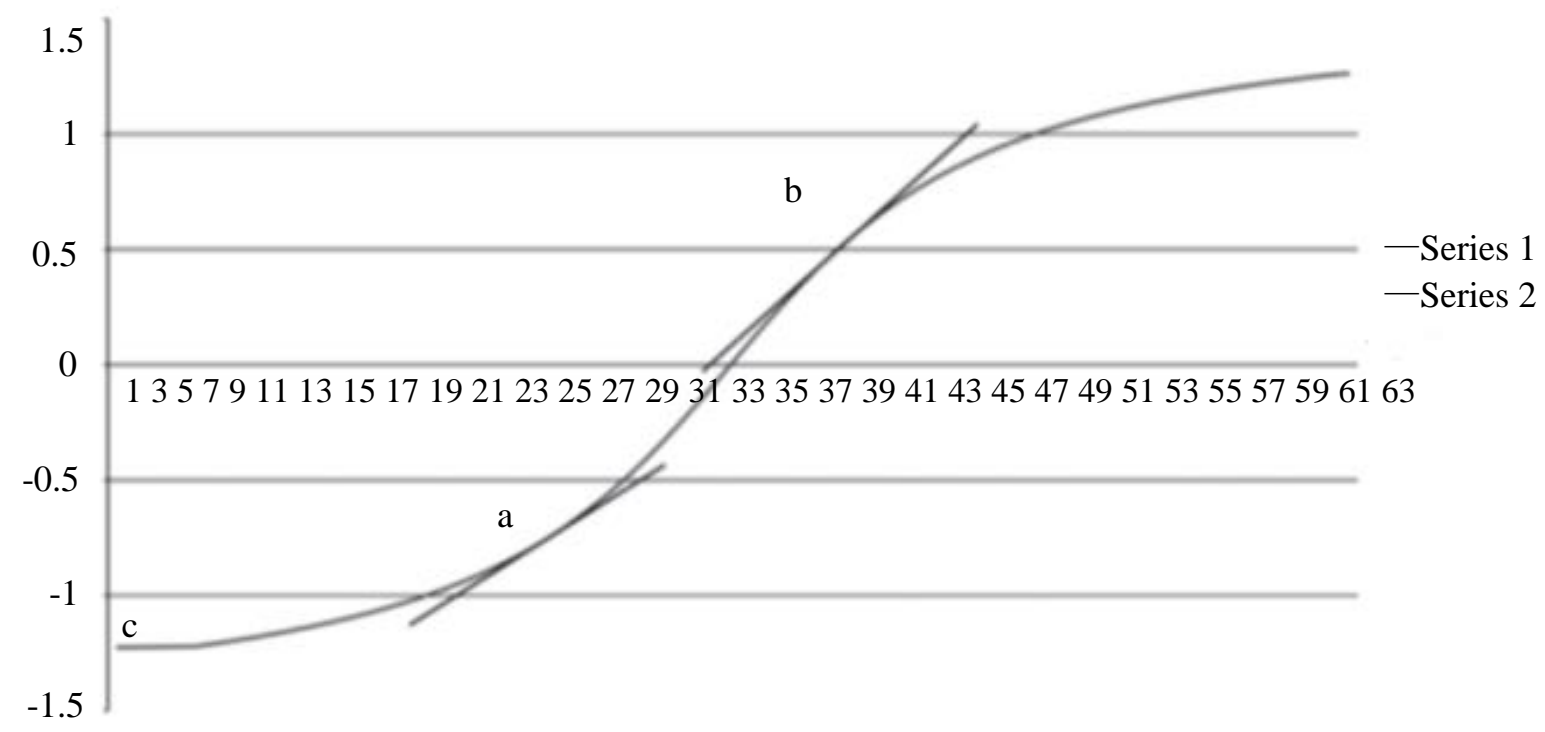

Figure C1 Point $\boldsymbol{a}$ would cause a jump forward, while point $\boldsymbol{b}$ would cause a jump backward. Point $\boldsymbol{c}$ is unstable because the slope is nearly 0 after Allman et al [5.5]. 


\section{APPENDIX D}

\section{Taylor Series Expansion}

\section{Function for one variable}

For a function of one variable, $f(x)$, the Taylor Series formula is:

$f(x+\Delta x)=f(x)+f^{\prime}(x) \Delta x+\frac{1}{2} f^{\|}(x)(\Delta x)^{2}+\ldots+\frac{1}{n !} f^{(n)}(x)(\Delta x)^{n}+\ldots$

Where $f^{\prime}(x)$ is the first derivative, $f^{\|}(x)$ the second derivative, $f^{(n)}(x)$ the $n t h$ derivative, and so on. In practice, usually the first two derivatives are used, and the effect of the remaining higher-order terms ignored:

$f(x+\Delta x)=f(x)+f^{\prime}(x) \Delta x+\frac{1}{2} f^{\prime \prime}(x)(\Delta x)^{2}+$ Higher Order Terms 


\section{APPENDIX E}

\section{Quadratic Equation}

A quadratic equation is one in which highest power of $x$ is 2 . The general form of a quadratic equation is given by:

$a x^{2}+b x+c=0$

Where $a, b$, and $c$ are numbers. For given values of $a, b$, and $c$, the values of $x$ that satisfy the equation are called solutions of the equation. Where $x$ can be calculated with the quadratic formula given by:

$x=\frac{-b \pm \sqrt{b^{2}-4 a c}}{2 a}$

The "plus or minus" sign $( \pm)$ indicates that there are always two possible values for $x$. In any real physical system, however, only one of the values will have any meaning. 